\title{
DESIGN AND DEVELOPMENT OF OPTICAL WAVEGUIDE ARCHITECTURES FOR REAL-TIME TEMPERATURE \\ MONITORING IN EXTREME ENVIRONMENTS
}

by

Bahareh Badamchi

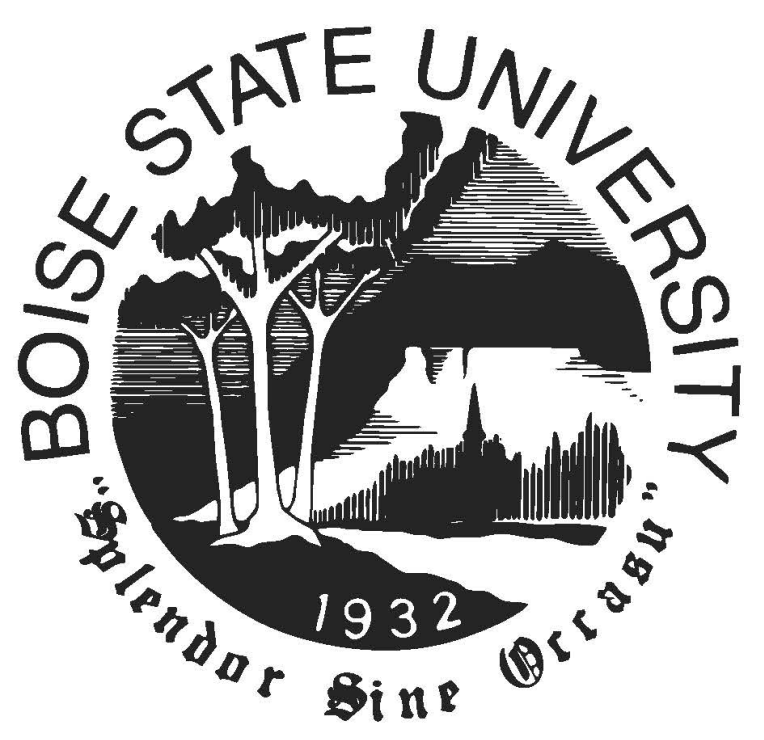

A dissertation

submitted in partial fulfillment

of the requirements for the degree of

Doctor of Philosophy in Electrical and Computer Engineering

Boise State University

May 2021 
(C) 2021

Bahareh Badamchi

ALL RIGHTS RESERVED 
BOISE STATE UNIVERSITY GRADUATE COLLEGE

\title{
DEFENSE COMMITTEE AND FINAL READING APPROVALS
}

of the dissertation submitted by

\author{
Bahareh Badamchi
}
Dissertation Title: Design and Development of Optical Waveguide Architectures for Real-Time Temperature Monitoring in Extreme Environ- ments

Date of Final Oral Examination: $\quad 01$ April 2021

The following individuals read and discussed the dissertation submitted by student Bahareh Badamchi, and they evaluated the student's presentation and response to questions during the final oral e xamination. They found that the student passed the final oral examination.

\author{
Harish Subbaraman, Ph.D. Chair, Supervisory Committee \\ Maria Mitkova, Ph.D. Member, Supervisory Committee \\ Nirmala Kandadai, Ph.D. Member, Supervisory Committee \\ Devinder Saini, Ph.D. Member, External Examiner
}

The final reading approval of the dissertation was granted by Harish Subbaraman, Ph.D., Chair of the Supervisory Committee. The dissertation was approved by the Graduate College. 


\section{DEDICATION}

This dissertation is dedicated to my lovely husband, parents, and martyr brother for their support and motivation over the years. 


\section{ACKNOWLEDGMENTS}

This work would not exist without the help of many people.

First and foremost, I wish to express my sincere appreciation to my advisor, Prof. Harish Subbaraman, who has the substance of generosity. He continuously supported and encouraged me to be professional and do the right thing even when the road got tough. I am thankful to him for giving me the freedom on choosing various, yet coherent research topics and directions and collaborators without objection. Prof. Subbaraman provided me with great opportunity to work with brilliant researchers during my Ph.D. work. I am also grateful for his valuable advice and great supervision that truly helped me improve my analysis, implementation, writing, and presentation skills which has made this project an unforgettable experience. Without his patience, motivation, enthusiasm, and immense knowledge, the goal of this project would not have been realized.

From the beginning days that I was working on my Ph.D. work, I have had the opportunity to closely work with Prof. Maria Mitkova. I would like to thank Prof. Mitkova for her priceless advice and efforts providing me with her insightful comments that substantially improved the quality of my research papers and this dissertation. Her experience and expertise are of great importance for our research. I am thankful to have her as my committee member.

Besides, I would like to thank the rest of my committee members, Prof. Nirmala 
Kandadai, for her encouragement, insightful comments, and questions. I always enjoyed my technical discussion with her during the research. I am grateful for Prof. Zeynep Hansen for being my graduate faculty representative. I am also grateful for Dr. Devinder Saini for being my external examiner.

I would like to extend my gratitude towards all the staff in the ECE Department especially the manager, Jennifer Ambrose, and the academic programs assistant, Baily Hazzard.

I would like to thank Prof. Alan Wang, Dr. Erwen Li, and Hsu Wei-Che from Oregon State University for their advice and efforts in providing me with their insightful comments that substantially improved the quality of my research. I would like to thank them for hosting me at Engineering Photonics Research Laboratory (EPRL), Oregon State University at Portland. During my visit at Oregon State University, I had many technical meetings and fruitful discussions and I am thankful for such an experience.

I am grateful to Dr. Pilar Herrera-Fierro and Dr. Vishva Ray from Lurie Nanofabrication Facility (LNF) at the University of Michigan for their valuable advice, help and comments on realization of my device.

I also appreciate the help from staff members from Idaho Microfabrication Laboratory (IML) and Boise State Center for Materials Characterization for their careful maintenance of the scientific tools. I would like to especially mention the assistance and advice from Pete Miranda, Travis Gabel and, Dr. Nick Bulloss for training me on a number of instruments on development of this research work and being patient with my questions.

I am also thankful to Al-Amin Ahmed Simon for his valuable contribution on 
my research. I would like to extend my gratitude to my lab mates at Boise State University: Ashita Chandnani, Sohel Rana, Anupama Dhamala, Jacob Manzi and Sarah Seva, for their friendship and the stimulating discussions.

I would like to thank my parents who encouraged me to pursue my dreams when I was young and help me out when I am in need. Without their longtime support in my study, I would not go this far to pursue this Ph.D. degree. I am thankful to my brothers and sister for supporting me at every step of my life.

Last but not the least, words cannot express how grateful I am to my lovely husband, Mojtaba. Without his unconditional support and encouragement, I could have never been able to finish this dissertation. 


\section{ABSTRACT}

This dissertation prompts on the research and development of a new real-time, reusable, and reversible optical sensor for integrated temperature monitoring in harsh environments. This is achieved through integrating the photonic properties of optical waveguides/optical fiber and the phase change properties of chalcogenide glasses (ChGs). ChG materials have very specific crystallization temperatures beyond which these materials transform from being a dielectric material to a metallic material. When such ChG material is coated over a dielectric optical waveguide, in the crystalline phase, highly localized surface plasmon polariton (SPP) modes are generated at the waveguide:metallic ChG interface. In this case, the modes are characterized by very large propagation losses compared to that when the $\mathrm{ChG}$ is in its amorphous phase. By monitoring the output power in the two different phases of $\mathrm{ChG}$, ambient temperature can be determined. In ChG materials, the crystalline state of ChGs can be reverted back to its initial amorphous condition through the application of a short intense voltage pulse that melts the material, facilitating multiple time use of the sensors. Based on the phase change property of ChG glasses, and light confinement offered by optical waveguides, we proposed to construct two types of sensor architectures for sensing temperature:

- Architecture 1: An optical fiber based reflection mode sensor.

- Architecture 2: An integrated silicon waveguide:ChG based compact plasmonic 
temperature sensor.

These sensors are typically suitable for the real-time monitoring of component temperatures up to $500{ }^{\circ} \mathrm{C}$, although with specific adjustment of the composition of the ChG material, these sensors can become useful for metallic or ceramic SFR reactors where the cladding temperature can reach $650{ }^{\circ} \mathrm{C}$. This will provide a temperature monitoring method for multiple components in the reactor design domain of multiple reactors. It can be further employed as in a number of hybrid electron/photonic tandem $\mathrm{ChG} / \mathrm{Si}$ solutions (for example, when non-volatile memory is necessary to be introduced based also on the phase changes in the $\mathrm{ChG}$ ) in the nuclear facilities since the chalcogenide glasses are radiation hard materials. 


\section{TABLE OF CONTENTS}

ACKNOWLEDGMENT . . . . . . . . . . . . . . . . . v v

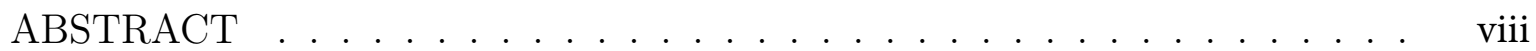

LIST OF FIGURES . . . . . . . . . . . . . . . . . . xiv

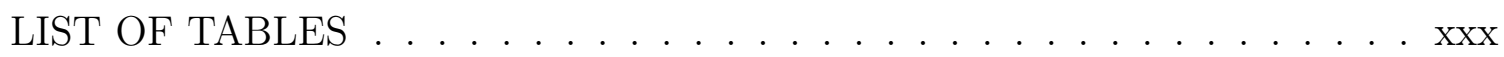

LIST OF ABBREVIATIONS . . . . . . . . . . . . . . . . .xxxii

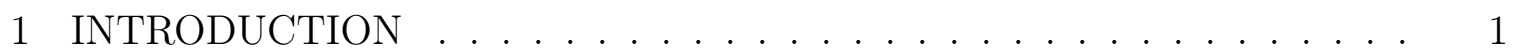

1.1 Traditional Electronic Sensors Used in Extreme Environments . . . . 1

1.2 Summary of Contributions . . . . . . . . . . . . . . 5

1.3 List of Publications . . . . . . . . . . . . . . . . 7

1.3.1 Journal Papers . . . . . . . . . . . . . . . . . 7

1.3.2 Conference Papers . . . . . . . . . . . . . . . 7

1.3 .3 Showcase ................... 8

1.3.4 Patent ....................... 8

1.4 List of Awards . . . . . . . . . . . . . . . . . . . . 9

1.5 Dissertation Outline . . . . . . . . . . . . . . . 9 


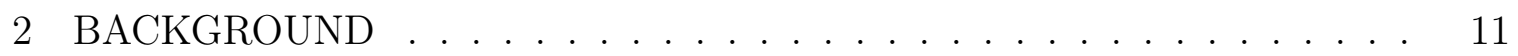

2.1 Optical Waveguides . . . . . . . . . . . . . . . . . . 11

2.1 .1 Optical Fiber . . . . . . . . . . . . . . . . . . 11

2.1.2 Planar Silicon Optical Waveguide . . . . . . . . . . . . . 14

2.1.3 Surface Plasmon Waveguiding _. . . . . . . . . . 20

2.1.4 Coupling to Waveguide . . . . . . . . . . . . . . . . 28

2.1.5 Chalcogenide Glasses . . . . . . . . . . . . . . . . . . . 34

3 OPTICAL FIBER BASED TEMPERATURE SENSOR MODELING . . 38

3.1 Determination of the Optical Constants of In-house Synthesized ChGs 38 3.1 .1 Fiber Sensor Modeling . . . . . . . . . . . . . . . . 42

3.2 Summary . . . . . . . . . . . . . . . . . . 52

4 FABRICATION AND CHARACTERIZATION OF OPTICAL FIBER BASED TEMPERATURE SENSORS . . . . . . . . . . . . . . 54

4.1 Optical Fiber Tip Coating . . . . . . . . . . . . . . . . . 54

4.1 .1 Dip Coating . . . . . . . . . . . . . . . 56

4.1 .2 Thermal Evaporation . . . . . . . . . . . . . . 58

4.2 Experimental Setup . . . . . . . . . . . . . . . . . . . 60

4.3 Results and Discussion . . . . . . . . . . . . . . . . 61

4.4 Temperature Profile Estimation Using Array Sensor $\ldots \ldots$. . . . . 66

4.5 Summary . . . . . . . . . . . . . . . . . . 69

5 MODELING AN INTEGRATED SILICON WAVEGUIDE:CHG BASED COMPACT PLASMONIC TEMPERATURE SENSOR . . . . . . . . . 71

5.1 Optical Waveguide Structure . . . . . . . . . . . . . . . . 71 
5.1.1 Design 1 (ChG covering silicon waveguide) . . . . . . . . . 74

5.1.2 Design 2 (A thin ChG layer sandwiched between Si waveguide and metal) . . . . . . . . . . . . . . . . . . 79

5.1.3 Design 3 (ChG glass patterned on the top surface of silicon waveguide) . . . . . . . . . . . . . .

5.1.4 Design 4 (ChG misaligned and covering surface and one side of silicon waveguide) . . . . . . . . . . . . . . . 86

5.2 Design of Plasmonic based Temperature Sensor Using Characterization

Data from In-House Synthesized Inks . . . . . . . . . . . . . . . . . 91

5.2.1 Design 1 (in-house synthesized inks covering Silicon Waveguide) 91

5.2.2 Design 3 (in-house synthesized inks on the top surface of Silicon

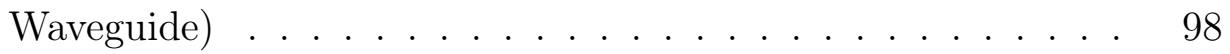

5.2.3 Design 4 (in-house synthesized inks misaligned and covering surface and one side of silicon waveguide) . . . . . . . . . 104

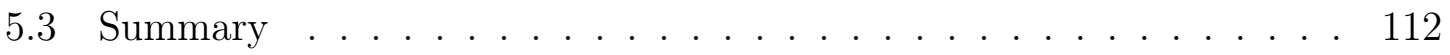

6 FABRICATION OF SILICON:CHG WAVEGUIDE BASED PLASMONIC TEMPERATURE SENSORS . . . . . . . . . . . . . . . . . 114

6.1 Fabrication Procedure . . . . . . . . . . . . . . . . . . . 114

6.2 Waveguide Grating Coupler Fabrication . . . . . . . . . . . . . 117

6.2.1 Dose test on grating coupler section . . . . . . . . . . . . . . . 117

6.2.2 Stitching . . . . . . . . . . . . . . . 123

6.2.3 Grating coupler terminated silicon waveguide fabrication using registration marks . . . . . . . . . . . . . . 133

6.3 S-bend Waveguide Fabrication . . . . . . . . . . . . . . . . . . 134 
6.4 In-house Synthesized Ink Covering of the Silicon Waveguide $\ldots \ldots .136$

6.5 Experimental Setup . . . . . . . . . . . . . . . . . . . 137

6.6 Results and Discussion . . . . . . . . . . . . . . . . . . . . . 141

6.7 Summary . . . . . . . . . . . . . . . . . . 144

7 FUTURE RESEARCH $\ldots \ldots \ldots \ldots \ldots$

7.1 Packaging Details for Waveguide based Temperature Sensors . . . . 146

7.2 Integration of Arrays Si waveguide:ChG based Temperature Sensor . 149

7.3 Sensitivity Analysis of Si waveguide:ChG based Temperature Sensor . 151

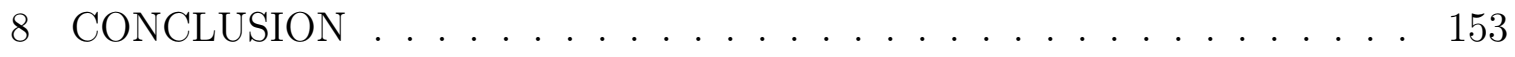

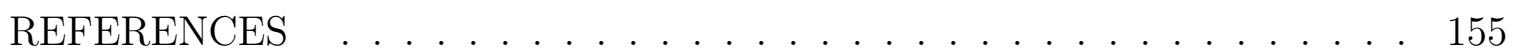

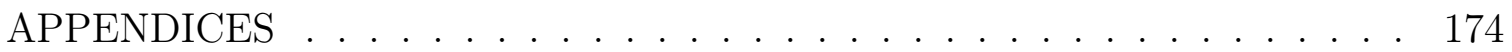

A LIMITATIONS OF OUR EBL TOOL-MODIFICATIONS PERFORMED TO IMPROVE PERFORMANCE . . . . . . . . . . . . . . . 175

A.1 Beam blanker leakage . . . . . . . . . . . . . 176

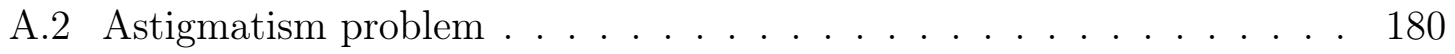

A.3 Low beam current . . . . . . . . . . . . . . . . . . . . . . . . 181

B FABRICATION PROCESS DETAILS . . . . . . . . . . . . . 183 


\section{LIST OF FIGURES}

2.1 Schematic of optical fiber, showing typical diameter [33]. . . . . . . . 12

2.2 Propagation of light inside the fiber based on total internal reflection. 13

2.3 Planar waveguide of one-dimensional transverse optical confinement [34]. 14

2.4 Index profiles of (a) a step-index planar waveguide and (b) a gradedindex planar waveguide [34]. . . . . . . . . . . . . . . . . . 15

2.5 Light rays and their phase fronts in the waveguide [46]. . . . . . . . 16

2.6 Possible modes can be classified in terms of (a) transverse electric field (TE), and (b) transverse magnetic field (TM) . . . . . . . . . . 18

2.7 Channel waveguides, a) Buried channel waveguide, b) Strip-loaded waveguide, c) Ridge waveguide, d) Diffused waveguide, e) Rib waveguide. 19

2.8 A schematic of the electromagnetic wave and surface charges at a metal-dielectric interface with semi-infinitely extended media on either side [58]. . . . . . . . . . . . . . . . . . . . . . . . . . . . . 23

2.9 Field profile at a metal-dielectric interface [58]. . . . . . . . . . 25

2.10 Mode mismatch between Si waveguide and optical fiber. . . . . . . . 28

2.11 Schematic of a) butt coupling, b) end-fire coupling, c) prism coupling, d) grating coupling. . . . . . . . . . . . . . . . . . . . 29

2.12 Schematic of a) an inverse taper [67], b) edge coupler using inverse-tapers. 32

2.13 Coupling light through grating coupler to an SOI waveguide [69]. . . 33 
3.1 Illustration of the setup of an ellipsometry measurement [92] . . . . .

3.2 Schematic cross-section of proposed ChG-capped optical fiber tip-based temperature sensor. . . . . . . . . . . . . . . .

3.3 Schematic cross-section of proposed ChG-capped optical fiber tip-based temperature sensor. . . . . . . . . . . . . . . . . .

3.4 fiber-optic sensor based on Fresnel reflection. . . . . . . . . . . . . .

3.5 $\mathrm{Ge}_{40} \mathrm{Se}_{60}$ capped fiber device: Power distribution at the fiber ChG interface for: a) transmitted power in amorphous phase, b) transmitted power in crystalline phase, c) transmitted power as a function of the length of $\mathrm{ChG}, \mathrm{d})$ reflected power as a function of the length of ChG. Solid curve indicates crystalline phase and the dashed-dotted curve indicates amorphous phase. . . . . . . . . . . . . .

3.6 $\mathrm{Ge}_{30} \mathrm{Se}_{70}$ capped fiber device: Power distribution at the fiber ChG interface for: a) transmitted power in amorphous phase, b) transmitted power in crystalline phase, c) transmitted power as a function of the length of $\mathrm{ChG}$, d) reflected power as a function of the length of ChG. Solid curve indicates crystalline phase and the dashed-dotted curve indicates amorphous phase. . . . . . . . . . . . . . .

3.7 $\mathrm{Ge}_{33} \mathrm{Se}_{67}$ capped fiber device: Power distribution at the fiber $\mathrm{ChG}$ interface for: a) transmitted power in amorphous phase, b) transmitted power in crystalline phase, c) transmitted power as a function of the length of $\mathrm{ChG}$, d) reflected power as a function of the length of ChG. Solid curve indicates crystalline phase and the dashed-dotted curve indicates amorphous phase. . . . . . . . . . . . . . 
3.8 $\mathrm{Ge}_{40} \mathrm{~S}_{60}$ capped fiber device: Power distribution at the fiber $\mathrm{ChG}$ interface for: a) transmitted power in amorphous phase, b) transmitted power in crystalline phase, c) transmitted power as a function of the length of $\mathrm{ChG}$, d) reflected power as a function of the length of ChG. Solid curve indicates crystalline phase and the dashed-dotted curve indicates amorphous phase. . . . . . . . . . . . .

$3.9 \mathrm{Ge}_{30} \mathrm{~S}_{70}$ capped fiber device: Power distribution at the fiber ChG interface for: a) transmitted power in amorphous phase, b) transmitted power in crystalline phase, c) transmitted power as a function of the length of $\mathrm{ChG}$, d) reflected power as a function of the length of ChG. Solid curve indicates crystalline phase and the dashed-dotted curve indicates amorphous phase. . . . . . . . . . . . . .

$3.10 \mathrm{Ge}_{33} \mathrm{~S}_{67}$ capped fiber device: Power distribution at the fiber ChG interface for: a) transmitted power in amorphous phase, b) transmitted power in crystalline phase, c) transmitted power as a function of the length of $\mathrm{ChG}, \mathrm{d})$ reflected power as a function of the length of ChG. Solid curve indicates crystalline phase and the dashed-dotted curve indicates amorphous phase. . . . . . . . . . . . . . .

3.11 a) Normalized reflected power of the fibers capped with in-house synthesized Ge-S (top row) and Ge-Se (bottom row) compositions. Solid curve indicates crystalline phase and the dashed-dotted curve indicates amorphous phase, b) Normalized reflected power of Ge-Se and Ge-S coated fiber tips in amorphous and crystalline phases. . . . . . . .

4.1 Gold coated fiber [100]. . . . . . . . . . . . . . . . 
4.2 Aqua Regia solution, b) gold coated fiber is dipped in solution. . . . . 56

4.3 a) Gold coated fiber under microscope, b) etching after 600 seconds,

c) Transition part between etch and gold. . . . . . . . . . . . . . . 56

4.4 Fiber coating method by immersion of the fiber into ink or melt of ChG. 57

4.5 Dip-coating fabrication method. a) experiment setup to coat fiber tip with chalcogenide glass, b) an ink coated fiber tip (dark) and a blank fiber tip (transparent). . . . . . . . . . . . . . . . . . 58

4.6 Thermal Evaporation fabrication method, a) Experiment setup to coat fiber tip with chalcogenide glass, b) chalcogenide glass covered fiber-tip. 60

4.7 (a) Schematic of setup for testing the temperature performance of the fabricated sensors, (b) a picture of the actual device characterization

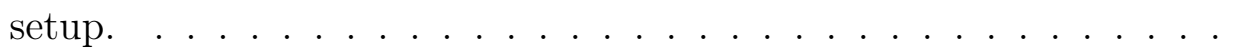

4.8 Simulated and measured normalized reflected power as a function of time with $\mathrm{Ge}_{30} \mathrm{~S}_{70}$ capped fiber tip. . . . . . . . . . . . . . . 62

4.9 Temperature response of evaporated $\mathrm{Ge}_{40} \mathrm{Se}_{60}$ capped fiber-tip based temperature sensor. . . . . . . . . . . . . . . .

4.10 Simulated and measured normalized reflected power as a function of time with $\mathrm{Ge}_{40} \mathrm{Se}_{60}$ capped fiber tip. . . . . . . . . . . . .

4.11 a) Optical fiber array with different ChG-capped fiber optics, b) Reflected output power in array structure, c) Temperature versus time. . 67

4.12 a) Temperature response of the sensor array, b) Monitored temperature trend as a function of time using array structure. . . . . . . . . . . . 68

5.1 a) Cross section of Design 1, b) 3D view of Design 1. . . . . . . . . 74 
5.2 Amorphous Phase, a) Intensity profile of TE mode, b) Intensity distribution along the waveguide, and c) Output power of TE mode as a function of the length of the waveguide. . . . . . . . . . . . 76

5.3 Amorphous phase, a) Intensity profile of TM mode, b) Intensity distribution along the waveguide, and c) Output power of TM mode as a function of the length of the waveguide. . . . . . . . . . . . 76

5.4 Crystalline Phase, a) Intensity profile of TE mode, b) Intensity distribution along the waveguide, and c) Output power of TE mode as a function of the length of the waveguide. . . . . . . . . . . . . 78

5.5 Crystalline Phase, a) Intensity profile of TM mode, b) Intensity distribution along the waveguide, and c) Output power of TM mode as a function of the length of the waveguide. . . . . . . . . . . 78

5.6 Extinction ratio of Design 1 in TM mode. . . . . . . . . . . . . . 79

5.7 a) Cross section of Design 2, b) 3D view of Design 2. . . . . . . . . 80

5.8 Amorphous phase a) Intensity profile of TE mode, b) Intensity distribution along the waveguide, and c) Output power of TE mode as a function of the length of the waveguide. . . . . . . . . . .

5.9 Amorphous phase a) Intensity profile of TM mode, b) Intensity distribution along the waveguide, and c) Output power of TM mode as a function of the length of the waveguide. . . . . . . . . .

5.10 Crystalline phase a) Intensity profile of TE mode, b) Intensity distribution along the waveguide, and c) Output power of TE mode as a function of the length of the waveguide. . . . . . . . . . . . 
5.11 Crystalline phase a) Intensity profile of TM mode, b) Intensity distribution along the waveguide, and c) Output power of TM mode as a function of the length of the waveguide. . . . . . . . . . . 83

5.12 a) Cross section of Design 3, b) 3D view of Design 3. . . . . . . . . . 84

5.13 Amorphous phase a) Intensity profile of TM mode, b) Intensity distribution along the waveguide, and c) Output power of TM mode as a function of the length of the waveguide. . . . . . . . . . . .

5.14 Crystalline phase a) Intensity profile of TM mode, b) Intensity distribution along the waveguide, and c) Output power of TM mode as a function of the length of the waveguide. . . . . . . . . . . . . 86

5.15 a) Cross section of design 4 , b) 3D view of design $4 \ldots \ldots$. . . . . 87

5.16 Amorphous phase a) Intensity profile of TE mode, b) Intensity distribution along the waveguide, and c) Output power of TE mode as a function of the length of the waveguide. . . . . . . . . . 88

5.17 Amorphous phase a) Intensity profile of TM mode, b) Intensity distribution along the waveguide, and c) Output power of TM mode as a function of the length of the waveguide. . . . . . . . . . .

5.18 Crystalline phase a) Intensity profile of TE mode, b) Intensity distribution along the waveguide, and c) Output power of TE mode as a function of the length of the waveguide. . . . . . . . . . . . .

5.19 Crystalline phase a) Intensity profile of TM mode, b) Intensity distribution along the waveguide, and c) Output power of TM mode as a function of the length of the waveguide. . . . . . . . . . . . 
5.20 Amorphous $\mathrm{Ge}_{30} \mathrm{~S}_{70}$ patterned on the top surface of silicon waveguide in crystalline phase. a) Intensity profile of TM mode, b) Intensity distribution along the waveguide, and c) Output power of TM mode as a function of the length of the waveguide. . . . . . . . . . .

5.21 Crystalline $\mathrm{Ge}_{30} \mathrm{~S}_{70}$ patterned on the top surface of silicon waveguide in crystalline phase. a) Intensity profile of TM mode, b) Intensity distribution along the waveguide, and c) Output power of TM mode as a function of the length of the waveguide. . . . . . . . . . .

5.22 Amorphous $\mathrm{Ge}_{40} \mathrm{~S}_{60}$ patterned on the top surface of silicon waveguide in crystalline phase. a) Intensity profile of TM mode, b) Intensity distribution along the waveguide, and c) Output power of TM mode as a function of the length of the waveguide. . . . . . . . . . .

5.23 Crystalline $\mathrm{Ge}_{40} \mathrm{~S}_{60}$ patterned on the top surface of silicon waveguide in crystalline phase. a) Intensity profile of TM mode, b) Intensity distribution along the waveguide, and c) Output power of TM mode as a function of the length of the waveguide. . . . . . . . . . .

5.24 Amorphous $\mathrm{Ge}_{33} \mathrm{~S}_{67}$ patterned on the top surface of silicon waveguide in crystalline phase. a) Intensity profile of TM mode, b) Intensity distribution along the waveguide, and c) Output power of TM mode as a function of the length of the waveguide. . . . . . . . . . .

5.25 Crystalline $\mathrm{Ge}_{33} \mathrm{~S}_{67}$ patterned on the top surface of silicon waveguide in crystalline phase. a) Intensity profile of TM mode, b) Intensity distribution along the waveguide, and c) Output power of TM mode as a function of the length of the waveguide. . . . . . . . . . . 
5.26 Amorphous $\mathrm{Ge}_{30} \mathrm{Se}_{70}$ patterned on the top surface of silicon waveguide in crystalline phase. a) Intensity profile of TM mode, b) Intensity distribution along the waveguide, and c) Output power of TM mode as a function of the length of the waveguide. . . . . . . . . . . .

5.27 Crystalline $\mathrm{Ge}_{30} \mathrm{Se}_{70}$ patterned on the top surface of silicon waveguide in crystalline phase. a) Intensity profile of TM mode, b) Intensity distribution along the waveguide, and c) Output power of TM mode as a function of the length of the waveguide. . . . . . . . . . .

5.28 Amorphous $\mathrm{Ge}_{40} \mathrm{Se}_{60}$ patterned on the top surface of silicon waveguide in crystalline phase. a) Intensity profile of TM mode, b) Intensity distribution along the waveguide, and c) Output power of TM mode as a function of the length of the waveguide. . . . . . . . . . .

5.29 Crystalline $\mathrm{Ge}_{40} \mathrm{Se}_{60}$ patterned on the top surface of silicon waveguide in crystalline phase. a) Intensity profile of TM mode, b) Intensity distribution along the waveguide, and c) Output power of TM mode as a function of the length of the waveguide. . . . . . . . . .

5.30 Amorphous $\mathrm{Ge}_{33} \mathrm{Se}_{67}$ patterned on the top surface of silicon waveguide in crystalline phase. a) Intensity profile of TM mode, b) Intensity distribution along the waveguide, and c) Output power of TM mode as a function of the length of the waveguide. . . . . . . . . .

5.31 Crystalline $\mathrm{Ge}_{33} \mathrm{Se}_{67}$ patterned on the top surface of silicon waveguide in crystalline phase. a) Intensity profile of TM mode, b) Intensity distribution along the waveguide, and c) Output power of TM mode as a function of the length of the waveguide. . . . . . . . . . . 
5.32 Amorphous $\mathrm{Ge}_{30} \mathrm{~S}_{70}$ patterned on the top surface of silicon waveguide in amorphous phase. a) Intensity profile of TM mode, b) Intensity distribution along the waveguide, and c) Output power of TM mode as a function of the length of the waveguide. . . . . . . . . . .

5.33 Crystalline $\mathrm{Ge}_{30} \mathrm{~S}_{70}$ patterned on the top surface of silicon waveguide in crystalline phase. a) Intensity profile of TM mode, b) Intensity distribution along the waveguide, and c) Output power of TM mode as a function of the length of the waveguide. . . . . . . . . . .

5.34 Amorphous $\mathrm{Ge}_{40} \mathrm{~S}_{60}$ patterned on the top surface of silicon waveguide in amorphous phase. a) Intensity profile of TM mode, b) Intensity distribution along the waveguide, and c) Output power of TM mode as a function of the length of the waveguide. . . . . . . . . . .

5.35 Crystalline $\mathrm{Ge}_{40} \mathrm{~S}_{60}$ patterned on the top surface of silicon waveguide in crystalline phase. a) Intensity profile of TM mode, b) Intensity distribution along the waveguide, and c) Output power of TM mode as a function of the length of the waveguide. . . . . . . . . . . . . . 100

5.36 Amorphous $\mathrm{Ge}_{33} \mathrm{~S}_{67}$ patterned on the top surface of silicon waveguide in amorphous phase. a) Intensity profile of TM mode, b) Intensity distribution along the waveguide, and c) Output power of TM mode as a function of the length of the waveguide. . . . . . . . . . . . . 101

5.37 Crystalline $\mathrm{Ge}_{33} \mathrm{~S}_{67}$ patterned on the top surface of silicon waveguide in crystalline phase. a) Intensity profile of TM mode, b) Intensity distribution along the waveguide, and c) Output power of TM mode as a function of the length of the waveguide. . . . . . . . . . . . . . 101 
5.38 Amorphous $\mathrm{Ge}_{30} \mathrm{Se}_{70}$ patterned on the top surface of silicon waveguide in amorphous phase. a) Intensity profile of TM mode, b) Intensity distribution along the waveguide, and c) Output power of TM mode as a function of the length of the waveguide. . . . . . . . . . . 102

5.39 Crystalline $\mathrm{Ge}_{30} \mathrm{Se}_{70}$ patterned on the top surface of silicon waveguide in crystalline phase . a) Intensity profile of TM mode, b) Intensity distribution along the waveguide, and c) Output power of TM mode as a function of the length of the waveguide. . . . . . . . . . . . . 102

5.40 Amorphous $\mathrm{Ge}_{40} \mathrm{Se}_{60}$ patterned on the top surface of silicon waveguide in amorphous phase . a) Intensity profile of TM mode, b) Intensity distribution along the waveguide, and c) Output power of TM mode as a function of the length of the waveguide. . . . . . . . . . . . 103

5.41 Crystalline $\mathrm{Ge}_{40} \mathrm{Se}_{60}$ patterned on the top surface of silicon waveguide in crystalline phase. a) Intensity profile of TM mode, b) Intensity distribution along the waveguide, and c) Output power of TM mode as a function of the length of the waveguide. . . . . . . . . . . . . 103

5.42 Amorphous $\mathrm{Ge}_{33} \mathrm{Se}_{67}$ patterned on the top surface of silicon waveguide in amorphous phase. a) Intensity profile of TM mode, b) Intensity distribution along the waveguide, and c) Output power of TM mode as a function of the length of the waveguide. . . . . . . . . . . . . . . 104

5.43 Crystalline $\mathrm{Ge}_{33} \mathrm{Se}_{67}$ patterned on the top surface of silicon waveguide in crystalline phase. a) Intensity profile of TM mode, b) Intensity distribution along the waveguide, and c) Output power of TM mode as a function of the length of the waveguide. . . . . . . . . . . . . . 104 
5.44 Amorphous $\mathrm{Ge}_{30} \mathrm{~S}_{70}$ patterned on the top surface of silicon waveguide in crystalline phase. a) Intensity profile of TM mode, b) Intensity distribution along the waveguide, and c) Output power of TM mode as a function of the length of the waveguide. . . . . . . . . . . . 105

5.45 Crystalline $\mathrm{Ge}_{30} \mathrm{~S}_{70}$ patterned on the top surface of silicon waveguide in crystalline phase. a) Intensity profile of TM mode, b) Intensity distribution along the waveguide, and c) Output power of TM mode as a function of the length of the waveguide. . . . . . . . . . . 106

5.46 Amorphous $\mathrm{Ge}_{40} \mathrm{~S}_{60}$ patterned on the top surface of silicon waveguide in crystalline phase. a) Intensity profile of TM mode, b) Intensity distribution along the waveguide, and c) Output power of TM mode as a function of the length of the waveguide. . . . . . . . . . . . . 106

5.47 Crystalline $\mathrm{Ge}_{40} \mathrm{~S}_{60}$ patterned on the top surface of silicon waveguide in crystalline phase. a) Intensity profile of TM mode, b) Intensity distribution along the waveguide, and c) Output power of TM mode as a function of the length of the waveguide. . . . . . . . . . . . . . . 107

5.48 Amorphous $\mathrm{Ge}_{33} \mathrm{~S}_{67}$ patterned on the top surface of silicon waveguide in crystalline phase. a) Intensity profile of TM mode, b) Intensity distribution along the waveguide, and c) Output power of TM mode as a function of the length of the waveguide. . . . . . . . . . . . . 107

5.49 Crystalline $\mathrm{Ge}_{33} \mathrm{~S}_{67}$ patterned on the top surface of silicon waveguide in crystalline phase. a) Intensity profile of TM mode, b) Intensity distribution along the waveguide, and c) Output power of TM mode as a function of the length of the waveguide. . . . . . . . . . . . . 108 
5.50 Amorphous $\mathrm{Ge}_{30} \mathrm{Se}_{70}$ patterned on the top surface of silicon waveguide in crystalline phase. a) Intensity profile of TM mode, b) Intensity distribution along the waveguide, and c) Output power of TM mode as a function of the length of the waveguide. . . . . . . . . . . . 108

5.51 Crystalline $\mathrm{Ge}_{30} \mathrm{Se}_{70}$ patterned on the top surface of silicon waveguide in crystalline phase. a) Intensity profile of TM mode, b) Intensity distribution along the waveguide, and c) Output power of TM mode as a function of the length of the waveguide. . . . . . . . . . . . 109

5.52 Amorphous $\mathrm{Ge}_{40} \mathrm{Se}_{60}$ patterned on the top surface of silicon waveguide in crystalline phase. a) Intensity profile of TM mode, b) Intensity distribution along the waveguide, and c) Output power of TM mode as a function of the length of the waveguide. . . . . . . . . .

5.53 Crystalline $\mathrm{Ge}_{40} \mathrm{Se}_{60}$ patterned on the top surface of silicon waveguide in crystalline phase. a) Intensity profile of TM mode, b) Intensity distribution along the waveguide, and c) Output power of TM mode as a function of the length of the waveguide. . . . . . . . . . . . . . 110

5.54 Amorphous $\mathrm{Ge}_{33} \mathrm{Se}_{67}$ patterned on the top surface of silicon waveguide in crystalline phase. a) Intensity profile of TM mode, b) Intensity distribution along the waveguide, and c) Output power of TM mode as a function of the length of the waveguide. . . . . . . . . . . . . . . 110

5.55 Crystalline $\mathrm{Ge}_{33} \mathrm{Se}_{67}$ patterned on the top surface of silicon waveguide in crystalline phase. a) Intensity profile of TM mode, b) Intensity distribution along the waveguide, and c) Output power of TM mode as a function of the length of the waveguide. . . . . . . . . . . . . 111 
6.1 Process flow for fabricating Silicon:ChG waveguide based plasmonic temperature sensors . . . . . . . . . . . . . . . . 115

6.2 Proposed waveguide grating coupler design, a) Top view, b) 3D view. A waveguide is connected to input/output grating coupler using two taper waveguides. Waveguide width $=0.45 \mu \mathrm{m}$, waveguide length $=$ $150 \mu \mathrm{m}$, taper length $=250 \mu \mathrm{m}$, grating coupler length $=17.125 \mu \mathrm{m}$,

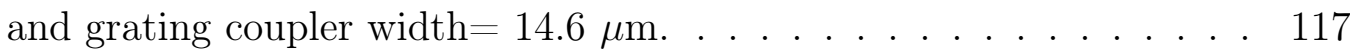

6.3 Grating coupler, a) top view, b) side view. $\mathrm{P}_{v}$ is vertical grating period, $\mathrm{P}_{h}$ is horizontal grating period, $\mathrm{L}$ is length of grating pitch, and $\mathrm{W}$ is width of grating pitch. Device layer (Si) thickness is $220 \mathrm{~nm}$. . . . . . 118

6.4 Grating couple design in DesignCAD with different dose. Each color shows exposure beam dose in Run File Editor in NPGs software. . . . 119

6.5 Low line dose effect test for grating coupler section of the design , a) $0.9 \mathrm{nC} / \mathrm{cm}, \mathrm{b}) 1.2 \mathrm{nC} / \mathrm{cm}, \mathrm{c}) 1.4 \mathrm{nC} / \mathrm{cm} \ldots \ldots . \ldots . . \ldots 121$

6.6 High line dose effect test for grating coupler section of the design. Dark areas are exposed by EBL. . . . . . . . . . . . . . . . . . . . . . 122

6.7 Line dose study on taper part of the design. . . . . . . . . . . . . . 122

6.8 Line dose study on waveguide part of the design. . . . . . . . . . . . . 123

6.9 Fracture with $80 \mu \mathrm{m}$ sub-fields. . . . . . . . . . . . . . . . . 125

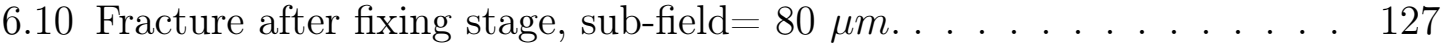

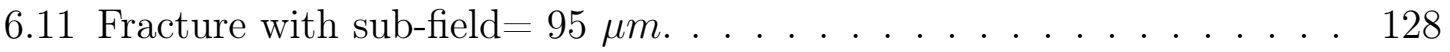

6.12 Blind stitching error, a) discontinuous, b) misalignment . . . . . . . . 129 
6.13 CAD image of photo mask with registration marks, a) photo-mask layout, b) Registration marks on one chip, c) global mark, d) chip mark, e) inset of marks in one array waveguides. . . . . . . . . . . . . 130

6.14 a) Fracture writing field to $200 \mu m \times 200 \mu m$ sub-fields, b) CAD design of array waveguides, c) Coarse and fine registration chip marks . . . .

6.15 Microscope image of fabricated array of subwavelength grating coupler ended waveguide, a) array of waveguide structure, b) waveguide, c) grating coupler. . . . . . . . . . . . . . . . . . . 134

6.16 CAD design of array S-bend waveguides . . . . . . . . . . . 135

6.17 SEM image of fabricated an array of S-bend waveguides. . . . . . . 136

6.18 A shadow mask fabricated at Boise State University on Kapton substrate for direct chalcogenide glass evaporation over the waveguides. . 137

6.19 Chalcogenide glass covered Si waveguide. . . . . . . . . . . . . . 137

6.20 a) Waveguide characterization set up at Boise State University, b) grating coupler testing setup schematic, c) close view of grating coupler testing setup, c) edge coupling testing set up schematic, d) close view of grating coupling testing set up. . . . . . . . . . . . . . 138

6.21 a) Aligning the fiber with the input grating coupler using red light, b) aligning the fiber with the output grating coupler using red light, c) temperature sensor device under temperature test. . . . . . . . . . . . 139

6.22 Testing set-up for characterizing the temperature response of grating terminated silicon waveguide based temperature sensor. . . . . . . . . 140

6.23 Measured transmission spectrum from the fabricated device without ChG cover layer on the waveguide. . . . . . . . . . . . . . . . . 141 
6.24 Temperature of heat stage as a function of time. . . . . . . . . . . . . 142

6.25 Simulated and measured normalized transmitted power as a function of time with $\mathrm{Ge}_{40} \mathrm{~S}_{60}$ covered silicon waveguide based temperature sensor. 143

6.26 Temperature response of $\mathrm{Ge}_{40} \mathrm{~S}_{60}$ covered silicon waveguide based temperature sensor, a) absolute slope of transmitted power as a function of time, b) absolute slope of transmitted power as a function of temperature.

7.1 Using refractive index matching UV-cured epoxy to bond fiber to the Si waveguide device. . . . . . . . . . . . . . . . . . . 147

7.2 a) Waveguide array with different ChG topping, b) output power versus temperature from the $\mathrm{ChG}$ covered $\mathrm{Si}$ waveguide, c) Temperature trend as a function of time using array structure. . . . . . . . . . . . 149

7.3 A multi-point temperature sensor chip incorporating inverse taper fiber couplers, 1-to-N power splitters, waveguides coated with different $\mathrm{ChG}$ glasses with varying $T_{c}$, and input and output radiation hardened optical fibers. . . . . . . . . . . . . . . . . . . 150

7.4 a) Transmission spectra of the temperature sensor for different temperatures, b) Linear relationship between the temperature and the resonance wavelength. . . . . . . . . . . . . . . . . . . 152

A.1 Beam blanker causes extra beam spots during idle time of the exposure.177

A.2 Different beam blanker setting in system file. . . . . . . . . . 178

A.3 Different beam blanker setting in system file. . . . . . . . . . . 178 
A.4 Image of patterned designs after fixing beam blanker issue, a) pattern with different beam dose, b) stitching. . . . . . . . . . . . . . . . . 180

A.5 Heavy contamination in $\mathrm{T} 1$ and $\mathrm{T} 2$ detectors. . . . . . . . . . . . . . 181

A.6 Beam current issue during patterning. . . . . . . . . . . . . . . 182

A.7 Testing beam issue on design in NPGS library. . . . . . . . . . . . . 182

B.1 The spin speed versus film thickness curves [http://microchem.com]. . 185

B.2 The spin speed versus film thickness curves for ZEP 520A [ZEON corporation]. . . . . . . . . . . . . . . . . 186

B.3 The spin speed versus film thickness curves for ZEP 520A [ZEON corporation]. . . . . . . . . . . . . . . . . . . 187

B.4 Schematic of Reactive Ion Etching (RIE) after EBL. a) EBL is performed on the resist, b) exposed resist is developed, c) the substrate without resist covering is etched down by plasma, and d) resist is removed. . . . . . . . . . . . . . . . . . . 190 


\section{LIST OF TABLES}

3.1 Summary of the measured complex refractive indices of synthesized glasses in amorphous and crystalline phases at $1550 \mathrm{~nm}$ wavelength. . 41

3.2 Measured complex refractive index of $\mathrm{Ge}_{40} \mathrm{Se}_{60}$ at different temperatures at $1550 \mathrm{~nm}$ wavelength. . . . . . . . . . . . . . . . . 42

4.1 Temperature response of Ge-S and Ge-Se tip coated optical fiber based temperature sensor. ...................... 65

4.2 comparison between performance of proposed optical fiber based temperature sensor with other studies. . . . . . . . . . . . . 70

5.1 Optimum design parameters obtained for Design 1. . . . . . . . 75

5.2 Amorphous phase waveguide loss of Design 1. . . . . . . . . . . 76

5.3 Crystalline phase waveguide loss of Design 1. . . . . . . . . . . . 78

5.4 Summary of losses for Design 1. . . . . . . . . . . . . . . . . . 79

5.5 Optimum design parameters obtained for Design 2. . . . . . . . . . . 80

5.6 Amorphous phase waveguide loss of Design 2. . . . . . . . . . . . 81

5.7 Crystalline phase waveguide loss of Design 2. . . . . . . . . . 83

5.8 Summary of losses for Design 2. . . . . . . . . . . . . 83

5.9 Optimum design parameters obtained for Design 3. . . . . . . . . . . 84

5.10 Amorphous phase waveguide loss of Design 3. . . . . . . . . . 85 
5.11 Waveguide loss of Design $3 . \ldots \ldots \ldots \ldots$

5.12 Summary of losses for Design $3 \ldots \ldots \ldots \ldots$

5.13 Optimum design parameters obtained for Design 4. . . . . . . . 87

5.14 Amorphous phase waveguide loss of Design 4. . . . . . . . 88

5.15 Waveguide loss of Design $4 \ldots \ldots \ldots$

5.16 Performance comparison between the 4 design architectures. . . . . . 90

5.17 Fundamental TM mode loss in Waveguide design 1 with in-house synthesized in-house inks . . . . . . . . . . . . . . . . . . . 98

5.18 Fundamental TM mode loss in Waveguide design 3 with in-house synthesized inks. . . . . . . . . . . . . . . . 105

5.19 Fundamental TM mode loss in Waveguide design 4 with in-house synthesized inks. . . . . . . . . . . . . . . . . 111

5.20 Comparison of TM mode losses in all waveguide design with in-house synthesized inks. . . . . . . . . . . . . . . . . . . 112

6.1 Sweep grating coupler parameters . . . . . . . . . . . . . . 118

B.1 PMMA detail information $\ldots \ldots \ldots \ldots \ldots \ldots$ 


\section{LIST OF ABBREVIATIONS}

\begin{tabular}{|c|c|}
\hline $\mathrm{AFM}$ & Atomic Force Microscope \\
\hline BOX & Buried Oxide \\
\hline BW & Bandwidth \\
\hline $\mathrm{CE}$ & Coupling Efficiency \\
\hline ChGs & Chalcogenide Glasses \\
\hline CMOS & Complementary Metal-Oxide Semiconductor \\
\hline $\mathrm{EBL}$ & Electron-Beam Lithography \\
\hline EME & EigenMode Expansion \\
\hline EM & Electromagnetic \\
\hline EMI & Electromagnetic Interference \\
\hline NPGS & Nanometer Pattern Generation System \\
\hline OFS & Optical Fiber Sensor \\
\hline OSA & Optical Spectrum Analyzer \\
\hline PIC & Photonic Integrated Circuits \\
\hline PMMA & Polymethylmethacrylate \\
\hline PVD & Physical Vapor Deposition \\
\hline RFI & Radio Frequency Interference \\
\hline RIE & Reactive Ion Etching \\
\hline RI & Refractive Index \\
\hline RTDs & Resistance Temperature Detectors \\
\hline SEM & Scanning Electron Microscopy \\
\hline $\mathrm{SiC}$ & Silicon Carbide \\
\hline SOI & Silicon-on-Insulator \\
\hline SP & Surface Plasmon \\
\hline SPPs & Surface Plasmon Polaritons \\
\hline SPR & Surface Plasmon Resonance \\
\hline TE & Transverse-Electric \\
\hline
\end{tabular}




$\begin{array}{ll}\text { TIR } & \text { Total Internal Reflection } \\ \text { TM } & \text { Transverse-Magnetic }\end{array}$

xxxiii 


\section{CHAPTER 1: \\ INTRODUCTION}

\subsection{Traditional Electronic Sensors Used in Extreme Environments}

One of the most frequently measured physical parameters is temperature. Often, temperature change can be an indicator of physical changes occurring in other parameters of interest. It is a fundamental parameter in many processes ranging from industrial manufacturing to life sciences. The operating environments inside nuclear reactors create extreme conditions for fuel and structural components, involving high temperature, neutron irradiation, and corrosive conditions. To observe material performance (microstructure, chemistry, mechanical, and other property changes with the changing conditions) while exposed to the reactor environment and to prevent disasters caused by structural failure due to high temperature, real-time and accurate sensing devices are required. Generation IV nuclear power plants operate at high temperatures up to about $1000{ }^{\circ} \mathrm{C}$ to achieve higher efficiency [1-3]. Precise temperature monitoring within this nuclear reactor is critical for its safety, stability, proper

functionality, and efficient operation over the long term operational lifetime [4-6].

During the research, development, and qualification stages of new reactor materials, structural components, and fuel, it is imperative to study their performance 
under specific test conditions before commissioning these materials or components. Such testing procedures require real-time temperature monitoring with high precision. The temperature range of interest for the fuel and structural component community varies quite significantly depending on the reactor technology evaluated. Typically, reactor fuel design teams strive to decrease fuel cladding temperatures, although the designs need to be robust enough to withstand higher temperatures during accident conditions. Realizing a sensor for such hazardous environments remains to be a tremendous engineering challenge. Usually, in any high radiation environment (e.g., reactor cladding, spent fuel pool), the temperature is continuously monitored using conventional resistance thermometry metal-based temperature measurement instruments like thermocouples, Resistance Temperature Detectors (RTD) or meltwire sensors $[6,7]$. The working mechanism of these non-optical sensors are based on variances in metal's resistance with temperature.

In nuclear power plants, mostly RTDs are used to measure the primary coolant temperature [8]. RTDs are thermal devices which contain an electrical resistance element referred to as the sensing element. Changes in its resistance value with temperature can be used to indirectly determine the temperature [9]. These sensors are considered as the most accurate resistance-based temperature sensors [10]. The current RTDs can accurately show the temperature up to about $400{ }^{\circ} \mathrm{C}$. The sensing element of industrial RTDs are mostly made of platinum [10]. Although RTDs give real-time data about temperature, RTDs need external current to measure the resistance change. Thus, with measuring current $I, I^{2} R$ heating inside the RTD device presents itself as a problem known as "self-heating" [6]. Moreover, gamma heating due to radiation absorption gives erroneous reading. RTDs are also the most expen- 
sive temperature sensors [11]. RTDs suffer from signal drift at high temperature, response-time degradation, reduced insulation resistance, and erratic output, which leads to frequent calibration requirements [8]. Estimating the coolant temperature profile requires many RTDs, which increases cabling and maintenance requirements.

Another commonly used metal-based temperature sensor is a melt-wire. In these sensors, known metal compositions with specific melting temperatures are placed in an irradiation test environment. A post-test examination on which wires melted and which did not can be used to determine the ambient temperature. One issue with this method is that the metal bars should have $99 \%$ purity [12] to achieve accurate results. Melt-wire sensors are inexpensive but do not allow for real-time monitoring of temperature, can only be checked post factum, and have low resolution on temperature.

For decades, high temperature resistant thermocouples have been used to monitor the temperature during the irradiation. However, these thermocouples need to be calibrated when exposed to temperatures above $1100{ }^{\circ} \mathrm{C}[13]$. A thermocouple is an assembly of two wires of different metals. These metals are joined at one end which is called a hot end, for temperature measuring, and the other end is considered as the cold junction which works as a reference at $0{ }^{\circ} \mathrm{C}$. Compared to melt-wires, thermocouples provide real-time reading of temperature. However, the output signal from a thermocouple is weak. Thermocouples are large in size, provide low resolution, are single point detectors and highly sensitive to common mode noises, which compromises their accuracy. Additionally, the performance of thermocouples and RTDs are affected by oxidation and attack from corrosive chemicals and the temperature readings drift significantly under long-duration exposure to high-temperature [2] and 
radiation [3], simultaneously during long-term operation $[2,14,15]$. These limit their operational life time. This often necessitates sensor recalibration due to transmutation from absorption of neutrons [7]. Thermometers are often required to operate in the presence of strong electromagnetic fields, while in contact with human patients, or to be interrogated over long distances. Sensors with metallic leads will experience eddy currents in such environments, which will create both noise and the potential for heating of the sensor, which in turn causes inaccuracy in the temperature measurement.

The desire to reduce sensor ownership cost and overcome the limitation of traditional (electrical) temperature sensors have attracted considerable interest in photonic temperature sensors within nuclear power plants as well as high energy physics accelerators and experiments [16-20]. Optical temperature sensors are instruments that convert external temperature stimuli into a detectable optical signal changes. These sensors provide several advantages compared to their electrical counterparts. Aging of electrical cables is a major concern in nuclear reactors. Electrical cables are degraded by temperature and ionizing radiation, which could cause mechanical property changes or cracking of insulators which could then end up with an electrical failure or even with ignition in the worst case. Radiation-hard optical fiber based sensors (OFSs) that possess low degradation and stability under harsh environments are best candidates for temperature monitoring in spaces where conventional sensors such as melt-wires and thermocouples are not well suited [21-25]. These sensors, which do not use metallic transducers to perform their conversion, allow for minimized heat dissipation by conduction and provide quick response. Since they are less perturbing to the environment, they have the potential for extreme accuracy [26]. 
Optical sensing advantages include low drift, high bandwidth, standoff sensing, and distributed sensing. These sensors offer more advantages over conventional electronic sensors as listed below [27-29].

- Easy incorporation into a wide variety of structures, including composite materials, with little interference due to their small size and cylindrical geometry

- Inability to conduct electric current.

- Immunity to electromagnetic interference (EMI) and radio frequency interference (RFI)

- Lightweight and high sensitivity.

- Multi-functional sensing capabilities such as strain, pressure, corrosion, temperature, and acoustic signals

- Resistant to high temperatures and chemically reactive environments

- High sensitivity for temperature monitoring and low attenuation signal transmission from within the nuclear reactor to an external control center

\subsection{Summary of Contributions}

In this research, innovative techniques to design and fabricate real-time and long-term direct measurement high temperature optical waveguide sensors (silicon and silica based) capable of operating at temperatures up to $600{ }^{\circ} \mathrm{C}$ are presented. Integrating these devices with the phase change properties of chalcogenide glasses (ChGs) facilitates precise determination of temperature profile, multiple-time use, and reversibility and radiation harden of the sensors for nuclear reactors. We show for the first time 
that unlike conventional optical fiber based high temperature sensors, our sensor, by simple capping fiber tips with temperature sensitive materials can measure high temperature in harsh environments. Also, we show that planar Si waveguides can perform as a temperature sensor if we cover the waveguide with chalcogenide glasses. The reversible characteristic of the chalcogenide glasses can transform single-time temperature sensors into multi-time use devices. Our sensors are low power consuming and cost effective devices. Fabrication of these compact size optical waveguide based temperature sensors is easy for integration in different structures of a nuclear facility. We can locate these sensors directly over the desired surface to measure temperature. Extending one single sensor to an array structure can be used to track the temperature inside harsh environments. The efforts devoted to innovative sensing techniques focus mainly on the following issues.

- The study of sensing mechanism

- Cost-effective design and implementation

- Material selection to fabricate the sensors

- Measure real-time temperature response of the sensors

- Packaging structure design of the sensing probe

- Sensor characterization set up

- Optimization of the sensor prototype and performance evaluation 


\subsection{List of Publications}

The above contributions, so far, has led to the following publications in prestigious journals and conferences:

\subsubsection{Journal Papers}

1. Badamchi, B., Ahmed Simon, A.-A., Mitkova, M., Subbaraman, H., "Chalcogenide Glass-Capped Fiber-Optic Sensor for Real-Time Temperature Monitoring in Extreme Environments," Sensors, Feb 2021.

2. Al-Amin Ahmed Simon, Bahareh Badamchi, Harish Subbaraman, Yoshifumi Sakaguchi, Maria Mitkova, "Phase change in Ge-Se chalcogenide glasses and its implications on optical temperature-sensing devices," Journal of Materials Science: Materials in Electronics, May 2020.

3. Al-Amin Ahmed Simon, Shah Rahmot Ullah, Bahareh Badamchi, Harish Subbaraman, Maria Mitkova, "Materials Characterization of Thin Films Printed with $\mathrm{Ge}_{20} \mathrm{Se}_{80}$ Ink," Microscopy and Microanalysis, Aug 2019.

\subsubsection{Conference Papers}

1. Sohel Rana, Bahareh Badamchi, Harish Subbaraman, Nirmala Kandadai, "A simple and cost-effective metal coating method for reflective long period grating sensors," Infrared Sensors, Devices, and Applications X, 2020.

2. Bahareh Badamchi, Nirmala Kandadai, Al-Amin Ahmed Simon, Maria Mitkova, Harish Subbaraman, "A novel high temperature optical waveguide sensor for nuclear reactors," 11th International Topical Meeting on Nuclear Plant Instrumentation, Control and Human Machine Interface Technologies (NPIC \& HMIT), 
2019.

3. Sohel Rana, Bahareh Badamchi, Binay Joshi, Harish Subbaraman, Nirmala Kandadai, "Towards Time Stable Fiber Optic Sensors for Harsh Environment," 11th International Topical Meeting on Nuclear Plant Instrumentation, Control and Human Machine Interface Technologies (NPIC \& HMIT), 2019.

4. Al-Amin Ahmed Simon, Karishmae Kadrager, Bahareh Badamchi, Harish Subbaraman, Maria Mitkova, "Temperature Sensing in Nuclear Facilities: Application of the Phase Change Effect of Chalcogenide Glasses," Nuclear Plant Instrumentation, Control, and Human-Machine Interface Technologies, 2019.

5. Ashley E Rivera, Sohel Rana, Nirmala Kandadai, Bahareh Badamchi, "Ellipsometry Measurement of Novel Optical Materials," Idaho Conference on Undergraduate Research, 2019.

\subsubsection{Showcase}

1. Al-Amin Ahmed Simon, Shah Mohammad Rahmot Ullah, Bahareh Badamchi, Harish Subbaraman, Maria Mitkova, "Inkjet Printing of Chalcogenide Glass,

" Graduate student showcase, Boise State University, 2019.

2. Bahareh Badamchi, Nirmala Kandadai, Al-Amin Ahmed Simon, Maria Mitkova, Harish Subbaraman, "A Novel High Temperature Sensor Architecture for Harsh Environments," Graduate student showcase, Boise State University, 2018.

\subsubsection{Patent}

- Maria Mitkova, Al-Amin Ahmed Simon, Shah Mohammad Rahmot Ullah, Bahareh Badamchi, Harish Subbaraman, "Thin Film Printed with Chalcogenide 
Glass Ink," 19975.104US01.

\subsection{List of Awards}

- Bahareh Badamchi, Nirmala Kandadai, Al-Amin Ahmed Simon, Maria Mitkova, Harish Subbaraman, "A Novel High Temperature Sensor Architecture for Harsh Environments," Graduate student showcase, Boise State University, 2018.

\subsection{Dissertation Outline}

The outline of the dissertation is presented below.

In Chapter 1, the introduction of traditional electronic sensors used in extreme environments and the need for research devices are presented.

In Chapter 2, background related to my research is presented. The first section includes a brief introduction of the theory on optical fibers and waveguides that will be relevant to understanding the results presented in later chapters. Next, optical fiber and planar optical waveguide working mechanisms and light coupling methods are introduced. Then, an introduction to surface plasmon polariton and plasmonic waveguide structures are presented. Also, the chalcogenide glass characteristics are explained.

Chapter 3 follows the work performed on Architecture 1. The proposed optical fiber-based high temperature sensor design is explained. The design is simulated in PhotonDesign software to demonstrate the performance of the sensor. The optimum dimension of the design is obtained. The performance of the sensing device in amorphous and crystalline phases of the synthesized ChGs is studied.

In Chapter 4, the fabrication process of a fiber-based temperature sensor is described. The process to prepare the fiber tip to deposit the $\mathrm{ChG}$ along with the 
depositing process is described in detail. Also, the characterization set-up built to characterize devices is presented. The temperature response of the sensor and data analysis are discussed in this chapter.

Chapter 5 focuses on the simulation of the silicon waveguide based temperature sensors with different in-house synthesized ChGs covering. The simulation results are used to optimize the design feature sizes to have a single-mode performance of the waveguides. The plasmonic effect of $\mathrm{ChG}$ in the crystalline phase is confirmed. Also, a proper waveguide length is selected to achieve a high extinction ratio in the temperature responses of the sensor.

In Chapter 6, the fabrication process of the $\mathrm{Si}$ ChG waveguide-based temperature sensor is described in detail. Two different testing set-ups, grating coupling and another for edge coupling bases devices are described. The temperature response of the proposed sensor is measured and results are discussed.

In Chapter, 7 discussion of the future research directions and proposed improvements on the temperature sensors are presented. We have shown proper packaging methods to be useful in industrial. The sensitivity study can be studied to confirm high sensitivity of the sensors.

In Chapter 8, we summarize the contents presented in the dissertation and provide an outlook of the future perspective in this integration approach. In the Appendix A, limitations of our EBL tool and modifications performed to improve performance is discussed. In Appendix B, the waveguide fabrication in details is introduced. 


\section{CHAPTER 2:}

\section{BACKGROUND}

\subsection{Optical Waveguides}

Optical waveguides are the fundamental components for transmitting light over distances ranging from tens or hundreds of micrometers (e.g. in integrated optical circuits and semiconductors lasers) to thousands of kilometers (e.g. optical fibers). The basic structure of a dielectric optical waveguide consists of a high refractive-index (RI) optical medium, called the core surrounded by low-index media, called the cladding. A variety of different integrated optical waveguide structures are used to confine light and transport energy at wavelength ranging from visible to mid-IR regions of the electromagnetic spectrum on a chip [30-32]. A guided wave propagates in the waveguide along its longitudinal direction. There are two different waveguide structures, a) nonplanar, and b) planar. In a nonplanar waveguide, the core is surrounded by cladding in all transverse directions. In a planar waveguide, the core is sandwiched between cladding layers. We will discuss these structures in detail in the following sections.

\subsubsection{Optical Fiber}

An optical fiber is basically a cylindrical dielectric waveguide with a circular cross section where a high-index waveguiding core is surrounded by a low-index cladding, 


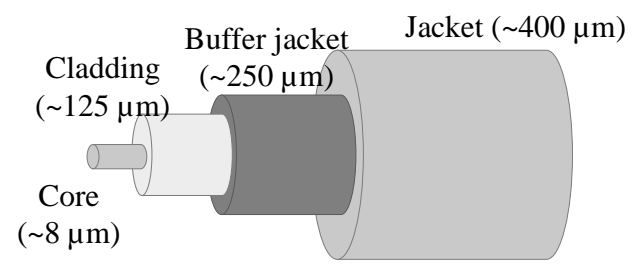

Figure 2.1: Schematic of optical fiber, showing typical diameter [33].

as shown in Figure. 2.1.

Optical fibers are usually made of silica $\left(\mathrm{SiO}_{2}\right)$. The RI profile is controlled through tuning the concentration and distribution of dopants. Alternatively, to take advantage of low-loss pure silica, the cladding can be doped with fluorine for a slightly lower index while the core contains undoped pure silica. Silica fibers are ideal for light transmission in the visible and near-infrared regions because of their low loss and low dispersion in these spectral regions. They are therefore suitable for optical communications and most laser applications in this range of the spectrum. Optical fibers have a wide range of applications. Owing to their low losses and large bandwidths, their most important applications are fiber-optic communications and interconnections [34]. Other important applications include fiber sensors, guided optical imaging, remote monitoring, and medical applications [35-37]. In addition, because optical fibers provide low loss $(\sim 0.1-0.2 \mathrm{~dB} / \mathrm{km})$ optical confinement over long distances, they also present unique conditions for many interesting nonlinear optical processes, which lead to such applications as optical soliton formation and propagation, optical pulse compression, and optical frequency conversion [38, 39].

The refractive index of the core is greater than the refractive of the cladding i.e $\left(n_{c o}>n_{c l}\right)$, thus light can propagate inside the high refractive index medium based on total internal reflection (TIR) as shown in Figure. 2.2. The TIR occurs when a 
ray of light strikes a boundary at an angle larger than the critical angle defined by $\sin ^{-1} \frac{n_{c l}}{n_{c o}}$ with respect to the normal of the surface [40].

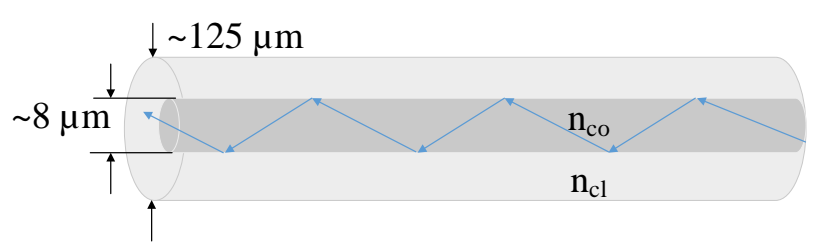

Figure 2.2: Propagation of light inside the fiber based on total internal reflection.

This criterion of TIR limits the required angle of incidence of a light beam which hits the fiber core from outside. Guidance is obtained only if the angle between the beam and the fiber axis is below a certain maximum which is defined as the numerical aperture (NA) of the fiber as shown in Equation 2.1.

$$
N A=\sqrt{n_{1}^{2}-n_{2}^{2}}
$$

Any beam with an incidence angle larger than $\sin ^{-1}(N A)$ is not guided by the core, and thus experiences high losses at the outer interface of the cladding.

The waveguide modes are electromagnetic field configurations (tangential between electrical (E) and magnetic $(\mathrm{H})$ field, denoted by linearly polarized $\mathrm{LP}_{l m}$ which maintain their intensity profile during propagation in the fiber core, where $2 l$ and $m$ are the number of maxima around a circumference and the number of maxima along the radius of the optical fiber, respectively. The normalized frequency or $V$-number determines the fraction of the optical power in a certain mode which is confined to the fiber core, which also determines the number of modes that a fiber can guide. It is 
defined as [41]:

$$
V=\frac{2 \pi a}{\lambda} N A
$$

where $\lambda$ is the wavelength of light in vacuum, $a$ is the fiber core radius, and $N A$ is the numerical aperture. A fiber with a large $V$-number can support multiple modes. A single-mode condition at which the $\mathrm{TE}_{01}$ and $\mathrm{TM}_{01}$ reach cut-off is achieved when the $V$-number value is less than 2.405. Such fibers support only the fundamental mode and are called single-mode fiber. The lowest order mode $\mathrm{HE}_{11}$ is denoted $\mathrm{LP}_{01}$ which is known as the fundamental mode of the fiber. Therefore, by changing the core size and the RI of the core/cladding, designing an optical fiber with a specific number of modes is possible.

\subsubsection{Planar Silicon Optical Waveguide}

Consider a straight optical waveguide propagating light along $z$ direction as the longitudinal direction. In a planar waveguide that has optical confinement in only one transverse direction, the core is sandwiched between cladding layers in only one direction, say the $x$ direction, with an index profile $n(x)$, as shown in Figure. 2.3 [34].

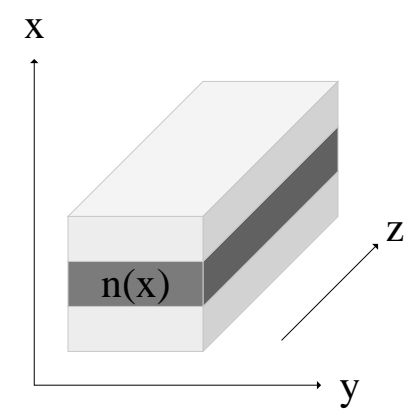

Figure 2.3: Planar waveguide of one-dimensional transverse optical confinement [34]. 
The core of a planar waveguide is also called the film, while the upper and lower cladding layers are called the cover and the substrate, respectively. This type of waveguide is called a slab waveguide. According to the refractive index distribution in the planar waveguide structure, these waveguides can be further classified as stepindex waveguides or graded index waveguides. A waveguide in which the refractive index profile has an abrupt change between the core and the cladding is called a stepindex waveguide [42], while the one in which its refractive index is depth-dependent along the $x$-axis, where the index profile varies gradually, is called a graded-index waveguide [43]. Figure. 2.4 shows examples of step-index and graded-index planar waveguides [44].

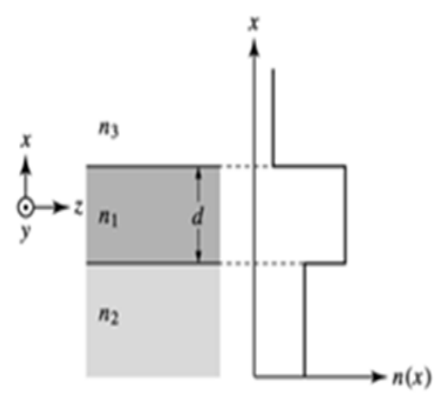

(a)

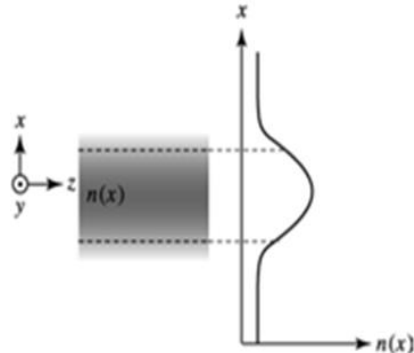

(b)

Figure 2.4: Index profiles of (a) a step-index planar waveguide and (b) a gradedindex planar waveguide [34].

Here, we describe the formation of modes with the ray picture in the slab waveguide [45], as shown in Figure. 2.5. Assume a plane wave propagating along the $z$-direction with inclination angle. The phase fronts of the plane waves are perpendicular to the light rays. The wavelength and the wavenumber of light in the core are $\frac{\lambda}{n_{1}}$ and $k_{1}=k n_{1}=\frac{2 \pi n_{1}}{\lambda}$, where $k$ and $\lambda$ are free-space propagation constants 


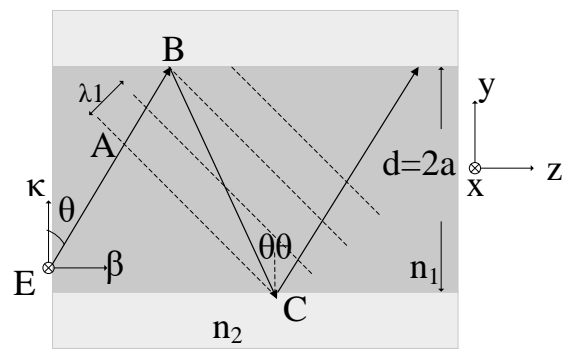

Figure 2.5: Light rays and their phase fronts in the waveguide [46].

and the wavelength, respectively. The direction of $k_{1}$ is at an angle $\theta$, normal to the core-cladding interface as shown in Figure. 2.5. Consider the phase difference between the two light rays belonging to the same plane wave. The phase difference between $A$ and $C$ corresponds to an optical path length $A B+B C$. In addition, light ray $B C$, propagating from point $B$ to $C$, have two total internal reflections at $B$ and $C$ (at the upper and lower core-cladding interfaces) which introduces further phase difference of $\phi$. The phase difference between $A$ and $C\left(\Delta \phi_{A C}\right)$ for constructive interference should be a multiple of $2 \pi$.

$$
\begin{gathered}
\Delta \phi_{A C}=k_{1}(A B+B C)-2 \phi=m(2 \pi) \\
A B+B C=B C \cos (2 \theta)+B C=B C\left(\left(2 \cos ^{2} \theta-1\right)+1\right)=2 d \cos (\theta)
\end{gathered}
$$

where $m=0,1,2, \ldots$ is an integer. Thus, the condition for wave propagation is

$$
k_{1} 2 d \cos (\theta)-2 \phi=m(2 \pi)
$$

It is apparent that only certain $\theta$ and $\phi$ values can satisfy this equation for a given 
integer $m$. But the phase change $\phi$ depends on $\theta$ and also on the polarization state of the wave (direction of the electric field, that is, $E_{x}$ here). For each $m$, there is one allowed angle $\theta_{m}$ and one corresponding $\phi_{m}$. Then, the waveguide condition is

$$
\frac{2 \pi n_{1}(2 a)}{\lambda} \cos \left(\theta_{m}\right)-\phi_{m}=m \pi
$$

which $\phi_{m}$ indicates that $\phi$ is a function of the incidence angle $\theta_{m}$. This equation is called a phase-matching condition of the waveguide. The optical field distribution that satisfies this equation is called the mode. The integer $m$ identifies these modes and is called the mode number. For the lowest mode, $m=0$, the wave travels axially and is called the fundamental mode; the other modes, having larger angles, are higherorder modes $m \geq 1$. We can have up to a certain number of modes allowed in the waveguide. According to phase matching condition and total internal reflection, the mode number must satisfy [47]

$$
m \leq \frac{2 V-\phi}{\pi}
$$

which $V$, called the $V$-number, defined by

$$
V=\frac{2 \pi a \sqrt{n_{1}^{2}-n_{2}^{2}}}{\lambda}
$$

Suppose that for the lowest mode, the propagation is due to a glazing incidence at $\alpha_{m} \rightarrow \pi / 2$, then $\phi \rightarrow \pi$, and $V=\frac{m \pi+\phi}{2}$ or $\pi / 2$. Thus, when $V<\pi / 2, m=0$ is the only fundamental mode propagating along the dielectric slab waveguide, which is then termed as a single-mode planar waveguide. The free-space wavelength $\lambda_{c}$ 
that leads to $V=\pi / 2$ is the cutoff wavelength, and above this wavelength, only one fundamental mode will propagate.

Figure. 2.6 shows two of the possibilities for the electric field direction of a waveguide traveling along the core-cladding boundary. The electric field is perpendicular to the plane of incidence (plane of paper) in Figure. 2.6a as indicated by $E_{\perp}$, is along $y$, so that $E_{\perp}=E_{y}$. The modes are associated with $E_{\perp}$ are termed transverse electric field modes, denoted by TE, and actually perpendicular to the direction of propagation, z. In other words, TE means that there is no electric field component in the direction of propagation. The magnetic field is perpendicular to the plane of incidence as indicated by $B_{\perp}$, as shown in Figure. 2.6b. In this case, the electric field is parallel to the plane of incidence and is denoted by $E_{\|}$. The modes associated with the $E_{\|}$ field have a magnetic field $B_{\perp}$ that is perpendicular to the direction of propagation and are termed transverse magnetic field modes, denoted by TM.

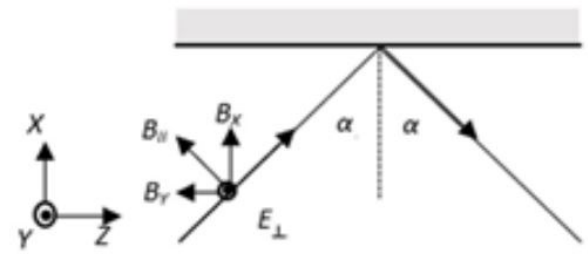

(a)

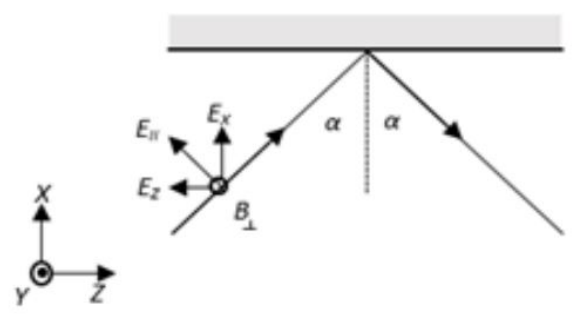

(b)

Figure 2.6: Possible modes can be classified in terms of (a) transverse electric field (TE), and (b) transverse magnetic field (TM).

\subsubsection{Channel Waveguides}

Channel waveguides known as 2D waveguides are those which have a higher refractive index in the core compared to substrate. A channel waveguide includes buried 
channel waveguides, strip-loaded waveguides, ridge waveguides, rib waveguides, and the diffused waveguides, shown in Figure. 2.7.

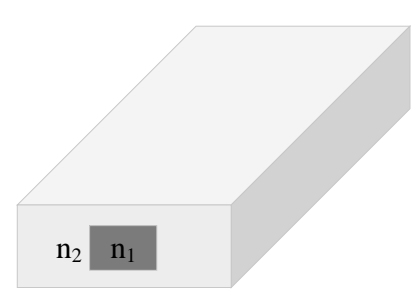

(a)

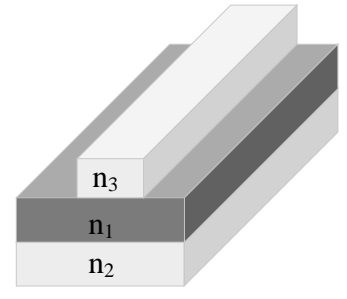

(b)

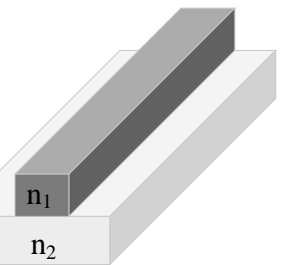

(c)

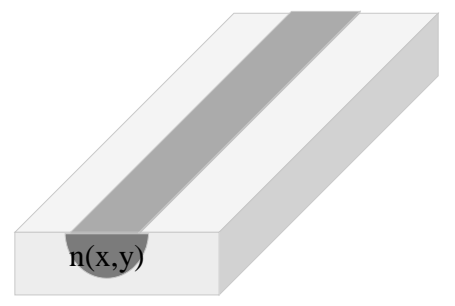

(d)

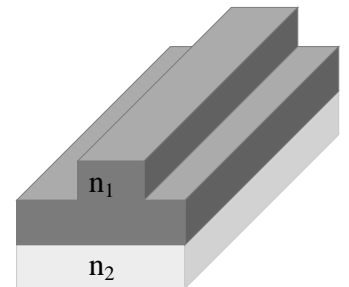

(e)

Figure 2.7: Channel waveguides, a) Buried channel waveguide, b) Strip-loaded waveguide, c) Ridge waveguide, d) Diffused waveguide, e) Rib waveguide.

A buried channel waveguide is formed with a high-index waveguiding core buried in a low index surrounding medium. The waveguiding core can have any crosssectional geometry, though it is often intended to have a rectangular shape, as shown in Figure. 2.7a. A strip-loaded waveguide is formed by loading a planar waveguide, which already provides optical confinement in the $x$ direction, with a dielectric strip of index $\mathrm{n}_{3}<\mathrm{n}_{1}$ or a metal strip to facilitate optical confinement in the $y$ direction, as shown in Figure. 2.7b. The waveguiding core of a strip waveguide is the $\mathrm{n}_{1}$ region under the loading strip. A ridge waveguide has a structure that looks like a strip waveguide, but the strip, or the ridge, on top of its planar structure has a high index and is actually the waveguiding core. A ridge waveguide has strong optical 
confinement because it is surrounded on three sides by low-index air (or cladding material) as shown in Figure. 2.7c. A diffused waveguide, shown in Figure. 2.7d, is formed by creating a high-index region in a substrate through diffusion of dopants, such as Lithium Niobate $\left(\mathrm{LiNbO}_{3}\right)$ waveguide with a core formed by Titanium (Ti) diffusion. Because of the diffusion process, the core boundaries in the substrate are not sharply defined. However, a diffused waveguide also has a thickness d defined by the diffusion depth of the dopant in the $x$ direction and a width w defined by the distribution of the dopant in the $y$ direction. A rib waveguide has a structure similar to that of a strip or ridge waveguide, but the strip has the same index as the high index planar layer beneath it and is part of the waveguiding core. A rib waveguide is shown in Figure. 2.7e.

\subsubsection{Surface Plasmon Waveguiding}

Integrated photonic sensors can easily be designed with pure dielectric materials, noble metals, and a combination of both. Dielectric optical sensors are low loss and can easily be developed by the well-known CMOS fabrication technology. However, dielectric materials in combination with metals forms a novel type of waveguide where the guided light mode is a combination of dielectric guided wave and excited surface plasmon polaritons (SPPs). It can be termed as a hybrid or composite plasmonic waveguide. This allows for sub-wavelength light confinement which can be used for enhanced light-matter interactions $[48,49]$. This characteristic makes realizing nano photonic circuits at subwavelength sizes possible which can overcome the diffraction limit [49]. Although, compared to dielectric waveguides, plasmonic devices can confine the light into smaller volumes, and has greater sensitivity which enable fabricating small footprint devices, but the presence of lossy material (metal) introduces modal 
losses [48-50]. In order to realize plasmonic or the subwavelength optical waveguides, we first need to understand SPPs.

The first observation of the phenomenon of surface plasmon resonance (SPR) dates back to 1902, when Wood [51] reported the "uneven distribution of light in a diffraction grating spectrum" while he observed patterns of unusual dark and bright bands in the light reflected from a metal backed diffraction grating. Around the same time, in 1907, Zenneck theoretically formulated a special surface wave solution to the Maxwell's equation and demonstrated that the radio frequency surface electromagnetic waves occur at the boundary of two media when one medium is either lossy dielectric or a metal and the other is loss free [52]. He also suggested that the lossy part of the dielectric constant is responsible for the binding of the electromagnetic waves to the interface.

Polaritons are considered as quasi-particles resulting from the strong exchange of energy between electromagnetic wave and excitation in a material e.g. photonelectron coupling at optical frequencies. When electromagnetic fields are coupled to the oscillation of electron plasma of a conductor in a dielectric-conductor interface, electromagnetic surface waves are excited and propagate along the interface [53]. These surface waves are evanescently confined in the perpendicular direction and are known as SPP waves [54]. The quantum of these oscillations is referred to as surface plasmon (SP) (also a SP wave or a SP mode). The optical properties of metals and noble metals in particular have been discussed by numerous authors [55-57]. The optical properties of metals can be described by a complex dielectric constant that depends on the frequency of the light. Nobel metals possess a dense assembly of negatively charged free electrons (free electron charge density $\sim 10^{23} \mathrm{~cm}^{-2}$ ) in an equally 
charged positive ion background. Since positive ions possess an infinitely large mass compared to that of free electrons, therefore, according to the Jellium model, these ions can be termed as a perpetually constant positive background in which free electrons can easily move from one point to the other in such a fashion that the condition for quasi-neutrality is always fulfilled. The total charge density inside the metal always remains zero. SPP waves in metallic waveguides and metal nanostructures open the possibility to confine and guide optical waves on the nanometer scale [54].

The SPs are accompanied by a longitudinal (TM-polarized) electric field, which decays exponentially in metal as well as in dielectric medium. Due to this exponential decay of field intensity, the field has its maximum at the metal-dielectric interface itself. Both crucial properties of SPs being TM-polarized and exponential decay of electric field are found by solving the Maxwell's equations. The Maxwell's equations are given as:

$$
\begin{gathered}
\nabla \cdot D=\rho_{e x t} \\
\nabla \cdot B=0 \\
\nabla \times E=-\frac{\partial B}{\partial t} \\
\nabla \times H=J_{e x t}+\frac{\partial D}{\partial t}
\end{gathered}
$$

where $D, B, E$ and $H$ represent the four macroscopic fields; namely dielectric displacement, magnetic induction, electric field, and magnetic field respectively; while $\rho_{\text {ext }}$ and $J_{\text {ext }}$ represent the external charge and current densities, respectively. Two 


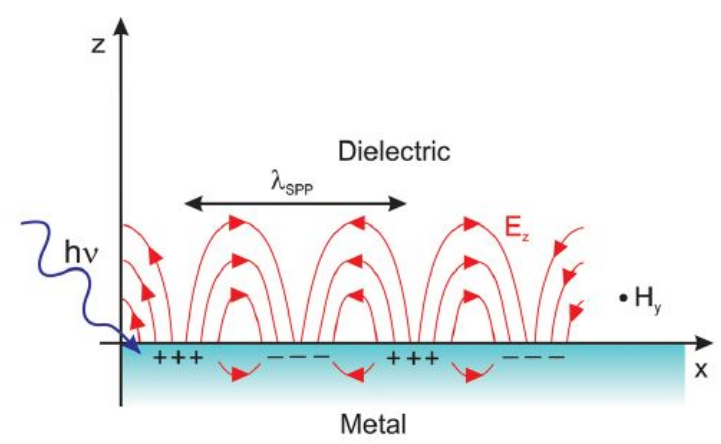

Figure 2.8: A schematic of the electromagnetic wave and surface charges at a metal-dielectric interface with semi-infinitely extended media on either side [58].

constructive relations relates $E$ and $H$ to $B$ and $D$ through $D=\epsilon \epsilon_{0} E$ and $B=\mu \mu_{0} H$ equations where $\epsilon$ is relative dielectric permittivity and $\mu$ is magnetic permeability of the medium. The $\epsilon_{0}=8.85 \times 10^{-12} \mathrm{~F} / \mathrm{m}$ and $\mu_{0}=4 \pi \times 10^{-7} \mathrm{H} / \mathrm{m}$ in a vacuum, respectively. For the kind of geometry shown in Figure. 2.8, the set of solutions $E_{x}$, $H_{y}$, and $E_{z}$ correspond to TM modes and $H_{x}, E_{y}$, and $H_{z}$ correspond to TE modes. The corresponding TM and TE wave equations are given by

$$
\begin{aligned}
& \frac{d^{2} H_{y}}{d z^{2}}+\left(k_{0}^{2} n^{2}-\beta^{2}\right) H_{y}=0 \\
& \frac{d^{2} E_{y}}{d z^{2}}+\left(k_{0}^{2} n^{2}-\beta^{2}\right) E_{y}=0
\end{aligned}
$$

where $k_{0}=2 \pi / \lambda_{0}$ is the propagation constant of the incident electromagnetic wave in free space and $\beta$ is that of the propagating wave in the medium. The propagating wave solutions confined to the interface, for the modes with exponential decay in $z$-direction be given as:

For $z>0$ :

$$
H_{y}=B e^{-\sqrt{\beta^{2}-k_{0}^{2} \epsilon_{d} z}}
$$




$$
E_{y}=A e^{-\sqrt{\beta^{2}-k_{0}^{2} \epsilon_{d} z}}
$$

For $\mathrm{Z}<0$ :

$$
\begin{aligned}
& H_{y}=D e^{-\sqrt{\beta^{2}-k_{0}^{2} \epsilon_{m} z}} \\
& E_{y}=C e^{-\sqrt{\beta^{2}-k_{0}^{2} \epsilon_{m} z}}
\end{aligned}
$$

Where $\epsilon_{m}=\epsilon_{m 1}+i \epsilon_{m 2}$ is dielectric constant of the metal and $\epsilon_{d}$ is dielectric constant of dielectric medium. By applying two boundary conditions for TE waves:

(i) $E_{y}$ (boundary $\left.(z=0)\right)=$ continuous

(ii) $H_{x}$ (boundary $\left.(z=0)\right)=$ continuous

We have $A=C$. Then substitute in the equations

$$
\begin{gathered}
\sqrt{\beta^{2}-k_{0}^{2} \epsilon_{m}}=\sqrt{\beta^{2}-k_{0}^{2} \epsilon_{d}} \\
A^{2} k_{0}^{2}\left(\epsilon_{d}-\epsilon_{m}\right)=0
\end{gathered}
$$

Since $\epsilon_{m}$ and $\epsilon_{d}$ are of opposite signs, the term in brackets cannot be zero. This implies that $A=0$. Thus no surface modes exist for TE polarization. Then by applying boundary conditions for TM waves

(i) $E_{x}$ (boundary $\left.(\mathrm{z}=0)\right)=$ continuous

(ii) $H_{y}$ (boundary $\left.(\mathrm{z}=0)\right)=$ continuous 
By applying these conditions, the obtain dispersion relation for an SPP at the interface is:

$$
\begin{gathered}
\frac{-\sqrt{\beta^{2}-k_{0}^{2} \epsilon_{d}}}{\epsilon_{d}}=\frac{-\sqrt{\beta^{2}-k_{0}^{2} \epsilon_{m}}}{\epsilon_{m}} \\
\beta=k_{0} \sqrt{\frac{\epsilon_{d} \epsilon_{m}}{\epsilon_{d}+\epsilon_{m}}}
\end{gathered}
$$

The charge distributions and field profiles, also shown in Figure. 2.8, point towards the following two important properties of SPs:

1. The SP mode is coupled to both the dielectric and the metal as the field of the SPs is in both the media.

2. The field in the metal decays more rapidly exponentially than in the dielectric medium with distance being subject to ohmic and radiation losses [59], as is symbolically shown by a number of electric fields. A more accurate and quantitative variation of the field profile is presented later in Figure. 2.9.

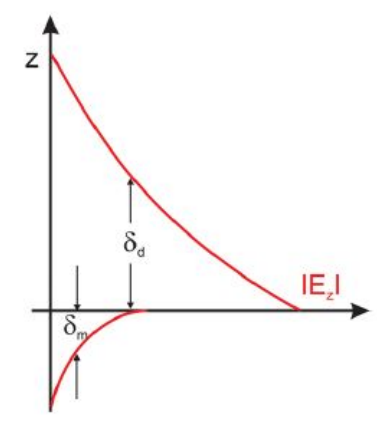

Figure 2.9: Field profile at a metal-dielectric interface [58].

The distance in the material from the interface over which the field amplitude falls to $\frac{1}{e}$ of its value at the interface is called the penetration depth $(\delta)$ of the SP wave [53]. 
Real metals have a complex permittivity $\left(\epsilon_{m}=\epsilon_{m 1}+i \epsilon_{m 2}\right)$ and the imaginary part of the permittivity is not negligible. For noble metals like gold and silver at higher optical frequency regime, however, $\left|\epsilon_{m 2}\right| \ll\left|\epsilon_{m 1}\right|$. The penetration depths into dielectric $\left(\delta_{d}\right)$ and into metal $\left(\delta_{m}\right)$ can be written as:

$$
\delta_{d}=\frac{\lambda_{0}}{2 \pi}\left(\frac{\epsilon_{d}+\epsilon_{m 1}}{\epsilon_{d}^{2}}\right)^{1 / 2}
$$

and

$$
\delta_{m}=\frac{\lambda_{0}}{2 \pi}\left(\frac{\epsilon_{d}+\epsilon_{m 1}}{\epsilon_{m 1}^{2}}\right)^{1 / 2}
$$

The field associated with the SP wave can be written as:

$$
E=E_{0} \exp \left[+i\left(k_{x} x \pm k_{z} z-\omega t\right)\right]
$$

where + and - signs are for $z \geq 0$ and $z \leq 0$, respectively, and the propagation constant $k_{z}$ is imaginary which signifies that the field decays exponentially. The wave-vector $k_{x}$ lies parallel to the $x$-axis and is given by $k_{x}=\beta$. The field amplitude decays exponentially in both the metal and the dielectric medium. The field amplitude decay in metal is faster than that in the dielectric medium. This can be attributed to the imaginary part of the metal dielectric function which introduces losses. The penetration depth termed as the skin depth in the dielectric medium is much larger than that in the metal. The penetration depth in the dielectric gives us a measure of the length over which SP is sensitive to the changes in the refractive index of the dielectric medium, while that into the metal tells us about the thickness of the metal film required for the coupling of light incident from the other interface of the metal 
film.

The length over which the intensity of the SP wave decreases to $\frac{1}{e}$ of its maximum value in the dielectric region is called the propagation length of the $\mathrm{SP}\left(L_{s p}\right)$ [53]. Since the dielectric constant of the metal, $\epsilon_{m}$, is a complex number, the propagation constant of the SP wave, $k_{s p}$ supported by the metal-dielectric interface will also be a complex number with real and imaginary parts $k_{x}^{\prime}$ and $k_{x}^{\prime \prime}$ i.e.:

$$
k_{s p}=k_{x}=k_{x}^{\prime}+i k_{x}^{\prime \prime}
$$

where:

$$
\begin{gathered}
k_{x}^{\prime}=\frac{\omega}{c}\left(\frac{\epsilon_{d} \epsilon_{m 1}}{\epsilon_{d}+\epsilon_{m 1}}\right)^{1 / 2} \\
k_{x}^{\prime \prime}=\frac{\omega}{c}\left(\frac{\epsilon_{d} \epsilon_{m 1}}{\epsilon_{d}+\epsilon_{m 1}}\right)^{1 / 2} \frac{\epsilon_{m 2}}{2 \epsilon_{m 1}^{2}}
\end{gathered}
$$

Therefore, the intensity of the SP wave propagating along the interface decreases as $e^{\left(-2 k_{x}^{\prime \prime} x\right)}$. Thus the propagation length is given by:

$$
L_{s p}=\left(2 k_{x}^{\prime \prime}\right)^{-1}
$$

The metals having a large (negative) value of the real part of the dielectric constant and a small value of the imaginary part possess larger propagation lengths. The propagation length suggests the limiting size of the photonic/plasmonic structure one can have. 


\subsubsection{Coupling to Waveguide}

Coupler devices have been developed to overcome challenges in power injection from off-chip sources through single mode optical fibers to waveguides in silicon photonics. Whilst the reduction in waveguide dimensions enhances the performance of the photonic circuit and the packing density, it makes coupling of light to/from the Si single mode waveguide with $220 \times 500 \mathrm{~nm}^{2}$ core cross section circuit very difficult, particularly to/from standard optical fibers with fundamental Gaussian shape modes that typically have mode field diameter (MFD) of 10-11 $\mu \mathrm{m}$. Direct coupling of the fiber and such a small silicon waveguide results in high coupling losses $(\sim 20 \mathrm{~dB})$ due to very different thicknesses (Figure. 2.10) and refractive indices. Thus, the major challenge in achieving an efficient fiber-to-chip coupling is the mismatch between the fiber mode-size and mode-index to those of the Si-waveguide modes on a photonic chip [60].
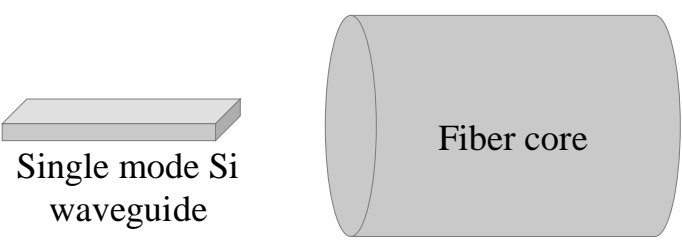

Figure 2.10: Mode mismatch between Si waveguide and optical fiber.

An efficient coupler device has to overcome several limiting processes to achieve maximal coupling. These processes inhibit transfer of power between the source and the waveguide. Coupling losses can arise from, for example, reflections in dielectric boundaries, different sizes and shapes of the fields, waveguide scattering, or back coupling. Some of these phenomena can be combined into single processes, which 
enables writing the following equation:

$$
p_{c p l}(d B)=p_{i n}(d B)-\Gamma(d B)-10 \log \left(1-|R|^{2}\right)
$$

where $\Gamma$ denotes losses due to electric field mismatch and $1-|R|^{2}$ term takes into account the losses due to reflections [61]. Mismatch term $\Gamma$ contains both the loss due to mismatch in field dimensions and in the shapes of the fields.

A variety of techniques exist for performing the coupling task, the most common being edge coupling (end-fire coupling and butt coupling), prism coupling, and grating coupling $[44,45,47]$. The principles of these four coupling techniques are shown schematically in Figure. 2.11.

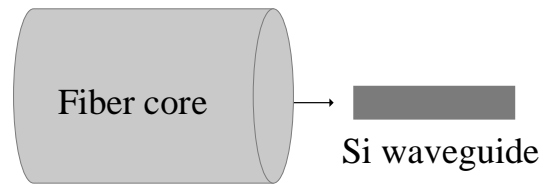

(a)

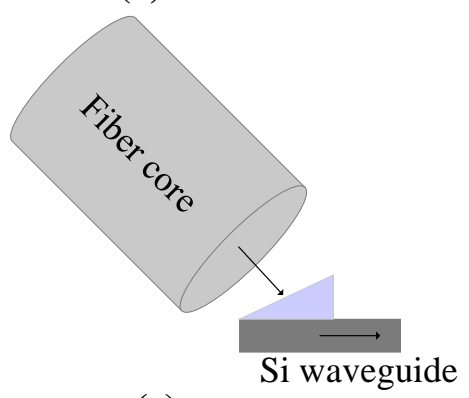

(c)

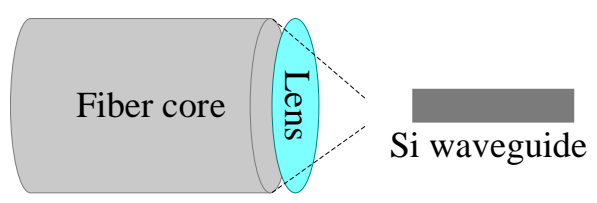

(b)

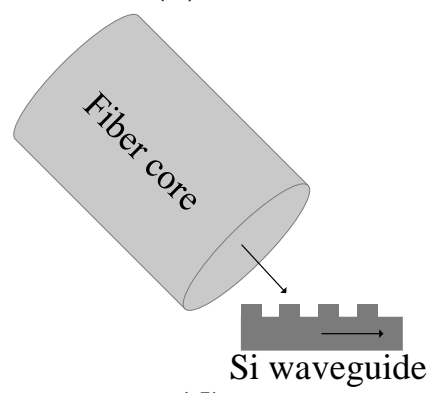

(d)

Figure 2.11: Schematic of a) butt coupling, b) end-fire coupling, c) prism coupling, d) grating coupling.

End-fire [62] and butt coupling are very similar, involving coupling light from an external source to an on-chip SOI waveguide by simply aligning and focusing a free 
space beam on-to the cleaved facet of the chip (where the waveguide edge is). The distinction that is usually made between these two methods is that butt coupling involves simply 'butting' the two devices or waveguides up to one another, such that the mode field of the 'transmitting' device falls onto the end face of the second device; whereas end-fire coupling incorporates a lens to focus the input beam onto the end face of the 'receiving' device. Therefore light is introduced into the end of the waveguide, and can potentially excite all modes of the waveguide [63].

\subsubsection{Prism coupling}

Free-space to waveguide coupling can also be achieved by total internal reflection of the beam from a facet of a high refractive index prism. This scheme is also known as evanescent coupling because it makes use of the evanescent wave penetrating through the small gap between the prism and the waveguide. A prism can focus and reshape the incident light and couple it to a waveguide at a specific angle. The high index prism, in the direction of the waveguide propagation has sufficient momentum to match the waveguide mode index, thus coupling to the corresponding propagating mode inside the waveguide can be achieved. The prism lies near the top surface of a waveguide separated by a small gap. The gap is essential because the technique is based on refractive index differences between four materials. Prism, gap, waveguide and cladding indices have the $n_{\text {prism }}>n_{\text {waveguide }}>n_{\text {cladding }}>n_{\text {gap }}$ [61]. The conditions of coupling are such that the material from which the prism is made should have a higher refractive index than the waveguide; this seriously restricts the possibilities, particularly for silicon which has a high refractive index of 3.5. There are materials available, however, such as germanium which could be used, although other 
limitations mean that the technique is still inferior to alternative techniques. The limitations are that prism coupling can damage the surface of the waveguide. Using prism to couple the light is not suitable if a surface cladding is to be used. Prisms are best suited to couple the light to planar waveguides, but not proper to material systems that utilize rib waveguides such as silicon technology. For the purposes of semiconductor waveguide evaluation, prism coupling is not particularly useful.

To overcome significant mismatch of the size and effective index of the modes between the fiber and SOI waveguides, a tapered mode converter is attached to the end of the SOI waveguide. Tapers utilize the gradual change of the effective refractive index experienced by the input beam. The gradual change guarantees that no considerable reflection occurs and that the incident beam is slowly adapted to the waveguide [64]. Change in the refractive index is tolerant to changes in the wavelength, which results in high coupling efficiency over broad bandwidth [61,64]. Inverse tapers offer benefits over those achievable by conventional taper structures [65]. The schematic of an inverse taper is shown in Figure. 2.12. These tapers can also become narrower towards the input to increase the mode size which results in a better overlap with the incoming fiber mode. The end point of an inverse taper can be several tens of nanometers thin [66]. An inverse taper coupler is the most popular scheme for such mode converting in $\mathrm{Si}$ photonics to achieve enhanced fiber-to-chip coupling due to their compactness and broadband properties. Inverse tapers have been demonstrated to achieve losses below $1 \mathrm{~dB}$ [61]. Inverse technique can also reduce the length of the taper to $200 \mu \mathrm{m}$. The short length of the taper a significant improvement in inverse taper technology because one limiting factor of the tapers in miniaturized circuits is their length [65]. 


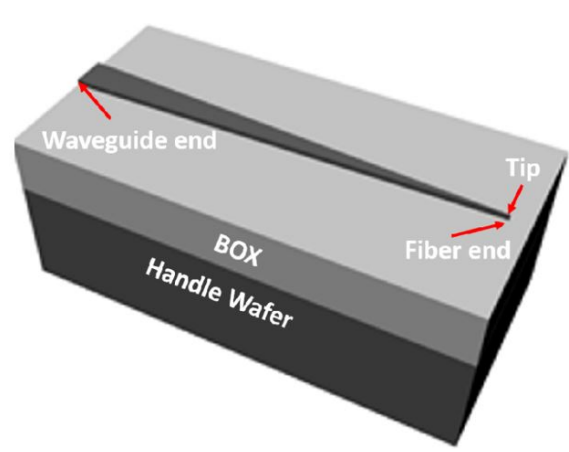

(a)

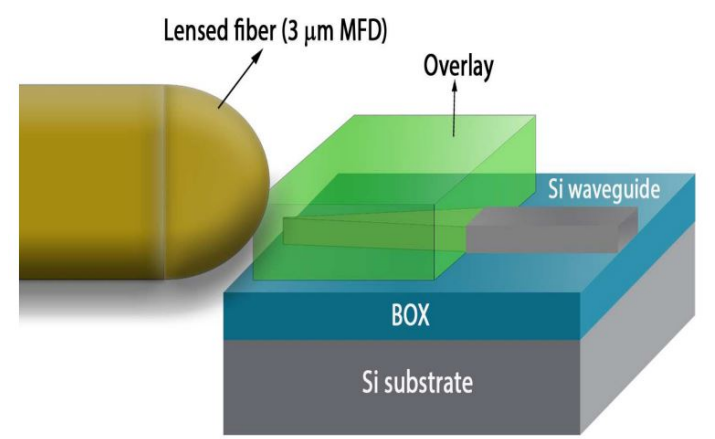

(b)

Figure 2.12: Schematic of a) an inverse taper [67], b) edge coupler using inversetapers.

\subsubsection{Grating coupler}

Grating couplers can solve the problems of coupler length and restrictions in position. They serve as a typical realization of a coupler device besides lateral tapers. In such couplers, the diffraction gratings are designed to provide additional momentum to the incoming beam of light (released from fiber) to match to the momentum of the waveguide mode. Often, to ensure unidirectional coupling, the grating teeth are designed to achieve maximum coupling for an angled incidence using the phase match condition. In general, gratings are used to find the spectra of light sources; however, they can also be used for the excitation of SP [53]. The use of a grating as a coupler is possible due to the interaction with a periodic lattice and the incoming light. They are compact, generally $12 \mu m \times 12 \mu m$ sized devices, which can be located in any part of a circuit $[61,68]$. Coupling in these devices is achieved through diffraction, which arises from light interacting with periodic alterations of the waveguide surface (diffraction grating). Diffraction can change propagation direction of incident light. Therefore, light in a grating coupler can turn in an angle that corresponds to one of 
the waveguide modes to be excited. Formally, this change of propagation is described by matching the phases of the incident radiation and the waveguide mode as shown in Figure. 2.13.

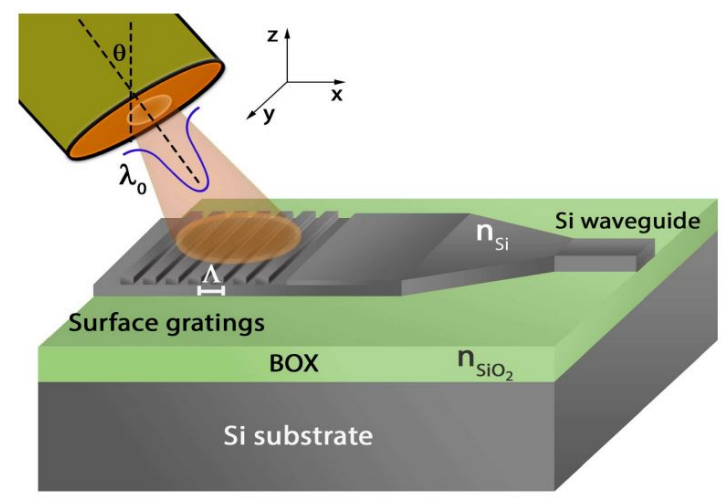

Figure 2.13: Coupling light through grating coupler to an SOI waveguide [69].

The requirement for the phase matching sets a condition for the grating dimensions. This condition turns into the strong frequency dependence [61] of grating couplers, because light diffracted from all grating elements have to be matched to the waveguide mode [70]. Narrow bandwidth, back coupling and reflections are limiting factors of the grating couplers. Particularly, a grating acts as surface roughness in the waveguide. Therefore, light can leave the grating in many directions. In SOI structure, down scattered light can interfere with the waveguide light, which leads to a dependency of the coupling efficiency from the bottom oxide thickness [71]. Coupled light can also reflect from the grating and be scattered back out of the waveguide [61]. Furthermore, grating operation is limited by strong polarization dependence, complex fabrication and requirements for precise alignment for coupling. However, these limitations can be avoided by optimizing the grating structure. Grating couplers have achieved coupling efficiencies of over $50 \%$ of the incident power. Efficiencies as high 
as $80 \%$ can be achieved by alteration of the cover medium, bottom oxide thickness, and the element shapes, and utilizing a bottom reflector beneath the grating.

As the proposed novel real-time, reusable and reversible sensor for harsh environments is achieved through integrating the photonic properties of optical waveguides and the phase change properties of chalcogenide glasses $(\mathrm{ChG})$, the following section provides a brief introduction to ChGs.

\subsubsection{Chalcogenide Glasses}

Chalcogenide glasses (ChGs) are inorganic amorphous compounds containing one or more of the chalcogen elements (group 6a of the periodic table), such as Sulphur, Selenium, and Tellurium but excluding Oxygen, covalently bonded to other elements such as As, Ge, Sb, Ga, Si, or P. The chalcogen elements with other metal or nonmetal elements exhibit unique optical nonlinear and mid-infrared properties such as wide infrared transparency windows. The optical band gap of ChGs lies in the visible or near-IR regions as the inter-atomic bonds are typically weaker than in oxide glasses. ChGs are composed of heavier atoms when compared to oxide glasses and exhibit low bond vibration energies and low phonon energies. This enables excellent transparency in the infrared wavelength regime 0.8-16 $\mu \mathrm{m}$ that covers the characteristic absorption peaks of several chemical and biological species. This characteristic makes them attractive for use in infrared-transmitting optical fibers, and as waveguides for optical sensors and telecommunications. They are easy to synthesize in bulk and thin film forms and their compositional flexibility allows tuning of optical properties, such as refractive index, making them ideal for infrared photonics. These glasses are widely used as phase change materials for microphotonic applications. One of the most striking properties of ChGs is their photosensitivity - a propensity for the chemical 
bonds to change when exposed to light with a wavelength near the band edge. Similar changes can also be produced by exposure to heat, X-rays or electron/ion beams. Two important parameters that we focus on to design the temperature sensor are:

A) Phase change phenomenon (amorphous to crystalline): These glasses with compositional flexibility also demonstrate ultra-fast crystallization rate and large optical properties contrast between their amorphous (disordered) and crystalline (ordered) phase, making them ideal for infrared phase change photonic applications [27-29, 72-74]. These glasses are easy to synthesize in bulk and in thin-film form. ChGs undergo a reversible solid-solid phase transition and becomes crystalline at a certain well-documented temperature which is called crystallization temperature $\left(\mathrm{T}_{c}\right)$.

When ChGs are heated, there are three temperatures of significant consideration which are related to change in material's property: (1) glass transition temperature $\left(T_{g}\right)$, (2) crystallization onset temperature $\left(T_{o}\right)$, and $(3)$ glass peak crystallization temperature $\left(T_{c}\right)$. The glass transition temperature $\left(T_{g}\right)$ is the main characteristic of a glass. It is related to the onset of fluidity. At this temperature, the compositional network is broken and the building blocks are macroscopically mobile. Crystallization onset temperature $\left(T_{o}\right)$ or critical transition temperature is the temperature at which ChG begins phase transition from a glassy to a crystalline phase. At higher temperature, melting occurs at the melting point of the glass $\left(T_{m}\right)$. This transition from amorphous to crystalline drastically changes the material's conductivity, refractive index, absorption coefficient and extinction coefficient, which can be utilized as a reference to measure the temperature. Assuming ideal behavior from ChG glasses, below the crystallization temperature, in their amorphous state, ChG glasses demon- 
strate good dielectric behavior. When the temperature of the ambient is above the crystallization transition temperature of $\mathrm{ChG}$, the material crystallizes and exhibits excellent conductive characteristics. At the onset of crystallization, the nucleation process is fast and switching occurs within 80-100 nanoseconds [73-76]. In that aspect, Ge-Se and Ge-S binary ChGs are promising candidates because of their high crystallization temperature $\left(T_{c}\right)$, stable glass forming ability in a wide compositional region, the high difference in optical parameters and resistance between amorphous and crystalline phase. Our interest is driven by the need to resolve high temperature monitoring up to $600 \mathrm{~K}$. The required target temperature response is achieved through fine tuning of the composition of the active ChG using multicomponent systems since the crystallization temperature is a very accurate materials characteristic $[77,78]$.

Ovshinsky [79] found that phase change ChGs can be reversibility cycled between a highly resistive and a conductive state. The ChG can be re-converted to its initial amorphous condition through thermodynamic conditions by applying short optical [80] or electrical pulses with relatively low energies as low as femtojoules $[81,82]$. These materials play an important roles in evolution of plasmonic [83] and dielectric waveguides delivering a variety of switchable, tunable, and reconfigurable optical applications $[81,84]$. In a representative demonstration, Michel et al. used GST chalcogenide to reversibly tune the resonant frequency of the nanoantenna array in IR spectrum by applying a femtosecond Ti:sapphire laser pulse [85]. Also, Feldmann et al. applied pJ pulses to reset (re-amorphized) rectangular waveguide arrays with GST chalcogenide crossing points which perform arithmetic operations to level 0 [86]. We have demonstrated one working device which we could revert the ChG to its initial amorphous state by applying high voltage [87]. Reverting ChG to its initial 
amorphous condition, allows multiple time use of the device [74,88]. The total number of covalent bonds do not change during crystallization, as a result of which the total energy of the system does not essentially change and all initial properties of ChG can be restored. This indicates the comparable stability of the two phases and the possibility for their interchange. This is a warranty for the performance stability of the devices subject of this study and their reversibility.

B- Chalcogenide glasses are radiation hardened: The lack of order in the chalcogenide glasses and the presence of lone pair p-shell electrons are the reasons behind the radiation hardness and the unique electrical and optical properties of these materials. They have band tail states and localized states which pin the Fermi level independently of surrounding radiation in the middle of the bandgap. ChGs contain a high number of defects $[89,90]$. These defects combined with the defects occurring as a result of irradiation [91] become populated in very close proximity due to which their recombination occurs fast and the properties of the material remain quite stable and radiation hardened. 


\section{CHAPTER 3:}

\section{OPTICAL FIBER BASED TEMPERATURE SENSOR MODELING}

This chapter provides details on the modeling of optical fiber based temperature sensors. Six different in-house ChGs-capped optical fibers were simulated and the reflected power from interface of optical fiber and ChG in two amorphous and crystalline phases of ChG were obtained. By covering the tip of the optical fiber with ChG, we constructed a novel and reversible fiber-optic temperature sensor that measures the Fresnel reflection. The intensity of the Fresnel reflection is proportional to the absorption coefficient of ChG. The simulation results demonstrate the performance of the sensors in two different phases of $\mathrm{ChG}$ and verifies the concept. Before simulation, we need to study optical properties of each of the synthesized ChG. Then, we insert these values to the simulation and study their responses in two phases separately.

\subsection{Determination of the Optical Constants of}

\section{In-house Synthesized ChGs}

The optical properties of each of the in-house synthesized ChGs were measured using the following method. First, on a silicon substrate, a thin film of the composition under study was thermally deposited. Next, ellipsometry (J. A. Woollam's M-2000) with 
in-situ heating using an enclosed ellipsometer-compatible hot stage (THMSEL600 by Linkam Scientific) was performed to obtain the refractive indices $(n)$ and extinction coefficients $(k)$ from the film by applying the Cauchy dispersion model using the system's software (CompleteEASE). The index was measured at several wavelengths from 630.7-1560 nm to obtain a dispersion curve. An ellipsometer consisting of a light source, a polarizer, optical compensators, an analyzer, and a detector, as seen in Figure. 3.1 was used. The angle of incidence is equal to the angle of reflection.

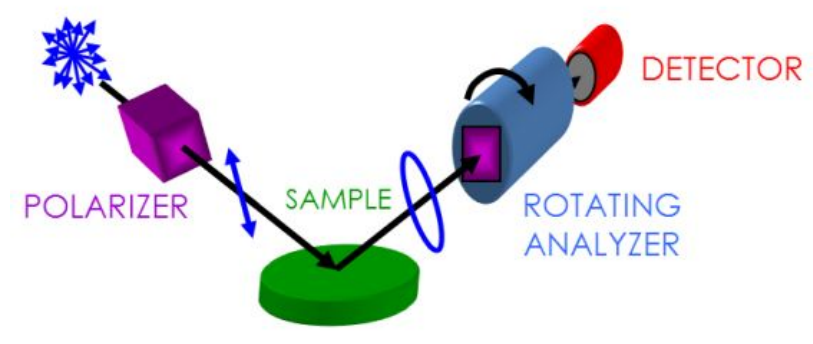

Figure 3.1: Illustration of the setup of an ellipsometry measurement [92].

The working mechanism of ellipsometer is based on changes in light polarization after it is reflected off the material surface. This polarized light can be defined by its $p$ (parallel) and $s$ (perpendicular) components. The detector measures the amplitude of reflected light, and then normalizes to the initial values. These values are the complex reflection coefficients and can be denoted as $r_{p}$ and $r_{s}$. The ratio of the complex reflection coefficients, $\rho$, is calculated using equation 3.1 shown below:

$$
\rho=\frac{r_{p}}{r_{s}}=\tan (\Psi) e^{i \Delta}
$$

where $\Delta$ and $\tan (\Psi)$ are the phase shift and the ratio of amplitude change, respectively, of the $p$ and $s$ components after reflection. An optical model, such as the 
Cauchy dispersion law (Equation. 3.2), can be applied to determine the dispersion curves and the thickness of the film after these measurements have been made at multiple wavelengths. Knowing the thickness of the film helps in accuracy of the optical contact measurement. The reason is that the refractive index determines the light wave velocity and refracted angle and the film thickness which has an effect on path length of light traveling through the film. These two parameters contribute to the delay between surface reflection and light traveling through the film [92]. For transparent materials, the index is described using Cauchy equation given as:

$$
n=A+\frac{B}{\lambda^{2}}+\frac{C}{\lambda^{4}}
$$

Where $n$ is the refractive index, $\lambda$ is the wavelength and $A, B, C$ are coefficients that can be determined for a material by fitting the equation to measured refractive indices at known wavelengths. The measured complex refractive indices of in-house synthesized Ge-Se and Ge-S compositions in amorphous and crystalline phases (Table 3.1) from [93] are imported into the simulation models. Table 3.1 summarizes the $T_{\mathrm{g}}, T_{\mathrm{o}}$ and $T_{\mathrm{c}}$ for all synthesized and studied compositions. Among the big ChG family, Ge-Se and Ge-S systems were chosen in the proposed device because - (1) these compositions are thermally stable within the range $400{ }^{\circ} \mathrm{C}-650{ }^{\circ} \mathrm{C}$ and $(2)$ the glass forming regions of these systems are quite extensive, assuring a significant number of compositions to work with. An example of the measured refractive index $(n)$ and extinction coefficient $(k)$ for a $\mathrm{Ge}_{40} \mathrm{Se}_{60}$ composition as a function of temperature at $1550 \mathrm{~nm}$ wavelength is shown in Table 3.2.

It can be seen that the refractive index $(n)$ and extinction coefficient $(k)$ are very different in the crystalline and the amorphous phases. Among them, three types of 
Table 3.1: Summary of the measured complex refractive indices of synthesized glasses in amorphous and crystalline phases at $1550 \mathrm{~nm}$ wavelength.

\begin{tabular}{cccccc}
\hline \multirow{2}{*}{ Composition } & \multicolumn{2}{c}{ Refractive index } & \multicolumn{3}{c}{ Temperature $\left({ }^{\circ} \mathrm{C}\right)$} \\
\cline { 2 - 6 } & Amorphous & Crystalline & $\mathrm{T}_{\mathrm{g}}$ & $\mathrm{T}_{\mathrm{o}}$ & $\mathrm{T} \mathrm{c}$ \\
\hline $\mathrm{Ge}_{30} \mathrm{~S}_{70}$ & $2.17406+\mathrm{i} 0$ & $1.77269+\mathrm{i} 0.11865$ & 402 & 572 & 605 \\
\hline \multirow{2}{*}{$\mathrm{Ge}_{40} \mathrm{~S}_{60}$} & $2.6768+\mathrm{i} 0$ & $2.72309+\mathrm{i} 0.17664$ & 355 & 408 & 413 \\
& & & & 480 & 489 \\
\hline $\mathrm{Ge}_{33} \mathrm{~S}_{67}$ & $2.31779+\mathrm{i} 8.28 \mathrm{e}-6$ & $1.92455+\mathrm{i} 0.02458$ & 435 & 644 & 694 \\
\hline $\mathrm{Ge}_{30} \mathrm{Se}_{70}$ & $2.37646+\mathrm{i} 4.06 \mathrm{e}-5$ & $3.12455+\mathrm{i} 0.25837$ & 334.8 & 440.9 & 470.4 \\
\hline $\mathrm{Ge}_{40} \mathrm{Se}_{60}$ & $2.63104+\mathrm{i} 0.00575$ & $3.1099+\mathrm{i} 0.211$ & 343.7 & 446.6 & 472.3 \\
\hline $\mathrm{Ge}_{33} \mathrm{Se}_{67}$ & $2.38753+\mathrm{i} 0.00402$ & $2.30756+\mathrm{i} 0.02011$ & 396.3 & 485.4 & 527.7 \\
\hline
\end{tabular}

compositions have been chosen for detailed characterization - chalcogen rich glasses containing 30 at.\% Ge, stoichiometric compositions ( $\left.\mathrm{GeSe}(\mathrm{S})_{2}\right)$ with 33 at.\% Ge and Ge rich compositions containing 40 at.\% Ge. These are the most characteristic representatives of these two systems, whose structure, based on the valence requirements of the participating atoms, is characterized with Ge-Chalcogen tetrahedra in which the Ge atom is in the center of the tetrahedron surrounded by 4 chalcogen atoms [94]. These tetrahedra can be connected to each other at their corners, forming cornersharing structural units, or along their edges, forming edge-sharing units [95]. The other member of this structure are chalcogenide chains in which the chalcogen elements are two-fold coordinated. The Se-Se chains in the Ge-Se system are usually situated within the tetrahedral structure and connect the tetrahedral structural units, while in the Ge-S system the S-S units they usually form 8-member rings. Ge-S systems are phase separated from the main tetrahedral structure. Chalcogen chain structures are characteristic for the chalcogen rich compositions and they are not supposed to occur in the stoichiometric compositions. However, due to the disordered character of the glass structure, they are available in some cases and are usually 
Table 3.2: Measured complex refractive index of $\mathrm{Ge}_{40} \mathrm{Se}_{60}$ at different temperatures at $1550 \mathrm{~nm}$ wavelength.

\begin{tabular}{cccc}
\hline Temperature $\left({ }^{\circ} \mathrm{C}\right)$ & Refractive index & Temperature $\left({ }^{\circ} \mathrm{C}\right)$ & Refractive index \\
\hline 25 & $2.717+\mathrm{i} 0.00547$ & 400 & $2.70088+\mathrm{i} 0.01636$ \\
\hline 100 & $2.171516+\mathrm{i} 0.00575$ & 450 & $3.35909+\mathrm{i} 0.25735$ \\
\hline 150 & $2.695050+\mathrm{i} 0.00547$ & 472 & $3.29764+\mathrm{i} 0.09341$ \\
\hline 200 & $2.66978+\mathrm{i} 0.00513$ & 479 & $3.1099+\mathrm{i} 0.2211$ \\
\hline 250 & $2.63104+\mathrm{i} 0.00575$ & 484 & $3.14107+\mathrm{i} 0.22572$ \\
\hline 300 & $2.59792+\mathrm{i} 0.00563$ & 500 & $3.2688+\mathrm{i} 0.21606$ \\
\hline 350 & $2.70057+\mathrm{i} 0.00938$ & & \\
\hline
\end{tabular}

considered as "wrong $\mathrm{Se}(\mathrm{S})$ - Se(S) bonds". This respectively leads through lack of chalcogens to formation of "wrong" - Ge-Ge bonds [96]. Indeed, the number of the chalcogen-chalcogen and Ge-Ge wrong bonds are the same, which corresponds to the broken chemical order in the glasses. In the Ge rich compositions the Ge-chalcogen tetrahedra forms the structure, similar to that of ethane, which is just called ethanelike. In this structure, two Ge atoms are connected to each other and each has three more chalcogen neighbors for satisfying the Ge atoms valence requirements [97].

\subsubsection{Fiber Sensor Modeling}

A schematic of the model we used for simulating the sensor characteristics is shown in Figure. 3.2 [98]. The chalcogenide thin film can be deposited directly over the tip of the fiber covering the fiber core. Either ink-jet printing and thermally evaporated methods can be used to print the two electrodes on the ChG film, as shown in Figure. 3.3. Wire bonding can be utilized to make contacts to the $\mathrm{ChG}$ film. The outer gold capillary sections serve as electrodes, as well as to provide operability in high temperature and radiation environments. Once the entire sensor is at room temperature, Joule heating achieved by application of electrical field, will reverse the ChG material's crystalline phase into amorphous phase again, through melting the crystal- 


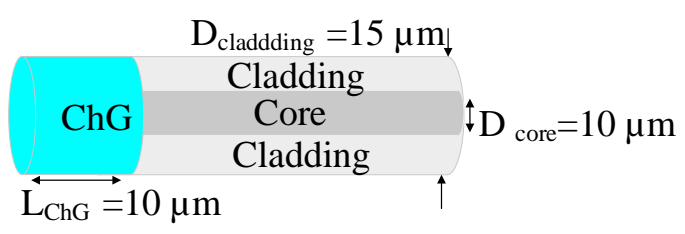

Figure 3.2: Schematic cross-section of proposed ChG-capped optical fiber tipbased temperature sensor.

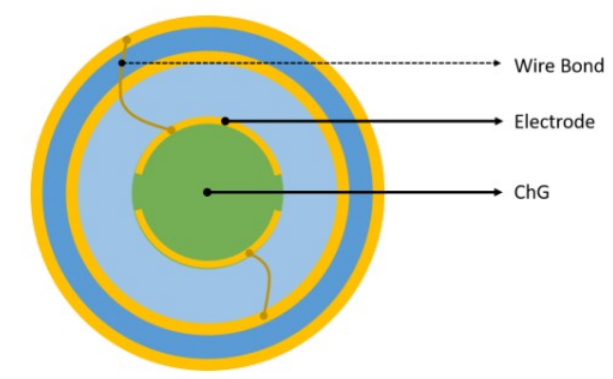

Figure 3.3: Schematic cross-section of proposed ChG-capped optical fiber tipbased temperature sensor.

lized $\mathrm{ChG}$ and the device can be reused. To this end, we have simulated the proposed design. The design is modeled using PhotonDesign software modules (FIMMWAVE and FIMMPROP), which utilize a fully vectorial finite-difference method. The optical properties of single mode pure silica Rad Hard optical fiber (Nufern: S1550-HTA) with core refractive index of 1.45735 and cladding refractive index of 1.44715 at 1550 $\mathrm{nm}$ wavelength are used to represent the fiber. In the amorphous phase, ChG behaves like a dielectric material with a lower absorption coefficient compared to that in the crystalline phase. From Table 3.1, it is evident that compared to the amorphous phase, the complex refractive index of $\mathrm{ChG}$ in the crystalline phase is vastly different. Thus, the intensity of the reflected light back into the fiber from the ChG-fiber tip interface is also expected to be different. This forms the basis of our sensor's operating mechanism. This fiber optic sensor based on Fresnel reflection measures the 
reflected intensities from the fiber end facet. Figure. 3.4 shows the Fresnel reflection phenomenon occurring at the fiber end-ChG interface.
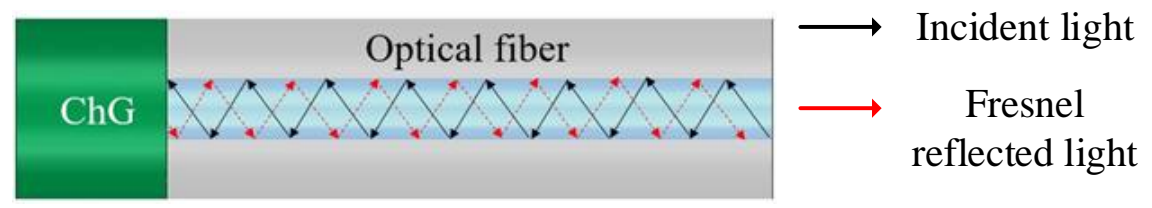

Figure 3.4: fiber-optic sensor based on Fresnel reflection.

The reflection coefficients of parallel and perpendicular polarization are respectively given by

$$
\begin{array}{r}
r_{n}=\frac{n_{\text {core }} \cos \theta_{1}-n_{C h G} \cos \theta_{2}}{n_{\text {core }} \cos \theta_{1}+n_{C h G} \cos \theta_{2}} \\
r_{p}=\frac{n_{C h G} \cos \theta_{1}-n_{\text {core }} \cos \theta_{2}}{n_{C h G} \cos \theta_{1}+n_{\text {core }} \cos \theta_{2}}
\end{array}
$$

where $n_{C h G}$ and $n_{\text {core }}$ are the refractive indices of the optical fiber and the material surrounding the fiber end surface, respectively, and $\theta_{1}$ and $\theta_{2}$ are the angles of the incidence and reflection, respectively. In single mode fibers, the reflection coefficient can be simplified by the paraxial assumption $\left(\theta_{1} \approx \theta_{2} \approx 0\right)$ :

$$
R=\left|r_{p}\right|^{2}=\left|r_{n}\right|^{2}=\left|\frac{n_{\text {core }}-n_{C h G}}{n_{\text {core }}+n_{C h G}}\right|^{2}
$$

In this sensor, the Fresnel reflection intensity is a function of the refractive indices of the optical fiber and covering ChG. Owing to the different refractive index in two different ChG phases, the reflection intensity varies with temperature. We have simulated the transmitted and reflected powers in 6 in-house synthesized $\mathrm{ChG}\left(\mathrm{Ge}_{30} \mathrm{~S}_{70}\right.$, 
$\left.\mathrm{Ge}_{40} \mathrm{~S}_{60}, \mathrm{Ge}_{33} \mathrm{~S}_{67}, \mathrm{Ge}_{30} \mathrm{Se}_{70}, \mathrm{Ge}_{40} \mathrm{Se}_{60}, \mathrm{Ge}_{33} \mathrm{Se}_{67}\right)$ capped fiber devices in both amorphous phase and crystalline phases. The simulated results are shown in Figure. 3.5 through Figure. 3.10.

For example, the simulated result in Figure. 3.5(a,c) shows the transmitted power as a function of ChG material cap thickness in $\mathrm{Ge}_{40} \mathrm{Se}_{60}$ capped fiber device in the amorphous phase. It can be seen that due to a low absorption coefficient and a modest difference in the refractive index between $\mathrm{ChG}$ and fiber mode, most of the light transmits through the ChG. When the temperature is higher than the crystallization temperature of $\mathrm{ChG}\left(T>T_{\mathrm{c}}\right)$, the $\mathrm{ChG}$ material crystallizes. In this phase, the $\mathrm{Ge}_{40} \mathrm{Se}_{60}$ has higher refractive index, as well as a higher extinction ratio coefficient (behaves like a metal). Thus, a higher fraction of light is reflected back into the fiber, and the transmitted power inside the $\mathrm{ChG}$ decays rapidly into the material, as shown in Figure. 3.5(b,d). This change in the reflected power level occurs at very welldefined temperatures $\left(\sim T=T_{\mathrm{c}}\right)$, monitoring of which provides information regarding the node temperature. 


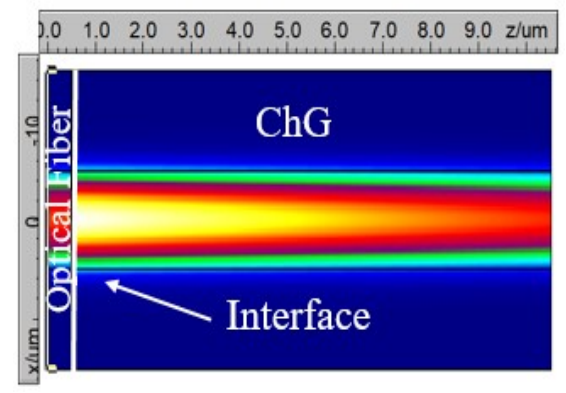

(a)

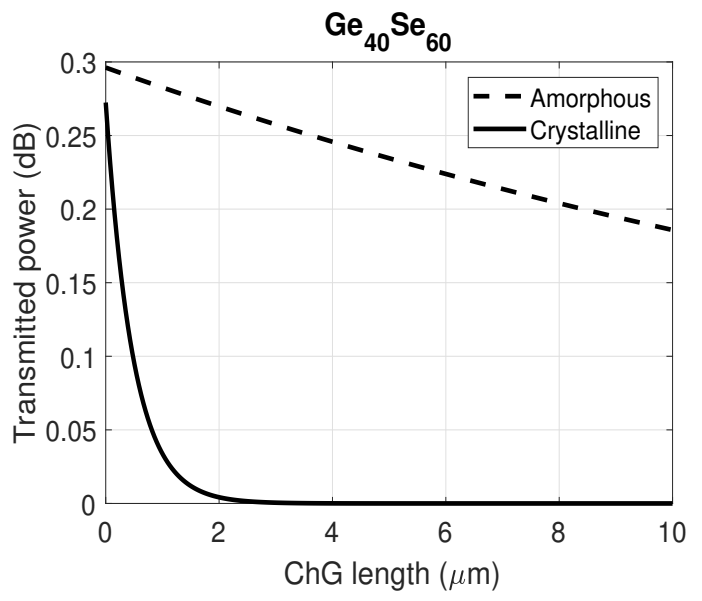

(c)

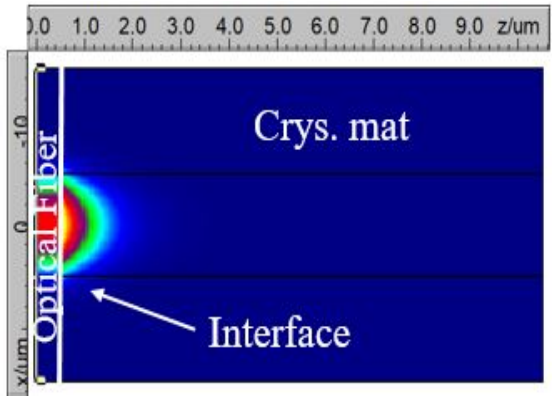

(b)

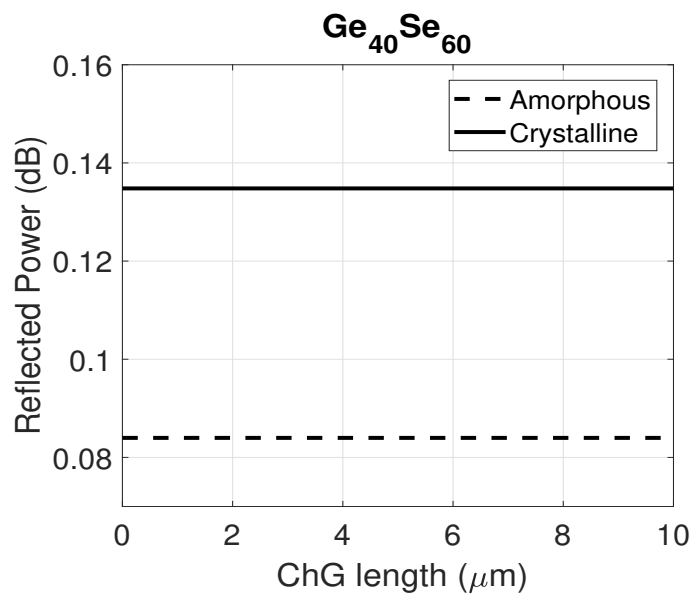

(d)

Figure 3.5: $\mathrm{Ge}_{40} \mathrm{Se}_{60}$ capped fiber device: Power distribution at the fiber ChG interface for: a) transmitted power in amorphous phase, b) transmitted power in crystalline phase, c) transmitted power as a function of the length of ChG, d) reflected power as a function of the length of ChG. Solid curve indicates crystalline phase and the dashed-dotted curve indicates amorphous phase. 


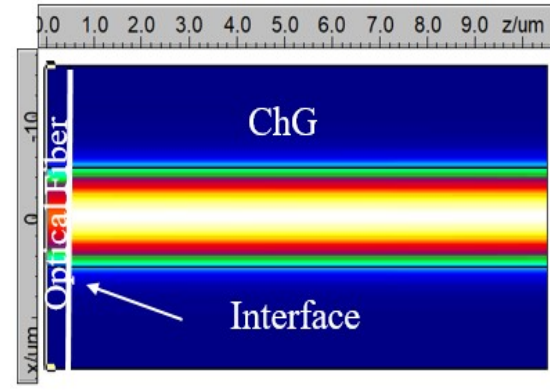

(a)

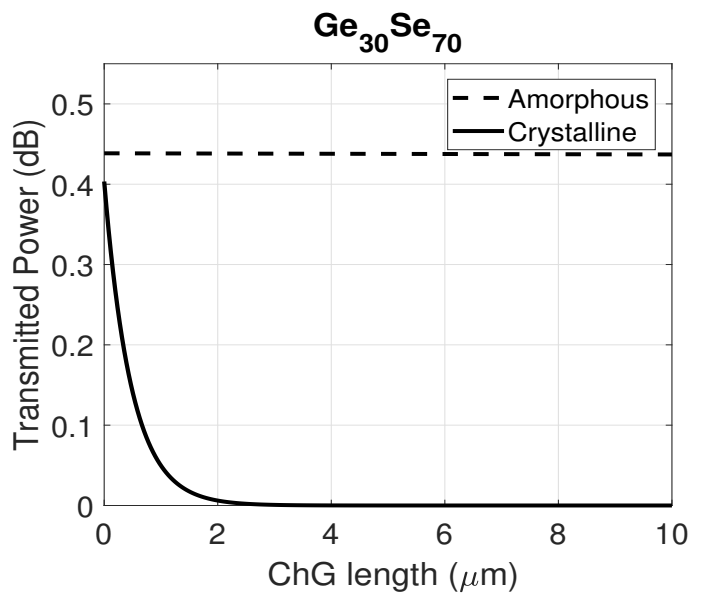

(c)

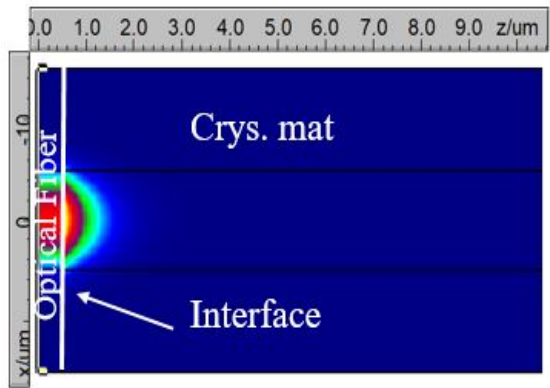

(b)

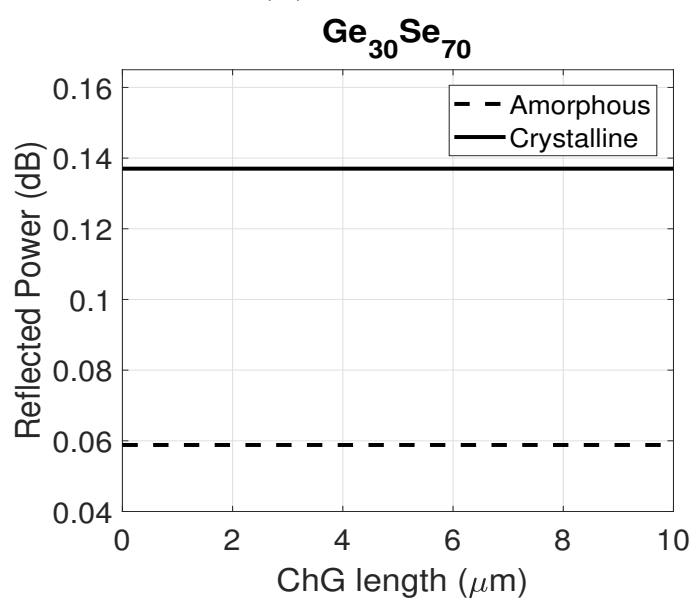

(d)

Figure 3.6: $\mathrm{Ge}_{30} \mathrm{Se}_{70}$ capped fiber device: Power distribution at the fiber ChG interface for: a) transmitted power in amorphous phase, b) transmitted power in crystalline phase, c) transmitted power as a function of the length of ChG, d) reflected power as a function of the length of ChG. Solid curve indicates crystalline phase and the dashed-dotted curve indicates amorphous phase. 


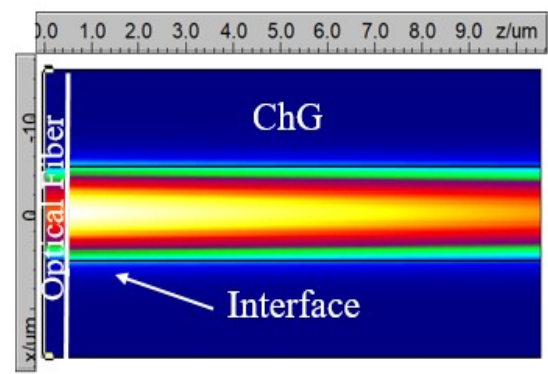

(a)

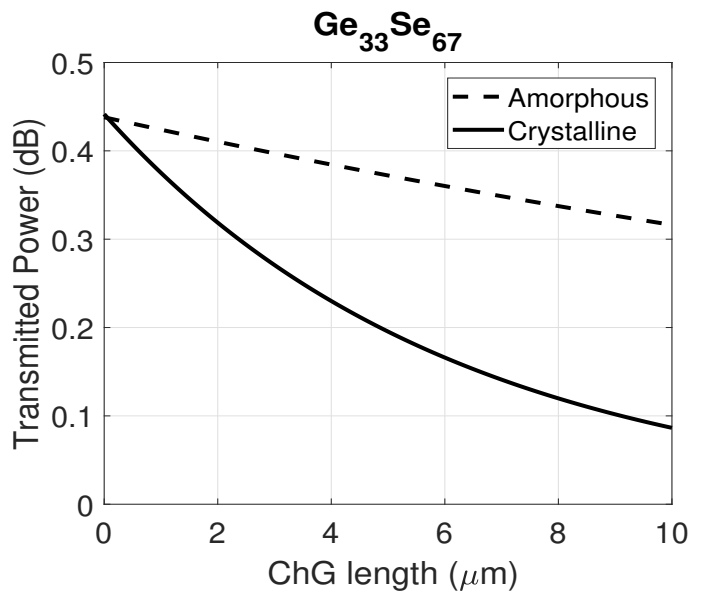

(c)

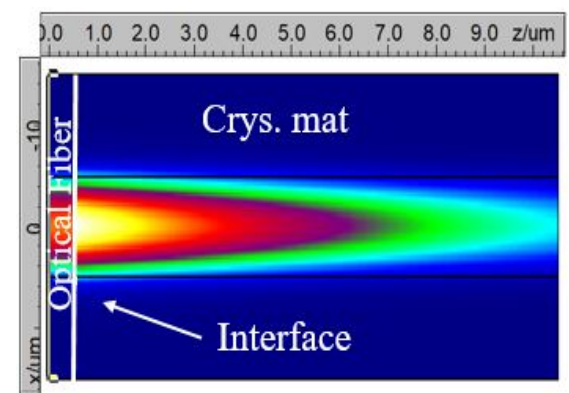

(b)

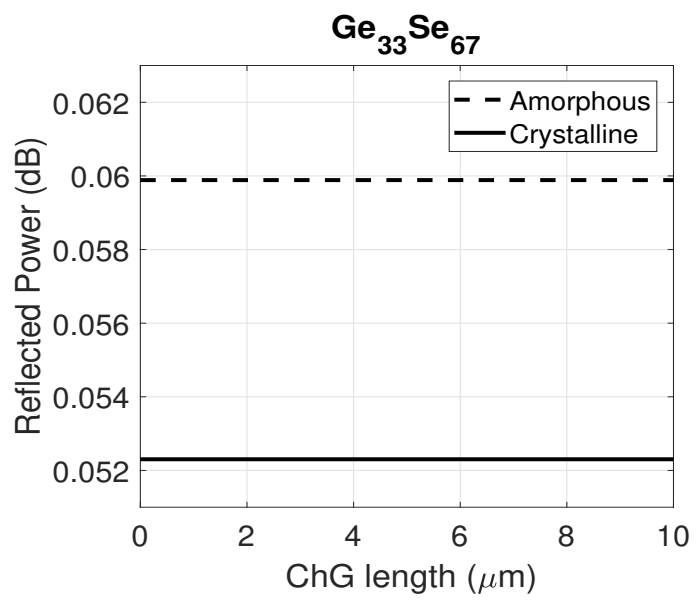

(d)

Figure 3.7: $\mathrm{Ge}_{33} \mathrm{Se}_{67}$ capped fiber device: Power distribution at the fiber ChG interface for: a) transmitted power in amorphous phase, b) transmitted power in crystalline phase, c) transmitted power as a function of the length of ChG, d) reflected power as a function of the length of ChG. Solid curve indicates crystalline phase and the dashed-dotted curve indicates amorphous phase. 


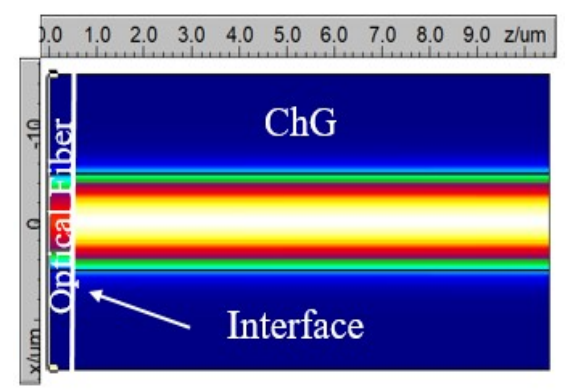

(a)

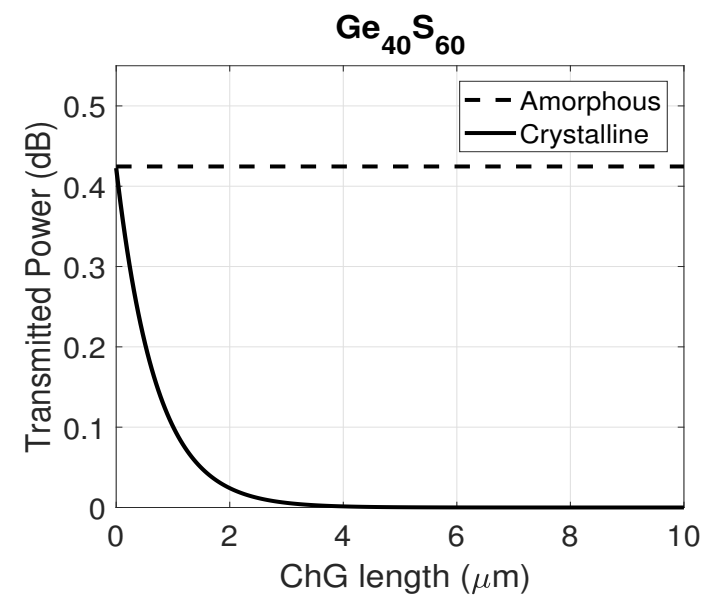

(c)

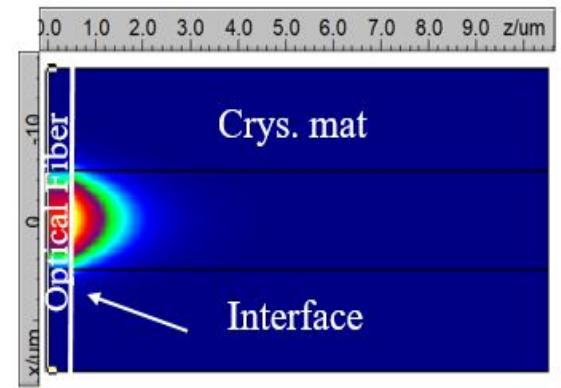

(b)

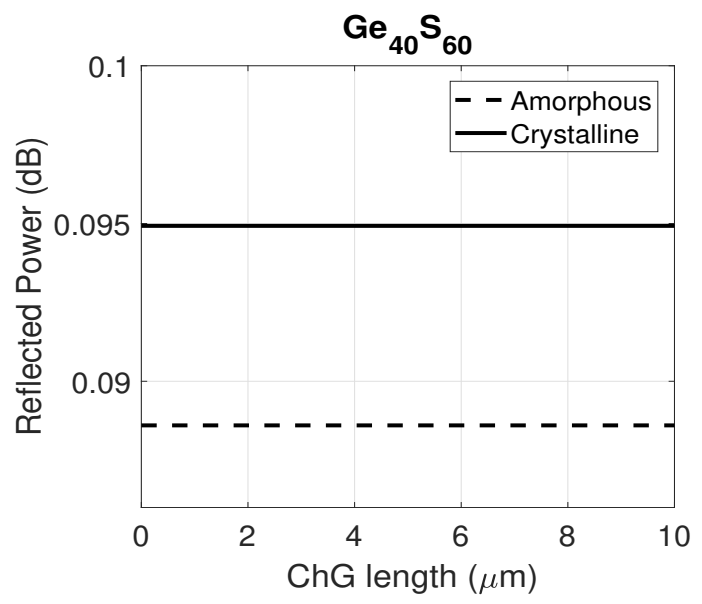

(d)

Figure 3.8: $\mathrm{Ge}_{40} \mathrm{~S}_{60}$ capped fiber device: Power distribution at the fiber ChG interface for: a) transmitted power in amorphous phase, b) transmitted power in crystalline phase, c) transmitted power as a function of the length of ChG, d) reflected power as a function of the length of ChG. Solid curve indicates crystalline phase and the dashed-dotted curve indicates amorphous phase.

In Figure. 3.5d the reflected power into the fiber shows different power levels in the crystalline phase and amorphous phases due to the different extinction coefficient of the material. Similarly, the effect of all the other ChG composition's refractive index profiles in their amorphous and crystalline phases on the reflected power level can be explained. For most of the studied compositions, selecting an end cap thickness less than $10 \mu \mathrm{m}$ provided a relative high extinction ratio that can be easily detected. From the simulation results, it is observed that all compositions except $\mathrm{Ge}_{33} \mathrm{~S}_{67}, \mathrm{Ge}_{33} \mathrm{Se}_{67}$, 


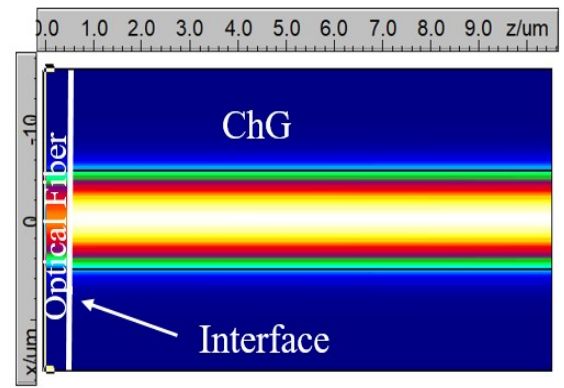

(a)

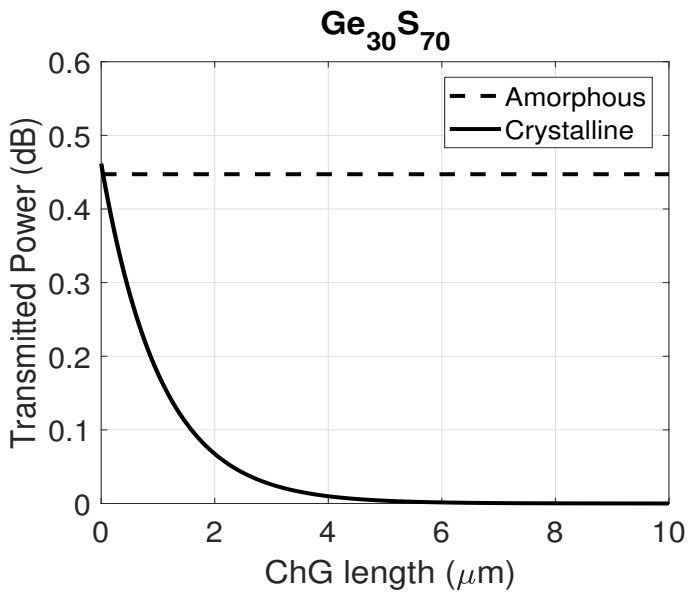

(c)

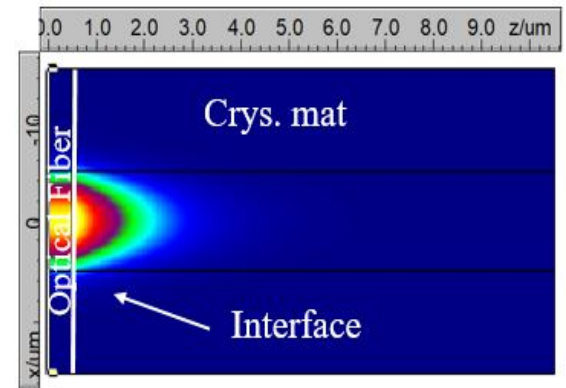

(b)

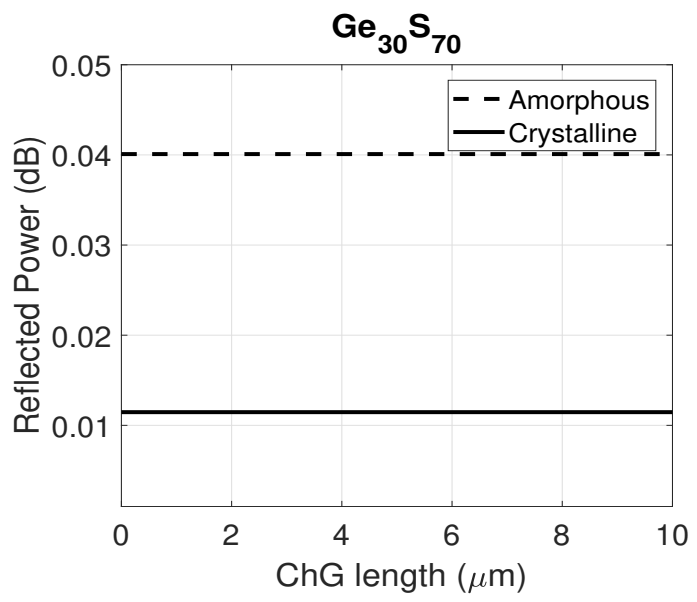

(d)

Figure 3.9: $\mathrm{Ge}_{30} \mathrm{~S}_{70}$ capped fiber device: Power distribution at the fiber ChG interface for: a) transmitted power in amorphous phase, b) transmitted power in crystalline phase, c) transmitted power as a function of the length of ChG, d) reflected power as a function of the length of ChG. Solid curve indicates crystalline phase and the dashed-dotted curve indicates amorphous phase.

and $\mathrm{Ge}_{30} \mathrm{~S}_{70}$ show a considerable difference in extinction coefficient; examples are $\mathrm{Ge}_{40} \mathrm{~S}_{60}, \mathrm{Ge}_{40} \mathrm{Se}_{60}$ and $\mathrm{Ge}_{30} \mathrm{Se}_{70}$. All compositions (except $\mathrm{Ge}_{33} \mathrm{~S}_{67}, \mathrm{Ge}_{33} \mathrm{Se}_{67}$ ) crystallize by a heterogeneous process by which several different crystalline phases appear and the structure becomes much denser, which leads to an increase in the refractive index [93]. The stoichiometric compositions, $\mathrm{Ge}_{33} \mathrm{~S}_{67}$ and $\mathrm{Ge}_{33} \mathrm{Se}_{67}$ have similar total reflected power in both phases, which indicates that small changes occur in the material structure and band gap during the crystallization process, which is homogeneous 
in its nature [93].

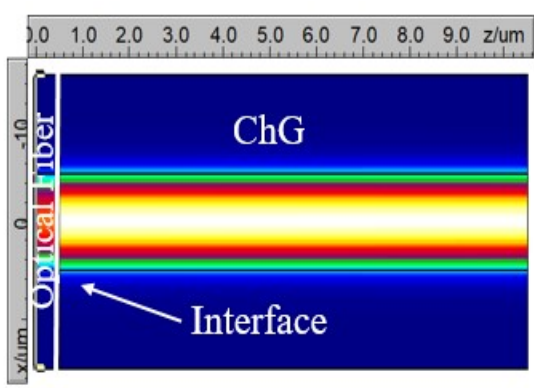

(a)

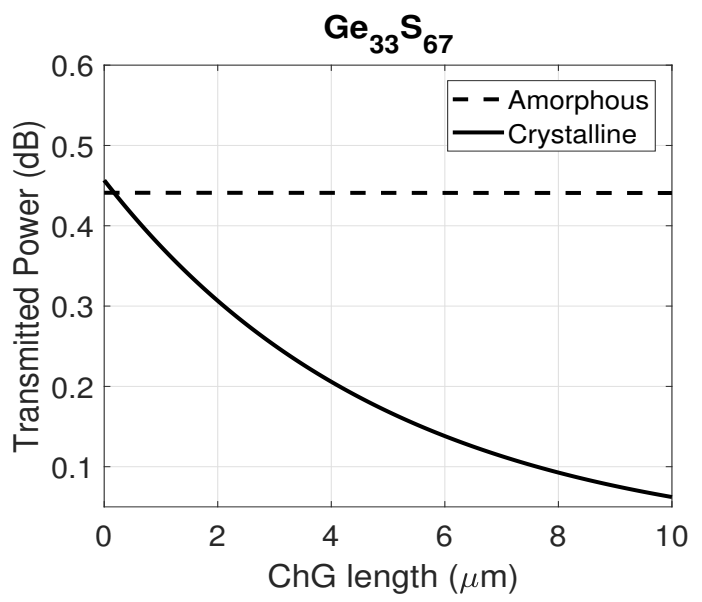

(c)

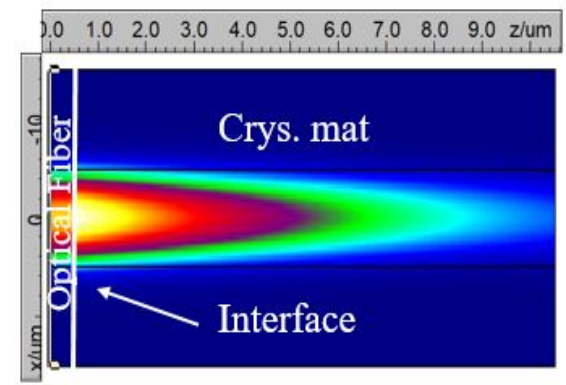

(b)

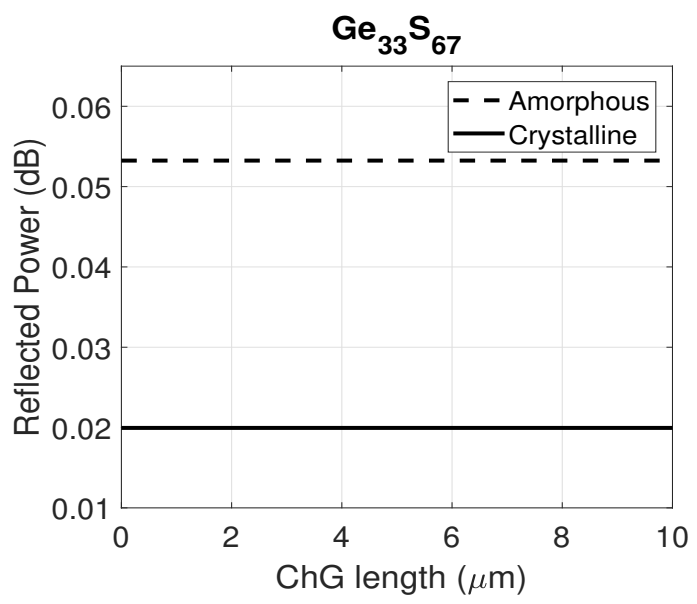

(d)

Figure 3.10: $\mathrm{Ge}_{33} \mathrm{~S}_{67}$ capped fiber device: Power distribution at the fiber ChG interface for: a) transmitted power in amorphous phase, b) transmitted power in crystalline phase, c) transmitted power as a function of the length of ChG, d) reflected power as a function of the length of ChG. Solid curve indicates crystalline phase and the dashed-dotted curve indicates amorphous phase.

We have summarized the simulated reflected power as a function of ChG length by increasing Ge atom in Figure. 3.11. Figure. 3.11a shows the normalized reflected power back into the fiber for Ge-S (left) and Ge-Se (right) capped fiber devices, respectively, as a function of $\mathrm{ChG}$ cap thickness. The reflected power into the fiber shows different power levels in the crystalline phase and amorphous phases due to the different extinction coefficient of the material, as shown in Figure. 3.11b. 

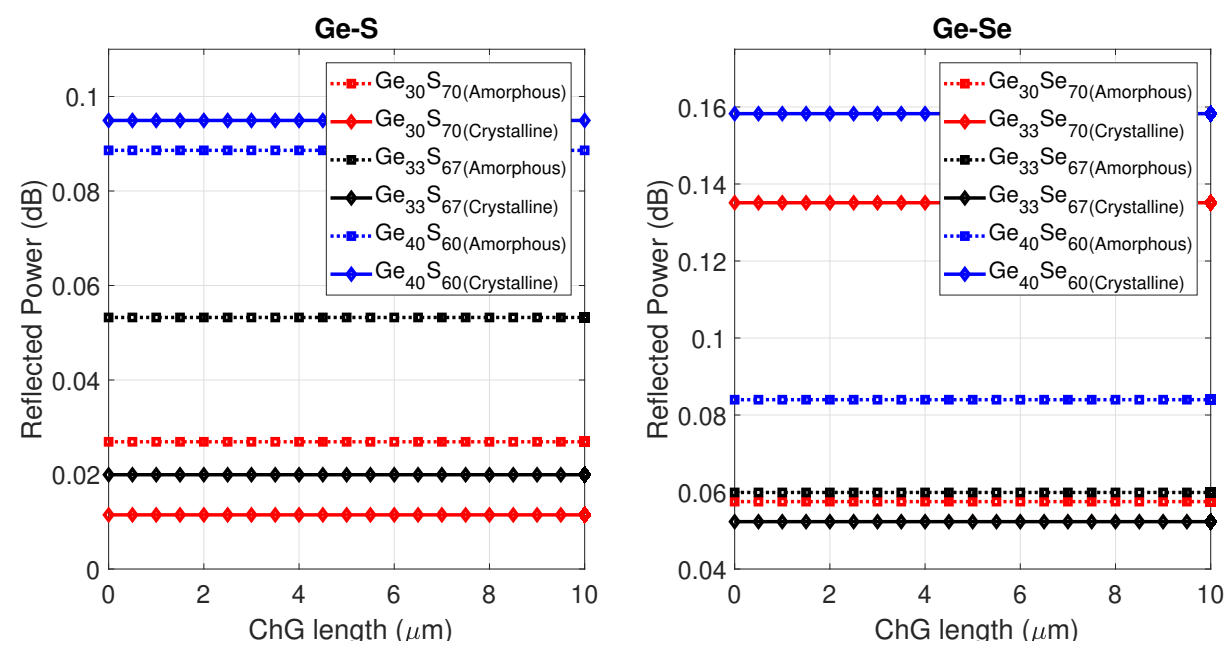

(a)

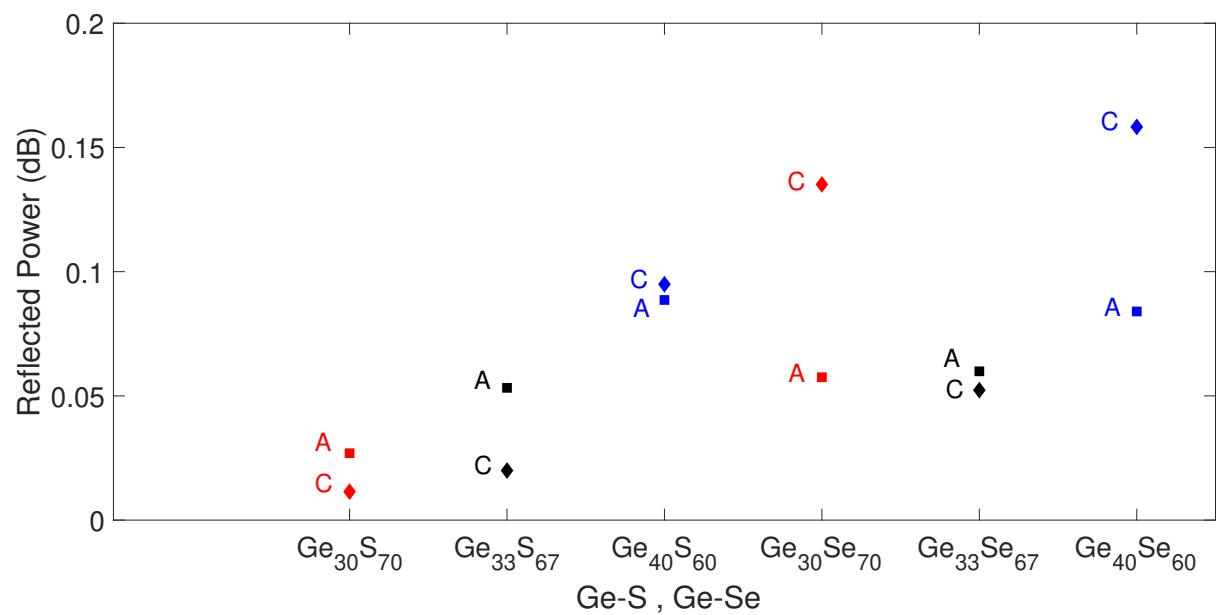

(b)

Figure 3.11: a) Normalized reflected power of the fibers capped with in-house synthesized Ge-S (top row) and Ge-Se (bottom row) compositions. Solid curve indicates crystalline phase and the dashed-dotted curve indicates amorphous phase, b) Normalized reflected power of Ge-Se and Ge-S coated fiber tips in amorphous and crystalline phases.

\subsection{Summary}

In this chapter, we looked at different in-house ChGs covering the top surface of the waveguide simulation. The measured optical properties of the synthesized Ge-S and 
Ge-Se ChGs at Boise State University is reported. Using these values, we modeled the optical fiber based temperature sensors. The designed fiber optic sensors with ChG capped end facets show higher reflectivity in crystalline phase compared to amorphous phase of the ChG. In the next chapter, we discuss the fabrication procedure of optical fiber based temperature sensor. 


\section{CHAPTER 4:}

\section{FABRICATION AND CHARACTERIZATION OF OPTICAL FIBER BASED TEMPERATURE SENSORS}

This chapter demonstrates the optical fiber based temperature sensor fabrication process. First, the preparation of the optical fiber tip is discussed. Next, we describe two methods to cover the fiber tip with in-house synthesized ChGs. Then, the testing set up to measure the temperature response of the proposed sensor is presented. Finally, we derive a method to analyze the obtained measurement results to confirm the functionality of the proposed sensor.

\subsection{Optical Fiber Tip Coating}

Rad Hard fibers are a crucial component in the design of the temperature sensing system since they determine how light is fed to the sensing element, and how data is transmitted back to the control facility. Traditional glass fibers are highly sensitive to radiation, and radiation exposure causes darkening and induces additional loss over prolonged use [99]. Therefore, to fabricate proposed optical fiber based temperature sensors, we used Rad Hard fibers from a local fiber manufacturer, Fiberguide Industries. Fiberguide specializes in Rad Hard gold coated fibers that provides fiber pro- 
tection up to $700{ }^{\circ} \mathrm{C}$. A schematic of the gold-coated Rad Hard fiber from Fiberguide Industries is shown in Figure. 4.1. The high temperature performance, combined with excellent corrosion resistance, makes it an ideal fiber for many high temperature applications, such as in-pile, turbine flame detection, oil and gas down-hole sensing, and high vacuum or pressure applications.

Our proposed sensor is fabricated using radiation hardened single-mode pure fused silica core gold-coated fiber (FiberGuide AFS50/125/155G). The length of the employed fiber is about $50 \mathrm{~cm}$. This length allows us to handle the fiber carefully. The fabrication process involves two steps. In the first step, to deposit ChG on the tip of optical fiber, we need to cleave the tip before starting the process. As the cleaving tools we have cannot cleave the gold coated fiber, we stripped about 3-4 $\mathrm{cm}$ of the gold coat first by immersing the tip in Aqua Regia solution, which is a combination of hydrochloric acid and Nitric acid as shown in Figure. 4.2. The optical fiber is dipped in the solution for 5-10 minutes. Figure. 4.3 shows a microscope image of the gold stripped portion of the fiber.

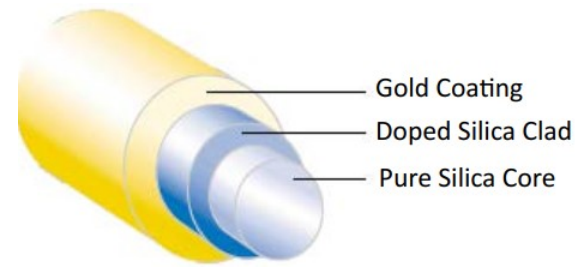

Figure 4.1: Gold coated fiber [100].

In the second step, the exposed fiber tip was cleaved using a standard fiber cleaver and the cleaved tip was coated with ChG. The effects of two coating methods to fabricate the sensors were studied - a) dip coating, and b) thermal evaporation. While dip coating relies on forming a ChG solution, the thermal evaporation method is a 


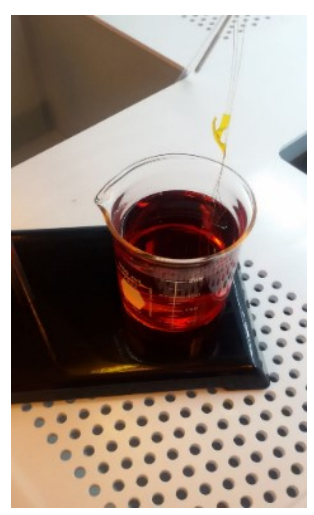

(a)

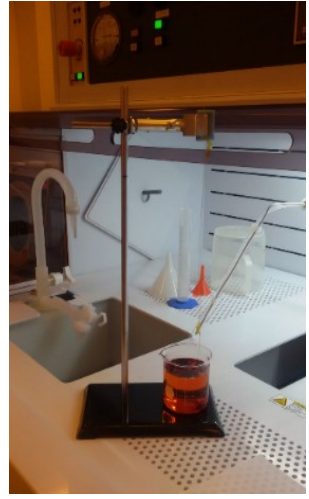

(b)

Figure 4.2: Aqua Regia solution, b) gold coated fiber is dipped in solution.

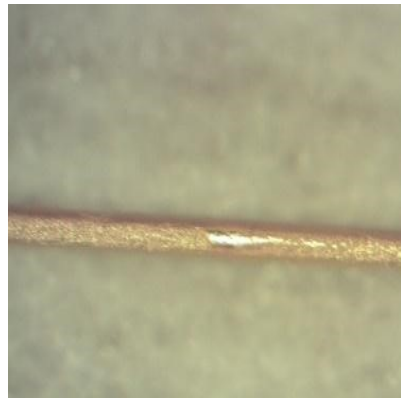

(a)

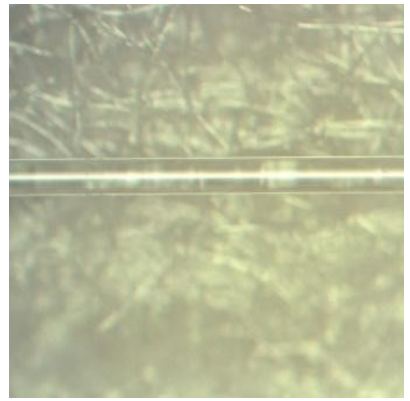

(b)

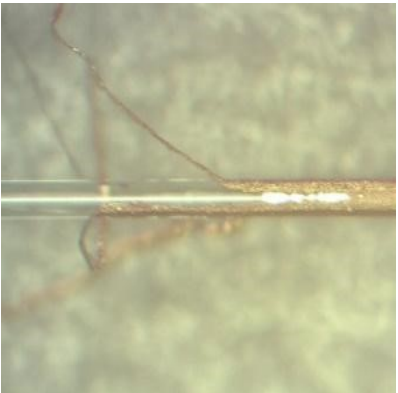

(c)

Figure 4.3: a) Gold coated fiber under microscope, b) etching after 600 seconds, c) Transition part between etch and gold.

standard process and provides a baseline for comparison. The fabrication process is as follows:

\subsubsection{Dip Coating}

Dip coating consists: (a) of immersing a substrate (exposed fiber tip) into a bath containing coating material at a constant speed, preferably judder free, leaving the substrate fully immersed and motionless to allow for coating material to apply itself to the substrate, (b) then withdrawal of the substrate from fluid solution bath at a constant speed to avoid any judders, gravitational draining and solvent evap- 


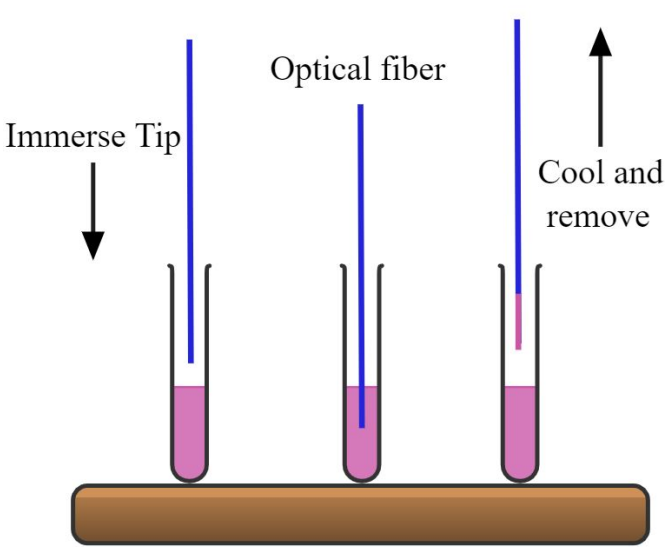

Figure 4.4: Fiber coating method by immersion of the fiber into ink or melt of ChG.

oration resulting in deposition of a solid film [101]. The coated piece can then be dried by force-drying or baking. This method, compared to other techniques, such as chemical vapor deposition and, sputtering is straightforward, less expensive, and requires minimal setup costs. There are several parameters that can affect the film thickness, such as coating viscosity, withdrawal under variable angle of inclination, angle between substrate and the liquid surface, and rate of withdrawal from the bath. Particle size, reactivity, relative rate of condensation and evaporation, liquid surface tension, and dipping speed varies the resulting structure of depositing film [102,103]. A non-uniform film thickness deposited on the substrate is expected in this method.

One technique to fabricate the proposed sensor devices is by briefly immersing the cleaved fiber tip in a ChG nanoparticle ink bath, as shown in Figure 4.4 [104, 105]. ChGs rapidly concentrate on the fiber surface by gravitational draining and evaporation often accompanied by continued condensation reactions [101]. The solvent from the solution on the fiber evaporates as the fiber is drawn. Once the fiber was extracted, the coatings were left to dry for 24 hours at room temperature. Then, the 


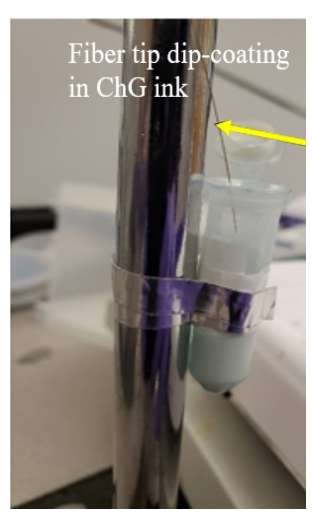

(a)

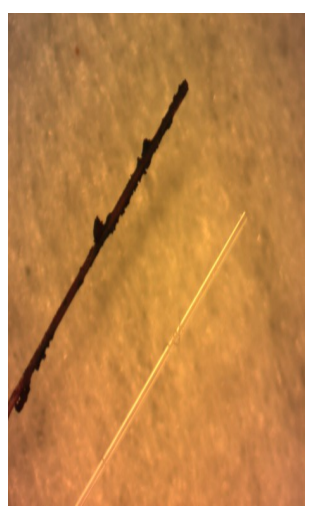

(b)

Figure 4.5: Dip-coating fabrication method. a) experiment setup to coat fiber tip with chalcogenide glass, b) an ink coated fiber tip (dark) and a blank fiber tip (transparent).

coatings were further cured using a hot chuck in a two-step process: (1) the coated fiber was heated at $100{ }^{\circ} \mathrm{C}$ for 2 hours to slowly dry the solvent, cyclohexanone, without creating cracks in the film, and (2) the fiber tip was placed on a hot plate and heated at $350{ }^{\circ} \mathrm{C}$ for 15 minutes to decompose the surfactants in the ink, Ethyl Cellulose. Once cooled, the fiber tip was dip-coated with protective spin-on-glass layer for isolation of the sensor from an oxygen containing ambient. After drying at room temperature for 24 hours, the coated fiber was heated at $300{ }^{\circ} \mathrm{C}$ for 3 hours to cure the spin-on-glass. Figure. 4.5 shows experiment set up and a fiber sensor sample obtained using dip coating method.

\subsubsection{Thermal Evaporation}

Thermal evaporation is one of the most popular deposition methods. Thermal evaporation is one of the Physical Vapor Deposition (PVD) methods and typically uses a resistive heat source to evaporate a solid material in a vacuum environment to form a thin film on the substrate during a physical deposition process. The coating/target 
material is often located inside the evaporation source (boat, basket or coil). The target material is heated in a high vacuum environment to the evaporation point by joule heating of the resistive boat. The produced vapor pressure allows the molecules of the target material to move to the substrate and coat the surface of the substrate with a thin film. The reason to process thermal evaporation in high vacuum and low pressure environment inside the chamber is that collisions of vapor molecules with gas molecules in the chamber are undesirable during evaporation because they change the material vapor's direction of travel, adversely affecting the quality of thin film coverage. Also, a high vacuum improves the purity of the evaporated film. This can be explained by effect of the background gasses emerging in the contamination in the growing film. Several materials, such as Chromium, Silver, Nickel, etc. can be deposited on the surface of substrate using this method. This technique, due to its maturity, reproducibility, and large area is still attractive for glass forming chalcogenide glasses like as $\mathrm{S}(\mathrm{Se})$, Ge-S(Se) [106].

We used thermal evaporation to coat the tip of the fibers with ChGs in a Cressington $308 \mathrm{R}$ coating system at $10^{-6}$ mbar vacuum with an evaporation rate of 0.35 $\AA /$ s. The fiber was not heated during the film preparation. The thickness of the deposited film was estimated using the output from a quartz crystal microbalance. To check the composition of the deposited coating, ChG was also deposited on a single crystalline silicon substrate along with the fiber. Compared to the composition of the source material, the thin film had $\pm 1.5 \%$ compositional deviation as measured by an Energy Dispersive Spectroscopy (EDS) study. Once the deposition was completed, the fiber tip was dip-coated with spin-on-glass. After drying in room temperature for 24 hours, the coated fibers were heated at $300{ }^{\circ} \mathrm{C}$ for 3 hours to completely cure 


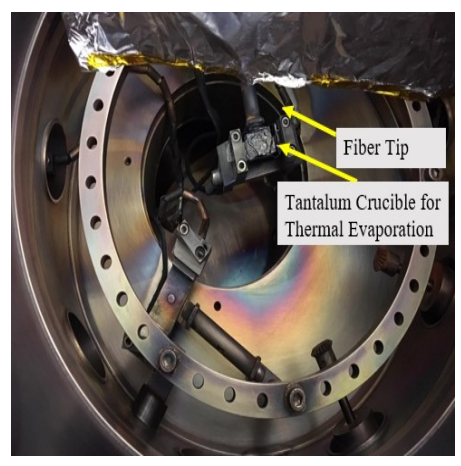

(a)

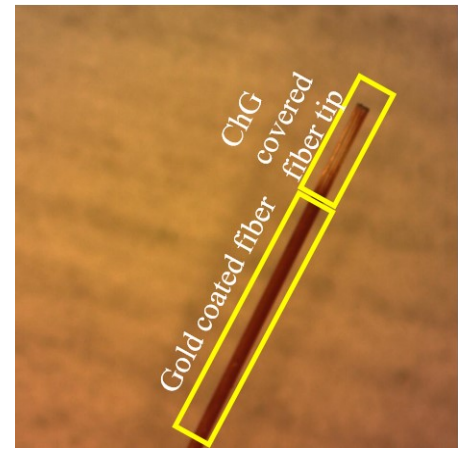

(b)

Figure 4.6: Thermal Evaporation fabrication method, a) Experiment setup to coat fiber tip with chalcogenide glass, b) chalcogenide glass covered fiber-tip.

the spin-on-glass. Figure. 4.6 shows experiment set up and a fiber sensor sample obtained using dip coating method. This vapor phase deposition process to form a ChG layer on a fiber tip is a standard process that leads to highly conformal and uniform in thickness coating. The thermally deposited sensors were used to benchmark the performance of the dip-coated devices.

\subsection{Experimental Setup}

The performance of the fabricated sensor devices was characterized using the experimental setup shown in Figure. 4.7.

Light from a $1550 \mathrm{~nm}$ wavelength selectable laser laser source (Santec, WSL-100) was injected into the fiber sensor through an optical circulator (THORLABS, Model: 6015-3-FC). The light power reflected from the fiber sensor was analyzed using an optical spectrum analyzer (Anritsu. MS9740A). The ChG-capped fiber tip itself was placed inside a high temperature-controlled tube furnace (Eurotherm 2116 controller). The furnace temperature was increased from room temperature $\left(\sim 25^{\circ} \mathrm{C}\right)$ up to 650 ${ }^{\circ} \mathrm{C}$ in $10{ }^{\circ} \mathrm{C} / \mathrm{min}$ steps. For evaluation of the real-time response of the sensor, the 


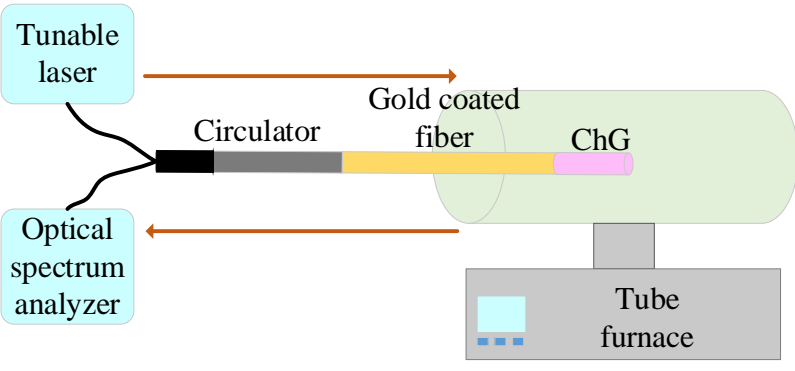

(a)

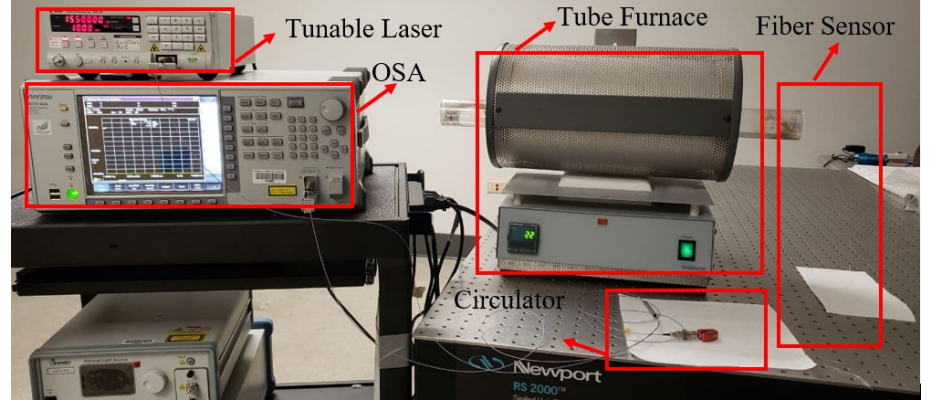

(b)

Figure 4.7: (a) Schematic of setup for testing the temperature performance of the fabricated sensors, (b) a picture of the actual device characterization setup.

temperature inside the furnace as a function of the time was tracked as well using a thermocouple.

\subsection{Results and Discussion}

The normalized measured reflected power (dotted black curve) as a function of time for two compositions- $\mathrm{Ge}_{30} \mathrm{~S}_{70}$ and $\mathrm{Ge}_{40} \mathrm{Se}_{60}$ are shown in Figure. 4.8 and Figure. 4.10, respectively. The normalized simulated reflected power with extracted refractive index profile from studying the particular ChGs as a function of time is also plotted (solid red curve). It can be seen from the figures that the measured and the simulated results agree very well. Since the proposed sensor works on the principle of the phase change of $\mathrm{ChG}$ material, which is highly temperature dependent, abrupt changes in 


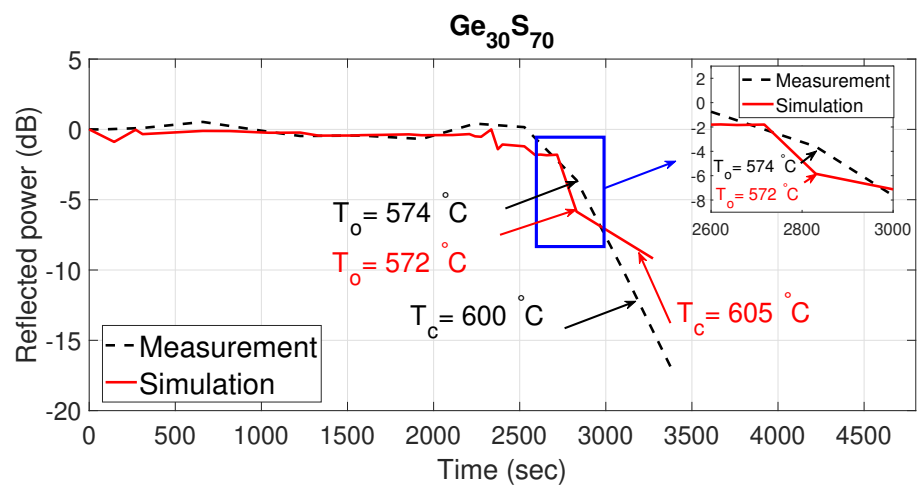

Figure 4.8: Simulated and measured normalized reflected power as a function of time with $\mathrm{Ge}_{30} \mathrm{~S}_{70}$ capped fiber tip.

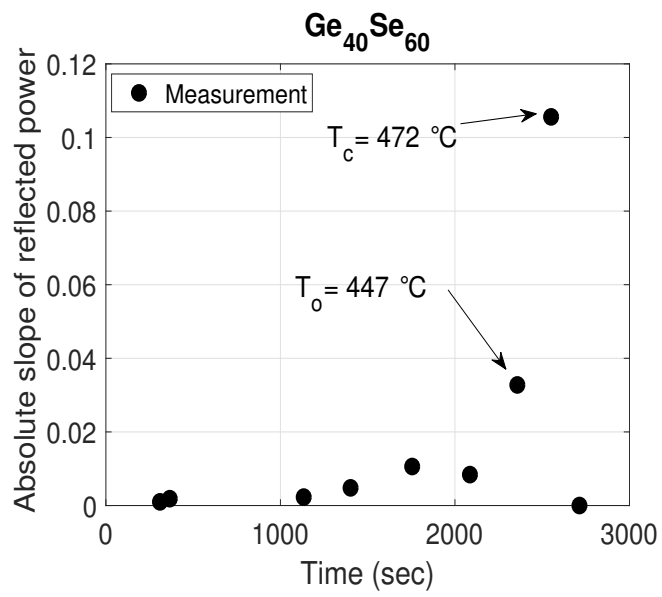

Figure 4.9: Temperature response of evaporated $\mathrm{Ge}_{40} \mathrm{Se}_{60}$ capped fiber-tip based temperature sensor.

the reflected power are observed, as expected. This Fresnel reflected power variation is due to temperature sensitivity of the ChG. These sudden changes and associated temperatures can be efficiently extracted from the sensor data by plotting the slope as a function of time. The slope method was used to measure the rate at which changes are taking place. It shows how reflected power changes in response to a change in temperature. It can be calcuted as the ratio of two values of reflected power versus 
corresponding temperatures as:

$$
\text { slope }=\frac{\text { changes in reflected power }}{\text { changes in temperature }}=\frac{P_{T_{2}}-P_{T_{1}}}{T_{2}-T_{1}}
$$

As we tracked the temperature as a function of time in the tube furnace, we can find the corresponding time for each temperature. Then, we can plot slope of reflected power as a function of time. Upward sloping shows that reflected power increases as temperature increases. Also downward sloping demonstrates that reflected power decreases as temperature increases. We used absolute slope to easily follow the changes in reflected power. A slope with a greater absolute value indicates big changes in reflected power as temperatures increase. For example, from the data regarding the $\mathrm{Ge}_{40} \mathrm{Se}_{60}$ composition shown in Figure. 4.10, a plot of the slope of measured reflected power as a function of time is shown in Figure. 4.9. This figure manifests a big growth in the absolute slope of the reflected power between the $T_{\mathrm{o}}$ and $T_{\mathrm{c}}$ due to the big structural reorganization occurring in a solid state from the onset of crystallization at $T_{\mathrm{o}}$ up to the full crystallization of the material at $T_{\mathrm{c}}$. Two peaks stand out at 2462 seconds and 2627 seconds, corresponding to the onset and peak crystallization temperatures of $447^{\circ} \mathrm{C}$ and $472{ }^{\circ} \mathrm{C}$ obtained from monitoring the temperature inside the furnace, which is close to the predicted temperatures of $446.6{ }^{\circ} \mathrm{C}$ and $472.3{ }^{\circ} \mathrm{C}$. The data related to the onset and peak crystallization temperatures calculated using the slope method for all studied compositions are shown in Table 4.1.

According to the measured refractive index data presented in Table 3.1, two crystallization temperatures for $\mathrm{Ge}_{40} \mathrm{~S}_{60}$ are expected. The experimental data, however, only provides one of the crystallization temperatures $\left(485^{\circ} \mathrm{C}\right)$, as shown in Table 4.1 . This is due to the fact that at the lower crystallization temperature, the structure of 


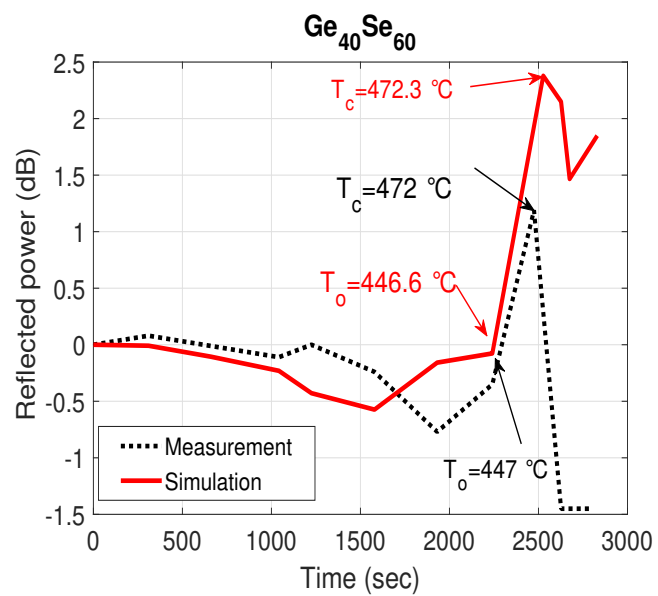

Figure 4.10: Simulated and measured normalized reflected power as a function of time with $\mathrm{Ge}_{40} \mathrm{Se}_{60}$ capped fiber tip.

the material is not strongly organized and thus no strong optical changes are observable. Table 4.1 also provides the expected temperatures error in the measured and the expected $T_{\mathrm{c}}$. It can be seen that except from the evaporated $\mathrm{Ge}_{33} \mathrm{Se}_{67}$ and dipcoated $\mathrm{Ge}_{30} \mathrm{Se}_{70}$ samples, all other samples are in good agreement within $\pm 0.4-2{ }^{\circ} \mathrm{C}$. The absolute slope of the reflected power decays because of crack formation during the crystallization process and increase in the interface roughness. These effects can be overcome by encapsulating the devices and/or melting the films and solidifying them in amorphous condition during the reversing process for re-usage of the devices. Based on our preliminary detailed studies of these materials and the collected Raman spectroscopy data, we suggest that this is a result of the fact that the stoichiometric composition has very homogeneous structure in the amorphous condition with low number of wrong bonds and this structure is maintained upon crystallization as well [93].

Similarly, the glasses with composition $\mathrm{Ge}_{30} \mathrm{Se}_{70}$, which have very close structure to the stoichiometric composition $\mathrm{Ge}_{33} \mathrm{Se}_{67}$, perform with small differences in 


\section{Table 4.1: Temperature response of Ge-S and Ge-Se tip coated optical fiber based temperature sensor.}

\begin{tabular}{|c|c|c|c|c|c|c|c|c|}
\hline Composition & Fabrication & $\begin{array}{c}\text { Expected } \\
\mathrm{T}_{\mathrm{o}}\end{array}$ & $\begin{array}{c}\text { Measured } \\
\mathrm{T}_{\mathrm{o}}\end{array}$ & $\begin{array}{c}\text { Time } \\
\mathrm{T}_{\mathrm{o}(\mathrm{sec})}\end{array}$ & $\begin{array}{c}\text { Expected } \\
\mathrm{T}_{\mathrm{c}}\end{array}$ & $\begin{array}{c}\text { Measured } \\
\mathrm{T}_{\mathrm{c}}\end{array}$ & $\begin{array}{c}\text { Time } \\
\mathrm{T}_{\mathrm{c}(\mathrm{sec})}\end{array}$ & $\begin{array}{c}\text { Error } \\
\mathrm{T}_{\mathrm{c}}\end{array}$ \\
\hline \multirow{2}{*}{$\mathrm{Ge}_{40} \mathrm{Se}_{60}$} & Dip-coated & 446.6 & 460 & 2589 & 472.3 & 472 & 2627 & 0.3 \\
\hline & Evaporated & 446.6 & 447 & 2462 & 472.3 & 472 & 2627 & 0.3 \\
\hline \multirow{2}{*}{$\mathrm{Ge}_{33} \mathrm{Se}_{67}$} & Dip-coated & 485.4 & 485 & 2646 & 527.7 & 528 & 2950 & 0.3 \\
\hline & Evaporated & 485.4 & 450 & 2527 & 527.7 & 485 & 2646 & 42.7 \\
\hline \multirow{2}{*}{$\mathrm{Ge}_{30} \mathrm{Se}_{70}$} & Dip-coated & 440.9 & 400 & 2241 & 470.4 & 450 & 2527 & 20.5 \\
\hline & Evaporated & 440.9 & 447 & 2462 & 470.4 & 460 & 2589 & 10.4 \\
\hline $\mathrm{Ge}_{40} \mathrm{~S}_{60}$ & Dip-coated & 480 & 450 & 2527 & 489 & 485 & 2646 & 4 \\
\hline $\mathrm{Ge}_{30} \mathrm{~S}_{70}$ & Dip-coated & 572 & 574 & 2830 & 605 & 600 & 3399 & 5 \\
\hline
\end{tabular}

the optical properties after crystallization due to the lack of strong reorganization of their structure. This is not the case for the $\mathrm{Ge}_{30} \mathrm{~S}_{70}$ compositions, because the non-stoichiometric members of the Ge-S system the 8-member $\mathrm{S}$ rings open up at higher temperatures to become a part of the tetrahedral backbone of the crystalline material, thus leading to observable change in the refractive index of this material. The crystallization kinetics and the formation of different structural units in these glasses are discussed in detail in $[93,107,108]$.

Moreover, it has been argued [96] that the optical and electrical property contrast due to phase change originates mostly from transformation in the structural medium-range order after crystallization. The crystalline $\operatorname{GeSe}(\mathrm{S})_{2}$ which appear after the phase change have predominantly corner-shared structure and are pseudo twodimensional when the low temperature forms of these materials crystallize [109,110]. In the high temperature dichalcogenides, the corner-sharing units are connected with edge-sharing building blocks [111]. Although, the kinetics and materials are studied extensively, the change in optical properties due to crystallization is not well understood in these compositions. For example, except for $\mathrm{x}=40$, Ge-S compositions show a decrease in refractive index after crystallization. We suggest that this is due to 
one more detail related to the $\mathrm{Ge}_{40} \mathrm{Se}(\mathrm{S})_{60}$ studied compositions - accordingly to the X-Ray Diffraction (XRD) studies besides GeSe $(\mathrm{S})_{2}$, the crystalline form occurring after the phase change contains also GeSe [93] which has orthorhombic structure [112]. In this structure, both atoms are threefold coordinated due to occurrence of dative bonds. It is for this reason that the Ge rich compositions react with bigger changes in optical properties after crystallization. In $\mathrm{Ge}_{33} \mathrm{Se}_{67}$, the change in optical properties due to crystallization is not distinct due to lack of medium range structural changes. For $\mathrm{Ge}_{30} \mathrm{Se}_{70}$, some previous studies [113] reported a reduction in refractive index after which is also the case for the examined thin films until their temperature reaches the crystallization temperature. These data are from ellipsometric studies of thin films on flat substrates, but the fiber devices showed an increase in reflected power after crystallization, which is direct evidence of the fact that the refractive index increases after crystallization of $\mathrm{Ge}_{30} \mathrm{Se}_{70}$. We assume that in the case of fiber there are additional interference effects occurring which influence the results measured from the fiber devices.

\subsection{Temperature Profile Estimation Using Array}

\section{Sensor}

Arranging the single $\mathrm{ChG}$ tip coated fibers (each fiber coated with a different composition) in an array structure as the schematic is shown in Figure. 4.11 and monitoring the reflected power from each fiber will help with the real-time detection of several temperatures inside extreme environments, thus mapping out the temperature profile.

Each of the synthesized ChG compositions has a specific crystallization temperature which allows for accurate monitoring and recording of real-time temperature 

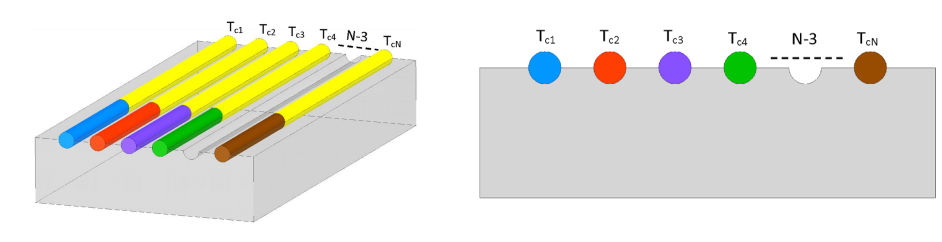

(a)

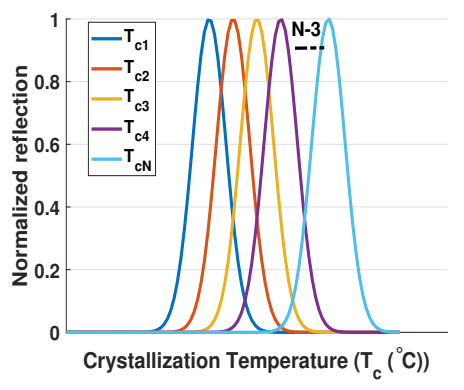

(b)

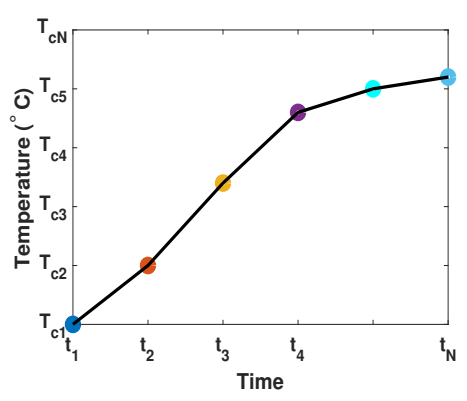

(c)

Figure 4.11: a) Optical fiber array with different ChG-capped fiber optics, b) Reflected output power in array structure, c) Temperature versus time.

profiles within a desired environment. As an example, the slope of the reflected power data from the array structure comprising of four optical fibers capped with four different ChGs $\left(\mathrm{Ge}_{40} \mathrm{Se}_{60}, \mathrm{Ge}_{30} \mathrm{~S}_{70}, \mathrm{Ge}_{40} \mathrm{~S}_{60}\right.$ and $\left.\mathrm{Ge}_{33} \mathrm{Se}_{67}\right)$ within a temperature range of $472{ }^{\circ} \mathrm{C}$ to $600{ }^{\circ} \mathrm{C}$ is shown in Figure. 4.12a. The proposed array sensor is arranged from ChG dip coated devices which show lower error in temperature response. Correlating the peak slope and times provides a temperature evolution chart as shown in Figure. 4.12b, while four distinct temperatures, $472{ }^{\circ} \mathrm{C}, 485{ }^{\circ} \mathrm{C}, 528{ }^{\circ} \mathrm{C}$ and 600 ${ }^{\circ} \mathrm{C}$ are recorded with this array structure. Increasing the array size to accommodate several other compositions of ChGs will enable real-time and precise monitoring of temperature with a higher temperature resolution. Since the fiber diameter is 125 $\mu m$, an array of $15-20$ sensors, leading to a temperature resolution less than $10{ }^{\circ} \mathrm{C}$ within $440-600{ }^{\circ} \mathrm{C}$ will result in the sensor node area size smaller than $2.125 \mathrm{~mm}^{2}$, 


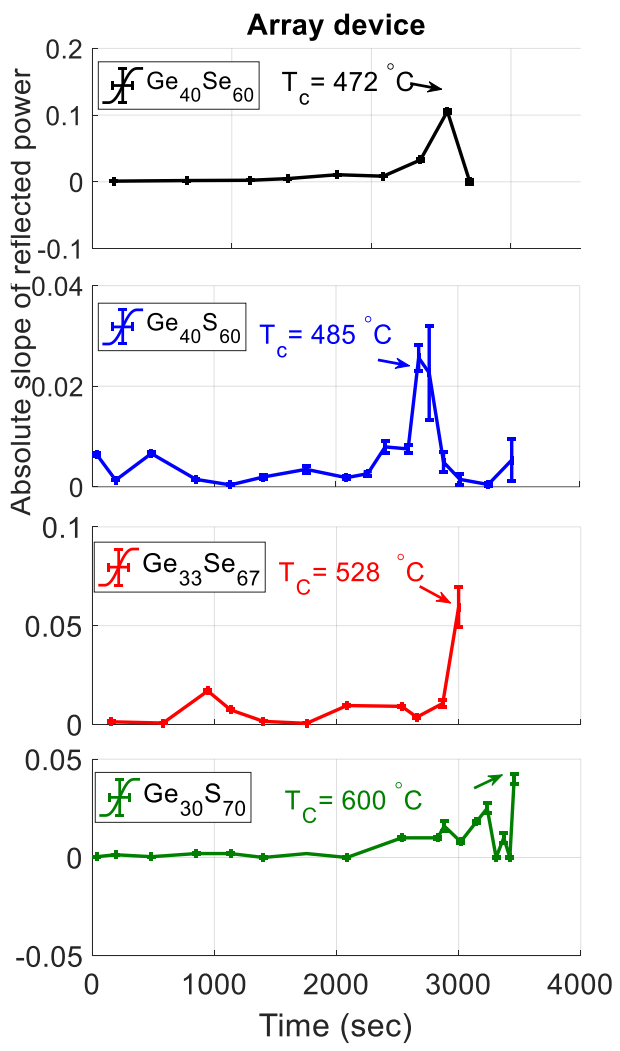

(a)

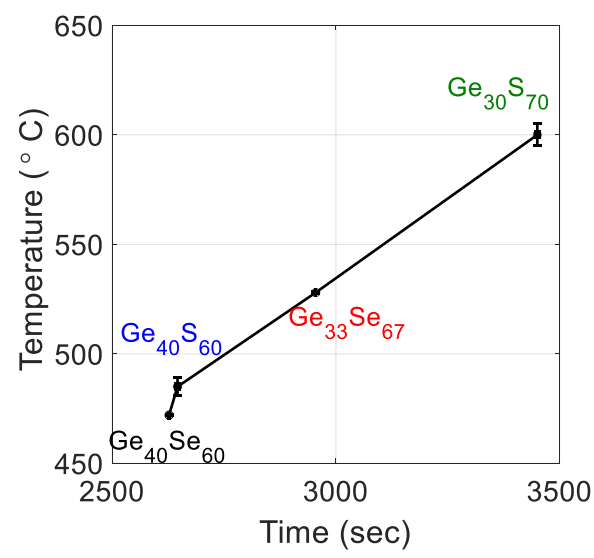

(b)

Figure 4.12: a) Temperature response of the sensor array, b) Monitored temperature trend as a function of time using array structure. 
which makes this array easily deployable within structures. To realize these array sensors, the individual fibers can be packed using a fiber groove array structure. A 1:N power splitter coupled with $\mathrm{N}$ circulators can be used to couple the light individually into each of the $N$ fibers and collect the $\mathrm{N}$ reflected powers for analysis. Table 4.2 shows the comparison between performance of proposed optical fiber based temperature sensor with other studies.

\subsection{Summary}

In this chapter, we described the fabrication process of the fiber based temperature sensor. Sensor devices with 6 different in-house synthesized ChGs are fabricated. The temperature response from each of the devices showed the same results as we expected from simulations. Sensors arranged in an array structure was used to follow the trend of the temperature changes in a tube furnace. A slope-based data analysis method was developed to analyze the reflected power level difference in amorphous and crystallization phases of each of the Ge-S Ge-Se capped devices. The measured results confirm the functionality of the proposed structure. In the next chapter, we propose the second temperature sensor architecture based on planar optical waveguides. 


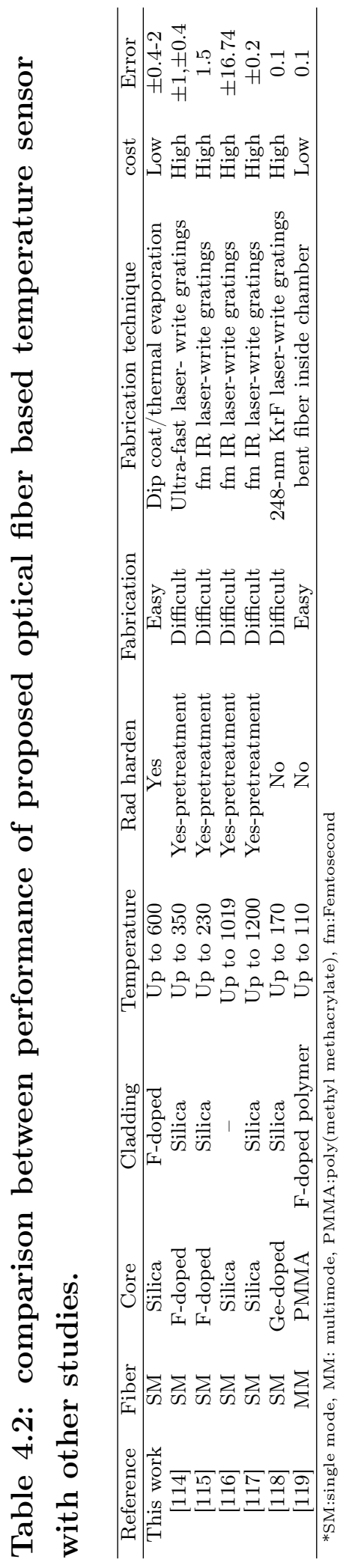




\section{CHAPTER 5:}

\section{MODELING AN INTEGRATED SILICON WAVEGUIDE:CHG BASED COMPACT PLASMONIC TEMPERATURE SENSOR}

In this chapter, modeling details on the silicon waveguide:ChG based temperature sensor are provided. The waveguide is covered with six different in-house synthesized ChG inks. The effect of the each of the compositions on the transmitted power of the waveguide is studied. Different complex refractive index values in the amorphous and crystalline phases of the ChGs affect the transmitted power. The simulation results confirm that in the crystalline phase of Ge-S and Ge-Se, the metallic behavior of these compositions leads to creation of plasmonic modes which are highly lossy. The extinction ratio indicates that within a small length of the waveguide, abrupt changes in transmitted power occur around the crystalline temperature of ChGs. Changes in output power can be used to determine the ambient temperature.

\subsection{Optical Waveguide Structure}

Here, the designs of the plasmonic waveguide based temperature sensors on siliconon-insulator (SOI) substrate are presented. The proposed sensor's operation is based on the phase change of $\mathrm{ChG}$ from amorphous to crystalline condition due to an in- 
crease in the ambient temperature beyond ChG's crystallization temperature. The proposed design and simulation of several structures of plasmonic based temperature sensor are addressed in this section. A SOI wafer is used for all devices. A commercial SOI with a buried oxide thickness of $3 \mu \mathrm{m}$ and silicon device layer thickness of 0.22 $\mu m$ is selected for simulation and fabrication. The thick buried oxide (BOX) ensures negligible light leakage toward the Si handle substrate at a wavelength of $1.55 \mu \mathrm{m}$. The thin upper silicon device layer is used to define an optical waveguide structure. Silicon has a much higher refractive index than our ChGs, leading to light confinement within the silicon waveguide. We designed our waveguides for a single-mode operation at $1.55 \mu \mathrm{m}$ to prevent losses from higher order modes. Specific design parameters of each device are determined using the simulation and iterative optimization performed using PhotonDesign's FIMMWAVE and FIMMprop simulation software packages which uses EigenMode Expansion (EME) method capable of giving solutions to wave equations which are fully vectorial and fully bi-directional.The software allows us to define a cross-section and top view of the design and then use mode solver to find the fundamental modes. We can obtain mode parameters, such as mode intensity, effective index, loss and propagation through this modeling software. The operating principle of the devices are described below.

Assuming ideal behavior from ChG glasses, below the crystallization temperature, in their amorphous state, $\mathrm{ChG}$ glasses demonstrate good dielectric behavior. In this configuration, the fundamental mode is confined in the silicon waveguide, and the mode propagates along the waveguide with nominal loss of $\sim 2.5-3.0 \mathrm{~dB} / \mathrm{cm}$. When the temperature of the ambient is above the crystallization transition temperature of $\mathrm{ChG}$, the material crystallizes and exhibits excellent conductive characteristics. 
The metallic behavior of ChG glasses in their crystalline phase generate surface plasmon polarition (SPP) modes in the plasmonic waveguide. These SPPs are highly localized at the metal:dielectric interface due to their sensitivity to dielectric properties near the metal surface. The plasmonic modes provide confinement beyond the diffraction limit, and are characterized by very large and abrupt propagation losses ( $1-10 \mathrm{~dB} / \mu \mathrm{m})$ [113]. The large change in the propagation loss from $\sim 2.5 \mathrm{~dB} / \mathrm{cm}$ to $>1 \mathrm{~dB} / \mu m$, leads to the determination of the crystallization temperature $T_{c}$ accurately. This remarkable property, together with CMOS-compatible fabrication of the silicon device, promises a very reliable, low-cost, and reusable sensor network. Since the complex refractive index of ChG glasses, and the change in their values transitioning from amorphous to crystalline states vary significantly based on the glass composition, we investigated different device designs that will provide best performance for each of those categories of refractive indices. Since the complex refractive index of GST glass $\left(\mathrm{Ge}_{2} \mathrm{Sb}_{2} \mathrm{Te}_{5}\right)$ is widely characterized, to begin, we used this glass in our designs. The refractive indices of $\mathrm{SiO}_{2}$ and $\mathrm{Si}$ at $1550 \mathrm{~nm}$ wavelength are $n_{\mathrm{SiO}_{2}}=1.45$ and $n_{S i}=3.47$ respectively. The refractive index and extinction coefficient of ChG (which is $\mathrm{Ge}_{2} \mathrm{Sb}_{2} \mathrm{Te}_{5}$ ) in amorphous and crystalline phases are $n_{\text {amor }}=2.14+\mathrm{i} 0.17$ and $n_{\text {crys }}=2.62+\mathrm{i} 2.11[120]$. The imaginary part of the refractive index estimates the loss of the waveguide. Note that this ChG characteristic deviates from an ideal case wherein the imaginary part for the amorphous state is assumed to be 0 or negligible. Thus, lossy behavior is expected for the waveguides in both states. Still, as will be shown in the simulation results in this section, an extinction ratio $>10 \mathrm{~dB}$ is achieved within an ultra-short length of the waveguide $(\sim<5 \mu \mathrm{m})$, compared to the several $m m$ long lengths that would be required with conventional waveguides. This makes 
the sensor array extremely compact and easier to embed or deploy. The waveguide device is designed to operate in single mode region. Single mode structure provides many advantages in the behavior of the guided light as well as being compatible with existing telecommunication systems. The fundamental mode of a waveguide, carries the majority of optical power and is the most well confined field distribution. The high confinement of the fundamental mode corresponds to a small portion of the field existing outside of the core (evanescent tails), minimizing losses from sidewall interference scattering. In the following subsections, the work performed in the designing of a waveguide based sensor is presented.

\subsubsection{Design 1 (ChG covering silicon waveguide)}

This design is a general design that is applicable to either a small or a large change in complex refractive index values, as long as the real part of the ChG refractive index always remains below silicon's refractive index, at least in one of the two glass phases. In this structure, $\mathrm{Si}$ waveguide is surrounded by $\mathrm{ChG}$. The $\mathrm{ChG}$ is covered by a passivation layer, which is $\mathrm{SiO}_{2}$ in our case. A schematic cross section https://www.overleaf.com/projectand dimension of single mode design is illustrated in Figure. 5.1 with optimized design parameters shown in Table 5.1.

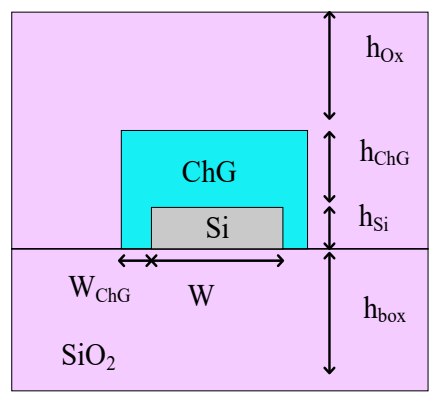

(a)

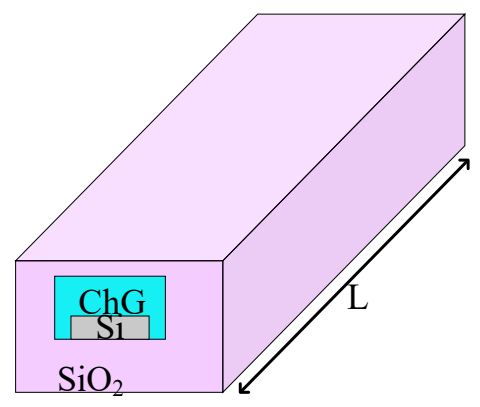

(b)

Figure 5.1: a) Cross section of Design 1, b) 3D view of Design 1. 
Table 5.1: Optimum design parameters obtained for Design 1.

\begin{tabular}{cc}
\hline Parameters & Value $(\mu m)$ \\
\hline $\mathrm{W}$ & 0.45 \\
\hline $\mathrm{W}_{C h G}$ & 0.1 \\
\hline $\mathrm{h}_{s i}$ & 0.22 \\
\hline $\mathrm{h}_{C h G}$ & 0.3 \\
\hline $\mathrm{h}_{O x}$ & 0.5 \\
\hline $\mathrm{h}_{b o x}$ & 2 \\
\hline $\mathrm{L}$ & 5 \\
\hline
\end{tabular}

We studied the behavior of the two fundamental modes - transverse electric (TE) and transverse magnetic (TM) modes propagating along the single mode silicon waveguide in amorphous phase of chalcogenide. Figure. 5.2 and Figure. 5.3 show the intensity distribution and output power in amorphous phase for TE and TM modes in amorphous phase of GST, respectively. Figure. 5.2a shows the cross sectional intensity distribution (mode profile) of the TE mode in the waveguide. The result shows that the TE mode is confined within the silicon waveguide. Figure. 5.2b and Figure. 5.2c, respectively, show the propagation and the associated propagation loss along the waveguide. Parametric study confirms that by increasing the length of the waveguide, the output power decreases which reduces the output power. The TM mode in the amorphous phase is confined near the interface of $\mathrm{Si}$ and $\mathrm{ChG}$, but majorly within the silicon waveguide, as shown in Figure. 5.3a. Figure. 5.3b and Figure. 5.3c, respectively, show the propagation and the associated propagation loss along the waveguide. Table 5.3 shows designed waveguide loss in amorphous phases of ChG in TM mode. 


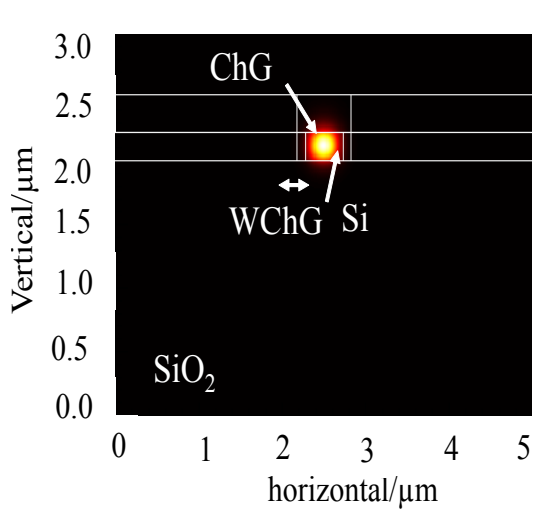

(a)

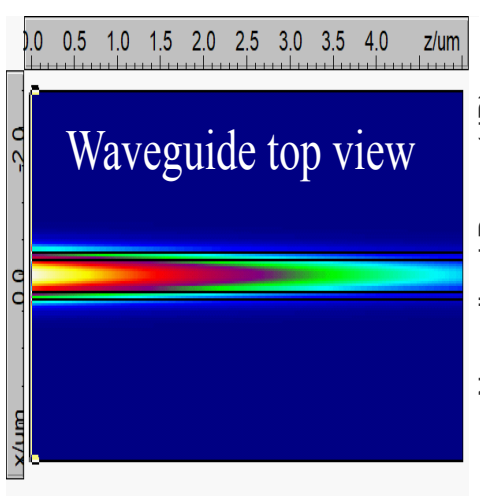

(b)

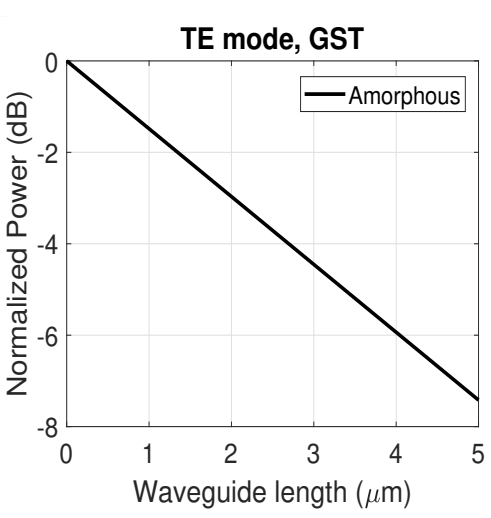

(c)

Figure 5.2: Amorphous Phase, a) Intensity profile of TE mode, b) Intensity distribution along the waveguide, and c) Output power of TE mode as a function of the length of the waveguide.

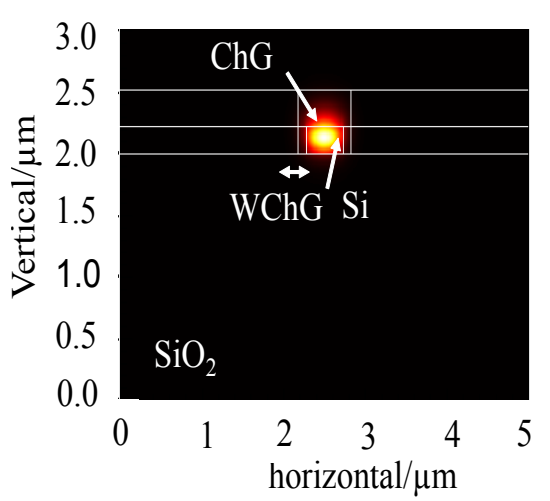

(a)

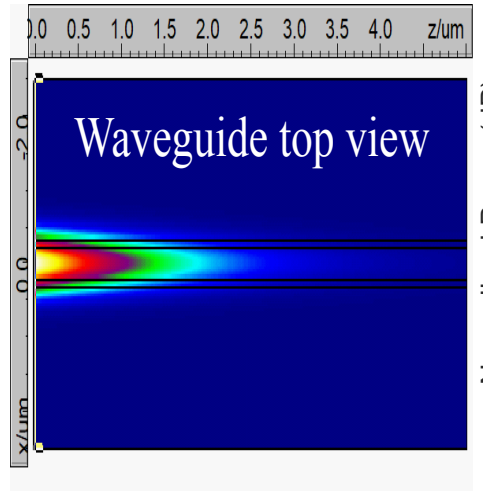

(b)

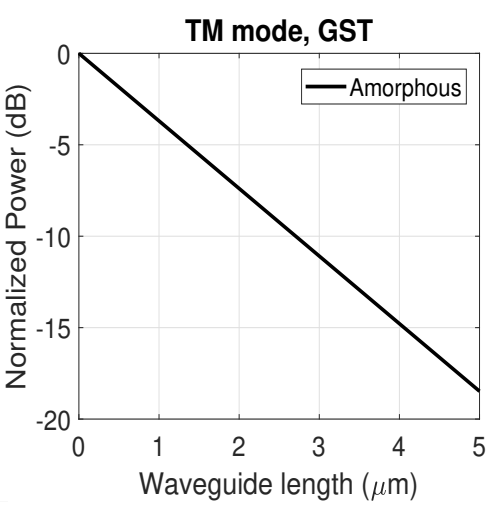

(c)

Figure 5.3: Amorphous phase, a) Intensity profile of TM mode, b) Intensity distribution along the waveguide, and c) Output power of TM mode as a function of the length of the waveguide.

Table 5.2: Amorphous phase waveguide loss of Design 1.

\begin{tabular}{cccc}
\hline & $\begin{array}{c}\text { Length } \\
(\mu \mathrm{m})\end{array}$ & $\begin{array}{c}\text { Transmission loss } \\
(\mathrm{dB})\end{array}$ & $\begin{array}{c}\text { Propagation loss } \\
(\mathrm{dB} / \mu \mathrm{m})\end{array}$ \\
\hline Fundamental TE mode & 3 & -5.46 & -1.82 \\
\hline Fundamental TM mode & 3 & -11.34 & -3.78 \\
\hline
\end{tabular}


When the temperature of the ChG increases beyond the crystallization transition temperature, the material crystallizes and exhibits metallic characteristics. In this configuration, the fundamental TE and TM modes disappear, and plasmonic modes appear at the interface between silicon and the metallic $\mathrm{ChG}$ material, as shown in Figure. 5.4a and Figure. 5.5a, respectively. Thus, the output power for TE and TM modes drop rapidly at the output, as shown in Figure. 5.4 (b-c) and Figure. 5.5(bc), respectively. By increasing the length of the waveguide beyond $3 \mu m$, no light power is observed at the output. This remarkable property, together with CMOScompatible fabrication of the silicon device, promises a very reliable, low-cost, and reusable compact sensor network. In Table 5.3 waveguide losses in two fundamental modes in crystalline phase of $\mathrm{ChG}$ is presented. Table 5.4 shows the summary of waveguide loss at a length of $3 \mu \mathrm{m}$ in two different phases of $\mathrm{ChG}$ in the proposed structure. This table reflects more than an order of magnitude increase in the loss for the crystalline phase compared to the amorphous phase. This high loss in crystalline phase compared to the amorphous phase is due to the difference in the extinction coefficients. Extinction coefficient in crystalline phase is greater than in the amorphous phase, which means that higher loss is expected in the crystalline phase. Figure. 5.6 shows the calculated extinction ratio (TM mode results shown as an example), which is the ratio of the output power in the amorphous phase to the output power in the crystalline phase, for the TM mode. Our devices demonstrates a high extinction ratio in a $3 \mu \mathrm{m}$ long waveguide, thus demonstrating the compactness of our designs. 


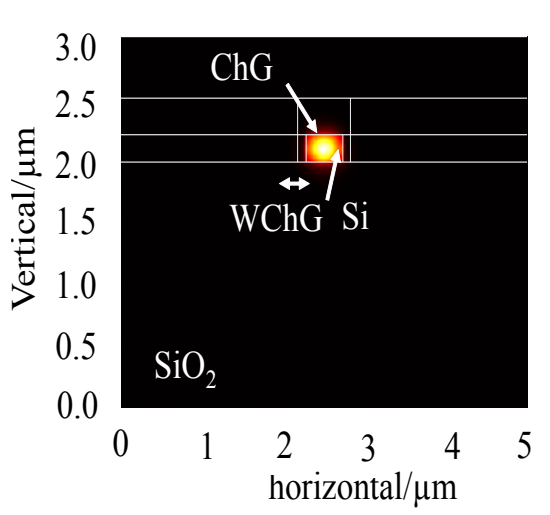

(a)

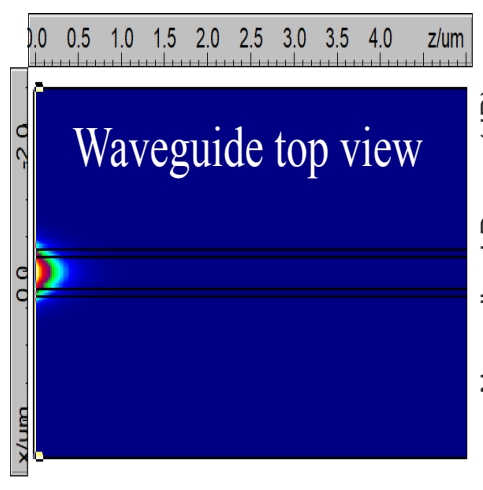

(b)

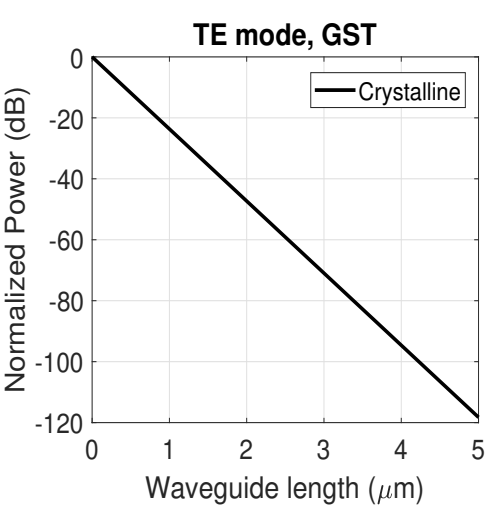

(c)

Figure 5.4: Crystalline Phase, a) Intensity profile of TE mode, b) Intensity distribution along the waveguide, and c) Output power of TE mode as a function of the length of the waveguide.

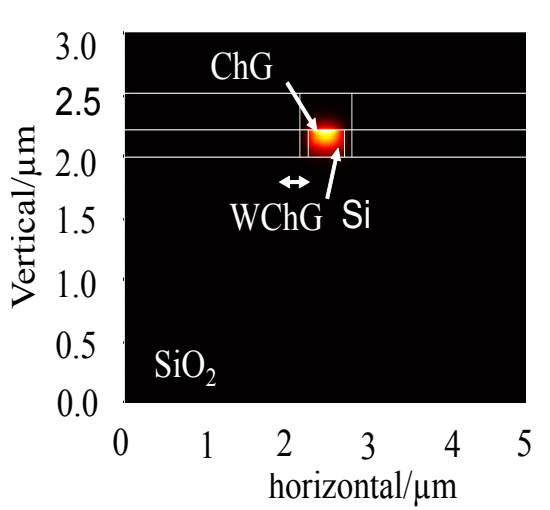

(a)

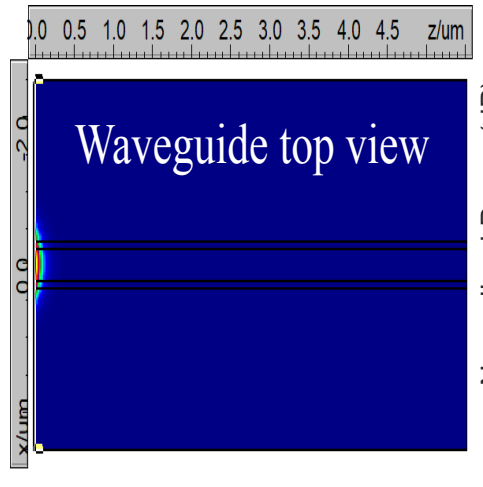

(b)

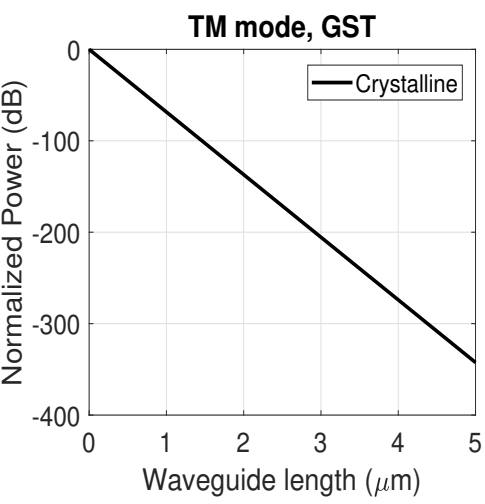

(c)

Figure 5.5: Crystalline Phase, a) Intensity profile of TM mode, b) Intensity distribution along the waveguide, and c) Output power of TM mode as a function of the length of the waveguide.

Table 5.3: Crystalline phase waveguide loss of Design 1.

\begin{tabular}{cccc}
\hline & $\begin{array}{c}\text { Length } \\
(\mu \mathrm{m})\end{array}$ & $\begin{array}{c}\text { Transmission loss } \\
(\mathrm{dB})\end{array}$ & $\begin{array}{c}\text { Propagation loss } \\
(\mathrm{dB} / \mu \mathrm{m})\end{array}$ \\
\hline Fundamental TE mode & 3 & -95.05 & -31.68 \\
\hline Fundamental TM mode & 3 & -207.73 & -69.24 \\
\hline
\end{tabular}


Table 5.4: Summary of losses for Design 1.

\begin{tabular}{ccc}
\hline & TE & TM \\
\hline Amorphous Phase Propagation Loss & $-1.82 \mathrm{~dB} / \mu m$ & $-3.78 \mathrm{~dB} / \mu m$ \\
\hline Crystalline Phase Propagation Loss & $-31.68 \mathrm{~dB} / \mu m$ & $-69.24 \mathrm{~dB} / \mu m$ \\
\hline
\end{tabular}

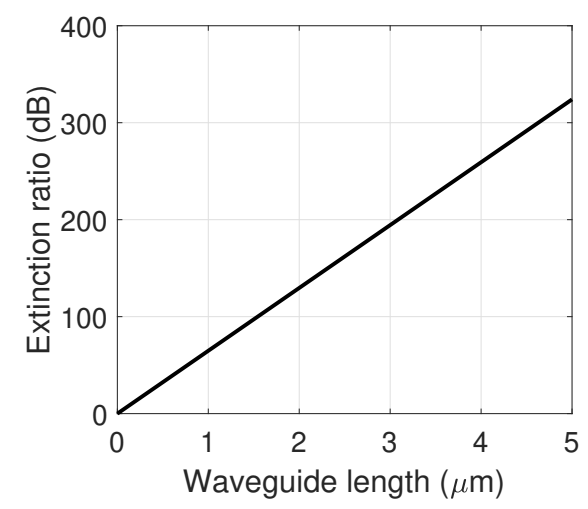

Figure 5.6: Extinction ratio of Design 1 in TM mode.

\subsubsection{Design 2 (A thin ChG layer sandwiched between Si waveg- uide and metal)}

In this structure, a thin ChG layer is sandwiched between $\mathrm{Si}$ and another metal, say gold (any metal choice will work for this design). Gold is chosen as a representative metal in the simulations since its material properties are readily available in the software library. The refractive index of the gold is $n_{A u}=0.58+\mathrm{i} 9.86$ at $1550 \mathrm{~nm}$ wavelength. As long as the real part of the refractive index is much smaller than silicon refractive index, this design has shown promise in our simulations. A schematic cross section of this design is illustrated in Figure. 5.7. The optimized device dimensions are shown in Table 5.5. 
Table 5.5: Optimum design parameters obtained for Design 2.

\begin{tabular}{cc}
\hline Parameters & Value $(\mu m)$ \\
\hline $\mathrm{W}$ & 0.45 \\
\hline$h_{A u}$ & 0.1 \\
\hline $\mathrm{h}_{s i}$ & 0.22 \\
\hline $\mathrm{h}_{C h G}$ & 0.3 \\
\hline $\mathrm{h}_{O x}$ & 0.5 \\
\hline $\mathrm{h}_{\text {box }}$ & 2 \\
\hline $\mathrm{L}$ & 5 \\
\hline
\end{tabular}

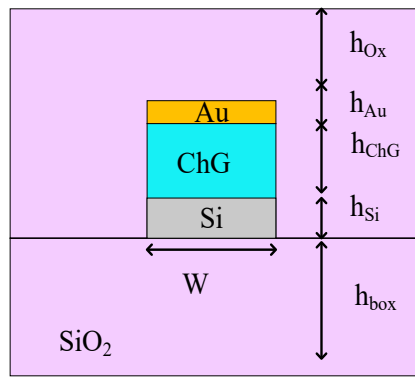

(a)

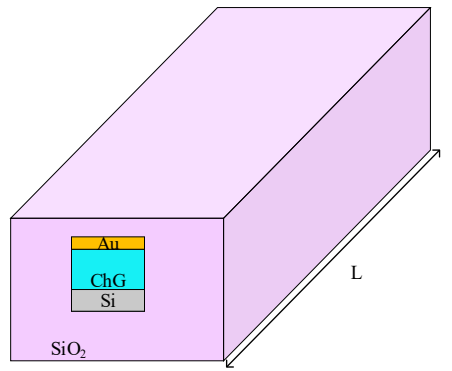

(b)

Figure 5.7: a) Cross section of Design 2, b) 3D view of Design 2.

Figure. 5.8a shows the cross sectional intensity distribution in the waveguide. The simulation result shows that the TE mode is confined within the silicon waveguide. This is due to high refractive index of $\mathrm{Si}$ compared to $\mathrm{ChG}$ and $\mathrm{Au}$. Figure. 5.8b and Figure. $5.8 \mathrm{c}$ show the propagation and propagation loss along the waveguide. In the amorphous phase, TM mode is confined in the thin dielectric $(\mathrm{ChG})$, as shown in Figure. 5.9a and relatively higher loss propagation occurs compared to the TE mode, as illustrated in Figure. 5.9b due to the interaction of the TM mode with the gold metal on top. Increasing the length of the waveguide decreases the output power exponentially, as shown Figure. 5.9c. The propagation loss in amorphous phase of ChG is shown in Table 5.6. 


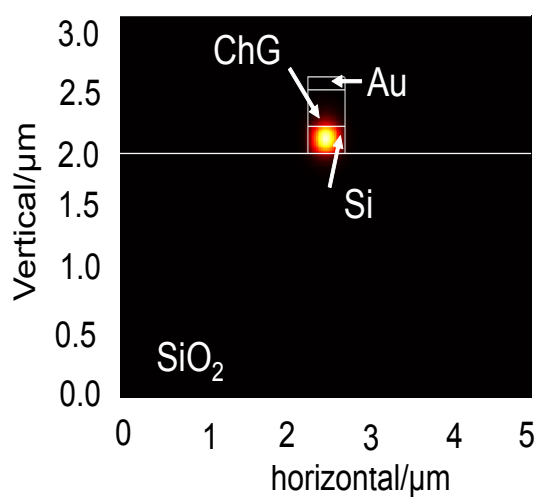

(a)

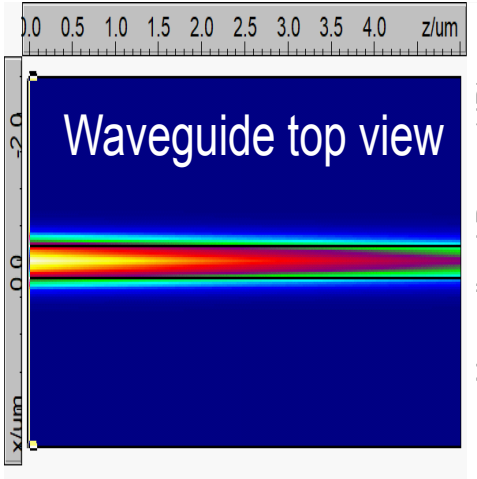

(b)

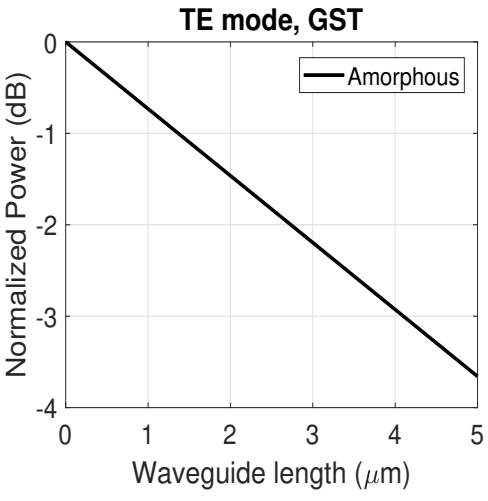

(c)

Figure 5.8: Amorphous phase a) Intensity profile of TE mode, b) Intensity distribution along the waveguide, and c) Output power of TE mode as a function of the length of the waveguide.

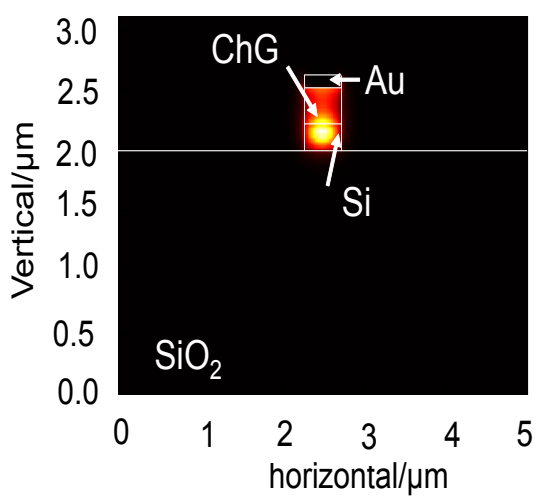

(a)

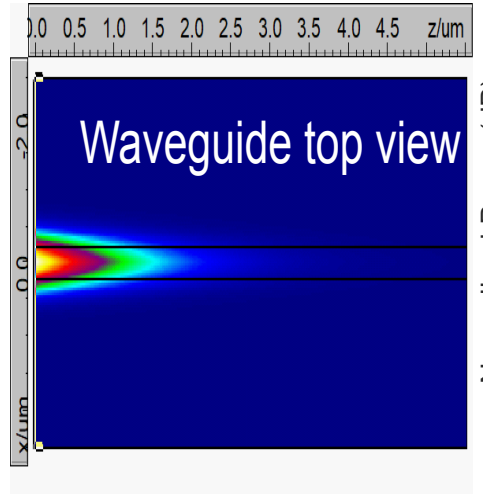

(b)

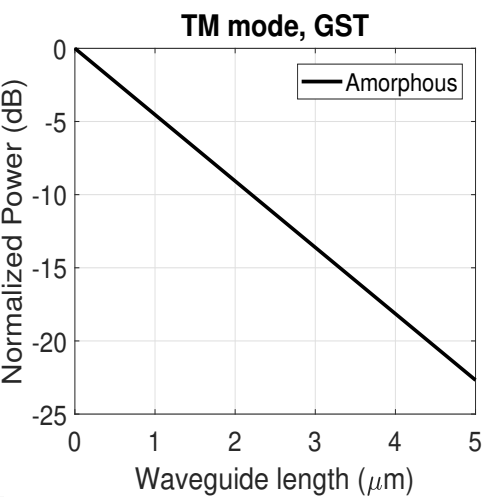

(c)

Figure 5.9: Amorphous phase a) Intensity profile of TM mode, b) Intensity distribution along the waveguide, and c) Output power of TM mode as a function of the length of the waveguide.

Table 5.6: Amorphous phase waveguide loss of Design 2.

\begin{tabular}{cccc}
\hline & $\begin{array}{c}\text { Length } \\
(\mu \mathrm{m})\end{array}$ & $\begin{array}{c}\text { Transmission loss } \\
(\mathrm{dB})\end{array}$ & $\begin{array}{c}\text { Propagation loss } \\
(\mathrm{dB} / \mu m)\end{array}$ \\
\hline Fundamental TE mode & 3 & -2.15 & -0.71 \\
\hline Fundamental TM mode & 3 & -9.75 & -3.25 \\
\hline
\end{tabular}


At the crystallization transition temperature, $\mathrm{ChG}$ changes its phase and exhibits metallic characteristics. So, due to the interaction of TE mode with a highly metallic material, the loss increases from $0.71 \mathrm{~dB} / \mu \mathrm{m}$ in the amorphous case to $8.45 \mathrm{~dB} / \mu \mathrm{m}$ in crystalline phase of ChG (Figure. 5.10). For the TM mode, the plasmonic mode shifts to the interface between silicon and the metallic ChG. For plasmonic modes, the loss is directly proportional to the refractive index of the dielectric material the plasmonic modes interact with. Therefore, compared to the amorphous case, wherein the plasmonic mode interacted with $\mathrm{ChG}$, the loss for the plasmonic mode for the crystalline phase will be higher. Thus, the output power for TM mode drops rapidly at the output from $3.25 \mathrm{~dB} / \mu m$ to $66.94 \mathrm{~dB} / \mu m$, as shown in Figure. 5.11. Table 5.7 shows losses of waveguide when $\mathrm{ChG}$ is as metal. The summary of propagation loss of design 2 is presented in Table 5.8. This device demonstrate a high extinction ratio greater than $10 \mathrm{~dB}$ within a compact $3 \mu \mathrm{m}$ long waveguide.

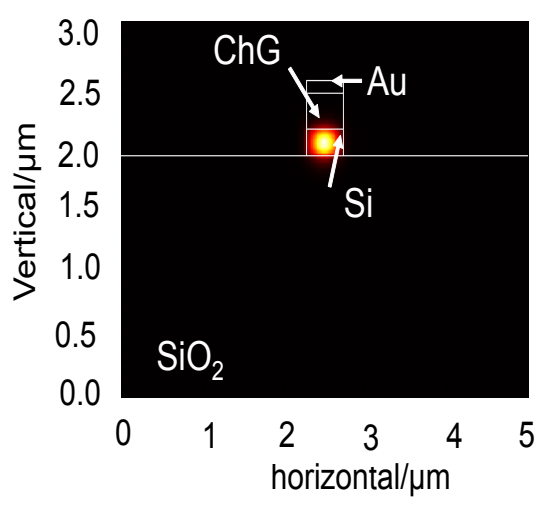

(a)

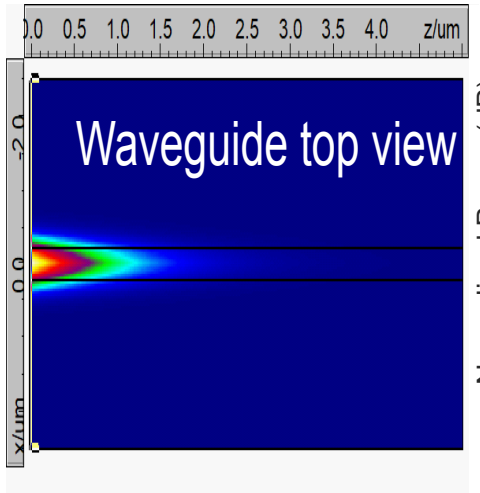

(b)

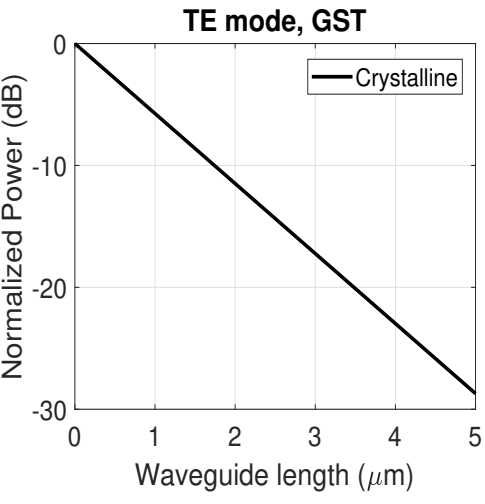

(c)

Figure 5.10: Crystalline phase a) Intensity profile of TE mode, b) Intensity distribution along the waveguide, and c) Output power of TE mode as a function of the length of the waveguide. 
Table 5.7: Crystalline phase waveguide loss of Design 2.

\begin{tabular}{cccc}
\hline & $\begin{array}{c}\text { Length } \\
(\mu \mathrm{m})\end{array}$ & $\begin{array}{c}\text { Transmission loss } \\
(\mathrm{dB})\end{array}$ & $\begin{array}{c}\text { Propagation loss } \\
(\mathrm{dB} / \mu m)\end{array}$ \\
\hline Fundamental TE mode & 3 & -25.36 & -8.45 \\
\hline Fundamental TM mode & 3 & -200.84 & -66.94 \\
\hline
\end{tabular}

Table 5.8: Summary of losses for Design 2.

\begin{tabular}{lcc}
\hline & TE & TM \\
\hline Amorphous Phase Propagation Loss & $-0.71 \mathrm{~dB} / \mu m$ & $-3.25 \mathrm{~dB} / \mu \mathrm{m} \mu m$ \\
Crystalline Phase Propagation Loss & $-8.45 \mathrm{~dB} / \mu m$ & $-66.94 \mathrm{~dB} / \mu m$ \\
\hline
\end{tabular}

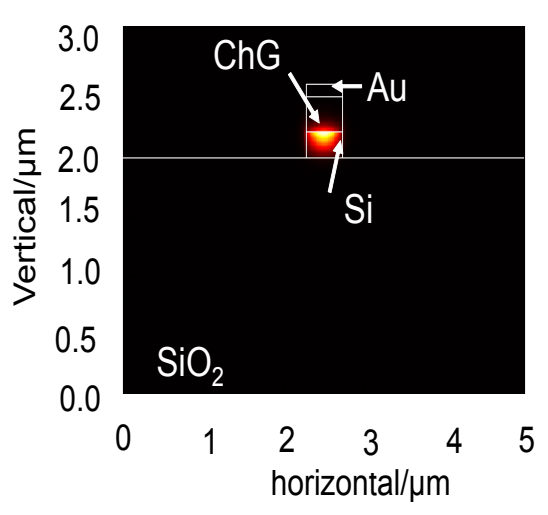

(a)

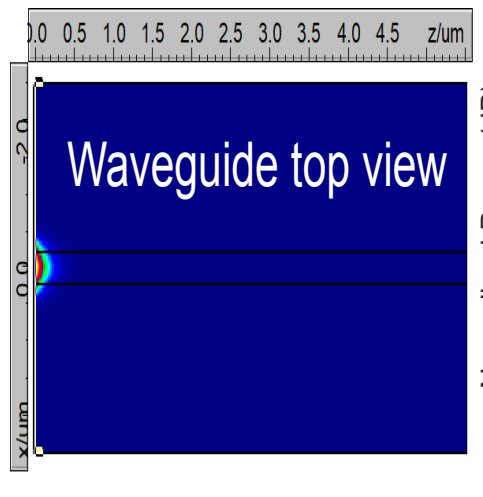

(b)

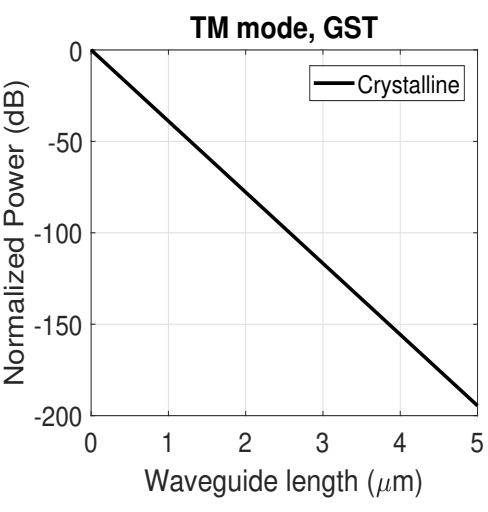

(c)

Figure 5.11: Crystalline phase a) Intensity profile of TM mode, b) Intensity distribution along the waveguide, and c) Output power of TM mode as a function of the length of the waveguide.

\subsubsection{Design 3 (ChG glass patterned on the top surface of silicon waveguide)}

This design is similar to Design 1, except that it is expected to work for polarized light and where dense integration of waveguides is required. A schematic cross section of this design is shown in Figure. 5.12. 
Table 5.9: Optimum design parameters obtained for Design 3.

\begin{tabular}{cc}
\hline Parameters & Value $(\mu m)$ \\
\hline $\mathrm{W}$ & 0.45 \\
\hline $\mathrm{h}_{s i}$ & 0.22 \\
\hline $\mathrm{h}_{C h G}$ & 0.3 \\
\hline $\mathrm{h}_{O x}$ & 0.5 \\
\hline $\mathrm{h}_{\text {box }}$ & 2 \\
\hline $\mathrm{L}$ & 5 \\
\hline
\end{tabular}

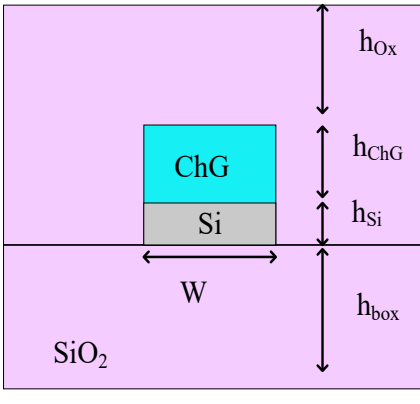

(a)

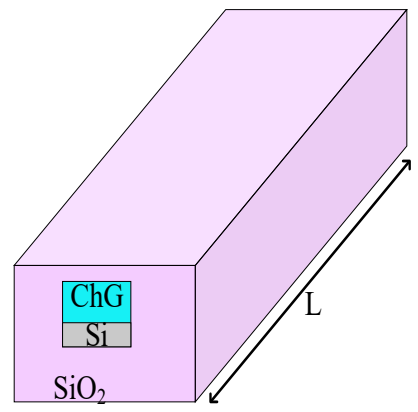

(b)

Figure 5.12: a) Cross section of Design 3, b) 3D view of Design 3.

The optimized design parameters are shown in Table 5.9. The TM mode interacts with ChG much more than the TE mode since the sidewalls of the silicon waveguide are not covered by the ChG material. Figure. 5.13 shows the intensity distribution, propagation and output power in amorphous phase for TM mode. The result shows that the TE mode is confined within the silicon waveguide. Figure. 5.13b and Figure. $5.13 \mathrm{c}$ show the propagation loss along the waveguide, as a function of the length of the waveguide. Parametric study confirms that by increasing the length of the waveguide, the output power decreases which reduces the output power. The fundamental mode propagates along the waveguide with minimum loss. The value of losses in design 3 for an amorphous phase of ChG is shown in Table 5.10. 
Table 5.10: Amorphous phase waveguide loss of Design 3.

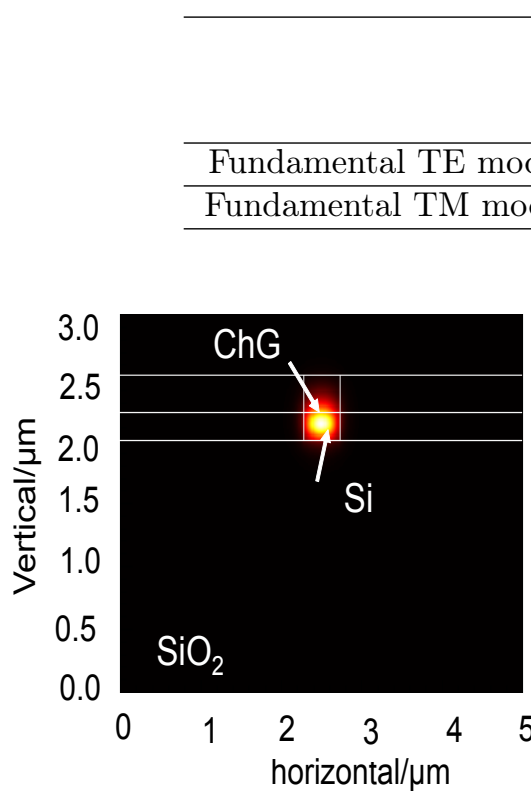

(a)

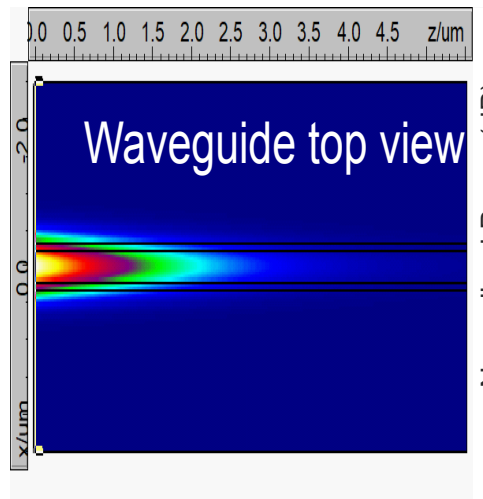

(b)

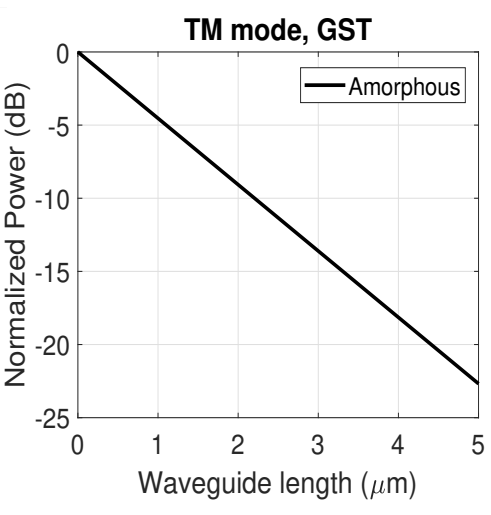

(c)

Figure 5.13: Amorphous phase a) Intensity profile of TM mode, b) Intensity distribution along the waveguide, and c) Output power of TM mode as a function of the length of the waveguide.

In the crystalline phase, ChG acts like metal, so we expect the SPPs at the interface of $\mathrm{Si}$ and $\mathrm{ChG}$. TM mode is tightly confined at the interface between silicon and the metallic ChG, as shown in Figure. 5.14a. Thus, higher loss appears and the output power drops dramatically [Figure. 5.14b]. Table 5.11 shows the propagation losses in the crystalline phase of ChG. The summary of losses for design 3 in TM mode is shown in Table 5.12. A comparision between the propagation losses in different structures of plasmonic waveguides in two fundamentals modes (TE and TM) is provided in Table 5.12. 
Table 5.11: Waveguide loss of Design 3.

\begin{tabular}{lccc}
\hline & $\begin{array}{c}\text { Length } \\
(\mu \mathrm{m})\end{array}$ & $\begin{array}{c}\text { Transmission loss } \\
(\mathrm{dB})\end{array}$ & $\begin{array}{c}\text { Propagation loss } \\
(\mathrm{dB} / \mu \mathrm{m})\end{array}$ \\
\hline Fundamental TM mode (amorphous) & 3 & -9.75 & -3.25 \\
\hline Fundamental TM mode (Crystalline) & 3 & -155.91 & -51.97 \\
\hline
\end{tabular}

Table 5.12: Summary of losses for Design 3.

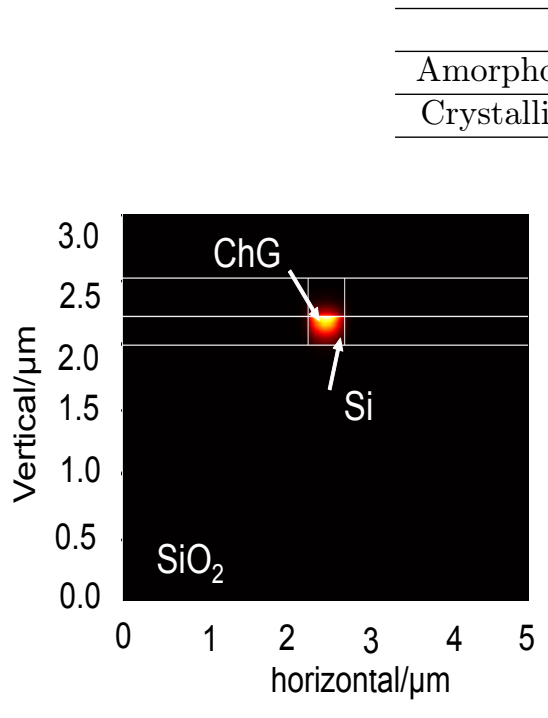

(a)

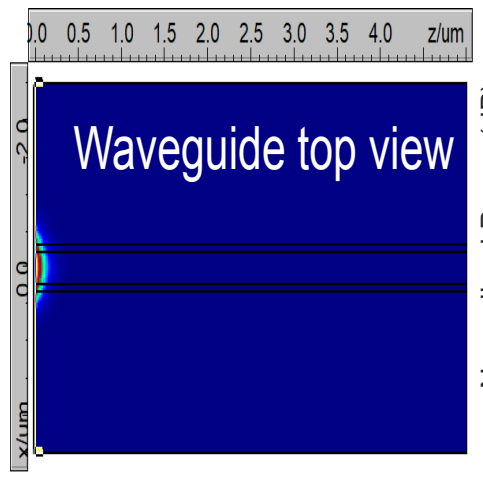

(b)

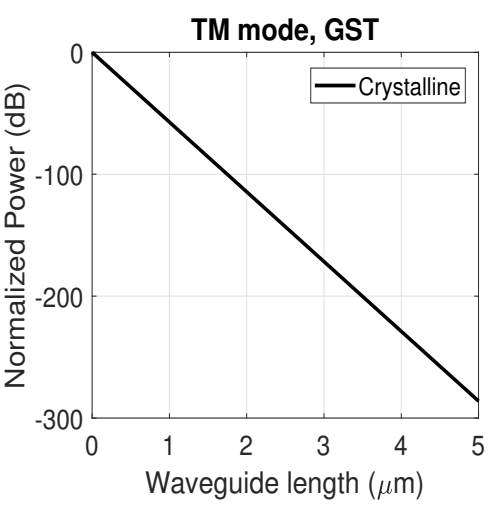

(c)

Figure 5.14: Crystalline phase a) Intensity profile of TM mode, b) Intensity distribution along the waveguide, and c) Output power of TM mode as a function of the length of the waveguide.

In addition, calculation of extinction ratio shows that a high ratio of $125 \mathrm{~dB}$ is achieved within a $3 \mu \mathrm{m}$ long waveguide.

\subsubsection{Design 4 (ChG misaligned and covering surface and one side of silicon waveguide)}

Since we are also interested in developing printed structures using ChG inks, some misalignment can lead to partial coverage of inks on the top surface and on one 
Table 5.13: Optimum design parameters obtained for Design 4.

\begin{tabular}{cc}
\hline Parameters & Value $(\mu m)$ \\
\hline $\mathrm{W}$ & 0.45 \\
\hline $\mathrm{W}_{C h G}$ & 0.3 \\
\hline $\mathrm{h}_{s i}$ & 0.22 \\
\hline $\mathrm{h}_{C h G}$ & 0.3 \\
\hline $\mathrm{h}_{O x}$ & 0.5 \\
\hline $\mathrm{h}_{b o x}$ & 2 \\
\hline $\mathrm{L}$ & 5 \\
\hline
\end{tabular}

sidewall. We also simulated such a scenario. The cross section view of the design considered is shown in Figure. 5.15. The simulated dimensions of the structure is depicted in Table 5.13.

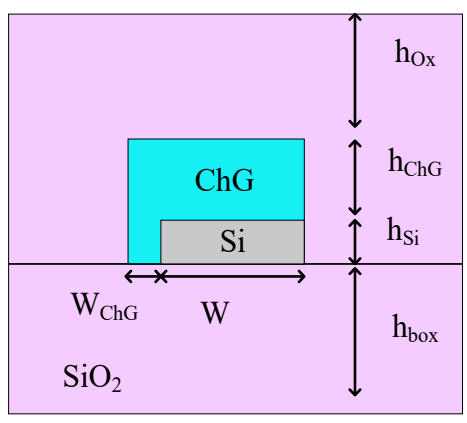

(a)

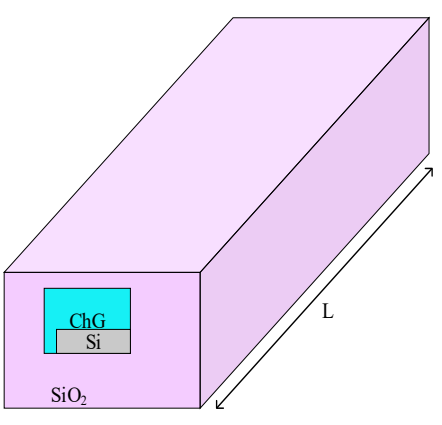

(b)

Figure 5.15: a) Cross section of design 4, b) 3D view of design 4 .

When ChG is below crystallization temperature, it acts like a dielectric. So, in TE fundamental mode, light is still concentrated in the Si waveguide, as shown in Figure. 5.16a. At the output, we will observe low loss, as is evident from Figure. 5.16b and Figure. 5.16c. Similarly, the TM mode propagates with little loss as well, as shown in Figure. 5.17. Waveguide loss in TM mode is summarized at Table 5.14. When ChG reaches its crystallization temperature, its behavior changes to metal. So, the waveguide changes to a plasmonic waveguide. Since surface plasmons can work well below the diffraction limit, the TE mode interacts strongly with the sidewall 


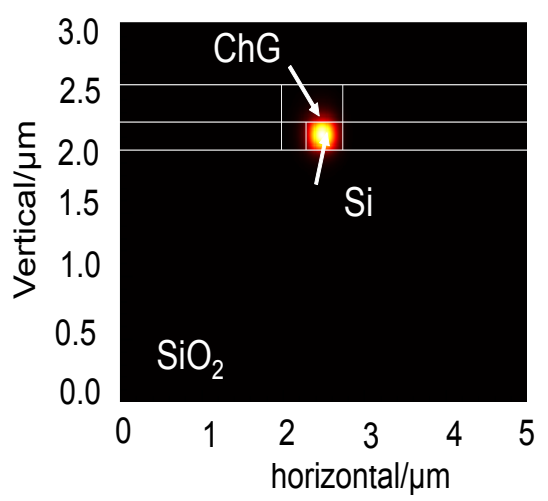

(a)

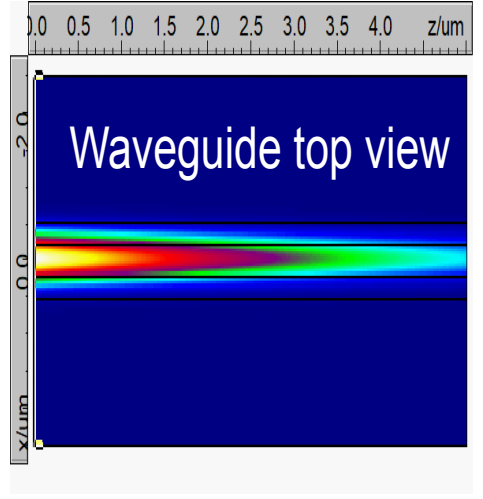

(b)

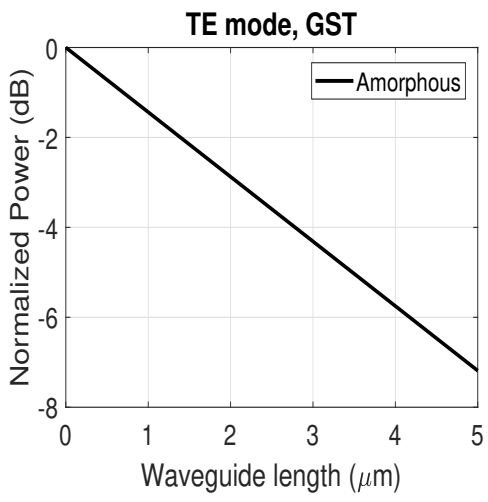

(c)

Figure 5.16: Amorphous phase a) Intensity profile of TE mode, b) Intensity distribution along the waveguide, and c) Output power of TE mode as a function of the length of the waveguide.

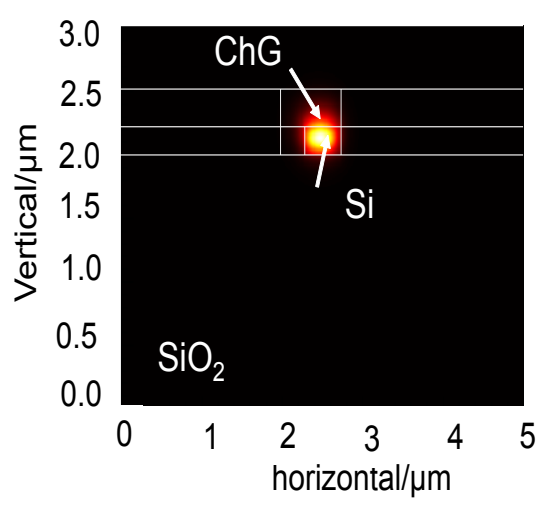

(a)

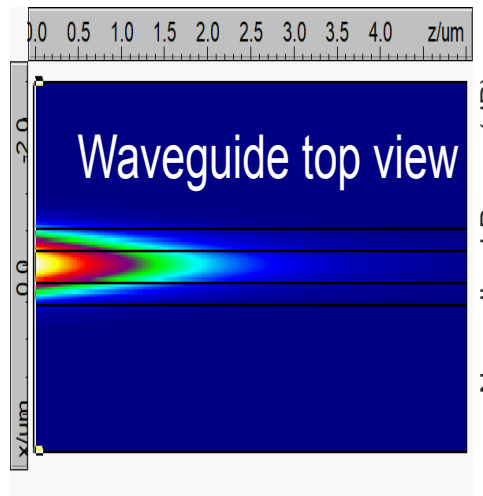

(b)

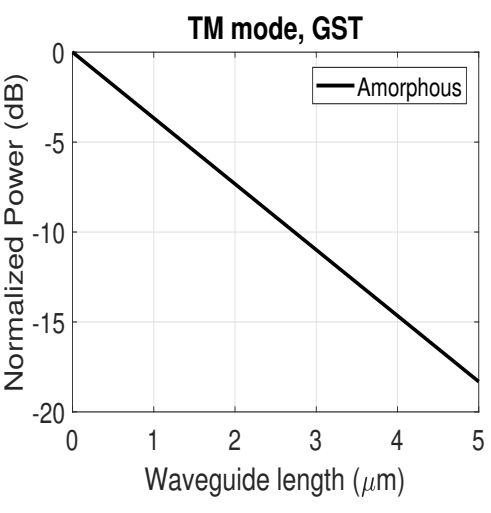

(c)

Figure 5.17: Amorphous phase a) Intensity profile of TM mode, b) Intensity distribution along the waveguide, and c) Output power of TM mode as a function of the length of the waveguide.

Table 5.14: Amorphous phase waveguide loss of Design 4.

\begin{tabular}{cccc}
\hline & $\begin{array}{c}\text { Length } \\
(\mu \mathrm{m})\end{array}$ & $\begin{array}{c}\text { Transmission loss } \\
(\mathrm{dB})\end{array}$ & $\begin{array}{c}\text { Propagation loss } \\
(\mathrm{dB} / \mu \mathrm{m})\end{array}$ \\
\hline Fundamental TE mode & 3 & -3.67 & -1.22 \\
\hline Fundamental TM mode & 3 & -10.74 & -3.58 \\
\hline
\end{tabular}




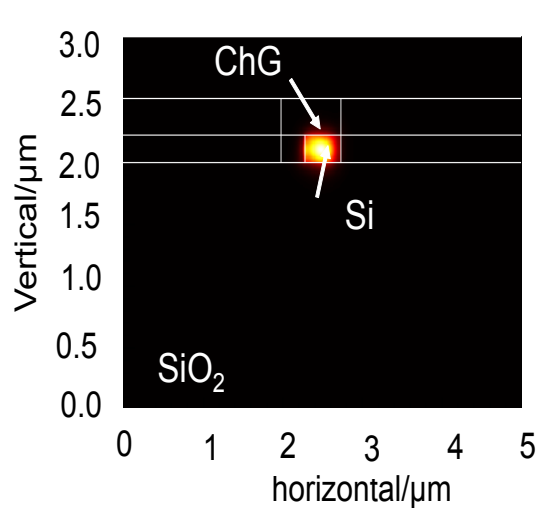

(a)

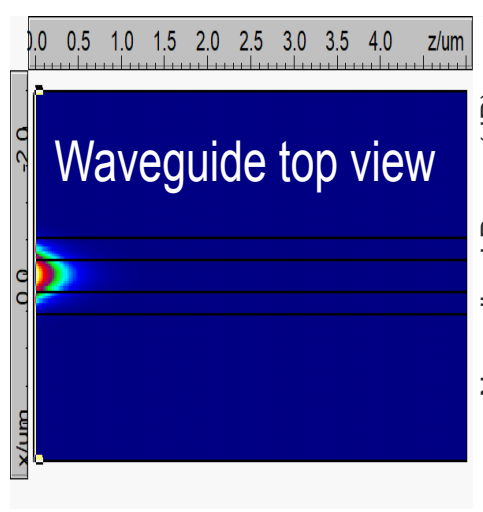

(b)

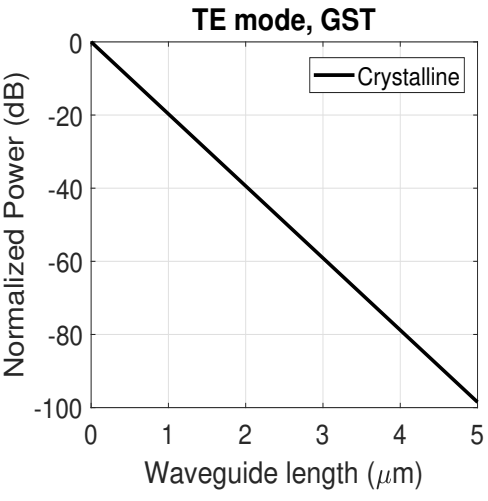

(c)

Figure 5.18: Crystalline phase a) Intensity profile of TE mode, b) Intensity distribution along the waveguide, and c) Output power of TE mode as a function of the length of the waveguide.

Table 5.15: Waveguide loss of Design 4.

\begin{tabular}{cccc} 
& $\begin{array}{c}\text { Length } \\
(\mu \mathrm{m})\end{array}$ & $\begin{array}{c}\text { Transmission loss } \\
(\mathrm{dB})\end{array}$ & $\begin{array}{c}\text { Propagation loss } \\
(\mathrm{dB} / \mu \mathrm{m})\end{array}$ \\
\hline Fundamental TM mode (amorphous) & 3 & -32.3 & -10.76 \\
\hline Fundamental TM mode (crystallization) & 3 & -198.28 & -66.09 \\
\hline
\end{tabular}

metal and is squeezed into the interface between silicon and metal, and its power diminishes rapidly (Figure. 5.18). TM mode also experiences a plasmonic effect, and its power decreases as well (Figure. 5.19). Depending on the degree to which the ChG covers the top surface, a plasmonic mode will still be generated, again owing to the ability of plasmonic modes to be confined well below the diffraction limit, and a very high loss can be expected due to the subwavelength confinement property of plasmonic modes. Thus, even if the silicon waveguide is partially covered with ChG, the device can still perform efficiently. Waveguide loss in TM mode is summarized at Table 5.15. Compared to Design \#1, wherein the ChG fully covers the silicon waveguide, the misaligned case represented by Design \#4 produces lower loss, however, an 


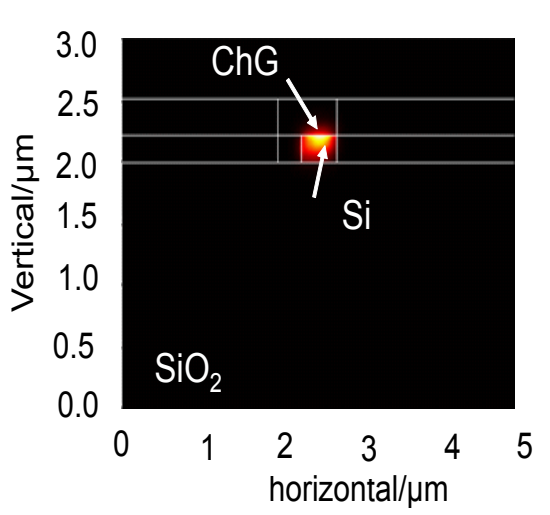

(a)

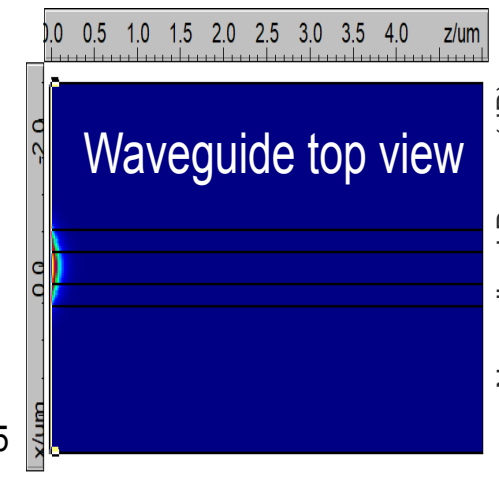

(b)

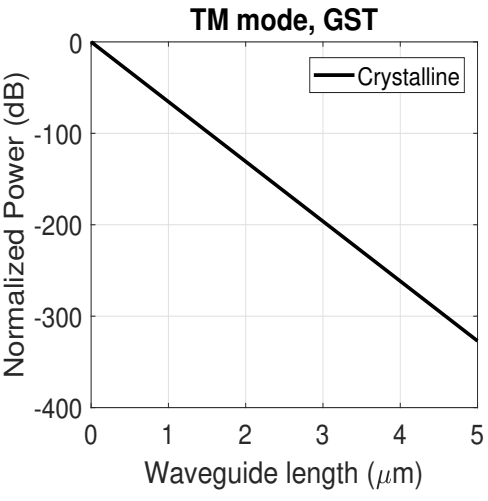

(c)

Figure 5.19: Crystalline phase a) Intensity profile of TM mode, b) Intensity distribution along the waveguide, and c) Output power of TM mode as a function of the length of the waveguide.

Table 5.16: Performance comparison between the 4 design architectures.

\begin{tabular}{|c|c|c|c|c|}
\hline & & Amorphous & & Crystalline \\
\hline & $\begin{array}{l}\text { Loss in TE mode } \\
\qquad(\mathrm{dB} / \mu \mathrm{m})\end{array}$ & $\begin{array}{l}\text { Loss in TM mode } \\
(\mathrm{dB} / \mu \mathrm{m})\end{array}$ & $\begin{array}{l}\text { Loss in TE mode } \\
(\mathrm{dB} / \mu \mathrm{m})\end{array}$ & $\begin{array}{l}\text { Loss in TM mode } \\
(\mathrm{dB} / \mu \mathrm{m})\end{array}$ \\
\hline Design 1 & -5.46 & -11.34 & -95.05 & -207.73 \\
\hline Design 2 & -2.15 & -4.63 & -25.36 & -200.84 \\
\hline Design 3 & -2.33 & -9.75 & -28.69 & -155.91 \\
\hline Design 4 & -3.67 & -10.74 & -32.3 & -198.28 \\
\hline
\end{tabular}

extinction ratio $>10 \mathrm{~dB}$ is still available for successful device operation. Comparison of all waveguide design losses in fundamental TE and TM mode in amorphous and crystalline phases of ChG is shown in Table 5.16. 


\subsection{Design of Plasmonic based Temperature Sensor Using Characterization Data from In-House Synthesized Inks}

The same designs are simulated with six Ge-S and Ge-Se chalcogenide glasses synthesized at Boise State University. The complex refractive index is measured using an ellipsometer [93]. The refractive indices and extinction coefficients of all compositions in two amorphous and crystalline phases are shown in Table 3.1. We have simulated Designs 1, 3 and, 4 with the measured refractive index of synthesized inks using the same dimensions in both phases of chalcogenide glass.

\subsubsection{Design 1 (in-house synthesized inks covering Silicon Waveg- uide)}

In this structure, Si waveguide is surrounded by six different in-house synthesized inks. The ChG is covered by a passivation layer, which is $\mathrm{SiO}_{2}$ in our case. The simulation results for fundamental TM mode for Design 1 are shown in Figure. 5.20 through Figure. 5.31 for all six Ge-S and Ge-Se compositions in amorphous and crystalline phases. As expected, in the amorphous phase of ink, fundamental TM mode is confined within the $\mathrm{Si}$ core. It is clearly shown that the incident wave can easily pass through the $\mathrm{Si}$ core without significant attenuation, when the $\mathrm{ChG}$ cap is in the amorphous phase. At crystallization temperature of $\mathrm{ChG}$, the phase of the glass is changed to metal phase. In this case, plasmonic waveguide is created. The

device has higher loss in crystalline phase compared to amorphous phase of Ge-S and Ge-Se. The attenuation of the SPP significantly increases, so that SPP mode cannot 
be transmitted to the output. Various transmitted output power is due to changes in refractive index of the ChG by the phase transition from amorphous to crystalline. Waveguide loss in TM mode is summarized at Table 5.17.

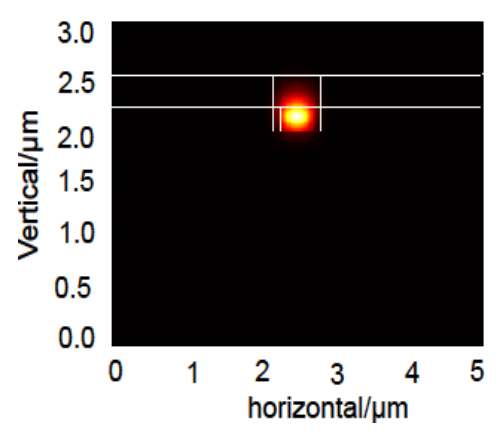

(a)

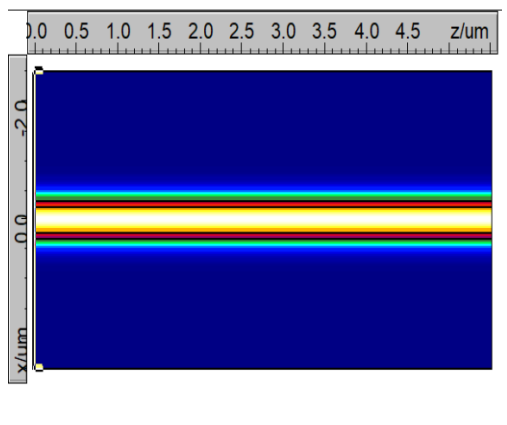

(b)

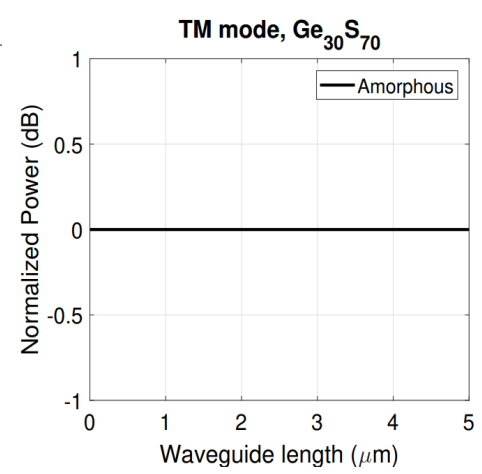

(c)

Figure 5.20: Amorphous $\mathrm{Ge}_{30} \mathrm{~S}_{70}$ patterned on the top surface of silicon waveguide in crystalline phase. a) Intensity profile of TM mode, b) Intensity distribution along the waveguide, and c) Output power of TM mode as a function of the length of the waveguide.

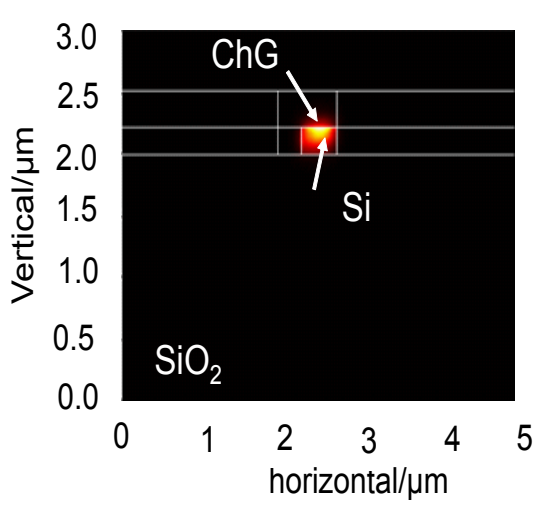

(a)

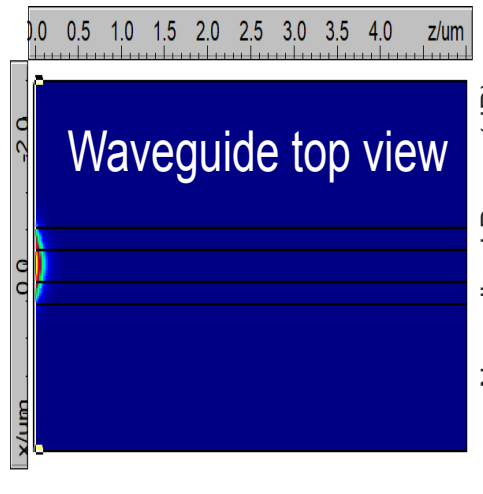

(b)

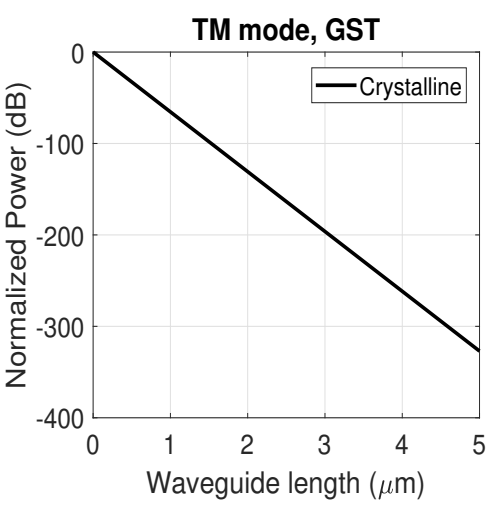

(c)

Figure 5.21: Crystalline $\mathrm{Ge}_{30} \mathrm{~S}_{70}$ patterned on the top surface of silicon waveguide in crystalline phase. a) Intensity profile of TM mode, b) Intensity distribution along the waveguide, and c) Output power of TM mode as a function of the length of the waveguide. 


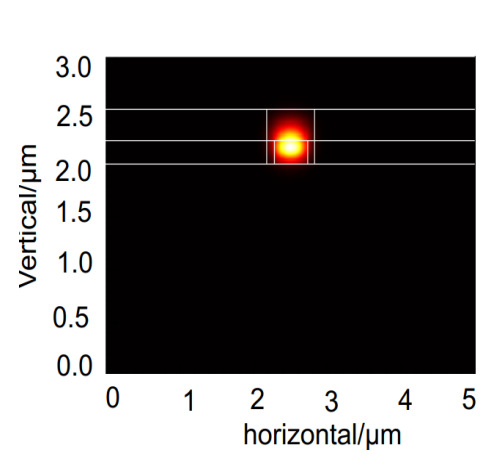

(a)

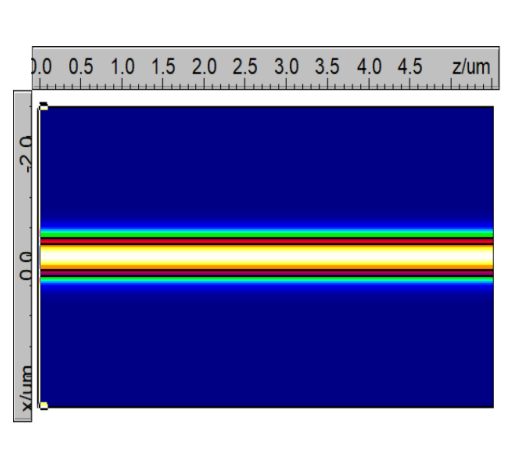

(b)

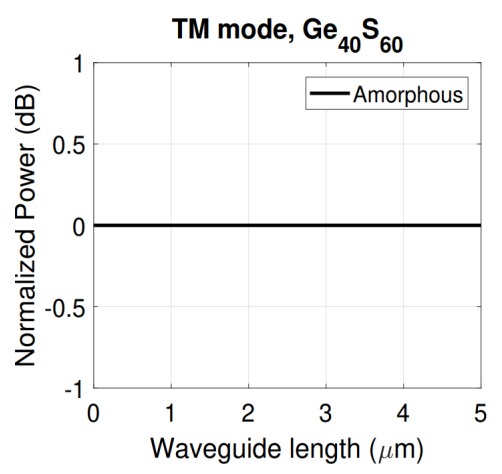

(c)

Figure 5.22: Amorphous $\mathrm{Ge}_{40} \mathrm{~S}_{60}$ patterned on the top surface of silicon waveguide in crystalline phase. a) Intensity profile of TM mode, b) Intensity distribution along the waveguide, and c) Output power of TM mode as a function of the length of the waveguide.

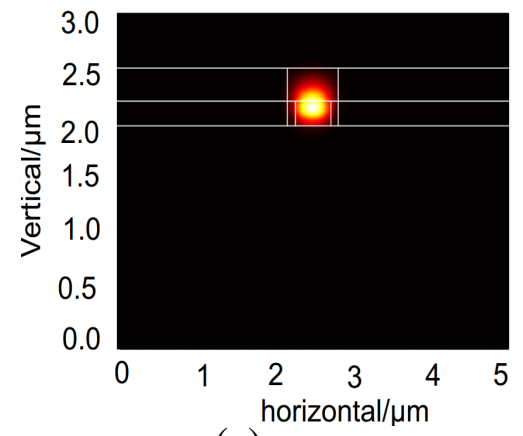

(a)

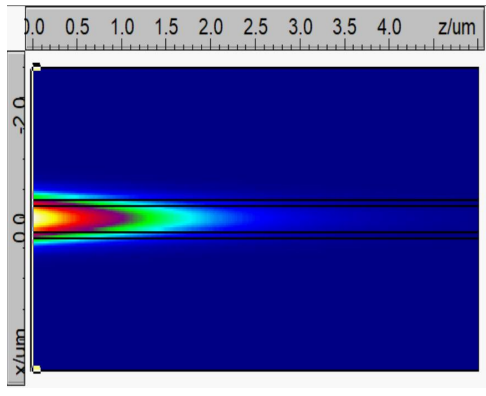

(b)

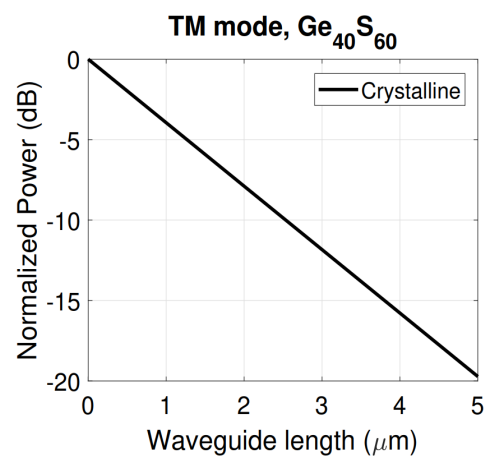

(c)

Figure 5.23: Crystalline $\mathrm{Ge}_{40} \mathrm{~S}_{60}$ patterned on the top surface of silicon waveguide in crystalline phase. a) Intensity profile of TM mode, b) Intensity distribution along the waveguide, and c) Output power of TM mode as a function of the length of the waveguide. 


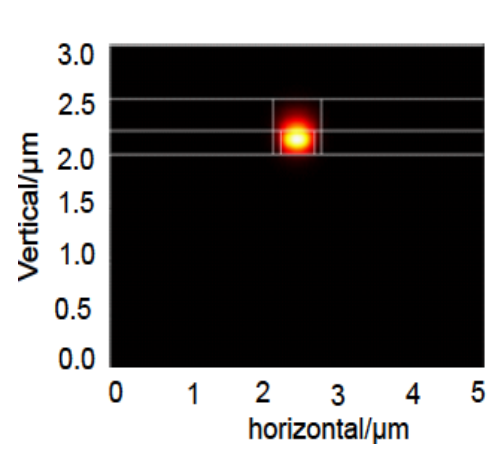

(a)

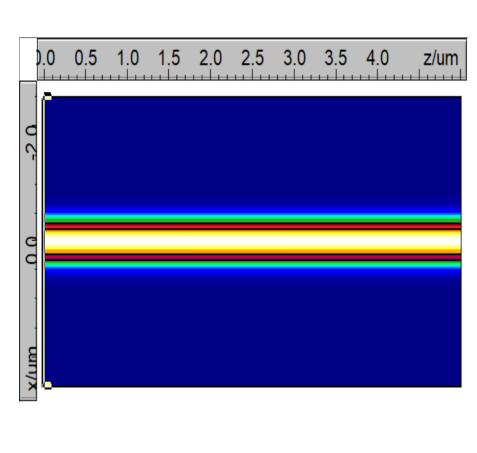

(b)

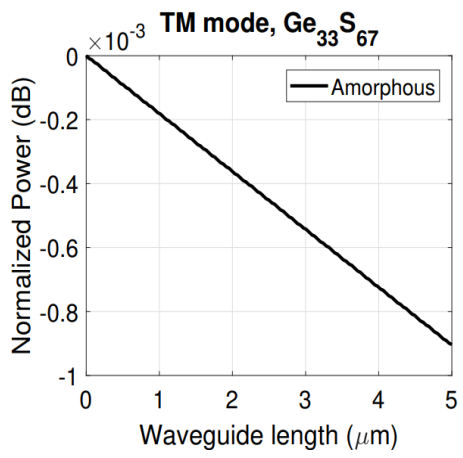

(c)

Figure 5.24: Amorphous $\mathrm{Ge}_{33} \mathrm{~S}_{67}$ patterned on the top surface of silicon waveguide in crystalline phase. a) Intensity profile of TM mode, b) Intensity distribution along the waveguide, and c) Output power of TM mode as a function of the length of the waveguide.

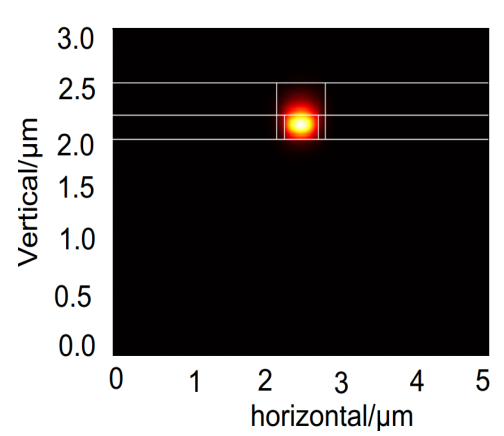

(a)

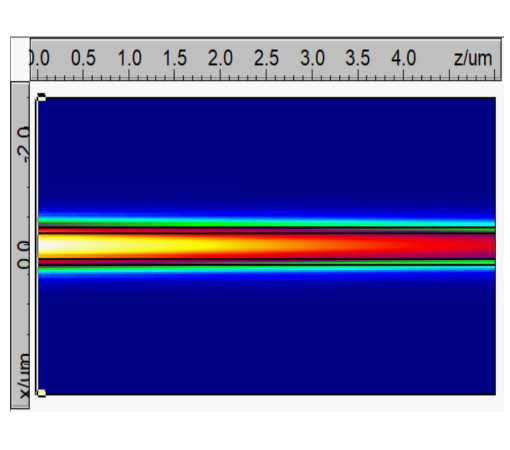

(b)

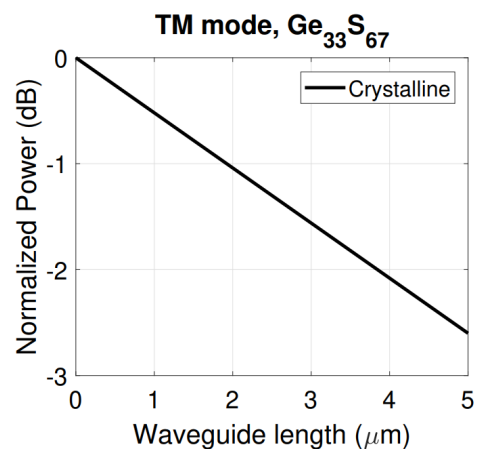

(c)

Figure 5.25: Crystalline $\mathrm{Ge}_{33} \mathrm{~S}_{67}$ patterned on the top surface of silicon waveguide in crystalline phase. a) Intensity profile of TM mode, b) Intensity distribution along the waveguide, and c) Output power of TM mode as a function of the length of the waveguide. 


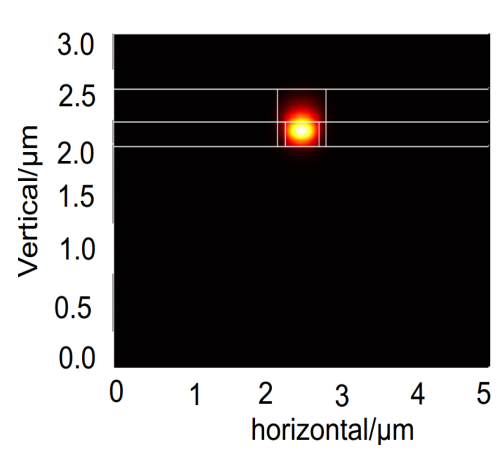

(a)

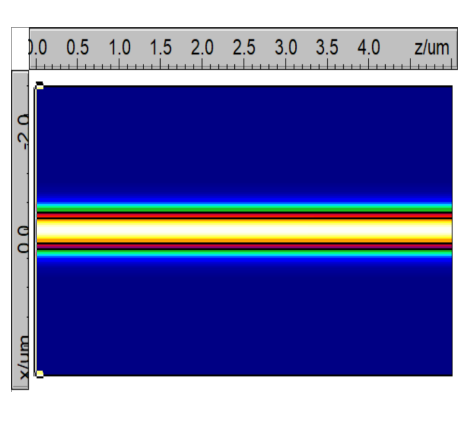

(b)

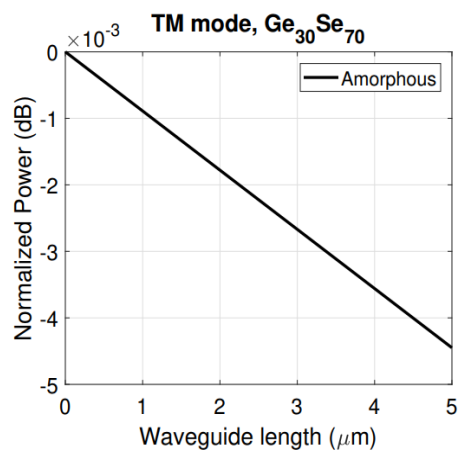

(c)

Figure 5.26: Amorphous $\mathrm{Ge}_{30} \mathrm{Se}_{70}$ patterned on the top surface of silicon waveguide in crystalline phase. a) Intensity profile of TM mode, b) Intensity distribution along the waveguide, and c) Output power of TM mode as a function of the length of the waveguide.

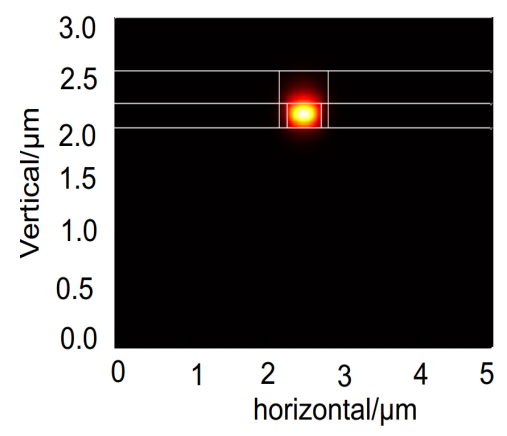

(a)

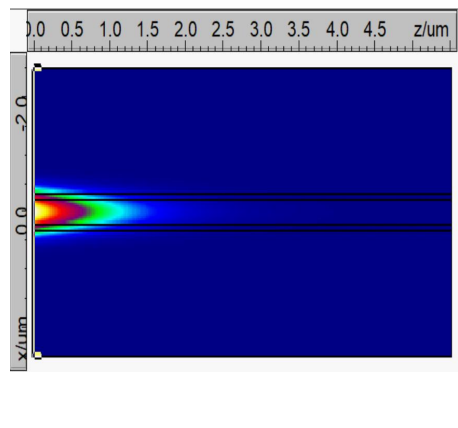

(b)

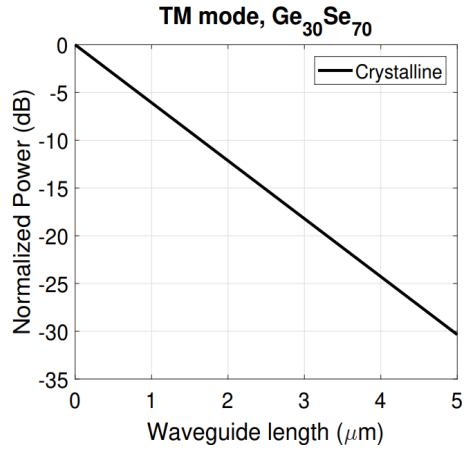

(c)

Figure 5.27: Crystalline $\mathrm{Ge}_{30} \mathrm{Se}_{70}$ patterned on the top surface of silicon waveguide in crystalline phase. a) Intensity profile of TM mode, b) Intensity distribution along the waveguide, and c) Output power of TM mode as a function of the length of the waveguide. 


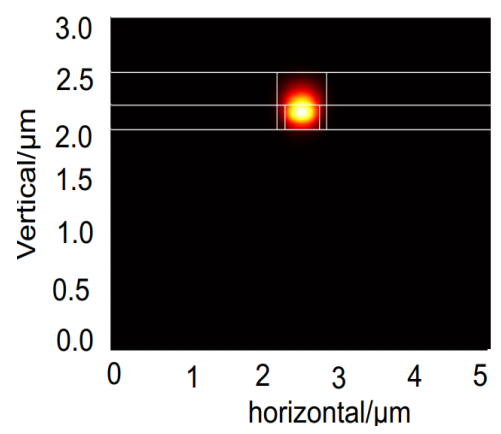

(a)

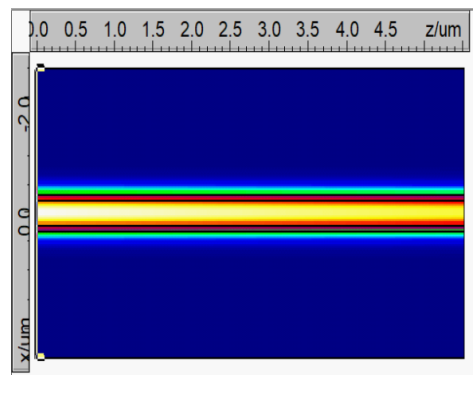

(b)

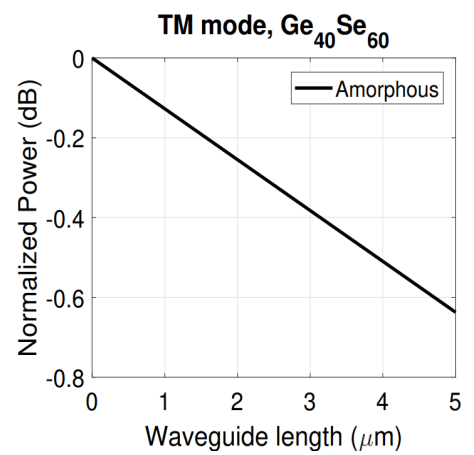

(c)

Figure 5.28: Amorphous $\mathrm{Ge}_{40} \mathrm{Se}_{60}$ patterned on the top surface of silicon waveguide in crystalline phase. a) Intensity profile of TM mode, b) Intensity distribution along the waveguide, and c) Output power of TM mode as a function of the length of the waveguide.

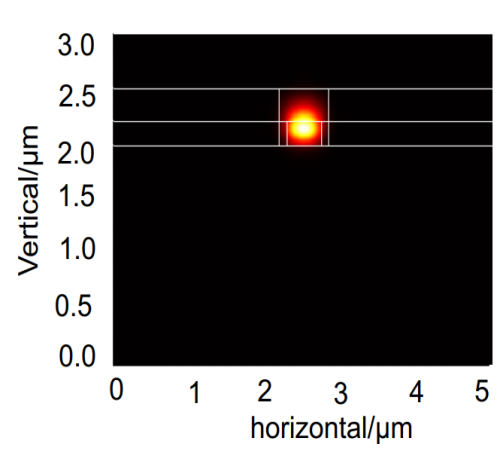

(a)

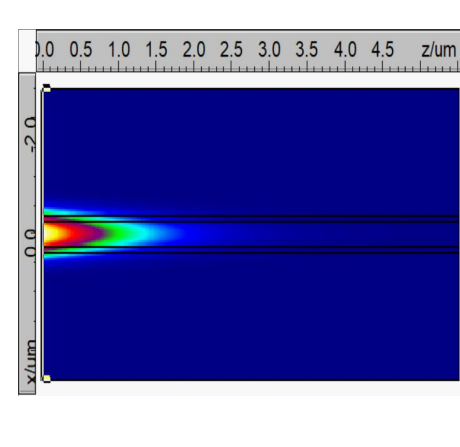

(b)

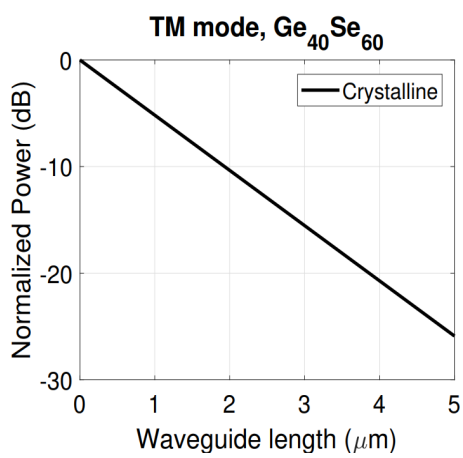

(c)

Figure 5.29: Crystalline $\mathrm{Ge}_{40} \mathrm{Se}_{60}$ patterned on the top surface of silicon waveguide in crystalline phase. a) Intensity profile of TM mode, b) Intensity distribution along the waveguide, and c) Output power of TM mode as a function of the length of the waveguide. 


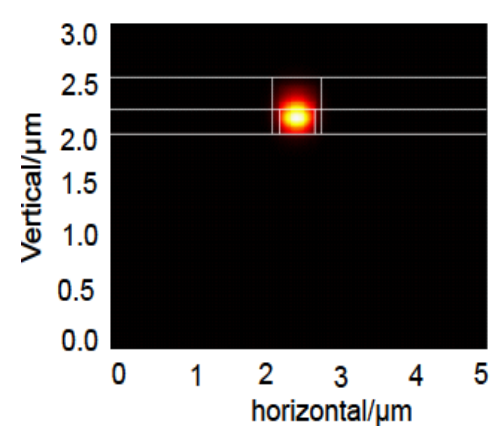

(a)

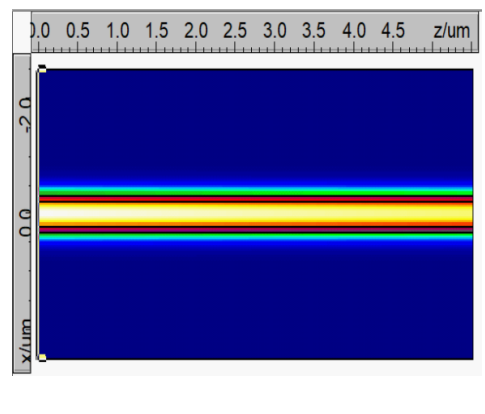

(b)

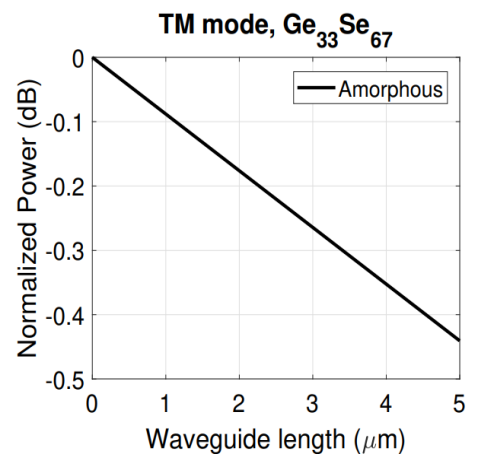

(c)

Figure 5.30: Amorphous $\mathrm{Ge}_{33} \mathrm{Se}_{67}$ patterned on the top surface of silicon waveguide in crystalline phase. a) Intensity profile of TM mode, b) Intensity distribution along the waveguide, and c) Output power of TM mode as a function of the length of the waveguide.

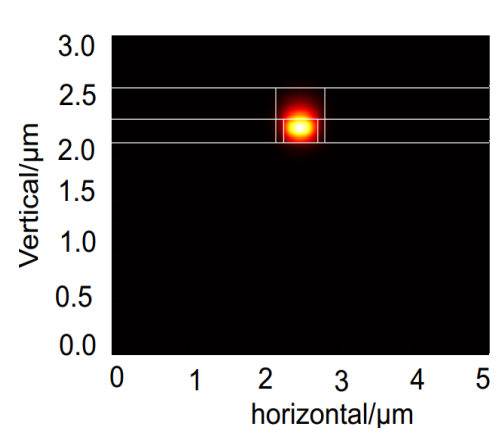

(a)

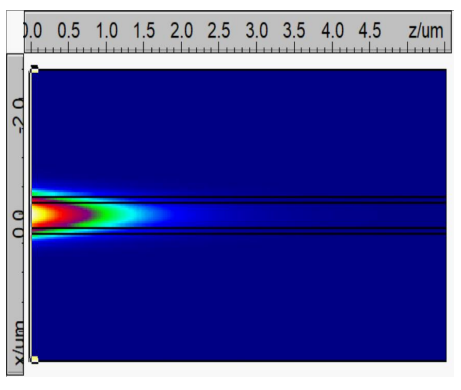

(b)

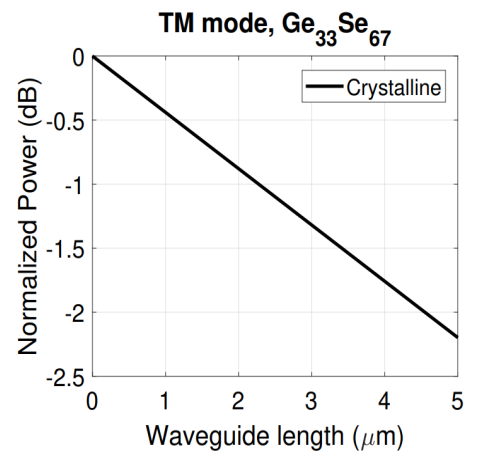

(c)

Figure 5.31: Crystalline $\mathrm{Ge}_{33} \mathrm{Se}_{67}$ patterned on the top surface of silicon waveguide in crystalline phase. a) Intensity profile of TM mode, b) Intensity distribution along the waveguide, and c) Output power of TM mode as a function of the length of the waveguide. 
Table 5.17: Fundamental TM mode loss in Waveguide design 1 with inhouse synthesized in-house inks

\begin{tabular}{cccc}
\hline Length & $\begin{array}{c}\text { Propagation loss } \\
\text { in amorphous phase } \\
(\mathrm{dB} / \mu m)\end{array}$ & $\begin{array}{c}\text { Propagation loss } \\
\text { in crystalline phase } \\
(\mathrm{dB} / \mu m)\end{array}$ \\
\hline $\mathrm{Ge}_{30} \mathrm{~S}_{70}$ & 3 & 0 & -2.42 \\
\hline $\mathrm{Ge}_{40} \mathrm{~S}_{60}$ & 3 & 0 & -3.95 \\
\hline $\mathrm{Ge}_{33} \mathrm{~S}_{67}$ & 3 & -0.08 & -0.44 \\
\hline $\mathrm{Ge}_{30} \mathrm{Se}_{70}$ & 3 & 0 & -6.08 \\
\hline $\mathrm{Ge}_{40} \mathrm{Se}_{60}$ & 3 & -0.12 & -5.19 \\
\hline $\mathrm{Ge}_{33} \mathrm{Se}_{67}$ & 3 & -0.08 & -0.44 \\
\hline
\end{tabular}

\subsubsection{Design 3 (in-house synthesized inks on the top surface of Silicon Waveguide)}

In this structure, Si waveguide top surface is covered with six different in-house synthesized inks. The simulation results for fundamental TM mode for Design 3 are shown in Figure. 5.32 through Figure. 5.43 for all six Ge-S and Ge-Se compositions in amorphous and crystalline phases. Waveguide loss in TM mode is summarized in Table 5.18. 


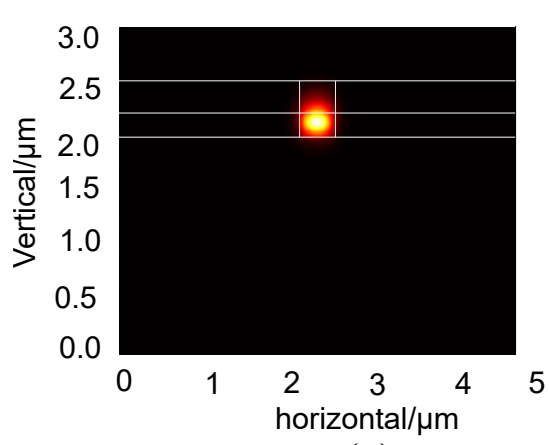

(a)

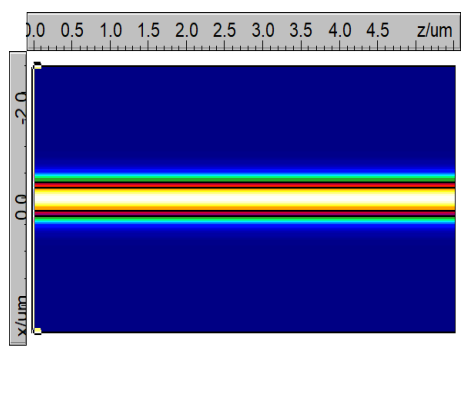

(b)

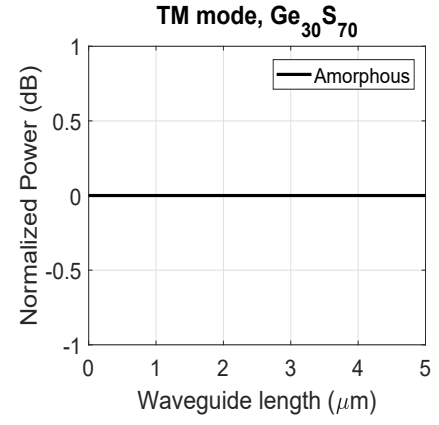

(c)

Figure 5.32: Amorphous $\mathrm{Ge}_{30} \mathrm{~S}_{70}$ patterned on the top surface of silicon waveguide in amorphous phase. a) Intensity profile of TM mode, b) Intensity distribution along the waveguide, and c) Output power of TM mode as a function of the length of the waveguide.

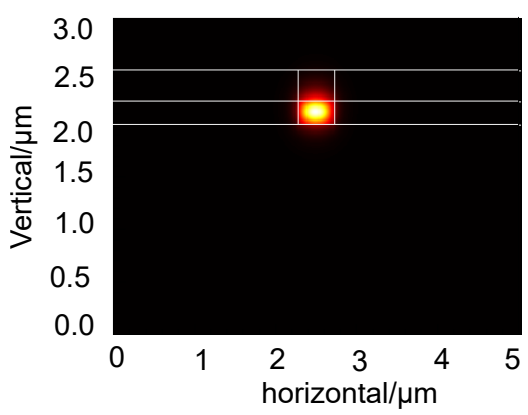

(a)

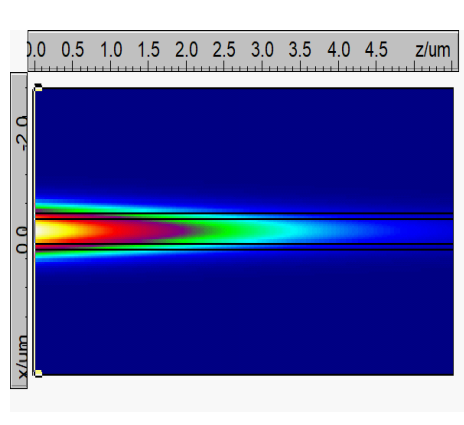

(b)

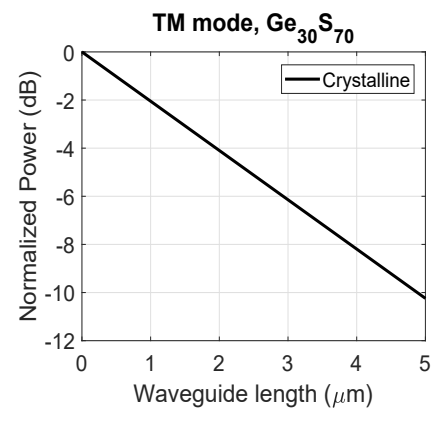

(c)

Figure 5.33: Crystalline $\mathrm{Ge}_{30} \mathrm{~S}_{70}$ patterned on the top surface of silicon waveguide in crystalline phase. a) Intensity profile of TM mode, b) Intensity distribution along the waveguide, and c) Output power of TM mode as a function of the length of the waveguide. 


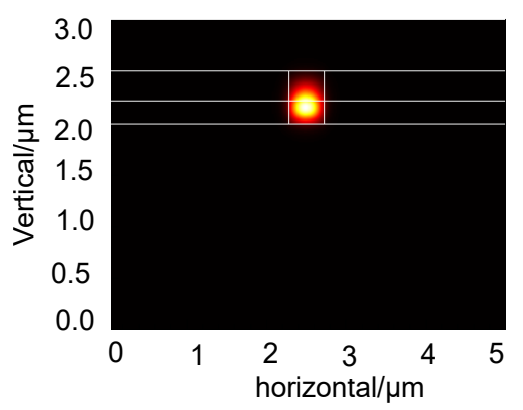

(a)

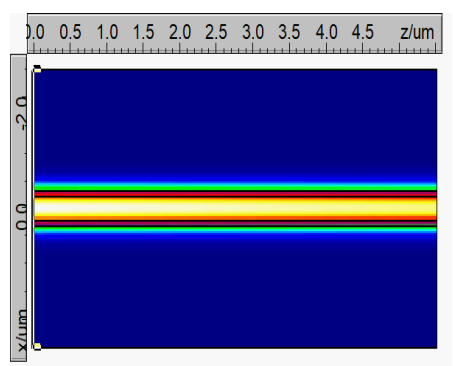

(b)

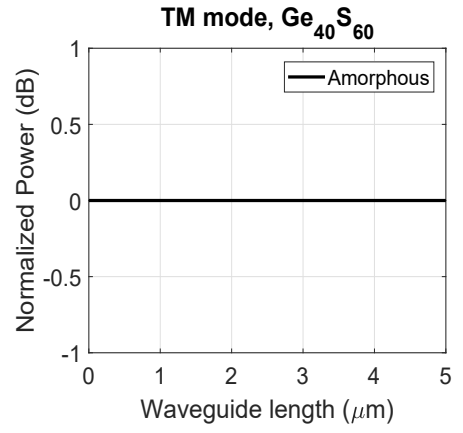

(c)

Figure 5.34: Amorphous $\mathrm{Ge}_{40} \mathrm{~S}_{60}$ patterned on the top surface of silicon waveguide in amorphous phase. a) Intensity profile of TM mode, b) Intensity distribution along the waveguide, and c) Output power of TM mode as a function of the length of the waveguide.

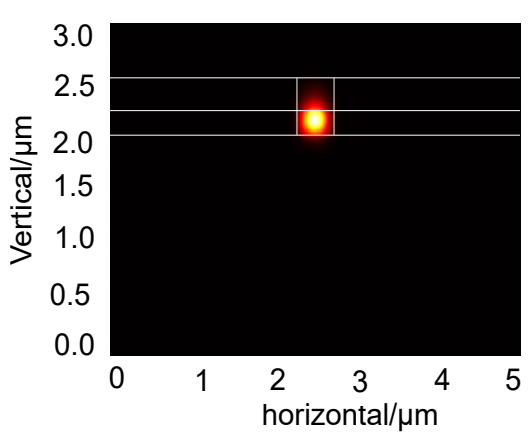

(a)

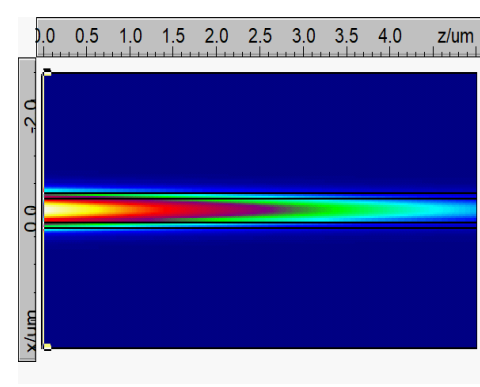

(b)

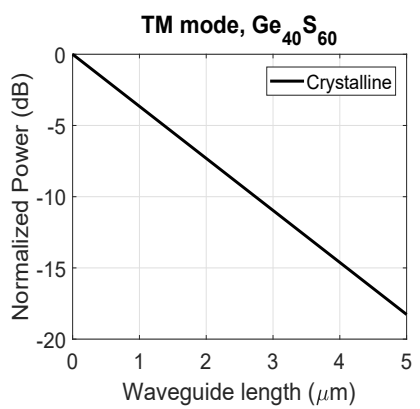

(c)

Figure 5.35: Crystalline $\mathrm{Ge}_{40} \mathrm{~S}_{60}$ patterned on the top surface of silicon waveguide in crystalline phase. a) Intensity profile of TM mode, b) Intensity distribution along the waveguide, and c) Output power of TM mode as a function of the length of the waveguide. 


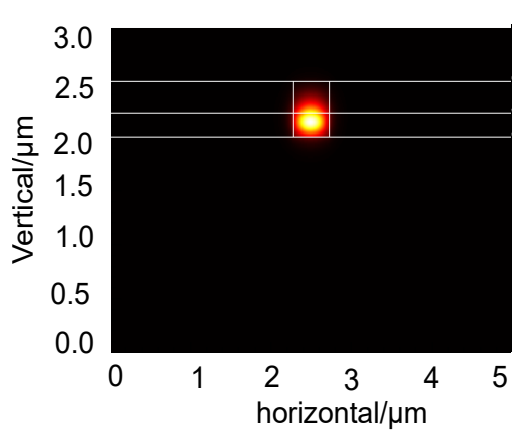

(a)

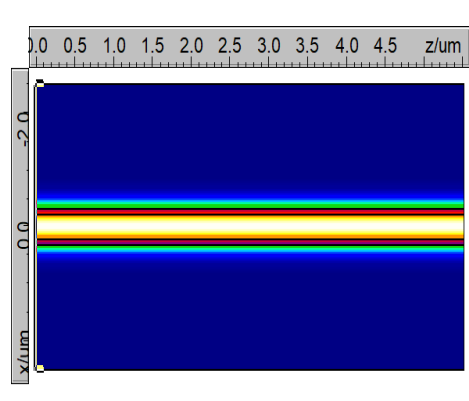

(b)

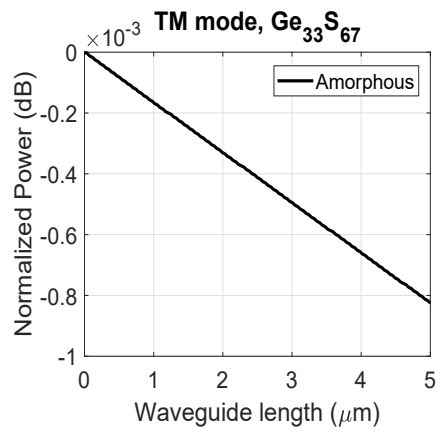

(c)

Figure 5.36: Amorphous $\mathrm{Ge}_{33} \mathrm{~S}_{67}$ patterned on the top surface of silicon waveguide in amorphous phase. a) Intensity profile of TM mode, b) Intensity distribution along the waveguide, and c) Output power of TM mode as a function of the length of the waveguide.

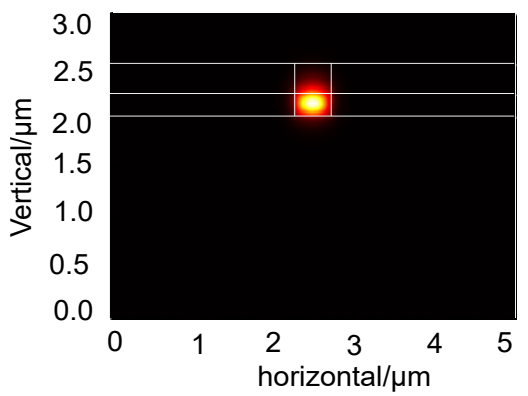

(a)

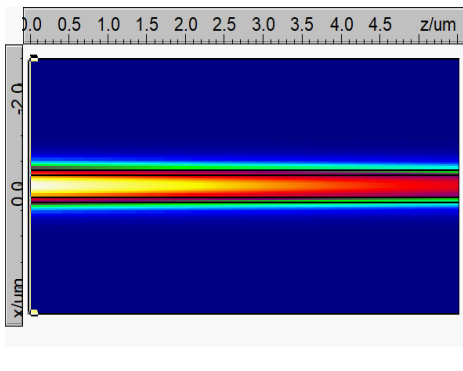

(b)

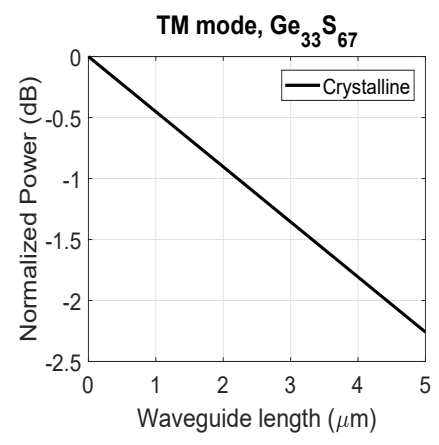

(c)

Figure 5.37: Crystalline $\mathrm{Ge}_{33} \mathrm{~S}_{67}$ patterned on the top surface of silicon waveguide in crystalline phase. a) Intensity profile of TM mode, b) Intensity distribution along the waveguide, and c) Output power of TM mode as a function of the length of the waveguide. 


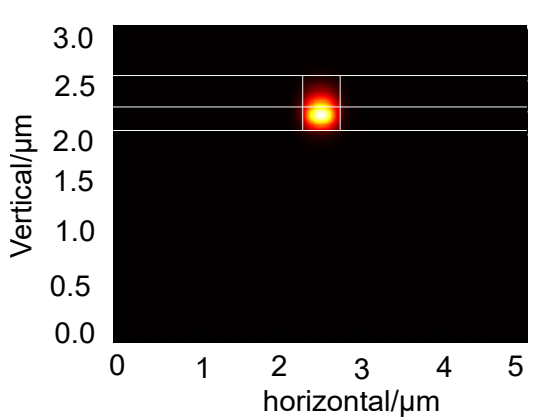

(a)

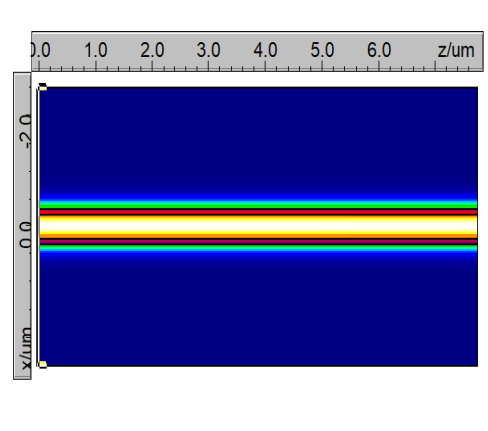

(b)

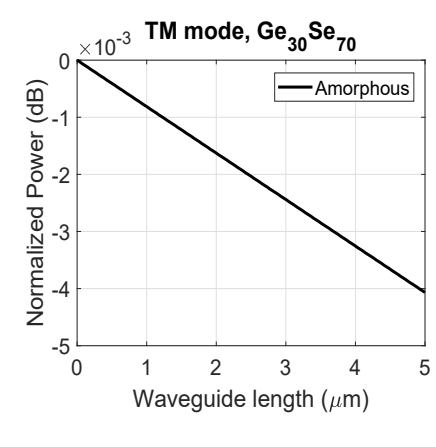

(c)

Figure 5.38: Amorphous $\mathrm{Ge}_{30} \mathrm{Se}_{70}$ patterned on the top surface of silicon waveguide in amorphous phase. a) Intensity profile of TM mode, b) Intensity distribution along the waveguide, and c) Output power of TM mode as a function of the length of the waveguide.

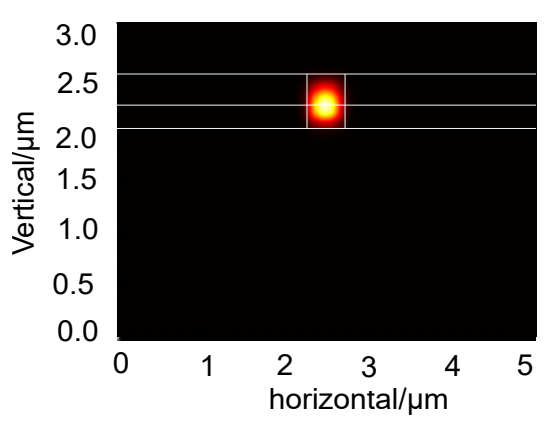

(a)

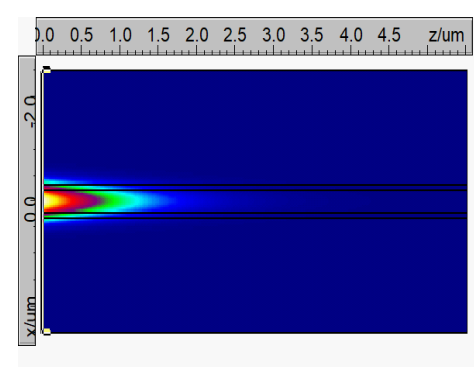

(b)

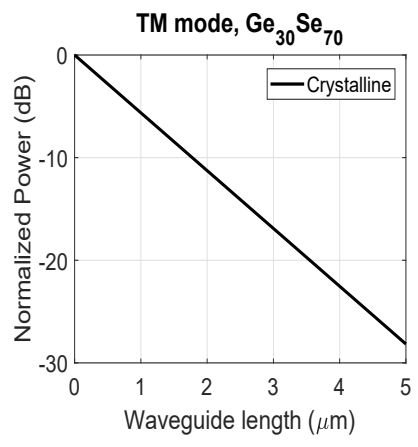

(c)

Figure 5.39: Crystalline $\mathrm{Ge}_{30} \mathrm{Se}_{70}$ patterned on the top surface of silicon waveguide in crystalline phase . a) Intensity profile of TM mode, b) Intensity distribution along the waveguide, and c) Output power of TM mode as a function of the length of the waveguide. 


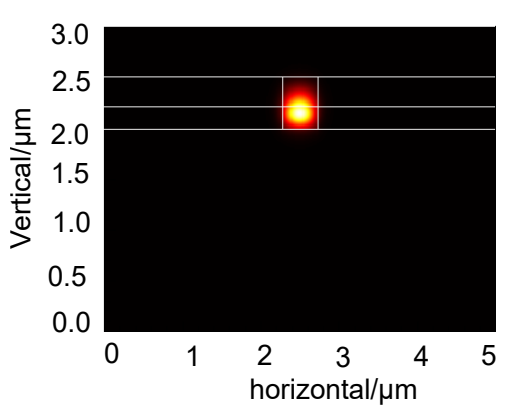

(a)

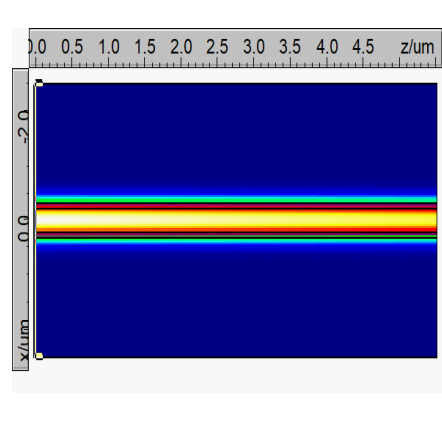

(b)

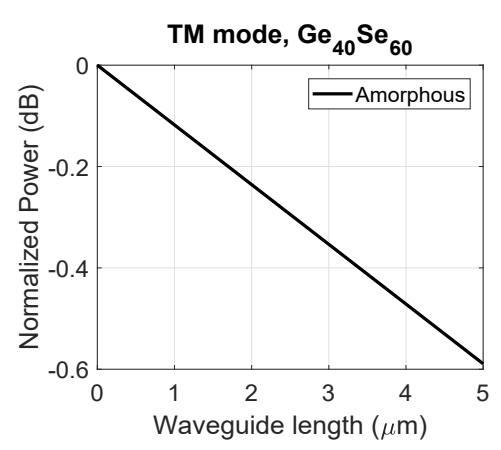

(c)

Figure 5.40: Amorphous $\mathrm{Ge}_{40} \mathrm{Se}_{60}$ patterned on the top surface of silicon waveguide in amorphous phase . a) Intensity profile of TM mode, b) Intensity distribution along the waveguide, and c) Output power of TM mode as a function of the length of the waveguide.

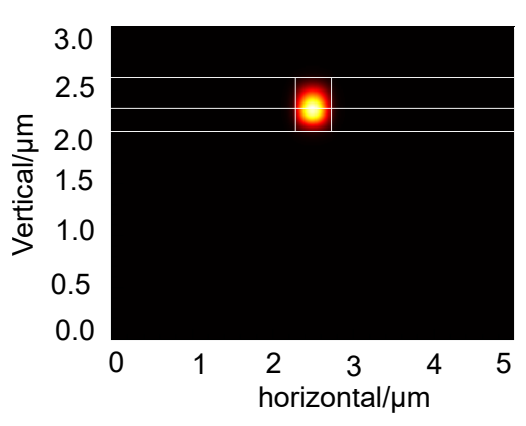

(a)

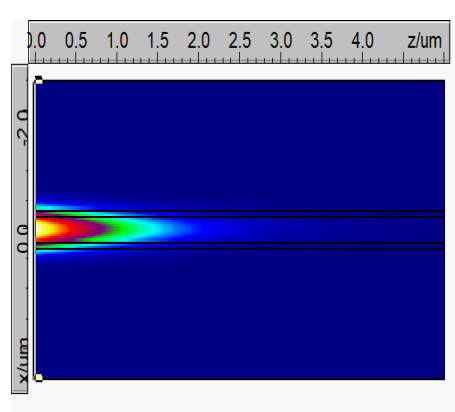

(b)

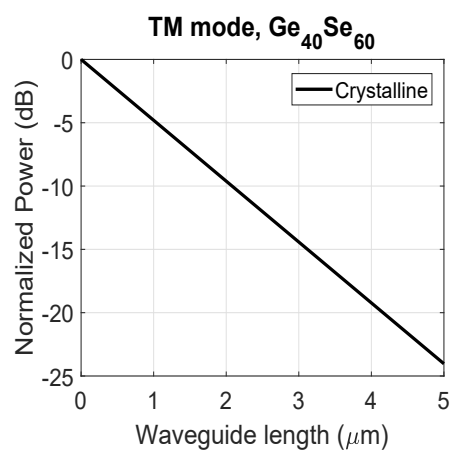

(c)

Figure 5.41: Crystalline $\mathrm{Ge}_{40} \mathrm{Se}_{60}$ patterned on the top surface of silicon waveguide in crystalline phase. a) Intensity profile of TM mode, b) Intensity distribution along the waveguide, and c) Output power of TM mode as a function of the length of the waveguide. 


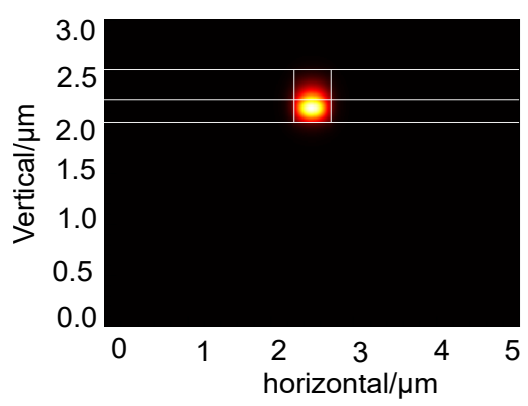

(a)

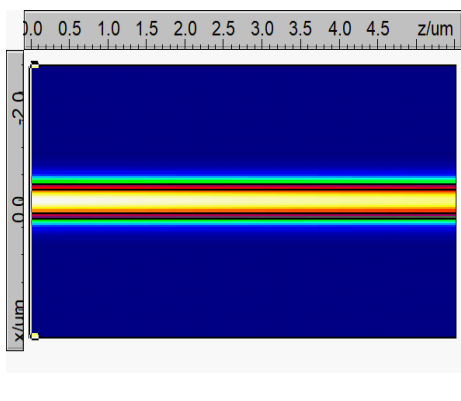

(b)

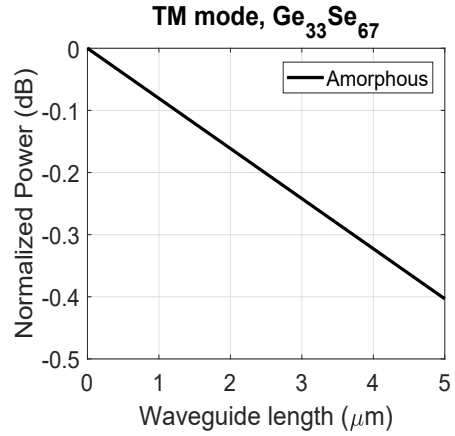

(c)

Figure 5.42: Amorphous $\mathrm{Ge}_{33} \mathrm{Se}_{67}$ patterned on the top surface of silicon waveguide in amorphous phase. a) Intensity profile of TM mode, b) Intensity distribution along the waveguide, and c) Output power of TM mode as a function of the length of the waveguide.

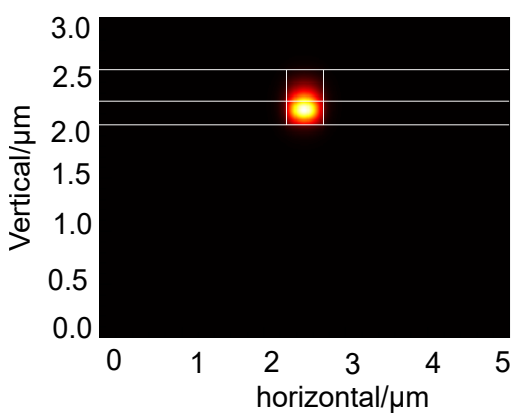

(a)

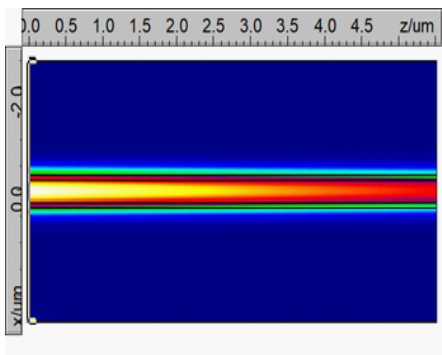

(b)

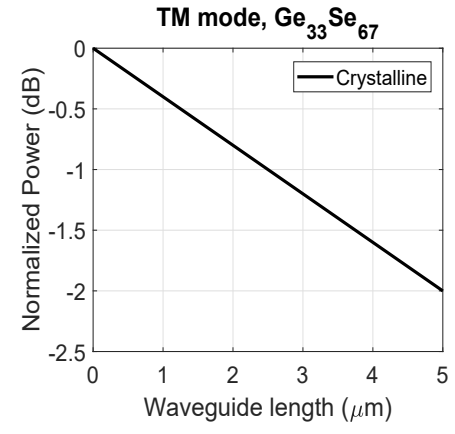

(c)

Figure 5.43: Crystalline $\mathrm{Ge}_{33} \mathrm{Se}_{67}$ patterned on the top surface of silicon waveguide in crystalline phase. a) Intensity profile of TM mode, b) Intensity distribution along the waveguide, and c) Output power of TM mode as a function of the length of the waveguide.

\subsubsection{Design 4 (in-house synthesized inks misaligned and cov- ering surface and one side of silicon waveguide)}

We also simulated such a structure in which the ink covered only the top surface and one side of the Si waveguide. We assumed that ink is misaligned during printing it on the $\mathrm{Si}$ waveguide section The simulation results for fundamental TM mode for 
Table 5.18: Fundamental TM mode loss in Waveguide design 3 with inhouse synthesized inks.

\begin{tabular}{cccc} 
Length & $\begin{array}{c}\text { Propagation loss } \\
\text { in amorphous phase } \\
(\mu \mathrm{m})\end{array}$ & $\begin{array}{c}\text { Propagation loss } \\
\text { in crystalline phase } \\
(\mathrm{dB} / \mu \mathrm{m})\end{array}$ \\
\hline $\mathrm{Ge}_{30} \mathrm{~S}_{70}$ & 3 & 0 & -2.05 \\
\hline $\mathrm{Ge}_{40} \mathrm{~S}_{60}$ & 3 & -0 & -3.66 \\
\hline $\mathrm{Ge}_{33} \mathrm{~S}_{67}$ & 3 & 0 & -0.45 \\
\hline $\mathrm{Ge}_{30} \mathrm{Se}_{70}$ & 3 & 0 & -5.64 \\
\hline $\mathrm{Ge}_{40} \mathrm{Se}_{60}$ & 3 & -0.11 & -4.81 \\
\hline $\mathrm{Ge}_{33} \mathrm{Se}_{67}$ & 3 & -0.08 & -0.44 \\
\hline
\end{tabular}

Design 4 are shown in Figure. 5.44 through Figure. 5.55 for all six Ge-S and Ge-Se compositions in amorphous and crystalline phases. Waveguide loss in TM mode is summarized at Table 5.19.

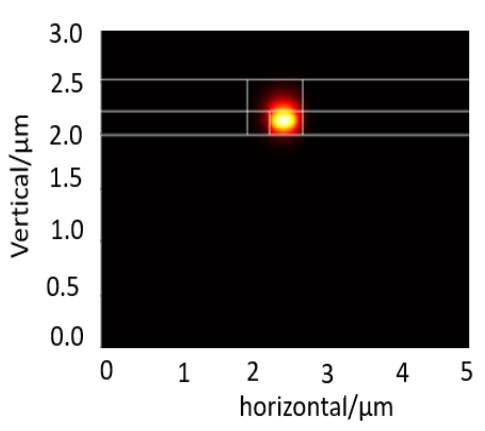

(a)

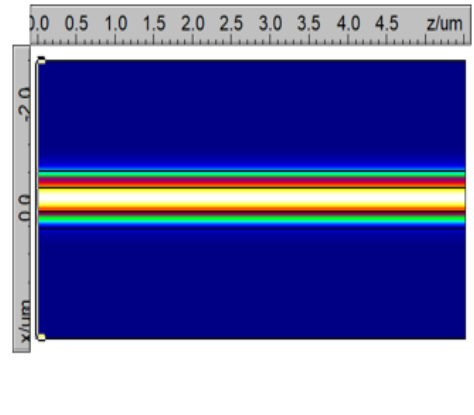

(b)

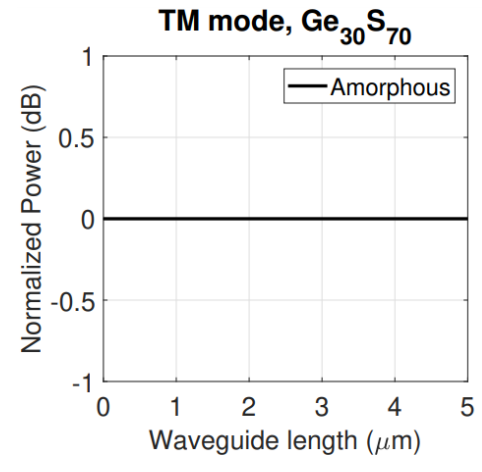

(c)

Figure 5.44: Amorphous $\mathrm{Ge}_{30} \mathrm{~S}_{70}$ patterned on the top surface of silicon waveguide in crystalline phase. a) Intensity profile of TM mode, b) Intensity distribution along the waveguide, and c) Output power of TM mode as a function of the length of the waveguide. 


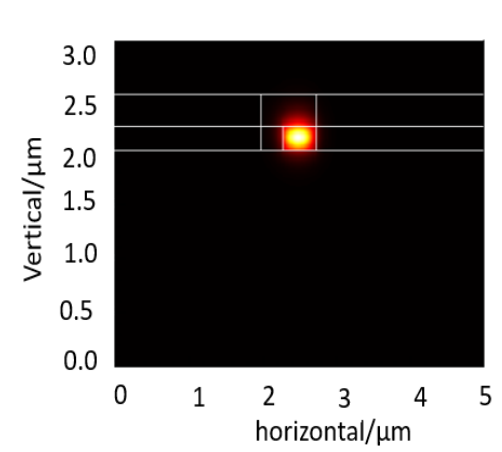

(a)

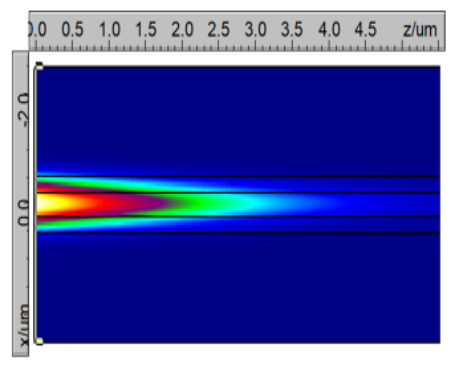

(b)

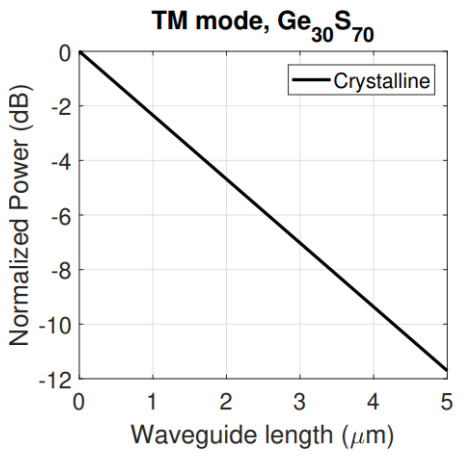

(c)

Figure 5.45: Crystalline $\mathrm{Ge}_{30} \mathrm{~S}_{70}$ patterned on the top surface of silicon waveguide in crystalline phase. a) Intensity profile of TM mode, b) Intensity distribution along the waveguide, and c) Output power of TM mode as a function of the length of the waveguide.

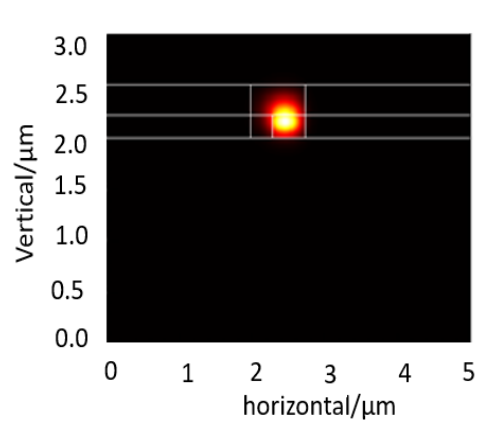

(a)

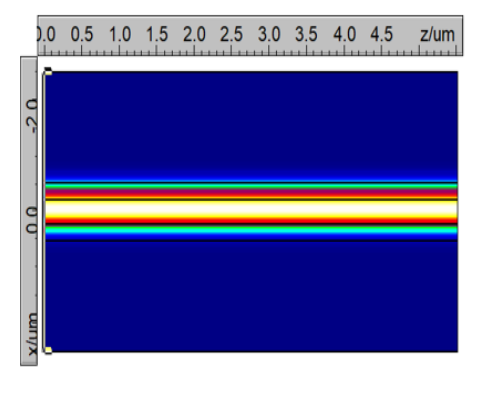

(b)

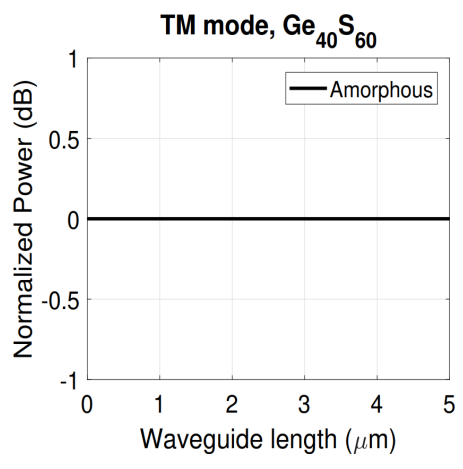

(c)

Figure 5.46: Amorphous $\mathrm{Ge}_{40} \mathrm{~S}_{60}$ patterned on the top surface of silicon waveguide in crystalline phase. a) Intensity profile of TM mode, b) Intensity distribution along the waveguide, and c) Output power of TM mode as a function of the length of the waveguide. 


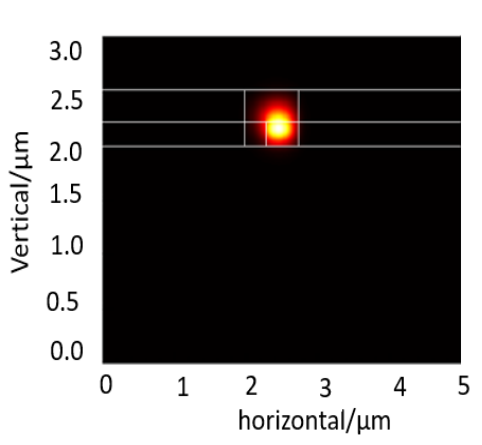

(a)

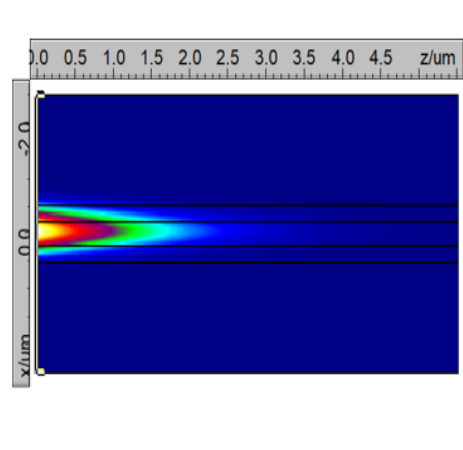

(b)

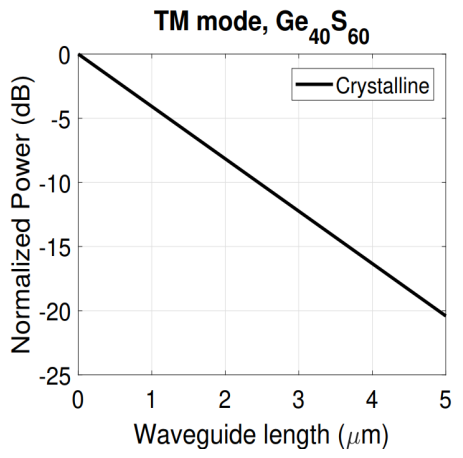

(c)

Figure 5.47: Crystalline $\mathrm{Ge}_{40} \mathrm{~S}_{60}$ patterned on the top surface of silicon waveguide in crystalline phase. a) Intensity profile of TM mode, b) Intensity distribution along the waveguide, and c) Output power of TM mode as a function of the length of the waveguide.

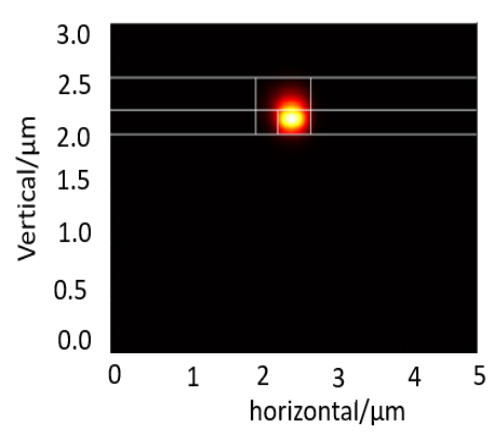

(a)

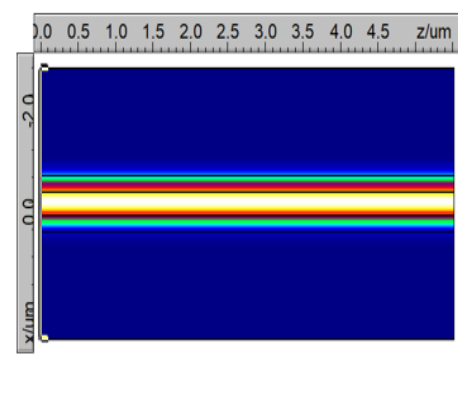

(b)

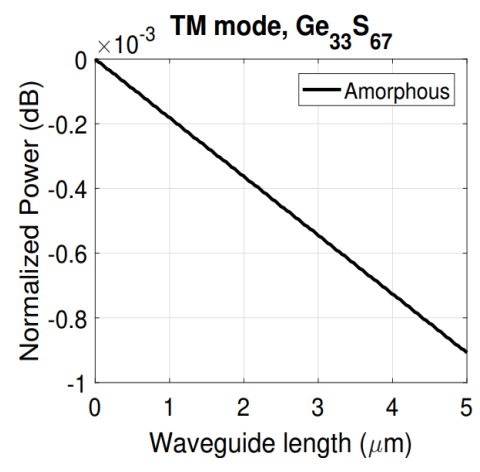

(c)

Figure 5.48: Amorphous $\mathrm{Ge}_{33} \mathrm{~S}_{67}$ patterned on the top surface of silicon waveguide in crystalline phase. a) Intensity profile of TM mode, b) Intensity distribution along the waveguide, and c) Output power of TM mode as a function of the length of the waveguide. 


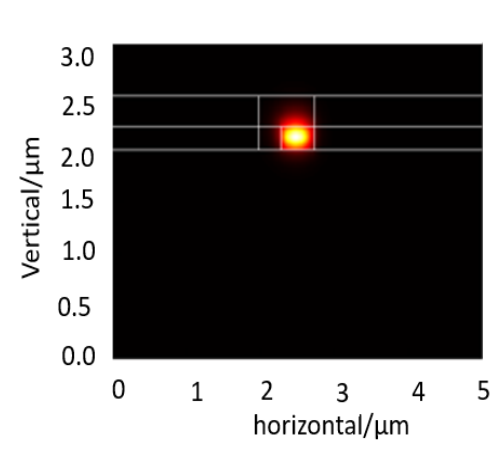

(a)

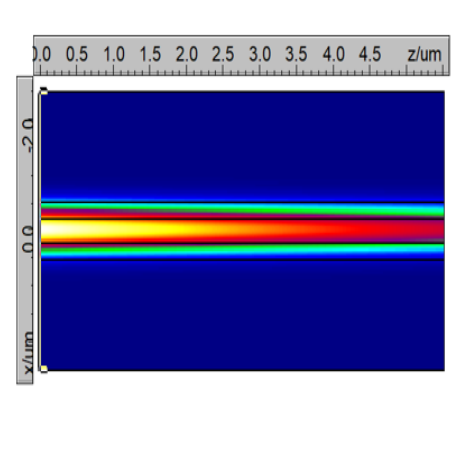

(b)

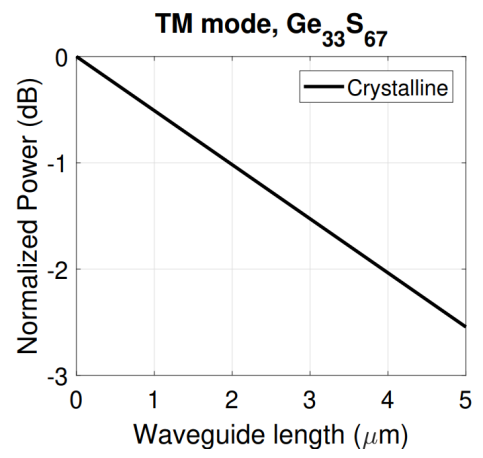

(c)

Figure 5.49: Crystalline $\mathrm{Ge}_{33} \mathrm{~S}_{67}$ patterned on the top surface of silicon waveguide in crystalline phase. a) Intensity profile of TM mode, b) Intensity distribution along the waveguide, and c) Output power of TM mode as a function of the length of the waveguide.

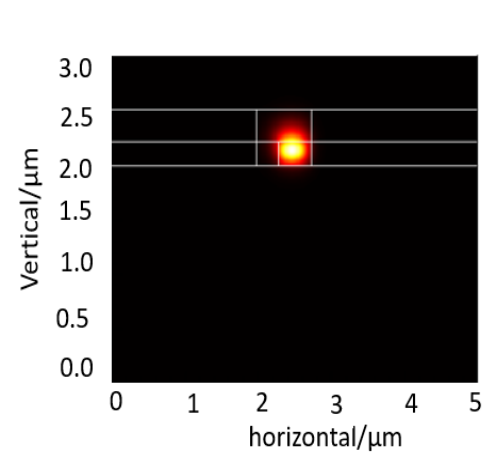

(a)

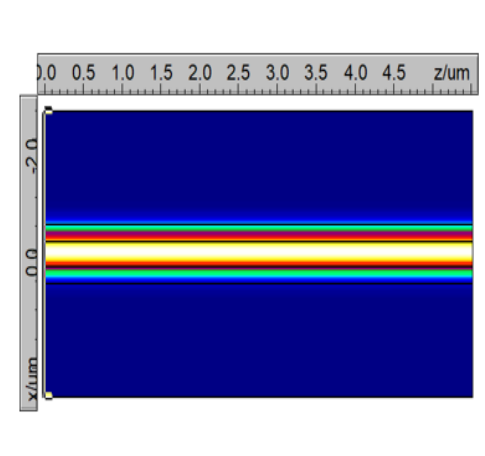

(b)

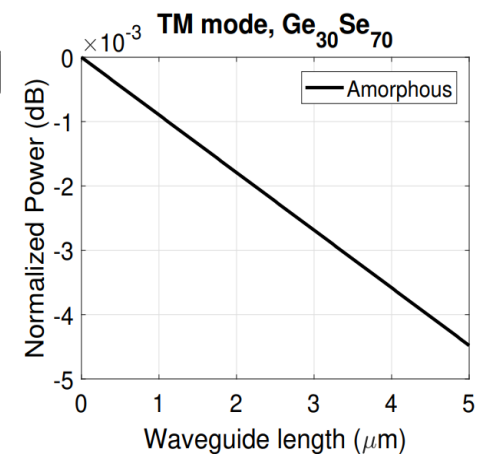

(c)

Figure 5.50: Amorphous $\mathrm{Ge}_{30} \mathrm{Se}_{70}$ patterned on the top surface of silicon waveguide in crystalline phase. a) Intensity profile of TM mode, b) Intensity distribution along the waveguide, and c) Output power of TM mode as a function of the length of the waveguide. 


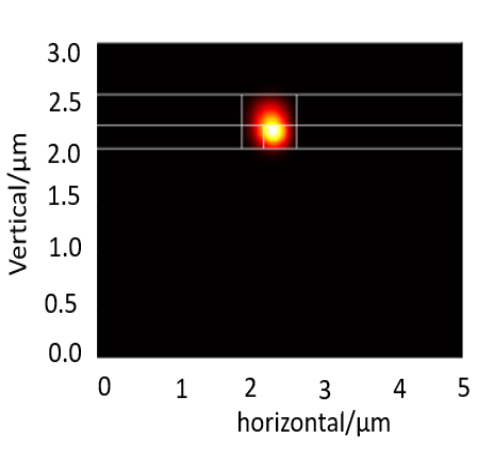

(a)

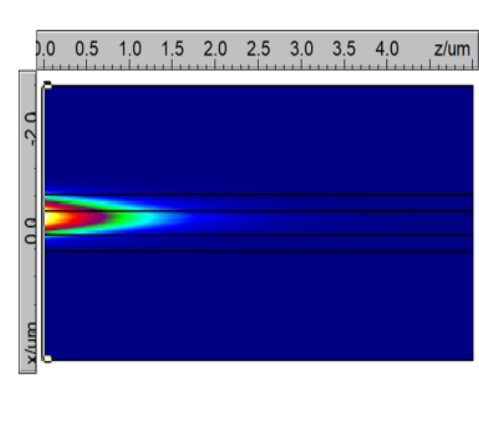

(b)

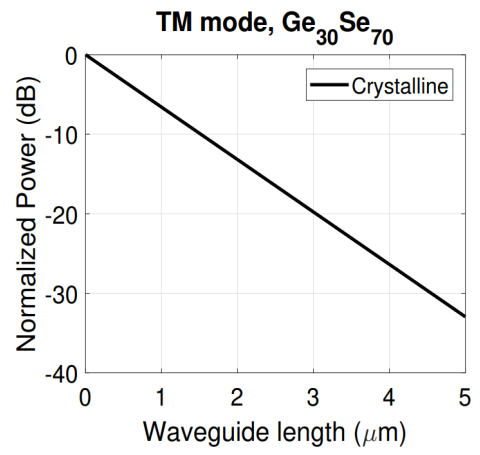

(c)

Figure 5.51: Crystalline $\mathrm{Ge}_{30} \mathrm{Se}_{70}$ patterned on the top surface of silicon waveguide in crystalline phase. a) Intensity profile of TM mode, b) Intensity distribution along the waveguide, and c) Output power of TM mode as a function of the length of the waveguide.

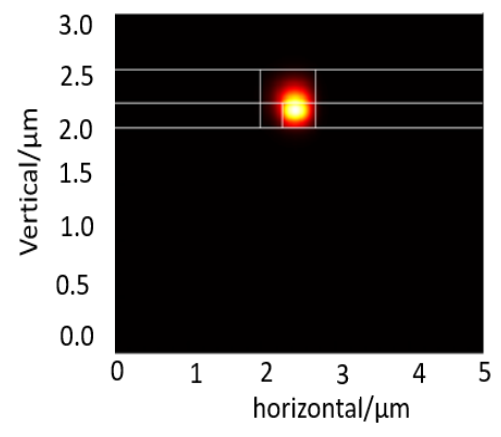

(a)

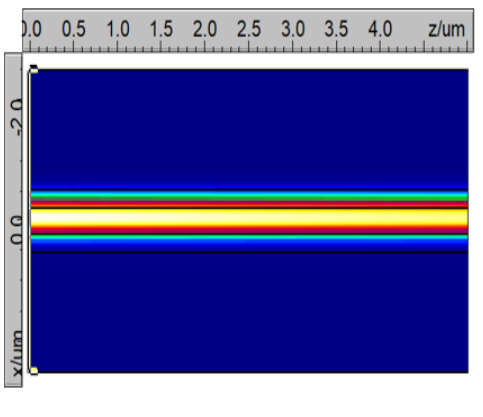

(b)

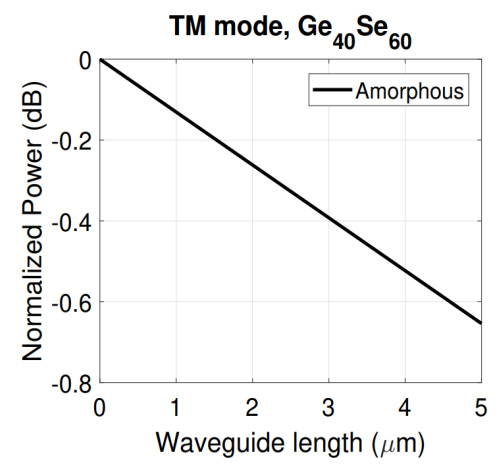

(c)

Figure 5.52: Amorphous $\mathrm{Ge}_{40} \mathrm{Se}_{60}$ patterned on the top surface of silicon waveguide in crystalline phase. a) Intensity profile of TM mode, b) Intensity distribution along the waveguide, and c) Output power of TM mode as a function of the length of the waveguide. 


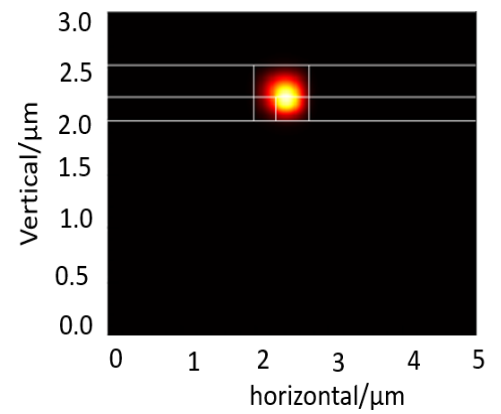

(a)

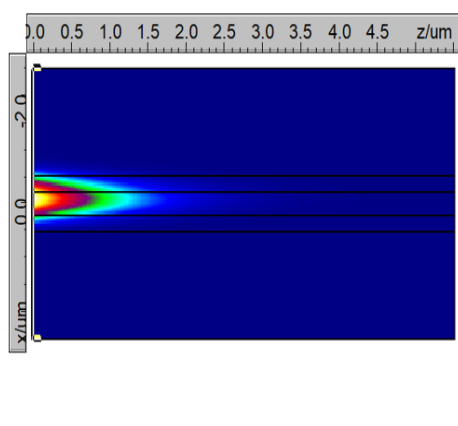

(b)

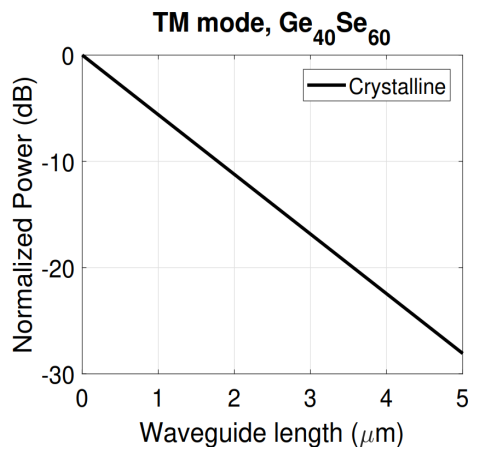

(c)

Figure 5.53: Crystalline $\mathrm{Ge}_{40} \mathrm{Se}_{60}$ patterned on the top surface of silicon waveguide in crystalline phase. a) Intensity profile of TM mode, b) Intensity distribution along the waveguide, and c) Output power of TM mode as a function of the length of the waveguide.

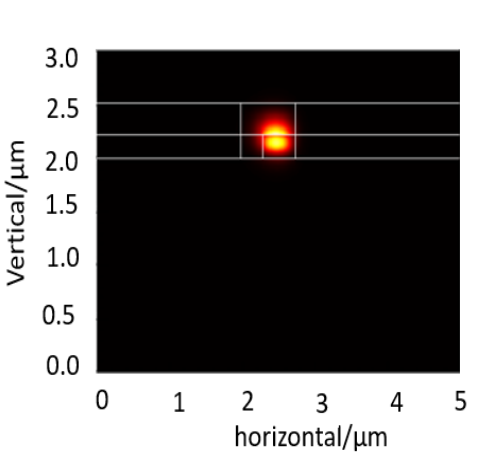

(a)

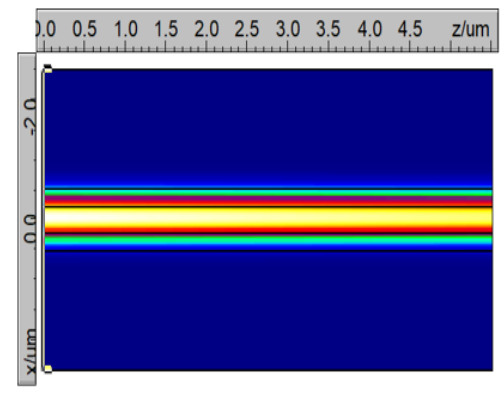

(b)

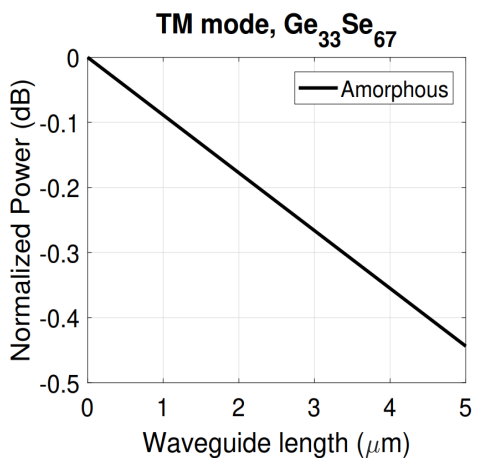

(c)

Figure 5.54: Amorphous $\mathrm{Ge}_{33} \mathrm{Se}_{67}$ patterned on the top surface of silicon waveguide in crystalline phase. a) Intensity profile of TM mode, b) Intensity distribution along the waveguide, and c) Output power of TM mode as a function of the length of the waveguide. 
Table 5.19: Fundamental TM mode loss in Waveguide design 4 with inhouse synthesized inks.

\begin{tabular}{cccc} 
Length & $\begin{array}{c}\text { Propagation loss } \\
\text { Amorphous } \\
(\mu \mathrm{m})\end{array}$ & $\begin{array}{c}\text { Propagation loss } \\
\text { Crystalline } \\
(\mathrm{dB} / \mu \mathrm{m})\end{array}$ \\
\hline $\mathrm{Ge}_{30} \mathrm{~S}_{70}$ & 3 & 0 & -2.3 \\
\hline $\mathrm{Ge}_{40} \mathrm{~S}_{60}$ & 3 & -0 & -4.09 \\
\hline $\mathrm{Ge}_{33} \mathrm{~S}_{67}$ & 3 & 0 & -0.51 \\
\hline $\mathrm{Ge}_{30} \mathrm{Se}_{70}$ & 3 & 0 & -6.52 \\
\hline $\mathrm{Ge}_{40} \mathrm{Se}_{60}$ & 3 & -0.11 & -5.63 \\
\hline $\mathrm{Ge}_{33} \mathrm{Se}_{67}$ & 3 & -0.08 & -0.44 \\
\hline
\end{tabular}

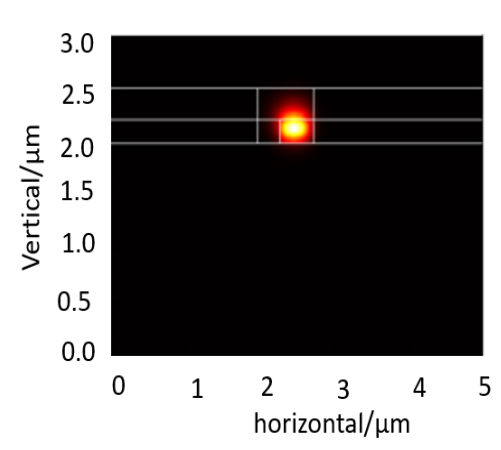

(a)

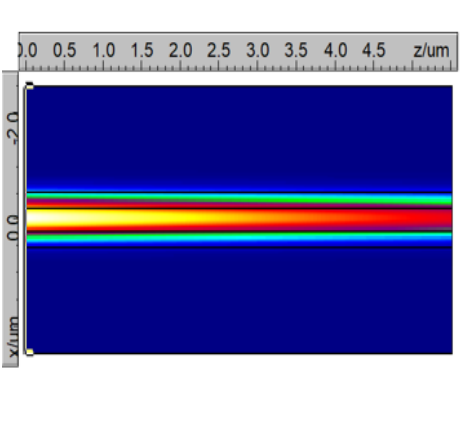

(b)

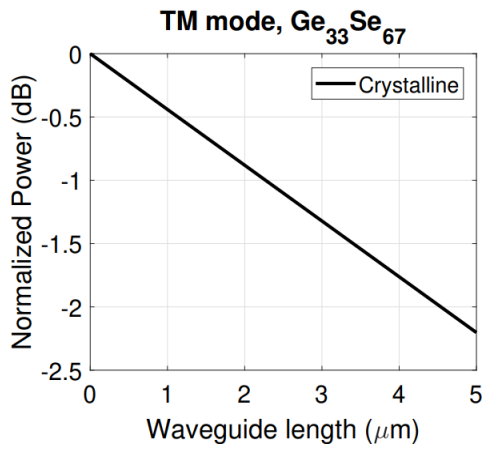

(c)

Figure 5.55: Crystalline $\mathrm{Ge}_{33} \mathrm{Se}_{67}$ patterned on the top surface of silicon waveguide in crystalline phase. a) Intensity profile of TM mode, b) Intensity distribution along the waveguide, and c) Output power of TM mode as a function of the length of the waveguide.

Comparison of all waveguide design losses in fundamental TM mode in amorphous and crystalline phases of six in-house synthesized inks are reported in Table 5.20. From the propagation loss calculations from simulations, it is observed that all compositions except $\left(\mathrm{Ge}_{33} \mathrm{Se}_{67}\right.$ and $\left.\mathrm{Ge}_{33} \mathrm{~S}_{67}\right)$ show a considerable propagation loss in crystalline phase. The reason is that $\mathrm{Ge}_{33} \mathrm{Se}_{67}$ and $\mathrm{Ge}_{33} \mathrm{~S}_{67}$ in both phases have small 
Table 5.20: Comparison of TM mode losses in all waveguide design with in-house synthesized inks.

\begin{tabular}{c|cc|cc|cc}
\hline \multirow{2}{*}{ Composition } & \multicolumn{2}{|c}{ Design 1 } & \multicolumn{2}{c|}{ Design 3 } & \multicolumn{2}{c|}{ Design 4 } \\
& $\begin{array}{c}\text { Amorphous } \\
(\mathrm{dB} / \mu \mathrm{m})\end{array}$ & $\begin{array}{c}\text { Crystalline } \\
(\mathrm{dB} / \mu \mathrm{m})\end{array}$ & $\begin{array}{c}\text { Amorphous } \\
(\mathrm{dB} / \mu \mathrm{m})\end{array}$ & $\begin{array}{c}\text { Crystalline } \\
(\mathrm{dB} / \mu \mathrm{m})\end{array}$ & $\begin{array}{c}\text { Amorphous } \\
(\mathrm{dB} / \mu \mathrm{m})\end{array}$ & $\begin{array}{c}\text { Crystalline } \\
(\mathrm{dB} / \mu \mathrm{m})\end{array}$ \\
\hline $\mathrm{Ge}_{30} \mathrm{~S}_{70}$ & 0 & -2.42 & 0 & -2.05 & 0 & -2.34 \\
$\mathrm{Ge}_{40} \mathrm{~S}_{60}$ & 0 & -3.95 & 0 & -3.66 & 0 & -4.09 \\
$\mathrm{Ge}_{33} \mathrm{~S}_{67}$ & -0.08 & -0.44 & 0 & -0.45 & 0 & -0.51 \\
$\mathrm{Ge}_{30} \mathrm{Se}_{70}$ & 0 & -6.08 & 0 & -5.64 & 0 & -6.52 \\
$\mathrm{Ge}_{40} \mathrm{Se}_{60}$ & -0.12 & -5.19 & -0.11 & -4.81 & -0.13 & -5.63 \\
$\mathrm{Ge}_{33} \mathrm{Se}_{67}$ & -0.08 & -0.440 & -0.08 & -0.4 & -0.08 & -0.44 \\
\hline
\end{tabular}

changes in the material structure and band gap during the crystallization process which are homogeneous in their nature [93]. $\mathrm{Ge}_{30} \mathrm{Se}_{70}$ shows the highest propagation loss difference between amorphous and crystalline phases. In Table 3.1 the extinction coefficient of $\mathrm{Ge}_{30} \mathrm{Se}_{70}$ in crystalline phase is bigger than extinction coefficient in amorphous phase which indicated higher loss in crystalline phase compare to amorphous phase. We expect to observe abrupt change in transmitted output power by increasing the temperature beyond the crystalline temperature in $\mathrm{Si}$ :ChG waveguide based temperature sensor with covering by this composition.

\subsection{Summary}

In this chapter, the Silicon waveguide: $\mathrm{ChG}$ based temperature sensor is simulated. The optical properties of each of synthesized Ge-S and Ge-Se ChGs is used in the modeling. The simulation results shows light confinement in Si core in amorphous phase of the ChG. In the crystalline phase of $\mathrm{ChG}$, the waveguide generates SPP modes at the interface between silicon and $\mathrm{ChG}$, confining light at the interface of $\mathrm{Si}$ and ChG. Abrupt changes is transmitted power occur in the crystalline phase. This forms the basis of the working principle of the integrated waveguide based temperature 
sensor. The next chapter goes over the fabrication and characterization of waveguide based temperature sensor. 


\section{CHAPTER 6:}

\section{FABRICATION OF SILICON:CHG WAVEGUIDE BASED PLASMONIC TEMPERATURE SENSORS}

Here, we discuss the fabrication of integrated waveguide based temperature sensors utilizing silicon waveguide and chalcogenide glasses. The process work described herein was performed at the Idaho Microfabrication Laboratory (IML) and Lurie Nanofabrication Facility (LNF) at University of Michigan.

\subsection{Fabrication Procedure}

Before we get to the technical details, the first section is a brief introduction of the entire process flow. The process flow for fabricating hybrid silicon:ChG waveguide based plasmonic temperature sensors is shown in Figure. 6.1. The process starts with a SOI substrate with device layer $0.22 \mu m$ and an oxide layer of $3 \mu m$. After cleaning the substrate, we apply the e-beam resist using a spin coater. Electron-Beam Lithography (EBL) is used to define the waveguide structure on the e-beam resist. Next, the defined pattern is transferred to the top Si layer by reactive ion etching (RIE). Finally, the remaining resist is removed using stripper and oxygen plasma. The waveguide section is covered by $\mathrm{ChG}$ using thermal evaporation and ink printing methods. Finally, an $\mathrm{SiO}_{2}$ layer is deposited on the wafer as the upper-cladding. The details of the wafer preparation are provided in appendix B. 


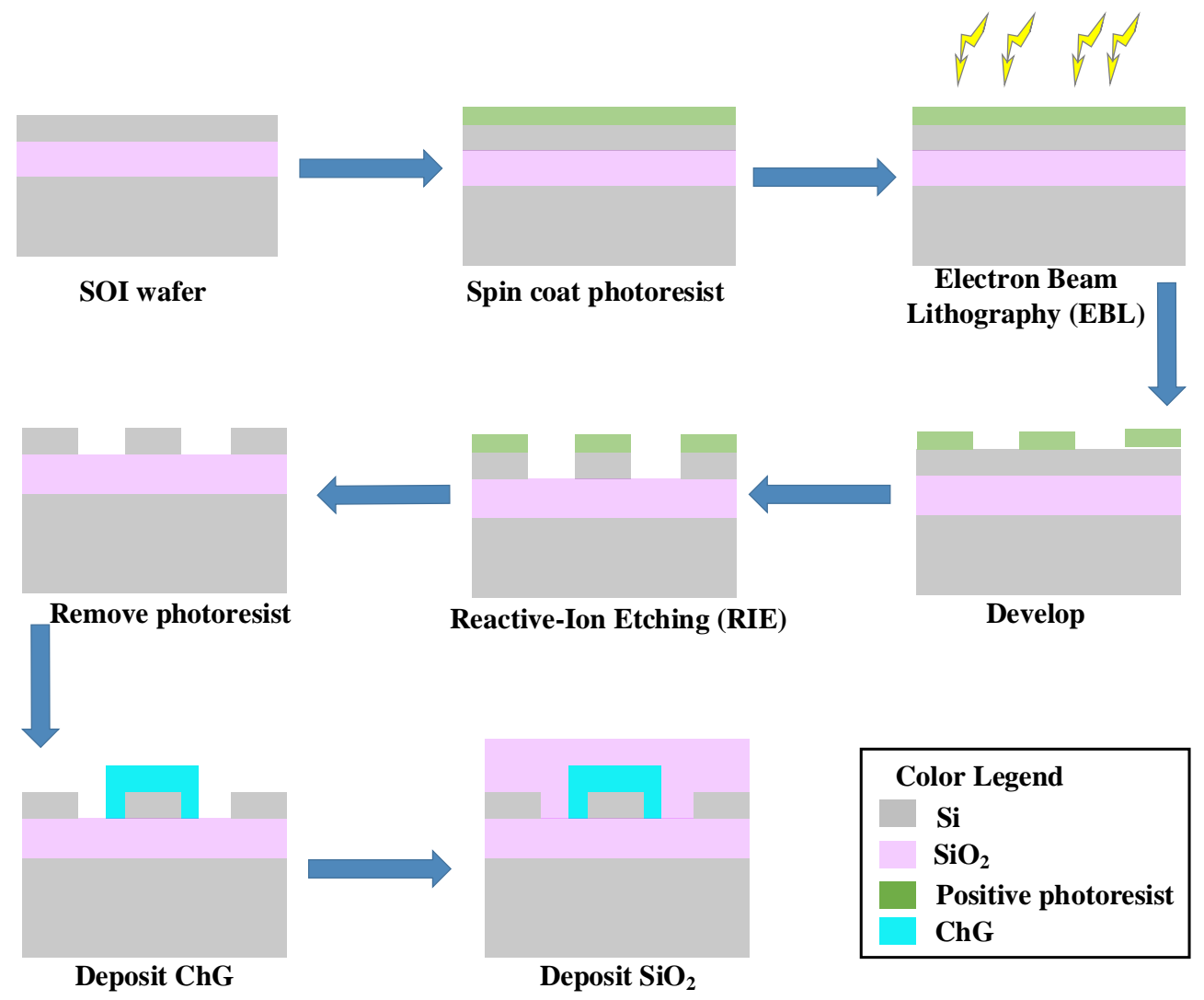

Figure 6.1: Process flow for fabricating Silicon:ChG waveguide based plasmonic temperature sensors

For the fabrication of silicon waveguides terminated with grating couplers, we used EBL. This method is one of the key fabrication techniques that allows us to create patterns at the nanoscale level and test prototypes in the lab. EBL is a direct-write method and does not need a prefabricated mask. The EBL working principle is relatively simple and very similar to photolithography. In this method, a focused beam of electrons is scanned across a substrate covered by an electronsensitive resin (or e-beam resist). According to electron beam energy the photoresist solubility properties change. This process provides two significant benefits over 
conventional photolithography: (i) smaller features are possible due to the narrow width of the electron beam writing the pattern and (ii) greater freedom to evaluate new device designs as it only requires patterns created by CAD without the need for creating photomasks. However, EBL is a slower process compared to conventional photolithography as the exposure window is much smaller $(\sim 600 \mu m \times \sim 600 \mu m)$ and can only write on one sample at a time. After the wafer is patterned with the instructions coming from the pattern generator, areas exposed, or not exposed according to the tone of the resin, are removed by developing the exposed e-beam resist pattern. The column and chamber are in high vacuum. The column in contains all elements to create, accelerate, focus/defocus, and turn the beam on/off. Chamber contains a stage to place the sample. The dimension of the chamber determines the size and accessibility of the sample that can be patterned. A computer-controlled high-accuracy five-axis stage is used precisely move and rotate and tilt the stage. In addition, the chamber is equipped with a charged coupled device (CCD) camera to visualize inside the chamber and assess the control of the sample positioning. The chamber is isolated from mechanical vibration, which is very critical for high resolution EBL. Since the discovery of polymethylmethacrylate (PMMA) as an electron resist by Hatzakis [121] in 1969, EBL has been used to fabricate a wide variety devices, ranging from integrated circuits, [122,123], to photonic crystals, [124-127] experiments. The following figure will describe the steps used to fabricate this waveguide device.

In the proposed waveguide based temperature sensor, two design of waveguides: a) Waveguide grating coupler, and b) S-bend waveguides are considered to confirm the functionality of the device. First, we introduce the waveguide grating coupler fabrication and testing results, then the S-bend device will be discussed. 


\subsection{Waveguide Grating Coupler Fabrication}

The selection of proper writing dose in EBL is important to correct the point exposure time. Several parameters such as beam energy, substrate compositions, pattern line width and pattern density have effect on selection of the dose. We started the fabrication by studying the effect of dose on each section of the design.

\subsubsection{Dose test on grating coupler section}

Due to the different feature sizes in the proposed design structure, the design was divided into three different sections: 1) grating coupler, 2) taper, and 3) waveguide. The schematic of the desired structure on SOI wafer is shown in Figure. 6.2.

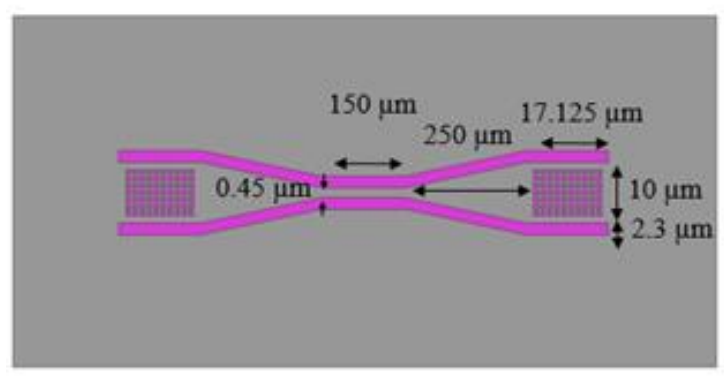

(a)

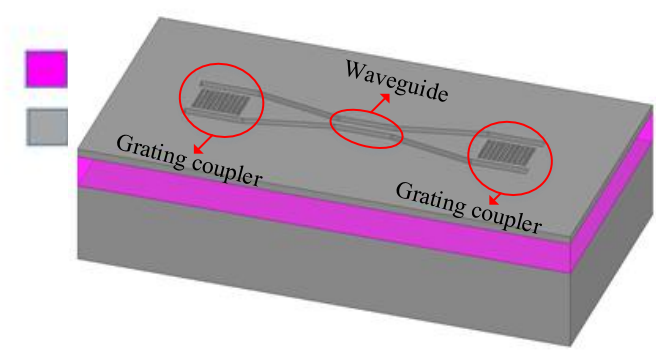

(b)

Figure 6.2: Proposed waveguide grating coupler design, a) Top view, b) 3D view. A waveguide is connected to input/output grating coupler using two taper waveguides. Waveguide width $=0.45 \mu \mathrm{m}$, waveguide length $=150 \mu \mathrm{m}$, taper length $=250 \mu \mathrm{m}$, grating coupler length $=17.125 \mu \mathrm{m}$, and grating coupler width $=14.6 \mu \mathrm{m}$.

The schematic of the grating coupler is shown in Figure. 6.3. Table 6.1 shows the designed parameters of grating couplers. 
Table 6.1: Sweep grating coupler parameters

\begin{tabular}{ccccc}
\hline Design & $\mathrm{P}_{H}(\mu m)$ & $\mathrm{w}(\mu m)$ & $\mathrm{P}_{v}(\mu m)$ & $\mathrm{L}(\mu m)$ \\
\hline $\mathrm{D} 1$ & 0.388 & 0.115 & 0.685 & 0.39 \\
\hline $\mathrm{D} 2$ & 0.388 & 0.120 & 0.685 & 0.39 \\
\hline $\mathrm{D} 3$ & 0.388 & 0.122 & 0.685 & 0.39 \\
\hline $\mathrm{D} 4$ & 0.388 & 0.124 & 0.685 & 0.39 \\
\hline $\mathrm{D} 5$ & 0.388 & 0.125 & 0.685 & 0.39 \\
\hline $\mathrm{D} 6$ & 0.388 & 0.130 & 0.685 & 0.39 \\
\hline
\end{tabular}

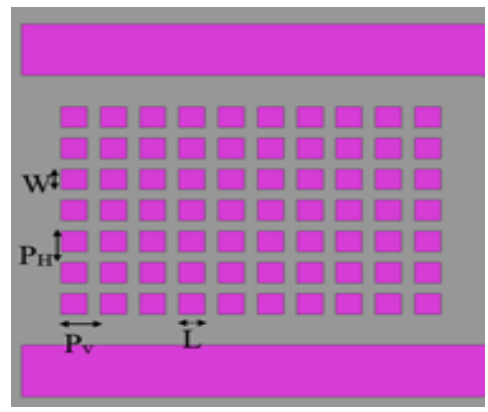

(a)

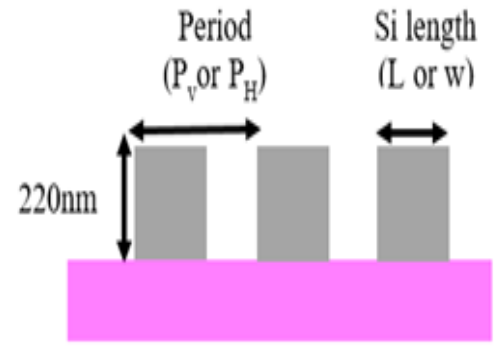

(b)

Figure 6.3: Grating coupler, a) top view, b) side view. $\mathbf{P}_{v}$ is vertical grating period, $\mathbf{P}_{h}$ is horizontal grating period, $\mathbf{L}$ is length of grating pitch, and $\mathrm{W}$ is width of grating pitch. Device layer (Si) thickness is $220 \mathrm{~nm}$.

For each section, different dose tests were studied during EBL patterning to figure out optimum dose which could allow for complete penetration of the electron beams into the entire thickness of the PMMA resist. Fully etched grating coupler (schematic in Figure. 6.4, taper lines, and waveguides were patterned using appropriate calibrated dose rates and energy. 


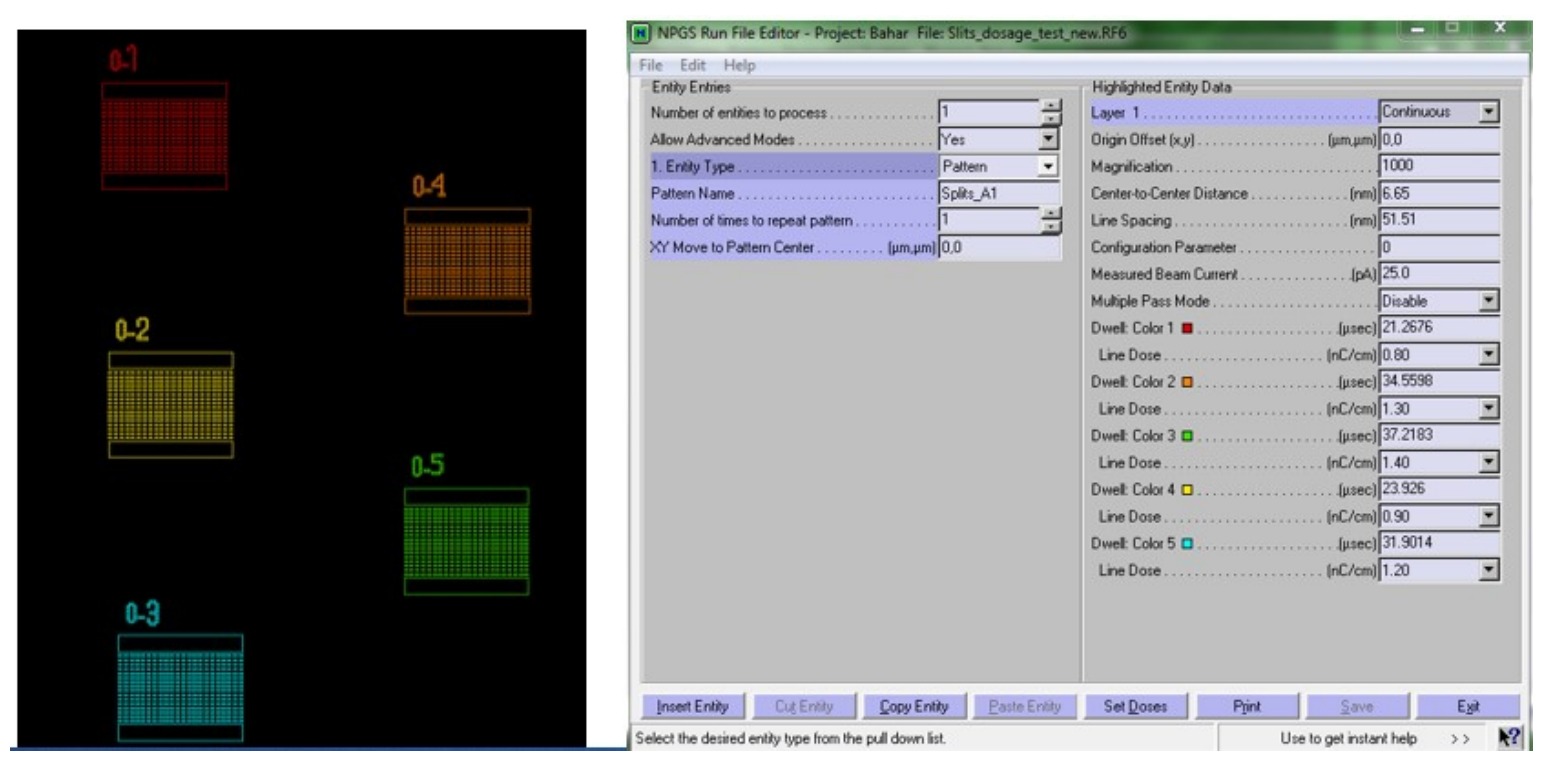

Figure 6.4: Grating couple design in DesignCAD with different dose. Each color shows exposure beam dose in Run File Editor in NPGs software.

First, we used low line dose $(0.9,1.20,1.40 \mathrm{nC} / \mathrm{cm})$ with $25 \mathrm{pA}$ beam current and $30 \mathrm{keV}$ energy to pattern the grating coupler. Figure. 6.4 shows the design used to conduct this part of the test. In DesignCAD software, each color indicates a different dose. Once the pattern was designed in DesignCAD, the Run Filed Editor in Nanometer Pattern Generation System (NPGS) software was used to record the exposure conditions for the different drawing elements in the pattern. Also, Magscale parameter in DesignCAD showed ideal magnification depending on writing field and microscope which can be used in Run File Editor.

After patterning, the samples were imaged using AFM to check the effect of each line dose value on the pattern quality. The measurement results are illustrated in Figure. 6.5. In these samples, the PMMA thickness was $\sim 250 \mathrm{~nm}$. According to line dose setting in NPGS software shown in Figure. 6.4, grating couplers numbered 0-1 was patterned with line dose of $0.9 \mathrm{nC} / \mathrm{cm}$. The ripples in the curves show pitches 
in grating coupler depth inside the PMMA. The depth of these ripples should be the same as PMMA thickness. 2D AFM results shows that the beam could not penetrate through the PMMA thickness.

Also, the 3D image shown in Figure. 6.4 confirms that the grating coupler was underexposed. Then, we increased the line dose to 1.2 , and $1.4 \mathrm{nC} / \mathrm{cm}$ to pattern the grating coupler which are shown in Figure. 6.5(b,c) respectively. In these samples, the PMMA thickness was $\sim 250 \mathrm{~nm}$. The ripples in the curves show pitches in grating coupler depth inside the PMMA. By comparing the results in Figure. 6.5, it can be seen that a low dose cannot penetrate through the entire thickness of the photoresist and a higher line dose is required to pattern this section of the device. Then, we studied high line doses for a grating coupler section. A higher beam energy causes deeper penetration into photoresist thickness and consequently fewer secondary electrons reach the surface. So, to pattern the grating coupler line dose of $1.5 \mathrm{nC} / \mathrm{cm}$ is selected. The AFM measurement curve and 3D images are shown in Figure. 6.6. It can be seen that the depth of pitches after developing the sample is similar to PMMA thickness. These results indicate that in order to pattern smaller dimension features, a high line dose is required. Thus, a high line dose of $1.5 \mathrm{nC} / \mathrm{cm}$ was selected for patterning grating couplers. The same procedure is followed for the taper part of the design. Among different line doses, $1.3 \mathrm{nC} / \mathrm{cm}$ provided the best results. Thus, this line dose was selected for patterning taper section. AFM images in Figure. 6.7 show that beam with $1.3 \mathrm{~nm} / \mathrm{cm}$ can reach the thickness of photoresist. In the waveguide section, as shown in Figure. 6.8, a line dose of $0.9 \mathrm{nC} / \mathrm{cm}$ was selected as a proper dose. 

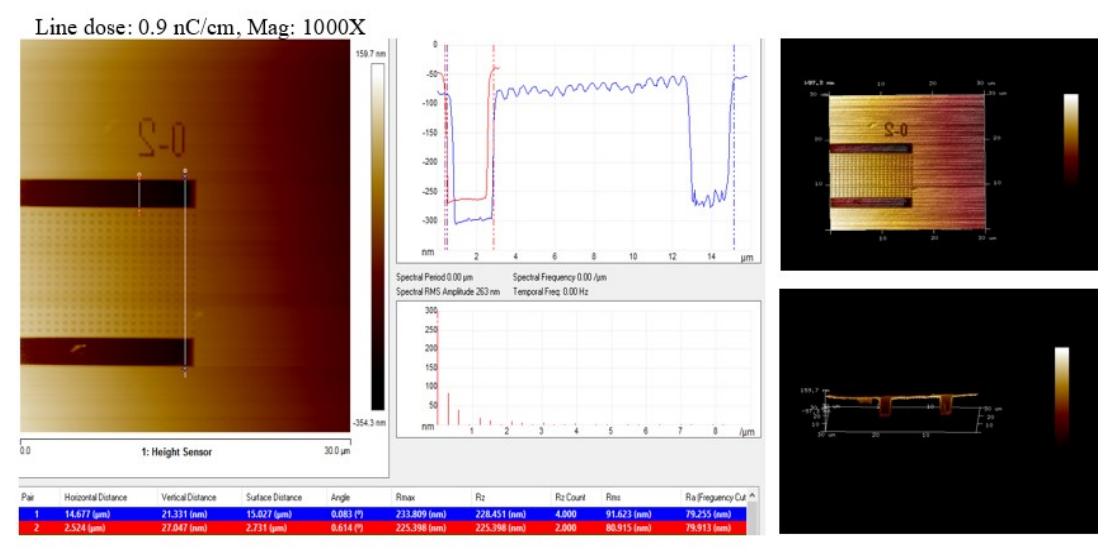

(a)

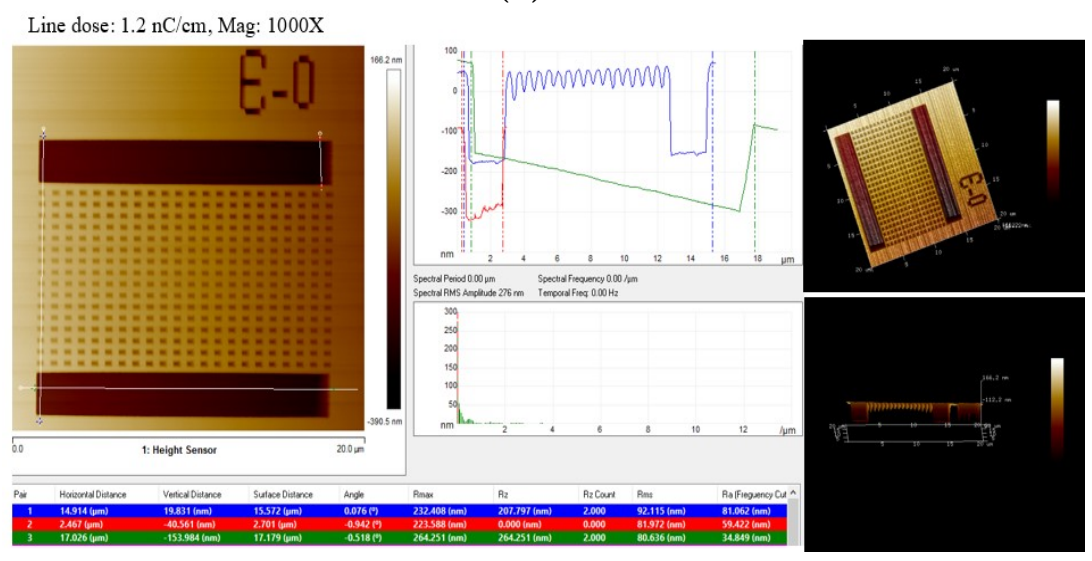

(b)

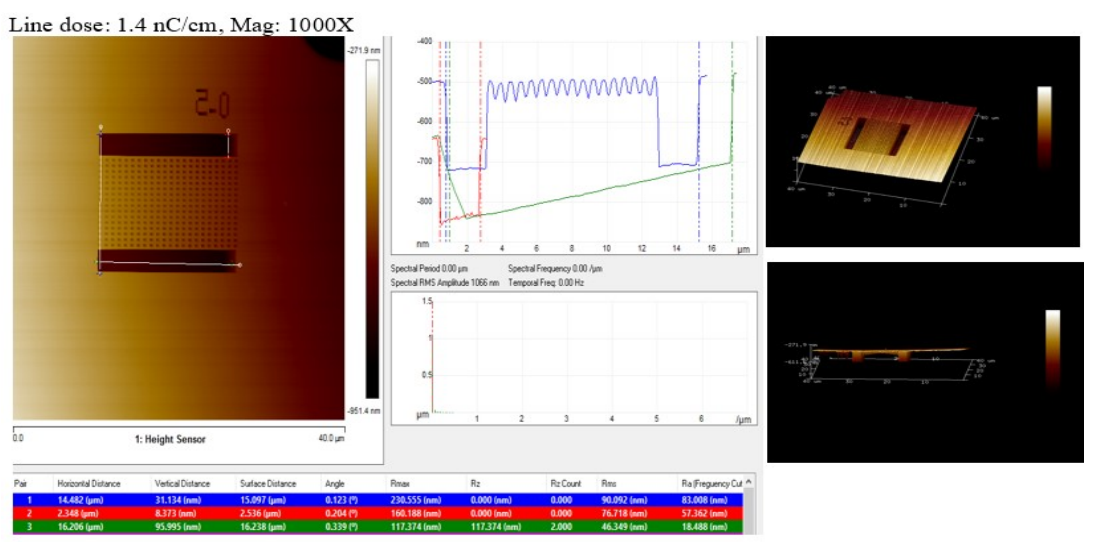

(c)

Figure 6.5: Low line dose effect test for grating coupler section of the design , a) $0.9 \mathrm{nC} / \mathrm{cm}$, b) $1.2 \mathrm{nC} / \mathrm{cm}$, c) $1.4 \mathrm{nC} / \mathrm{cm}$ 


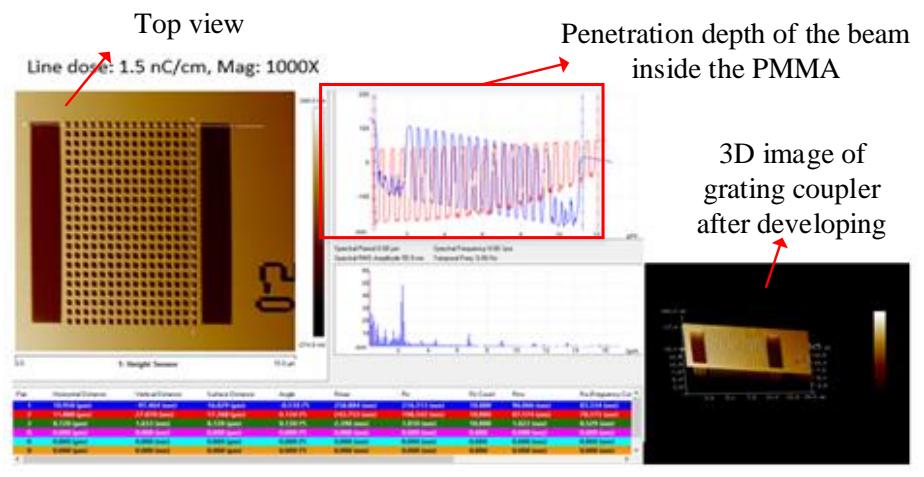

Figure 6.6: High line dose effect test for grating coupler section of the design. Dark areas are exposed by EBL.
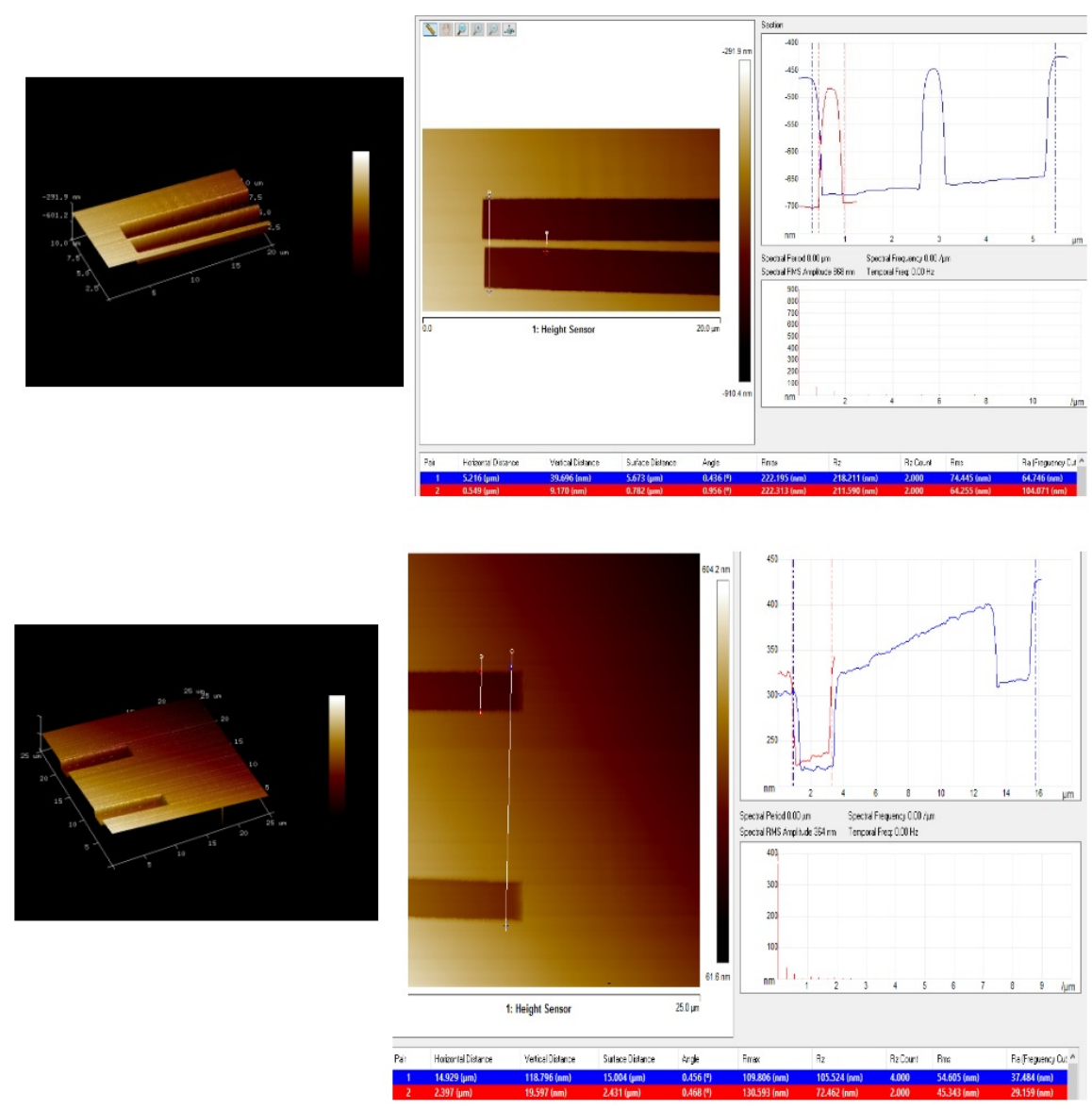

Figure 6.7: Line dose study on taper part of the design. 

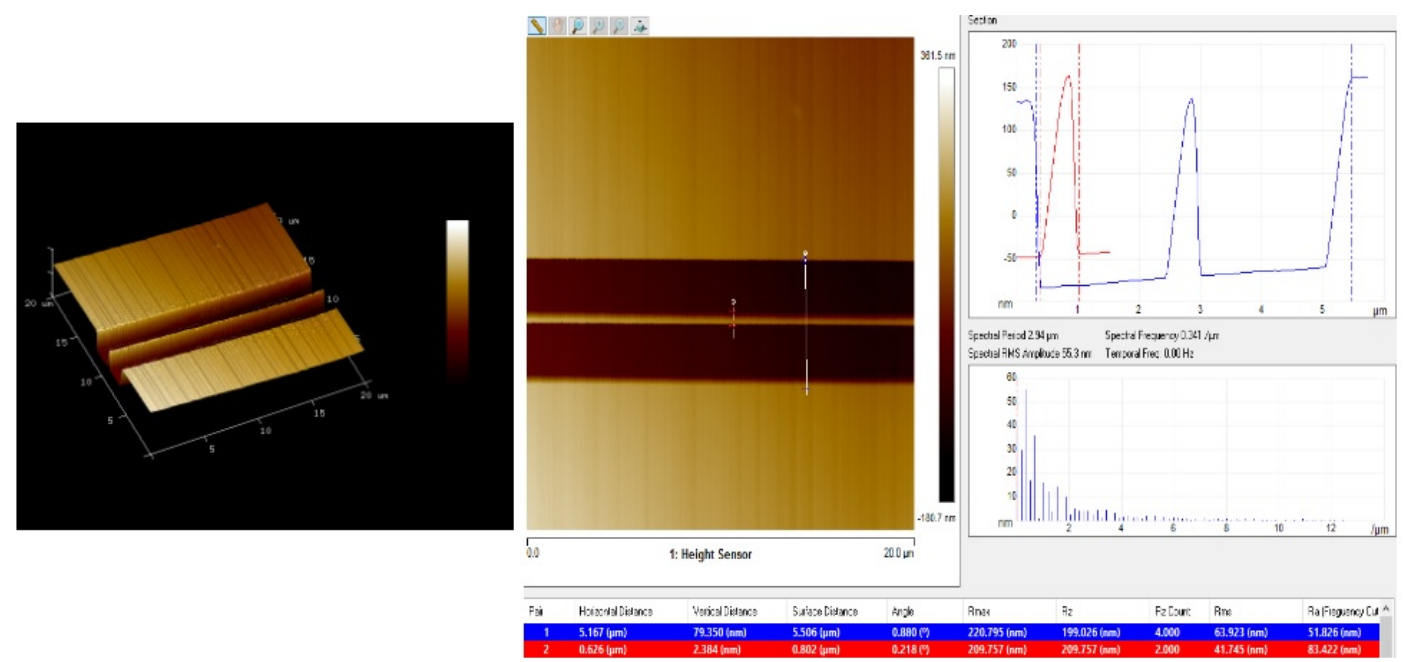

Figure 6.8: Line dose study on waveguide part of the design.

\subsubsection{Stitching}

Once we determined the optimum line dosages for the different parts of the design, we proceeded with the fabrication of the device structure. In modern EBL systems, large-area patterns are formed by stitching together a mosaic of small fields or stripes. The EBL system has a limited area of exposure (writing field). Then, the large pattern is divided into multiple exposure fields side by side with stage movement between each field exposure so that the edge of each field accurately overlaps that of its nearest neighbor. The deviation from perfect alignment between writing fields is called a stitching error. This error causes small unintended discontinuities between the borders between adjacent fields. The performance of the photonic circuits can be affected by stitching errors due to an increase in the loss from radiative mode and back reflection of guided modes. Within NPGS, good stitching is a result of the correct combination of pattern writing size and accurate stage motion or accurate alignment. To produce writing fields that stitch together, the placement of each pattern must 
accurately correspond to the size of the pattern. In addition, the writing axes must be aligned to the stage axes to achieve good stitching.

\subsubsection{Blind stitching}

We first used a recommended fracture method to pattern the entire design aslarge writing areas, wherein each writing field being stitched together must have unique pattern features, NPGS fracture mode was used. NPGS can write a single CAD file that covers a very large area by breaking the large file into smaller pieces, wherein each piece defines a sub-field for writing. When this mode is used, NPGS will determine the correct stage coordinates so that the sample will be moved and the pieces will be written at the correct position, within the limitation of stage accuracy. The precision of the stage in our system is 4 digits. The written files are all offset so that each file is appropriately centered in the writing field that will be offset by the stage movement. The viewable files are not offset, so that when they are loaded into DesignCAD, they will match up to recreate the original unfractured pattern. First, we use blind stitching to pattern whole structure. In this method, stitching the sub-field are done without dedicating alignment marks at each field and blindly addressing the EBL exposure. The outcome depends on the accuracy of the stage and needs better calibration for the writing field.

To find the stage error in $x$ and $y$ direction, we fractured the entire design into $80 \mu \mathrm{m}$ sub-fields with $20 \mu \mathrm{m}$ borders. According to sub-field size and border, the magnification is calculated by NPGS, which is about 1000X. Based on our dose test, we selected a line dose of $1.7 \mathrm{nC} / \mathrm{cm}$ for grating, $1.3 \mathrm{nC} / \mathrm{cm}$ for taper and $0.9 \mathrm{nC} / \mathrm{cm}$ for waveguide sections. The relative stage movement with 4 digits precision is about 
$x= \pm 0.079 \mathrm{~mm}$ and $y= \pm 0.0003 \mathrm{~mm}$. An optical microscope image of patterned design is shown in Figure. 6.9.
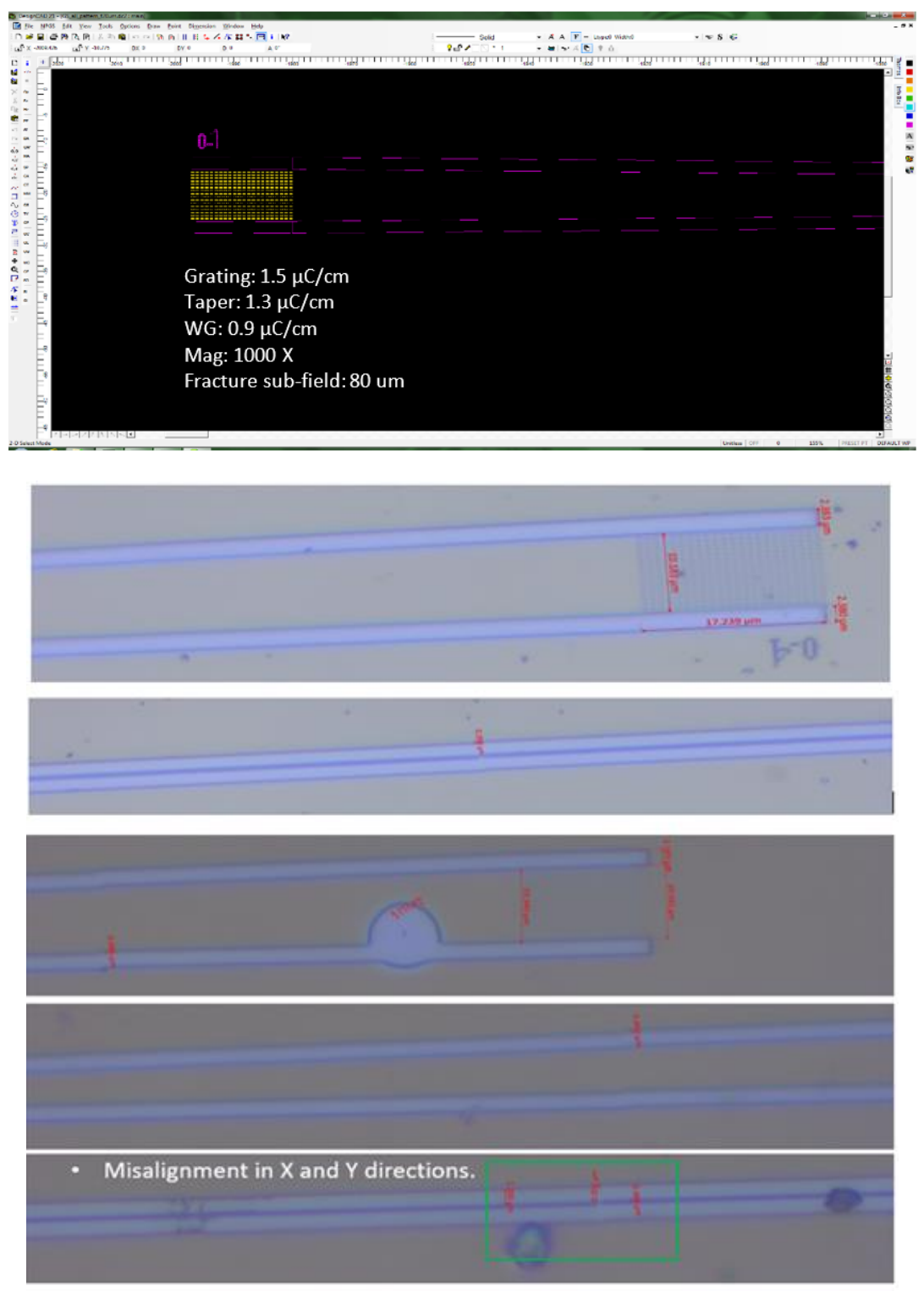

Figure 6.9: Fracture with $80 \mu \mathrm{m}$ sub-fields.

To decrease the misalignment in $x$ and $y$ directions, we aligned the chip's $x$ axis 
to the stage $x$ axis. After patterning, $x$ offset value was measured with microscope and this value was subtracted from sub-field value which indicates the relative $x$ stage movement. Also, for $y$ misalignment, a global stage correction is applied. To determine this, the sample was carbon coated and imaged using scanning electron microscope (SEM). The value of the gap between two subfields was measured. Then, by applying these values, NPGS can calculate the stage rotation offset during movement. Also, if there is still some misalignment in $y$ direction, the value can be measured with microscope and subtracted from relative $y$ stage movement. We fractured the structure with the same subfield sizes of $80 \mu m$ with $20 \mu m$ border and with 1000X magnification. Based on the misalignment values in $x$ and $y$ directions from prior sample, the relative $x$ stage movement was selected as $0.0788 \mathrm{~mm}$ and $y$ relative movement was selected as $0.0003 \mathrm{~mm}$. The images of the patterned samples are shown in Figure. 6.10.

As shown in Figure. 6.10, in stitching taper line with waveguide, proximity effect occurs which is due to using different dose in each sub-field. The gap in waveguide section is very small. Changing dose in this section causes a missing waveguide. 

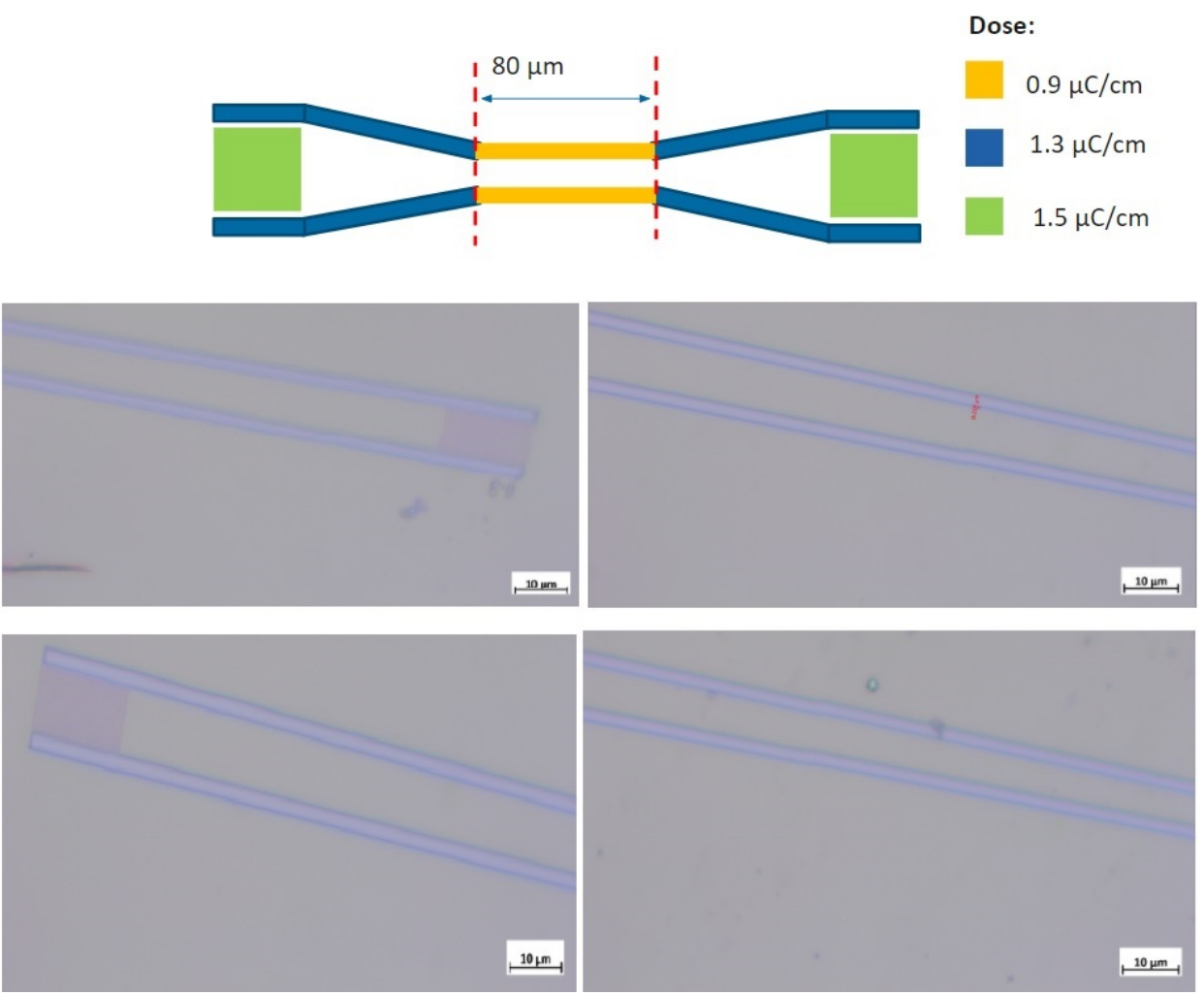

Figure 6.10: Fracture after fixing stage, sub-field $=80 \mu \mathrm{m}$.

We decided to increase the sub-field to $95 \mu \mathrm{m}$ with $5 \mu \mathrm{m}$ border, which covers an entire waveguide and a portion of the taper. In this case, waveguide can be patterned in one sub-field which reduces the emergence of misalignment in this part. In this case, Mag is equal to $1000 \mathrm{X}, x$ relative movement is $0.0938 \mathrm{~mm}$ and $y$ relative movement is $0.0003 \mathrm{~mm}$. The image of waveguide section is shown in Figure. 6.11. As can be seen in this figure, we could not fix the misalignment in $y$ direction due to stage precision limitations. Values that provided best results were selected. 

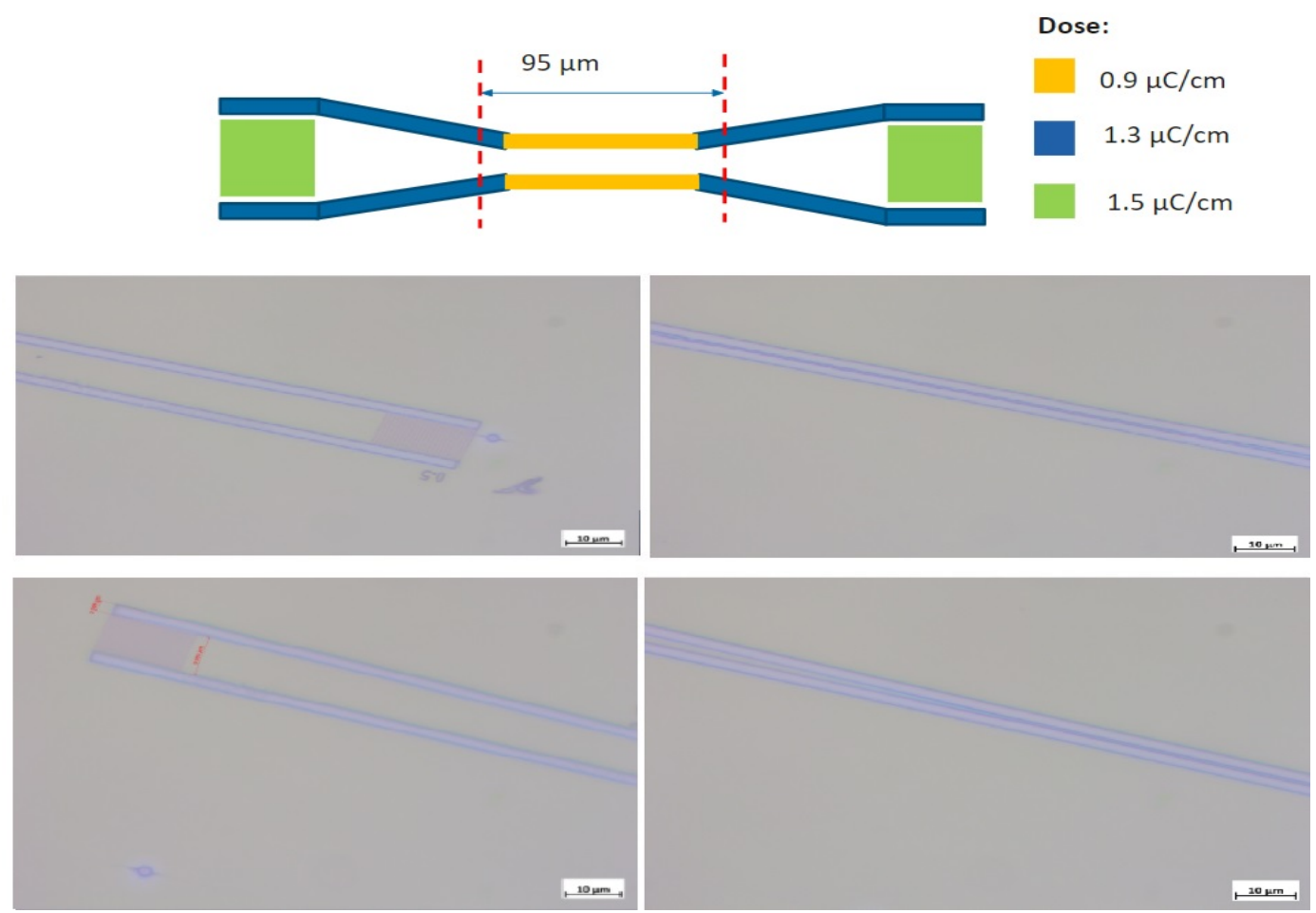

Figure 6.11: Fracture with sub-field $=95 \mu \mathrm{m}$.

The issue regarding blind stitching is that the writing axes must be carefully aligned to the stage movement. In order to pattern a device using blind stitching, the microscope should be equipped with an accurate stage. Also the writing field needs to be accurately calibrated to the both correct size and the orientation of the stage axes. The drift in the stage during the movement does not show consistent misalignment offset values and causes stitching errors as shown in Figure. 6.12. So, using this method to fabricate the device is not repeatable as the relative offset is manually entered to the yield the desire spacing in order to stitch the pattern together. Also, edges of the pattern mismatches as large as several microns due to irregularities in the stage movement. Since the stage on the microscope in our EBL tool is not accurate 
enough to produce good stitching results, using registration marks in each writing field will be helpful.
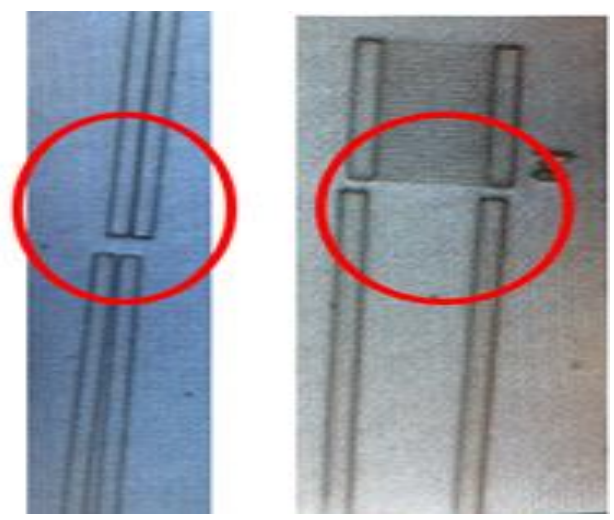

(a)

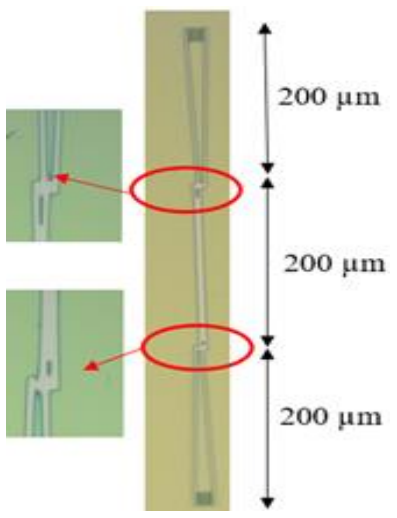

(b)

Figure 6.12: Blind stitching error, a) discontinuous, b) misalignment

\subsubsection{Stitching using registration marks}

As, we mentioned before to achieve high resolution grating coupler in the fabrication, the writing field should be divided into subfields. In order to avoid the stitching error, using the alignment corrections to stitch the subfields properly is suggested. To this end, we decided to pattern registration marks on the SOI wafer prior to patterning waveguide structure. The NPGS alignment features can be used to align the marks using the exact conditions for each write. The alignment correction is to stitch the subfields properly. In order to have registration markers on the wafer, we have designed a photomask with marker's designs on it. The details of this photomask is discussed in the following section. 
6.2.2.2.1 Photo-mask fabrication The photomask pattern is generated using computer aided design (CAD) software and then sent to specialized manufacturers who transfer the pattern to a photomask. In each writing field, four registration marks are used to compensate the slow beam or stage drift. Each writing field should be aligned before it is written. The AutoCAD design of the photo-mask is shown in Figure. 6.13.

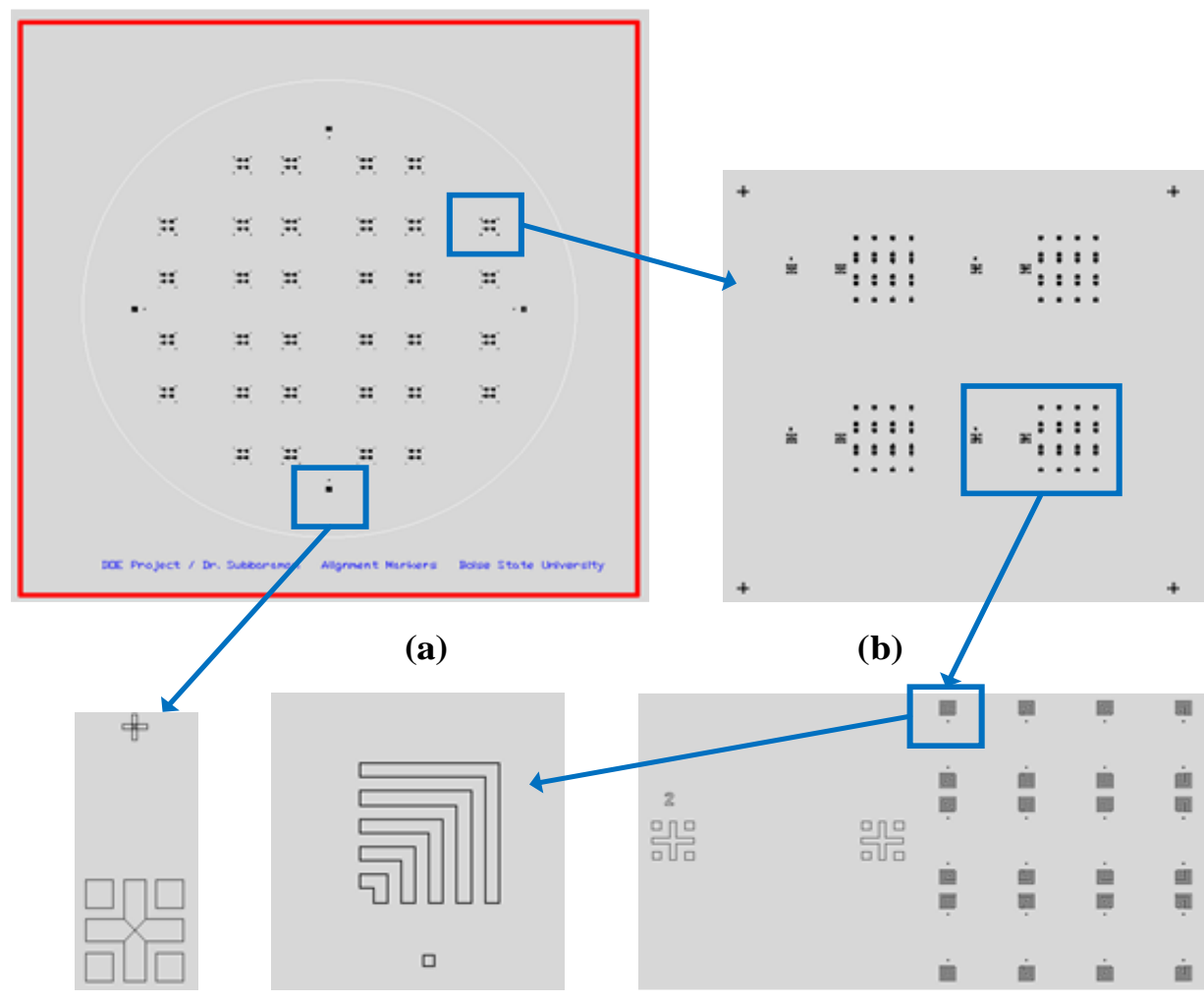

(c)

(d)

(e)

Figure 6.13: CAD image of photo mask with registration marks, a) photo-mask layout, b) Registration marks on one chip, c) global mark, d) chip mark, e) inset of marks in one array waveguides.

In this design, four global registration marks are placed on the outer edges of 
the sample along the $x$ and $y$ axis as shown in Figure. 6.13c. Also for each subfield $(200 \mu m \times 200 \mu m)$ shown in Figure. 6.14a, four chip marks are dedicated as shown in Figure. 6.13(d,e). The system usually detects four global marks to calculate the expansion, contraction, rotation, and shift amount of the substrate and correct the system with the obtained values. After global-mark detection, the system also detects the four chip mark positions near the writing region to correct the deflection gain and rotation of the main and sub-deflectors near the writing region, and writes the pattern according to the shape. The pattern can be aligned to the existing marks without exposing the writing area by using the alignment program in NPGS. There are two windows, large coarse alignment and fine alignment windows in this program as shown in Figure. 6.14. 


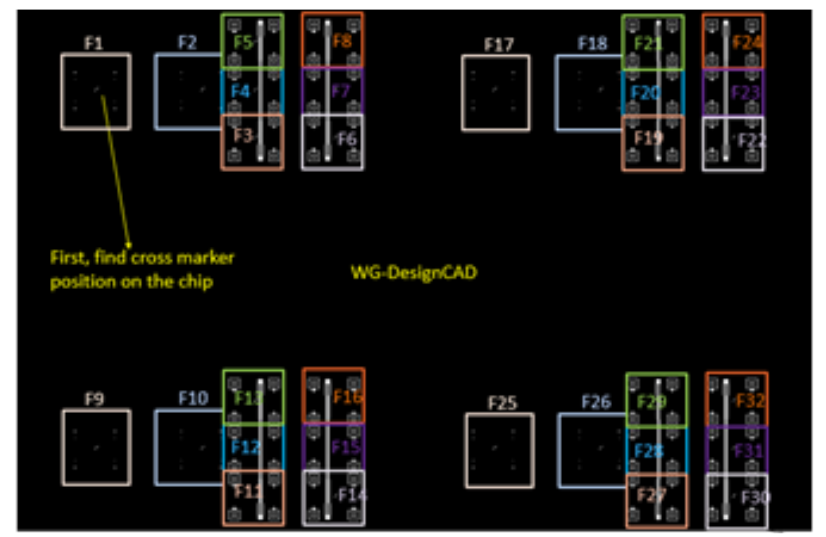

(a)

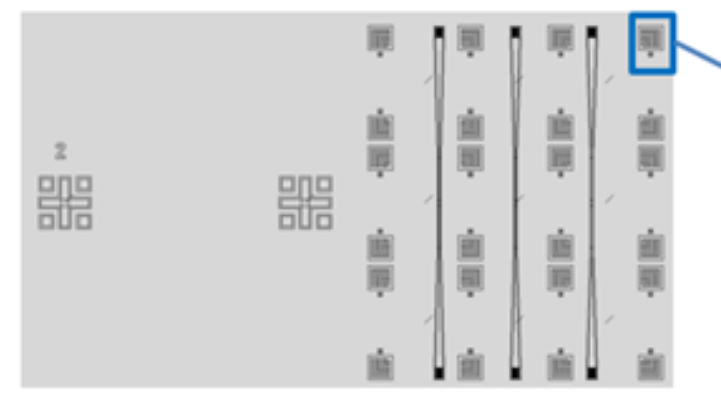

(b)

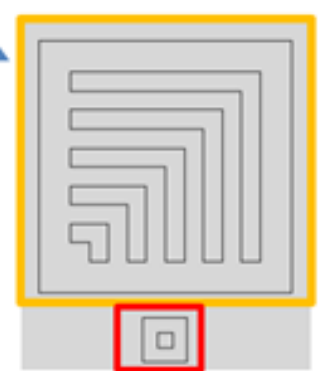

(c)

Figure 6.14: a) Fracture writing field to $200 \mu m \times 200 \mu m$ sub-fields, b) CAD design of array waveguides, c) Coarse and fine registration chip marks

After alignment, the program calculates a general transformation matrix to correct the $x$ and $y$ magnification error of the microscope, sample rotation and offset. This will enhance the alignment accuracy. The photo-mask was used in patterning the waveguide with grating coupler. In the next few sections, we discuss the patterning of individual parts forming the integrated waveguide system. 


\subsubsection{Grating coupler terminated silicon waveguide fabrica- tion using registration marks}

To achieve high resolution grating couplers in the fabrication, the waveguide grating coupler designs can be patterned in each writing field using the stitching method. In order to write the markers on the SOI wafer, a $220 \mathrm{~nm}$ top silicon layer and a 3 $\mu m$ buried oxide layer, is spin coated with ZEP520A photoresist. In order to have high resolution, high sensitivity, and durability in process, a positive beam resist ZEP520A was chosen for the process. It provides a high resolution, high sensitivity and is durable during the etching process which will be used to define silicon waveguide. Then, the photolithography was performed used to pattern the registration markers. The exposed wafer is developed and the design is transferred to Si layer using Reactive-Ion Etching (RIE). The next step in the process is to remove the top layer of photoresist with use of ZEP-N50 stripper and an asher, a low powered $\mathrm{O}_{2}$ RIE. The registration marks are thus created on the SOI wafer. The wafer with markers on it can be used in further fabrication steps. Next step is preparing the wafers with markers patterned can be used in further fabrication steps. Next step is preparing the wafers with markers on it for EBL to pattern the waveguide designs. The wafer was spin coated with ZEP520A with dilution rate of 1.7 (see the Appendix. B. In NPGS software the field is fractured to the $200 \mu m \times 200 \mu m$ subfields. The exposure conditions are: Beam area dose $=75 \mu C / \mathrm{cm}^{2}$, volatge $=30 \mathrm{kV}$, beam current $=$ $55 \mathrm{pA}$. The wafer is aligned to the stage using alignment marks and AL alignment program in NPGS software. The length of waveguide section was chosen as $150 \mu \mathrm{m}$ in order to have enough space to deposit the ChG. The pattern was transferred to the SOI through EBL system, followed by the RIE. The microscope images of fabricated 
devices are shown in Figure. 6.15. Three waveguides are placed about $127 \mu \mathrm{m}$ apart from each other. This distance is selected based on the pitch to pitch distance of the 1D fiber array purchased from Fiberguide Industries.

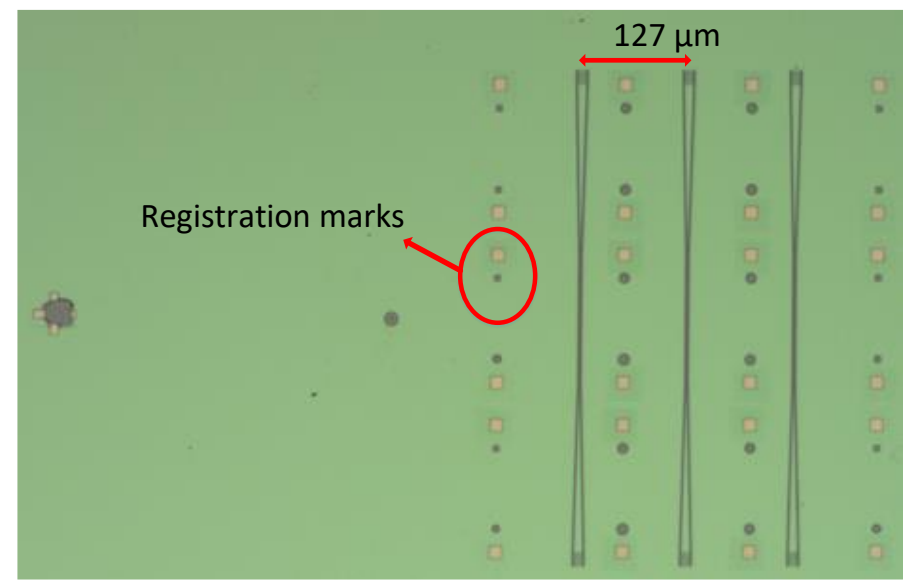

(a)

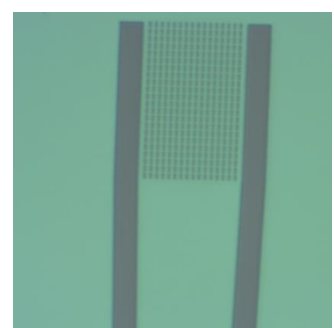

(b)

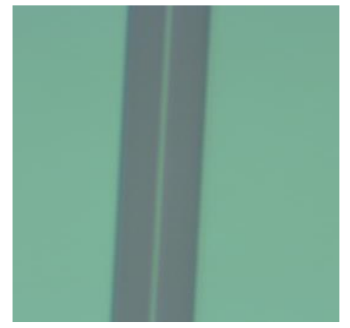

(c)

Figure 6.15: Microscope image of fabricated array of subwavelength grating coupler ended waveguide, a) array of waveguide structure, b) waveguide, c) grating coupler.

\subsection{S-bend Waveguide Fabrication}

Bend waveguides are a key element in miniaturization of photonic integrated circuits (PIC) and optical interconnections. When a number of optical components integrated in one system, bending waveguides offers best solutions for developing high 
density PIC [128]. S-bend waveguides are used to connect two straight integrated optics waveguides that are offset with respect to each other or to connect integrated optical devices such as directional couplers, optical switches and array waveguide gratings [129]. We have fabricated S-bend waveguide based temperature sensor for three reasons: a) ensure a working device, b) eliminate risks by grating coupler fabrication errors, c) integrated the proposed sensor in a PIC.

We fabricated fabricated S-bend waveguide based temperature sensor on SOI wafer with $220 \mathrm{~nm}$ device layer and $3 \mu \mathrm{m}$ Box oxide layer. This waveguide can be easily fabricated and tested using edge coupling set up. The AutoCAD design of the S-bend waveguide is shown in Figure. 6.16.

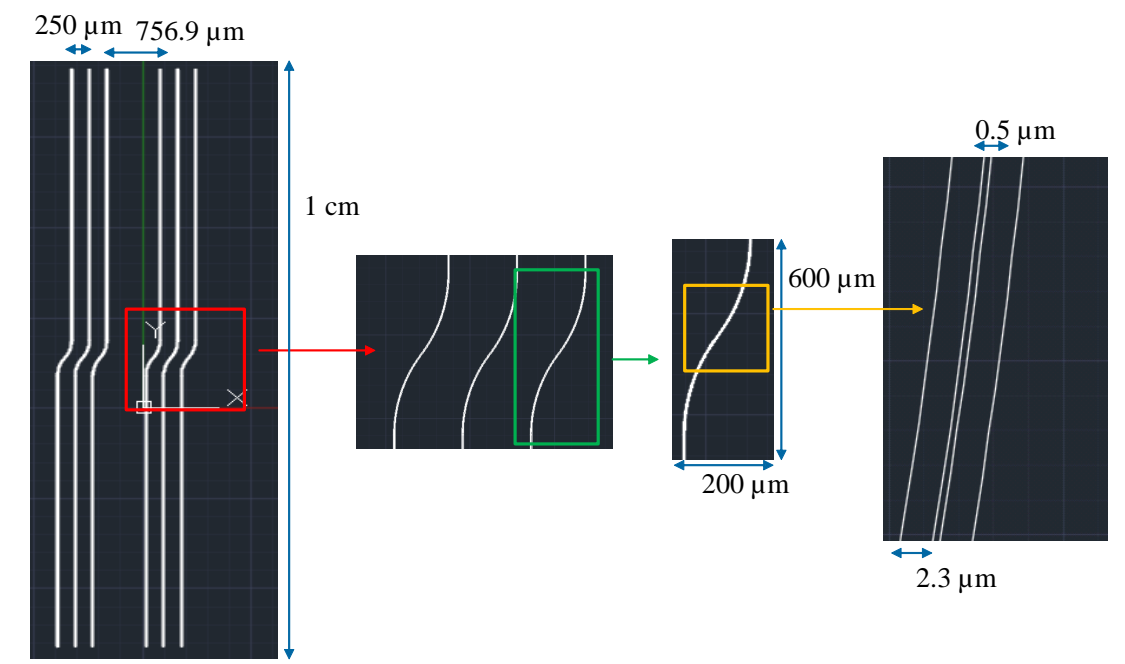

Figure 6.16: CAD design of array S-bend waveguides

The waveguide width is selected $500 \mathrm{~nm}$ and $2.3 \mu \mathrm{m}$ border with $500 \mu \mathrm{m}$ curvature radius which shows single mode characteristic. The fabrication process for the waveguides is similar to that described in Section 6.2 for the grating coupler based devices. Hence for clarity, only the SEM image of fabricated an array of S-bend waveguides is 
shown in Figure. 6.17.
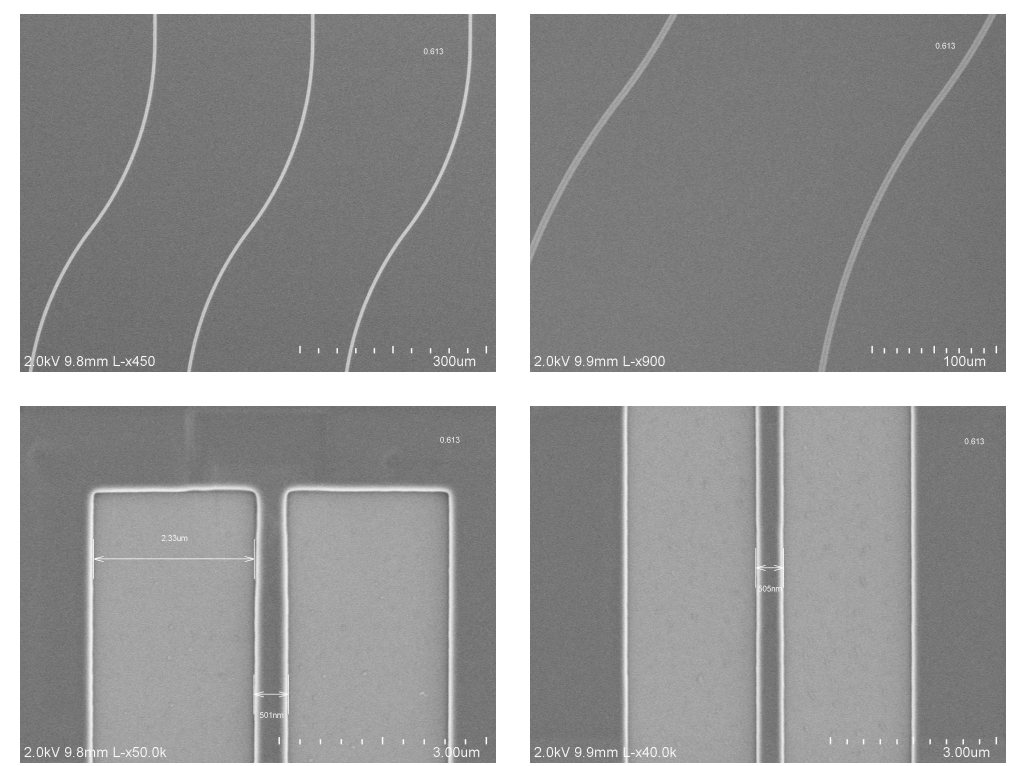

Figure 6.17: SEM image of fabricated an array of S-bend waveguides.

\subsection{In-house Synthesized Ink Covering of the}

\section{Silicon Waveguide}

We fabricated a photo-mask with a tiny hole with the size equal to waveguide length on a thick semi-transparent Kapton substrate. Instead of using a laser engraver to form a pinhole, a pin was used to create a pinhole on the shadow mask. Then, we manually aligned the hole with waveguide section under a microscope. To cover the waveguide with a in-house synthesized ink thermal evaporation method was selected. We followed the same thermal evaporation condition ( $10^{-6}$ mbar vacuum and evaporation rate of $0.35 \AA / \mathrm{s}$ that we mentioned in Section 4.1.2. Figure. 6.18 shows the process of covering waveguide sections with fabricated shadow masks. A microscope 
image of a waveguide covered with $\mathrm{Ge}_{40} \mathrm{Se}_{60}$ is shown in Figure. 6.19.

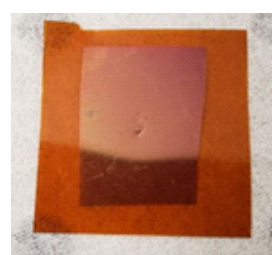

Shadow mask

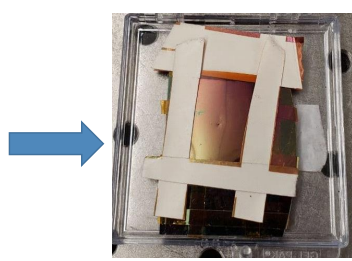

Shadow mask covers the sample

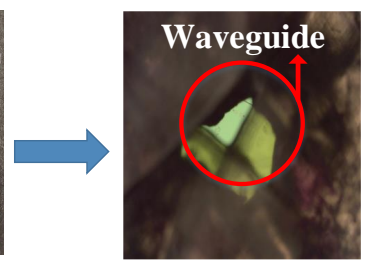

Waveguide image under a microscope through shadow mask

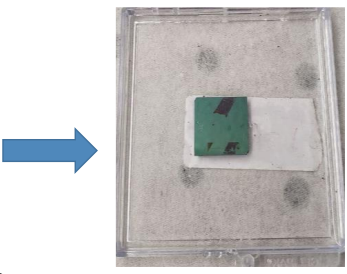

Sample after thermal deposition of $\mathrm{ChG}$

Figure 6.18: A shadow mask fabricated at Boise State University on Kapton substrate for direct chalcogenide glass evaporation over the waveguides.

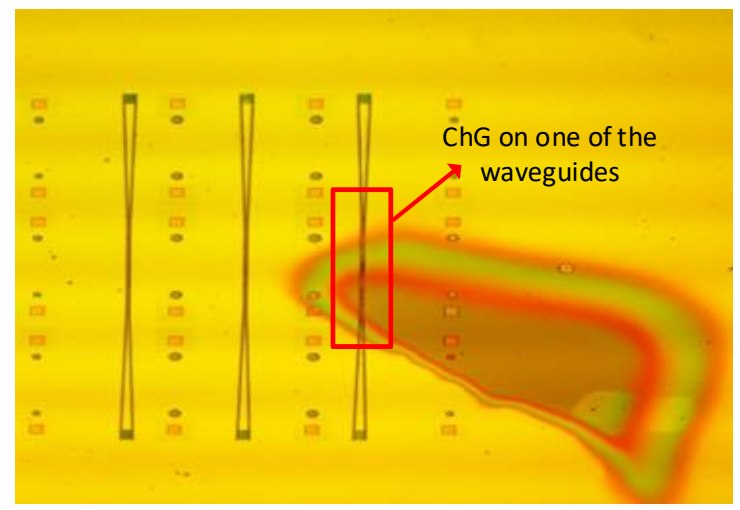

Figure 6.19: Chalcogenide glass covered Si waveguide.

\subsection{Experimental Setup}

The schematic and actual pictures of the waveguide testing setups (grating coupler and edge coupling) we built at Boise State University are shown in Figure. 6.20. In the grating coupling set-up as shown in Figure. 6.20(b,c). 


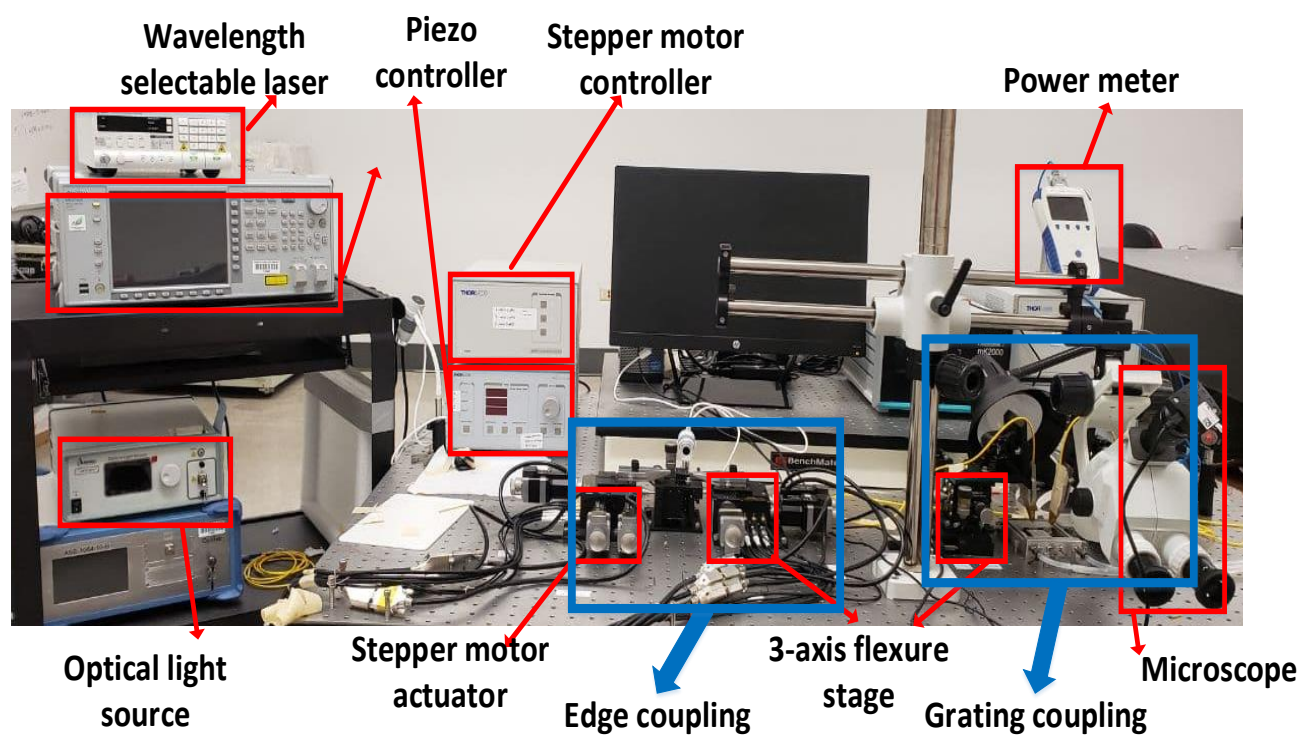

(a)

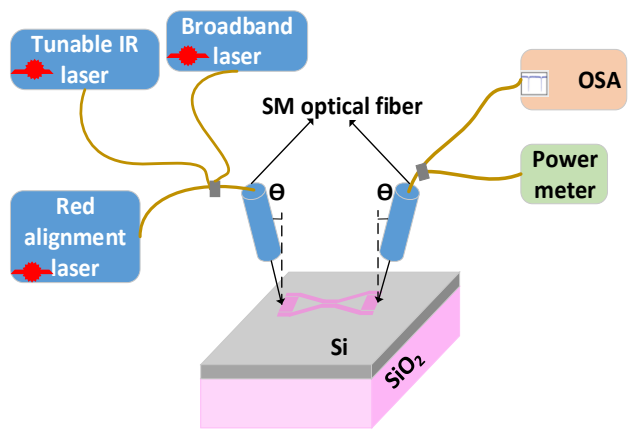

(b)

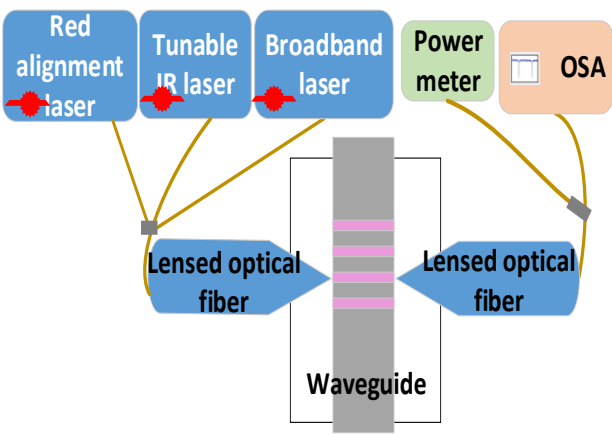

(d)

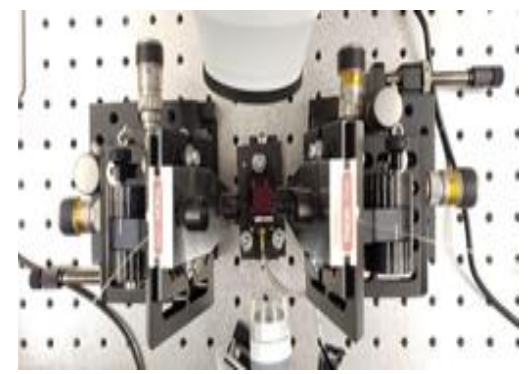

(c)

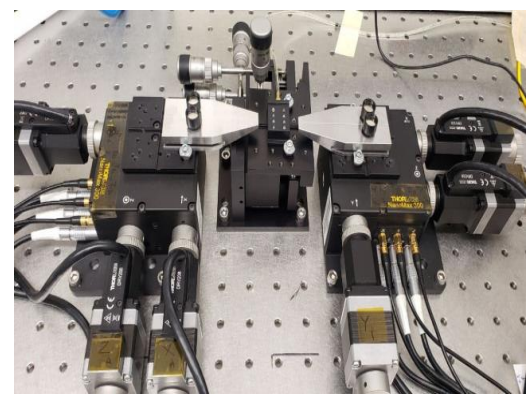

(e)

Figure 6.20: a) Waveguide characterization set up at Boise State University, b) grating coupler testing setup schematic, c) close view of grating coupler testing setup, c) edge coupling testing set up schematic, d) close view of grating coupling testing set up. 
The device is characterized by measuring the fiber-waveguide-fiber insertion loss coupled via a pair of grating couplers connected by waveguide. Single mode fiber (SMF) are stripped of its coating and cleaved to form fiber probe. Cleaved input and output fibers mounted on two wedges which are mounted on precision 3-axis translation stages. These fibers are tilted at $10^{\circ}$ from the grating couplers. The tilt angle can be adjusted from $0^{\circ}$ to $90^{\circ}$. For our grating coupler design, a tilt angle of $10^{\circ}$ provided the highest coupling efficiency. The fiber positions are controlled by precision XYZ micrometer stage for maximum transmission. To center the fiber to the grating coupler area with small distance above the surface, two cameras-one providing a side view and another providing an image at $45^{\circ}$ angle are used to visually aid alignment. We used red light source to align the fibers to the grating couplers as shown in Figure. 6.21.

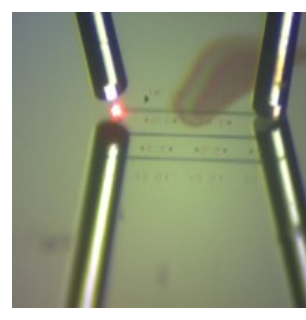

(a)

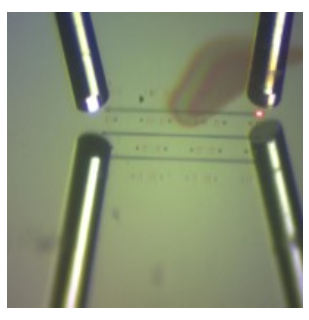

(b)

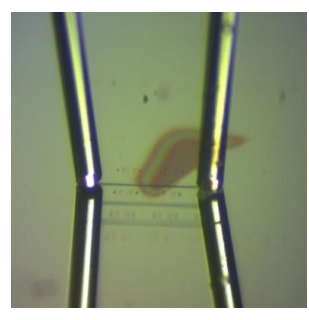

(c)

Figure 6.21: a) Aligning the fiber with the input grating coupler using red light, b) aligning the fiber with the output grating coupler using red light, c) temperature sensor device under temperature test.

The input fiber is connected to a broadband laser source that covers $\mathrm{C}+\mathrm{L}$ bands (Amonics, ALS-CL-20-B). The light from the input optical fiber is coupled to the chip via the input subwavelength grating coupler, injecting the light into a silicon wire interconnecting waveguide, after which it is coupled out of the chip to the output optical fiber through another identical subwavelength grating coupler structure. 
A length of $250 \mu \mathrm{m}$ is utilized to connect $10 \mu \mathrm{m}$ wide grating couplers to the $450 \mathrm{~nm}$ width waveguide. The output fiber is connected to a power detector and optical spectrum analyzer (OSA). We used a programmable heat stage (INSTEC, HCS621G) in the grating coupling set-up to test the real-time temperature response of the Si waveguide grating based temperature sensor as shown in Figure. 6.22. The temperature was increased from room temperature $\left(\sim 25^{\circ} \mathrm{C}\right)$ to $600{ }^{\circ} \mathrm{C}$ in $10^{\circ} \mathrm{C} / \mathrm{min}$ increments.

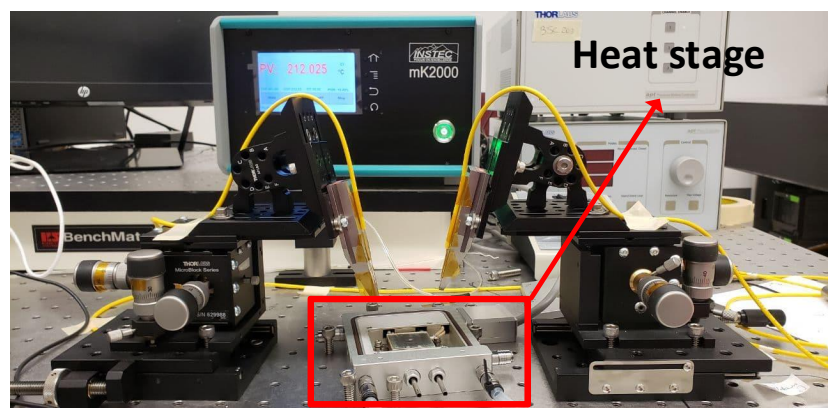

Figure 6.22: Testing set-up for characterizing the temperature response of grating terminated silicon waveguide based temperature sensor.

Also, an edge coupling set-up was prepared to couple light through S-bend waveguide as shown in Figure. 6.20(d,e). In this method, the sample is cleaved and waveguide cross-sections are exposed on the both ends. Lensed optical fiber focuses the incoming light into the waveguide end-facet. The lensed fiber reduces the beam spot size to $2-3 \mu \mathrm{m}$ to improve the coupling efficiency. Another lensed fiber is used at output stage to collect the transmitted power. The output power can be measured using power meter or OSA. By checking the fiber tip and waveguide edge under microscope, the alignment of the waveguide and fiber is optimized. The optimized fiber tip position should be able to collect the transmitted power from Si waveguide not from the substrate on silicon handling layer. There are some limitations in coupling 
the light with an edge coupling method- a) the sample should be precisely cleaved, b) the waveguide should be sufficiently long enough to ensure a good cleaving, c) the loss is high and can be reduced by use of focused beam spot and tapered waveguide, d) high power input light source is required to have output power above the noise level, e) alignment of fiber tip with input/output power is time consuming, and f) the lensed fiber tip should be adjusted within proper distance from cleaved end of waveguide in order to have high transmitted power.

\subsection{Results and Discussion}

Before covering the waveguide with one of the in-house scythed inks, we characterized a reference waveguide to confirm that the fabricated device works properly. The measured fiber-to fiber coupling efficiency normalized to the light source is shown in Figure. 6.23.

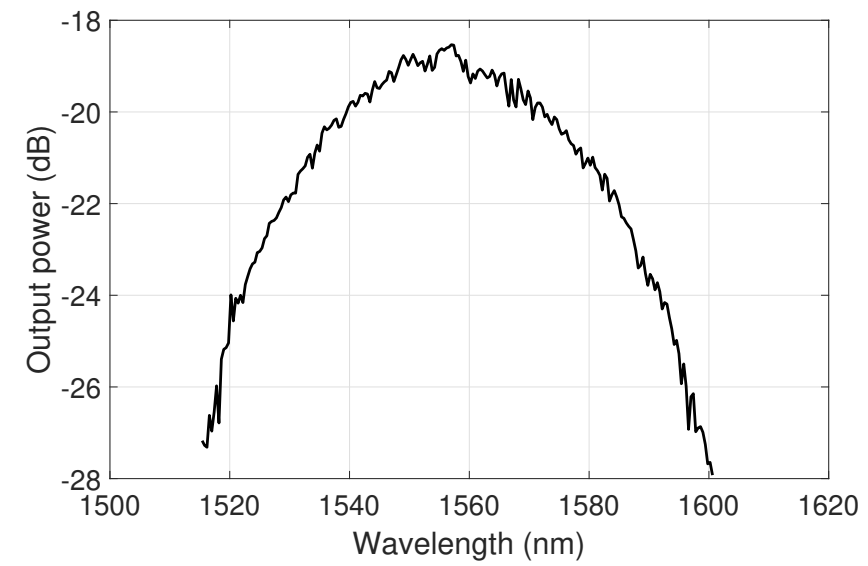

Figure 6.23: Measured transmission spectrum from the fabricated device without ChG cover layer on the waveguide.

The maximum normalized transmitted power measured was $-18.53 \mathrm{~dB}$. By considering negligible loss in taper and waveguide loss, each grating coupler has $\sim 8 \mathrm{~dB}$ 
loss. The Fabry-Perot ripples near the peak indicates very low reflection. The $1 \mathrm{~dB}$ and $3 \mathrm{~dB}$ bandwidths are $24 \mathrm{~nm}$ and $51 \mathrm{~nm}$, respectively. The designed peak wavelength is shifted from $1550 \mathrm{~nm}$ wavelength to $1557 \mathrm{~nm}$ due to fabrication error during patterning with EBL. This error varies the size of features in grating couplers. It also slightly changes the filling factor. Increasing the filling factor, increases the grating propagation constant, weakening the grating and consequently shifting the peak to longer wavelengths. Also, fabrication error causes extra loss in grating coupler.

To measure the real-time temperature response of the sensors, we have tracked the heat stage temperature as a function of the time as shown in 6.24.

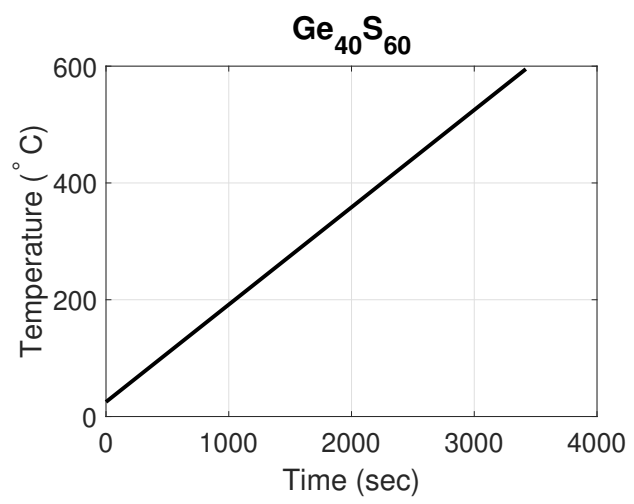

Figure 6.24: Temperature of heat stage as a function of time.

The normalized measured transmitted power (dotted black curve) as a function of time for a composition $\mathrm{Ge}_{40} \mathrm{~S}_{60}$ is shown in Figure. 6.25. The normalized simulated transmitted power with extracted refractive index profile from the particular ChG as a function of time is also plotted (solid red curve). It can be seen from the figure that the measured and simulated results match very well. Measurement result this figure shows that at higher temperature $\left(>500{ }^{\circ} \mathrm{C}\right)$ the transmitted power increases. One reason is that $\mathrm{ChG}$ was evaporated i.e the $\mathrm{ChG}$ layer from top surface of the $\mathrm{Si}$ 
waveguide deteriorates at high temperature. Then, the plasmonic waveguide changed to dielectric waveguide which confine the light in Si core. Protecting ChG with spinon-glass can avoid the evaporation.

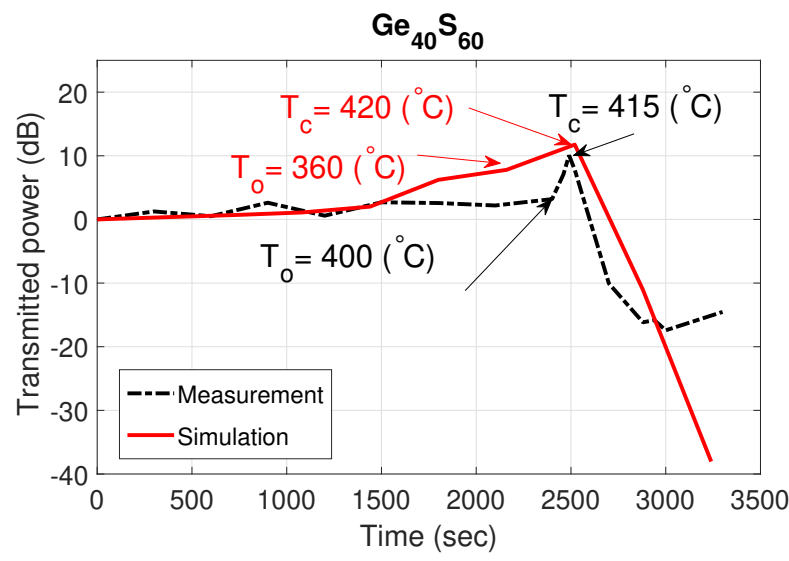

Figure 6.25: Simulated and measured normalized transmitted power as a function of time with $\mathrm{Ge}_{40} \mathrm{~S}_{60}$ covered silicon waveguide based temperature sensor.

As the proposed temperature sensor works on principle of phase change of ChG material, which is highly temperature dependent, sudden changes in the transmitted power is observed, as expected. We plotted slope as a function of time to extract the sudden change in the transmitted power as shown in Figure. 6.26. 


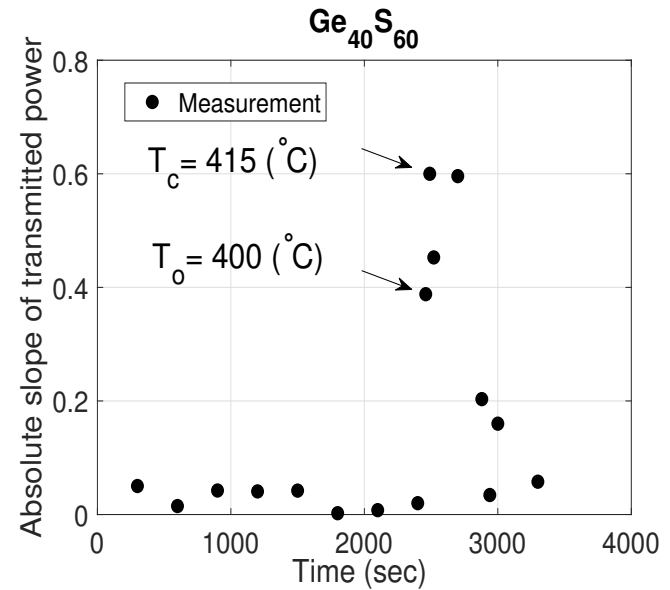

(a)

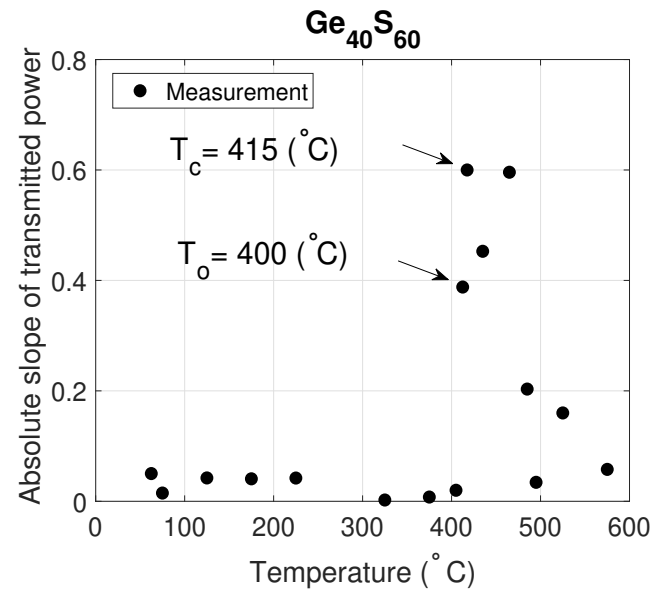

(b)

Figure 6.26: Temperature response of $\mathrm{Ge}_{40} \mathrm{~S}_{60}$ covered silicon waveguide based temperature sensor, a) absolute slope of transmitted power as a function of time, b) absolute slope of transmitted power as a function of temperature.

The big growth of absolute slope of the transmitted power between $\mathrm{T}_{o}$ and $\mathrm{T}_{c}$ due to large changes in structural reorganization occurs from the amorphous phase to the crystalline phase where the first crystallization is at $\mathrm{T}_{o}$ and the full crystallization occurs at $\mathrm{T}_{c}$. Two peaks corresponds to the onset and crystallization temperatures of $400{ }^{\circ} \mathrm{C}$ and $415{ }^{\circ} \mathrm{C}$ obtained from monitoring the temperature from the heat stage, which is close to the expected temperatures $360{ }^{\circ} \mathrm{C}$ and $420{ }^{\circ} \mathrm{C}$. The second highest peak is expected to be the second crystallization temperature of the $\mathrm{Ge}_{40} \mathrm{~S}_{60}$ as shown in Table. 3.1. Thus, this experiment confirms the functionality of our proposed compact Si waveguide:ChG based temperature sensor which help us with detection of real-time temperature inside a high temperature environment.

\subsection{Summary}

Over the course of this chapter, we have established the EBL process flow that enables the fabrication of the Si waveguides. Also, the method to cover the waveguide with 
ChG using shadow mask is described. In order to characterize the performance of the proposed sensor, the testing setup is built. The real-time temperature response of the sensor is measured and the result is compared with the simulation result. The temperature response of the sensor is in good agreement with the simulation. 


\section{CHAPTER 7:}

\section{FUTURE RESEARCH}

There are some immediate paths for extending the research presented in this dissertation.

\subsection{Packaging Details for Waveguide based Temperature Sensors}

In order to realize practical application of silicon waveguide based temperature sensors, the circuit must be packaged with optical single-mode fibers coupling. In order to minimize coupling loss caused by the second-order reflection, a grating coupler was designed to couple the light in/out with an angle $(\theta)$ off vertical direction. In general, in packaging of fiber to waveguide grating coupler, the fiber must be polished with an angle $\left(\theta_{\text {fiber }}\right)$ that launches the light with totally reflected light into the grating coupler follows Equation. 7.1. The total internal reflection(TIR) relation in Equation. 7.2 shows the angle which TIR occurs [130]. This equation also shows the limit of angles that can be used. This TIR equation is true only when the fiber facet is surrounded by air. It is desirable to maintain a planar profile so as to decrease the overall size of the package. Angled polished fiber should be placed into a silicon V-groove carrier. A red-light laser source can be used to align the fiber to a grating coupler. The

alignment tolerances can be performed using a 3-axis flexure. After completing the 
alignment, the fiber should be cured in the V-groove with UV-cured index matching epoxy. Two fibers in V-grooves should be align over the input and output grating couplers. Also, matching epoxy can be used to bond the fiber to the chip, as shown in Figure. 7.1. The distance between the fiber and the grating couplers should be studied to have highest transmitted power. In array of sensors the pitch between adjacent grating couplers must be a multiple of $127 \mu \mathrm{m}$ or $250 \mu \mathrm{m}$ throughout the whole chip, when choosing an array fiber to couple the light to an array sensor. To complete the packaging the chip should be mounted on a submount and attach the V-grooves to the same.

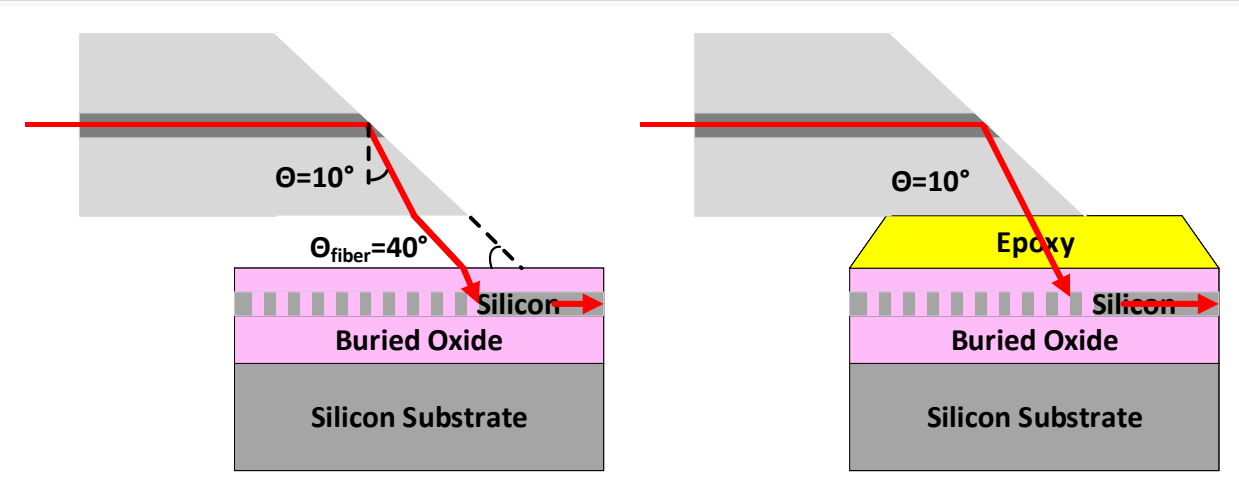

Figure 7.1: Using refractive index matching UV-cured epoxy to bond fiber to the Si waveguide device.

$$
\begin{gathered}
\theta_{\text {fiber }}=\frac{90^{\circ}-\theta}{2} \\
\theta_{T I R}=\operatorname{Sin}^{-1}\left(\frac{1}{1.4682}\right)=42.930^{\circ}<\left(90^{\circ}-\theta_{\text {fiber }}\right) .
\end{gathered}
$$

Proper packaging of optical fiber can extend their application in harsh environments including high temperature and high radiation. The fibers may survive in these 
environments but their fragility requires them to be properly protected. There are several possible solutions. One potential solution is embedding optical fibers in a metal matrix and bond the fibers to a metal substrate using epoxy [131-133]. However, de-bonding becomes an issue at higher temperatures in epoxy-based bonding techniques. To overcome this issue, a low-temperature ultrasonic additive manufacturing (UAM) process can perform to bond fibers embed fibers directly within the metal matrix (aluminum and nickel) [134-137].

Recently, ceramic materials such as silicon carbide ( $\mathrm{SiC})$ due to their high-temperature strength retention approaching or exceeding $1000{ }^{\circ} \mathrm{C}$, stability under neutron irradiation, and low neutron absorption are attractive for packaging optical sensors for nuclear applications [138]. A binder jet printing process (3D printer) can be used to fabricate $\mathrm{SiC}$ components with complex geometries. The cavity in which the fiber sensor should be inserted can be printed using a 3D printer and then fix the fibers' location using chemical vapor infiltration process [139].

Another solution to package optical fibers is using a printer to embed the fibers in ceramic components. An aqueous paste of ceramic particles can be extruded through a moving nozzle to build the substrate layer-by-layer. Then, place the array of fibers in their predetermined locations. Finally, continue the printing process and cover the fibers with the remaining layers of ceramic. To ensure the fabrication process is flawless, scanning electron microscopy can be used [140]. 


\subsection{Integration of Arrays Si waveguide:ChG based}

\section{Temperature Sensor}

Arranging the single $\mathrm{ChG}$ top coated waveguides in an array structure, as shown in Figure. 7.2, and monitoring the transmitted power from each waveguide will help with the real-time detection of several temperatures inside extreme environments, thus allowing the user to map out the temperature profile. As mentioned before, each of the synthesized $\mathrm{ChG}$ compositions has a specific crystallization temperature which allows for accurate monitoring and recording of real-time temperature profile within a desired environment.
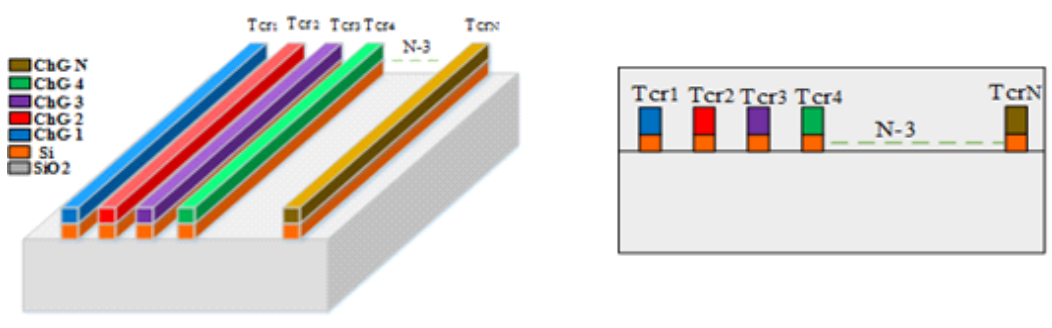

(a)

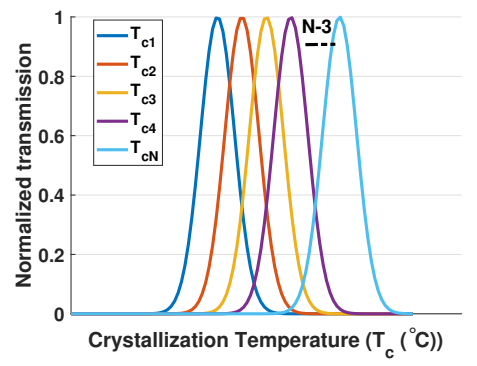

(b)

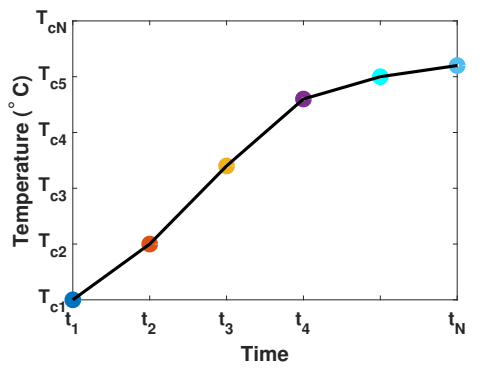

(c)

Figure 7.2: a) Waveguide array with different ChG topping, b) output power versus temperature from the ChG covered Si waveguide, c) Temperature trend as a function of time using array structure. 
A schematic of a multi-point temperature sensors through incorporation of different ChG glasses with varying $T_{c}$ on different silicon waveguides is shown in Figure. 7.3.

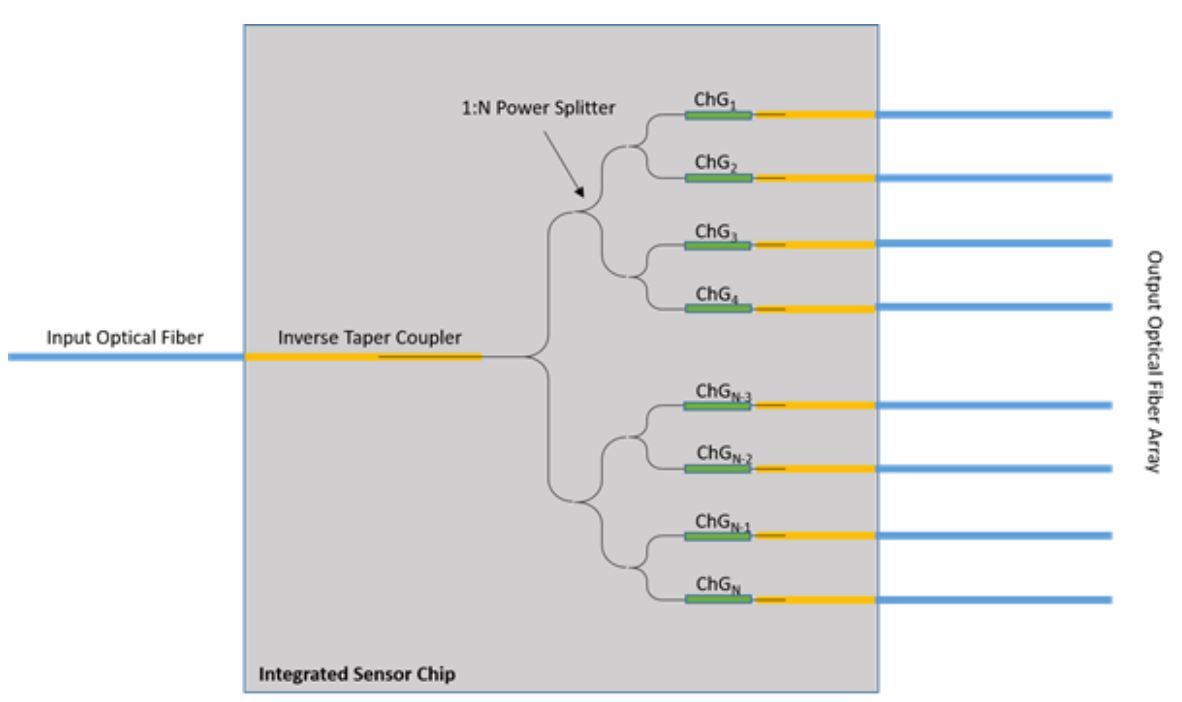

Figure 7.3: A multi-point temperature sensor chip incorporating inverse taper fiber couplers, 1-to-N power splitters, waveguides coated with different ChG glasses with varying $T_{c}$, and input and output radiation hardened optical fibers.

The sensor chip architecture consists of (1) a silicon waveguide array, with a different chalcogenide top cladding (with a slightly different $T_{c}$ ) on each waveguide, $(2)$ a 1-to-N power splitter to distribute input light power to $\mathrm{N}$ sensor waveguide channels, and (3) inverse taper couplers for coupling light from optical fiber into the input waveguide and coupling light out of the output waveguide array into radiation hardened optical fibers. Inverse taper enable efficient light coupling ( $<1 \mathrm{~dB}$ per facet loss) from optical fiber into the silicon waveguide, and the input light is then distributed to the different sensor channels using a 1-to-N power splitter. The radiation hardened optical fibers guide light to (from) the external environment from (to) the sensor chip. A gold coating on the fiber commercially available in Fiberguide Industries (Caldwell, 
ID) will enable operation $>700{ }^{\circ} \mathrm{C}$ and improve the Rad Hard characteristics of these fibers.

\subsection{Sensitivity Analysis of Si waveguide:ChG based Temperature Sensor}

The sensor is designed to detect the temperature up to $600{ }^{\circ} \mathrm{C}$. A Gaussian light beam emitted from a laser light source is applied at the input of the waveguide and is detected using a detector with the monitor placed at the output of the waveguide. The sensing principle is based on the changes in refractive index of ChG coating the waveguide when the temperature varies. In other words, $n_{C h G}$ is a function of the temperature. We can calculate the the sensitivity $\left(S_{T}\right)$, which is a fundamental parameter in evaluating the performance of the temperature sensor. In order to study the sensitivity of the proposed sensor, the wavelength shift over a temperature change during the thermal test should be tracked. The sensitivity can be expressed as:

$$
S_{T}=\frac{\Delta \lambda}{\Delta T}
$$

where $\Delta \lambda$ is changes in the resonant wavelength, and $\Delta T$ is the changes in the tem-

perature. $S_{T}$ is calculated in term of $p m /{ }^{\circ} \mathrm{C}$, and should be as high as possible. Figure. 7.4 shows schematic of the transmission spectra as a function of the wavelength with varying the temperature. Then, by using FDTD method or interpolation methods, the resonant wavelength peak with respect to the temperature can be indicated, as shown in Figure. 7.4. By calculating Equation. 7.3, the sensitivity of the device can be obtained. 


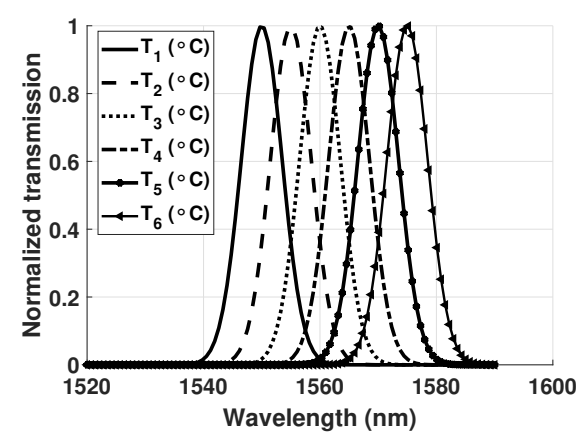

(a)

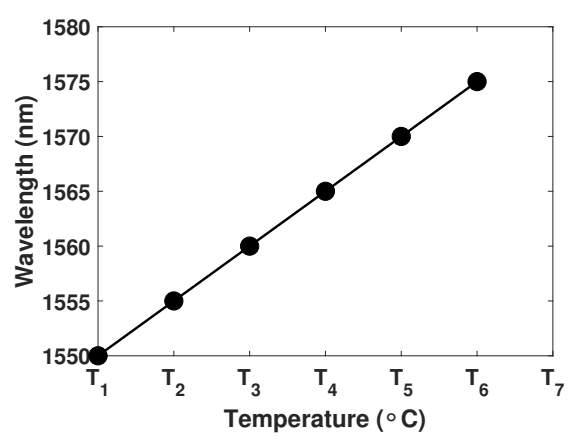

(b)

Figure 7.4: a) Transmission spectra of the temperature sensor for different temperatures, b) Linear relationship between the temperature and the resonance wavelength. 


\section{CHAPTER 8:}

\section{CONCLUSION}

In this dissertation, two optical waveguide based high temperature sensors are demonstrated.

Firstly, an optical waveguide based temperature sensor is demonstrated to enable measuring real-time temperature within extreme environments. The optical fiber with coated end facet with ChG glass is modeled using PhotonDesign software. The working principle of the sensor depends on the phase transition of the ChG deposited on optical fiber tip from amorphous to crystalline phase at well-defined temperatures, thereby causing rapid changes in the complex refractive index of the material. These changes in the optical properties lead to sudden changes in the reflected intensity that can be used to detect the ambient temperature inside nuclear reactors. According to the high temperature sensitivity of the $\mathrm{ChG}$, the proposed sensors can record accurate temperatures within the proposed environment. The idea of these proposed sensors is to utilize the big difference in optical performance such as transmission or absorption power as the ChG glass reaches a particular transition temperature. Besides, the ability to revert the crystalline state to an amorphous state by application of an electric field facilitates multiple-time use of the sensors. To fabricate the proposed

temperature sensor, gold from the tip of the optical fiber was stripped using Aqua regia and then the tip was cleaved using a standard cleaving tool. The cleaved optical fiber 
tip was capped with one of six different compositions of in-house synthesized ChGs using thermal evaporation and dip coating methods. The temperature response of fabricated sensors was characterized using a testing setup at Boise State University. The reflected power from the capped tip of fiber was monitored by increasing the temperature from RT to $600{ }^{\circ} \mathrm{C}$. To track the temperature, we fabricated an array of sensors that can effectively track temperature from $440-600{ }^{\circ} \mathrm{C}$. The measured results were matched well with simulation results. Thus, the experimental results reveal that these sensors are promising candidates due to reliability, small size, light weight sensor network for use within high temperature and high-radiation environments. The material's studies reveal that the occurrence of big compositional and structural changes in Ge-rich compositions after crystallization leads to better-expressed device effects, which is important when considering material choices for building devices. These compositions offer phase change temperatures close to those of chalcogen-rich materials.

Secondly, a Si:ChG integrated waveguide sensor is demonstrated applicable for a wide range of temperature sensing in extreme environments. The working principle of the sensor depends on the transformation of the glass from amorphous to crystalline phase at a specific crystallization temperature. According to the high loss of plasmonic waveguide in fundamental modes of propagation due to the creation of plasmonic modes at the interface between silicon waveguide and $\mathrm{ChG}$ glass cladding, a high extinction coefficient can be obtained with a very short length of the waveguide. Therefore, by monitoring the output power from the waveguide as a function of temperature, the exact determination of the instant at which the reactor crosses the phase transition temperature can be achieved. The proposed silicon waveguide 
based plasmonic sensors for sensing temperature inside the nuclear reactor are simulated using PhotonDesign software. The simulation data gave us the optimum device dimension on the SOI wafer. The Si waveguide design was patterned on an SOI wafer using EBL. To achieve high-resolution patterning in EBL, I used the stitching method. After patterning the waveguide grating structure, the design was transferred to the Si device layer using RIE. We fabricated a shadow mask with a tiny hole with the size equal to a waveguide and manually aligned the hole with a waveguide section under a microscope. We used the thermal evaporation method to cover the waveguide with an in-house synthesized ink. The temperature response of the fabricated sensor was tested using a testing setup built in Dr. Subbaraman's lab. The measurement result was in good agreement with the simulation results. We obtained rapid changes in transmitted power beyond crystallization temperature of in use chalcogenide glass which confirms the transformation of a dielectric waveguide to plasmonic waveguide by changing the ChG phase from amorphous to crystalline with increasing the temperature. The measured onset and crystalline temperatures are close to the values that we expected from studying thin film. The simulation and measurement results match very well.

This remarkable property, together with CMOS-compatible fabrication of the silicon device and optical fibers promises a very reliable, low-cost, and reusable sensor network. 


\section{BIBLIOGRAPHY}

[1] G. Locatelli, M. Mancini, and N. Todeschini, "Generation IV nuclear reactors: Current status and future prospects," Energy Policy, vol. 61, pp. 1503-1520, Oct. 2013.

[2] B. Kallman, "The very high temperature reactor," Web, MAR, 2013.

[3] J. E. Kelly, "Generation IV international forum: A decade of progress through international cooperation," Prog. Nucl. Energy, vol. 77, pp. 240-246, Nov. 2014.

[4] P. Pursula, I. Marttila, K. Nummila, and H. Seppä, "High frequency and ultrahigh frequency radio frequency identification passive sensor transponders for humidity and temperature measurement within building structures," IEEE Trans. Instrum. Meas., vol. 62, no. 9, pp. 2559-2566, May 2013.

[5] Y. Huang, Z. Zhou, Y. Zhang, G. Chen, and H. Xiao, "A temperature selfcompensated LPFG sensor for large strain measurements at high temperature," IEEE Trans. Instrum. Meas., vol. 59, no. 11, pp. 2997-3004, May 2010.

[6] H. Hashemian, "Nuclear power plant instrumentation and control," Nuclear Power-Control, Reliability and Human Factors, InTech, pp. 49-66, 2011. 
[7] J. Daw, J. Rempe, D. Knudson, T. Unruh, B. Chase, K. Davis, and A. Palmer, "Temperature monitoring options available at the idaho national laboratory advanced test reactor," AIP, vol. 1552, no. 1, pp. 970-975, Sept. 2013.

[8] H. Hashemian, "Maintenance of process instrumentation in nuclear power plantspringer-verlag," Berlin Heidelberg, 2006.

[9] H. Hashemian and K. Petersen, "Achievable accuracy and stability of industrial RTDs," Temperature: Its Measurement and Control in Science and Industry, vol. 6, no. 1, pp. 427-432, 1992.

[10] P. Tsvetkov, Nuclear power: Control, reliability and human factors. BoDBooks on Demand, 2011.

[11] T. Kuglestadt, "Semiconductor temperature sensors challenge precision RTDs and thermistors in building automation," Texas Instruments: Application Report: SNAA267-04, pp. 2-10, 2015.

[12] Standard Guide for Use of Melt Wire Temperature Monitors for Reactor Vessel Surveillance, E 706 (IIIE). West Conshohocken, PA: ASTM, 2006. [Online]. Available: https://cds.cern.ch/record/1460534

[13] J. L. Rempe, D. L. Knudson, J. E. Daw, T. Unruh, B. M. Chase, J. Palmer, K. G. Condie, and K. L. Davis, "Enhanced in-pile instrumentation at the advanced test reactor," pp. 1-10, Apr. 2011.

[14] M. Scervini, C. Rae, and B. Lindley, "Transmutation of thermocouples in thermal and fast nuclear reactors," 3rd International Conference on ANIMMA, pp. 1-8, Jun. 2013. 
[15] R. E. Bentley, Handbook of temperature measurement: The theory and practice of thermoelectric thermometry. Springer Science \& Business Media, 1998.

[16] F. Bucholtz and E. Udd, "Fiber optic magnetic sensors," pp. 369-382, 1991.

[17] H. Golnabi, "Design and operation of a fiber optic sensor for liquid level detection," Opt. Lasers Eng., vol. 41, no. 5, pp. 801-812, May 2004.

[18] B. P. Pal, Fundamentals of fibre optics in telecommunication and sensor systems. Bohem press, 1992.

[19] S. J. Mihailov, "Fiber bragg grating sensors for harsh environments," Sensors, vol. 12, no. 2, pp. 1898-1918, Feb. 2012.

[20] A. D. Kersey and T. A. Berkoff, "Fiber-optic bragg-grating differentialtemperature sensor," IEEE Photon. Technol. Lett., vol. 4, no. 10, pp. 1183-1185, Oct. 1992.

[21] Y. Zhao, P. Li, and Z. Pu, "MJ internal thread used for aerospace and its noncontact test method with a fiber optic sensor," Optical Engineering for Sensing and Nanotechnology, vol. 3740, pp. 501-504, May 1999.

[22] Y. Zhao, P. Li, and Z. Pu, "Shape measurement based on fiber-optic technique for complex internal surface," Measurement, vol. 30, no. 4, pp. 289-295, Dec. 2001.

[23] Y. Zhao, P. Li, C. Wang, and Z. Pu, "A novel fiber-optic sensor used for small internal curved surface measurement," Sensors and actuators A: Physical, vol. 86, no. 3, pp. 211-215, Nov. 2000. 
[24] R. Kashyap, "Photosensitive optical fibers: Devices and applications," Optical Fiber Technology, vol. 1, no. 1, pp. 17-34, 1994.

[25] N. Sabri, S. Aljunid, M. Salim, and S. Fouad, "Fiber optic sensors: short review and applications," pp. 299-311, Dec. 2015.

[26] B. Lee, "Review of the present status of optical fiber sensors," Optical fiber technology, vol. 9, no. 2, pp. 57-79, Apr. 2003.

[27] E. Udd, "Fiber optic smart structures," Fiber Optic Sensors and Introduction for Engineers and Scientists, Jul. 2011.

[28] K. Grattan and T. Sun, "Fiber optic sensor technology: an overview," Sensors and Actuators A: Physical, vol. 82, no. 1-3, pp. 40-61, May 2000.

[29] T. Wei, X. Lan, H. Xiao, Y. Han, and H.-L. Tsai, "Optical fiber sensors for high temperature harsh environment sensing," IEEE I2MC, pp. 1-4, May 2011.

[30] P. Dumon, W. Bogaerts, V. Wiaux, J. Wouters, S. Beckx, J. Van Campenhout, D. Taillaert, B. Luyssaert, P. Bienstman, D. Van Thourhout, and R. Baets, "Low-loss SOI photonic wires and ring resonators fabricated with deep UV lithography," IEEE Photon. Technol. Lett., vol. 16, no. 5, pp. 1328-1330, May 2004 .

[31] Q. Xu, B. Schmidt, S. Pradhan, and M. Lipson, "Micrometre-scale silicon electro-optic modulator," Nature, vol. 435, no. 7040, pp. 325-327, May 2005.

[32] A. W. Snyder and J. Love, Optical waveguide theory. Springer Science \& Business Media, 2012. 
[33] H. K. Bal, "Optical fibre refractive index, voltage and strain sensors: fabrication and applications," Ph.D. dissertation, Centre for Telecommunication and Microelectronics, Victoria University, Australia, 2011.

[34] J.-M. Liu, Photonic devices. Cambridge University Press, 2009.

[35] Z. He, P. Wang, and X. Ye, "Novel endoscopic optical diagnostic technologies in medical trial research: recent advancements and future prospects," BioMedical Engineering OnLine, vol. 20, no. 1, pp. 1-38, 2021.

[36] Z. Zou, Z. Qin, Y. Li, and Q. Liu, "Study on a health monitoring and defect imaging technology of special equipment that is based on optical fiber ultrasonic sensing," Optical Fiber Technology, vol. 61, p. 102393, 2021.

[37] X.-d. Wang and O. S. Wolfbeis, "Fiber-optic chemical sensors and biosensors (2013-2015)," Analytical chemistry, vol. 88, no. 1, pp. 203-227, 2016.

[38] X. Liu, T. Shang, D. Chen, and X. Wu, "A simple multifunctional broadband dispersion compensation and frequency conversion scheme by utilizing a polarization division multiplexing dual-parallel mach-zehnder modulator," Optics and Lasers in Engineering, vol. 137, p. 106332, 2021.

[39] H. Abdel-Gawad, "Solutions of the generalized transient stimulated raman scattering equation. optical pulses compression," Optik, vol. 230, p. 166314, 2021.

[40] J. A. Buck, Fundamentals of optical fibers. John Wiley \& Sons, 2004.

[41] D. B. Keck, "Optical fiber waveguides," Fundamentals of Optical Fiber Communications, pp. 1-107, 1981. 
[42] G. Lifante, T. Balaji, and A. Muñoz-Yagüe, "Planar optical waveguides fabricated by molecular beam epitaxy of pb-doped $\mathrm{CaF}_{2}$ layers," Appl. Phys. Lett., vol. 70, no. 16, pp. 2079-2081, Jun. 1997.

[43] R. Stoffer, H. Hoekstra, R. De Ridder, E. Van Groesen, and F. Van Beckum, "Numerical studies of 2D photonic crystals: Waveguides, coupling between waveguides and filters," Opt. Quantum Electron, vol. 32, no. 6-8, pp. 947-961, Aug. 2000.

[44] K. Okamoto, Fundamentals of optical waveguides. Academic press, 2006.

[45] D. Marcuse, Theory of dielectric optical waveguides. Elsevier, 2013.

[46] K. Safa, Optoelectronics and Photonics: Principles and Practices. Pearson Education India, 2009.

[47] S. O. Kasap, Principles of electronic materials and devices. Tata McGraw-Hill, 2006.

[48] W. L. Barnes, A. Dereux, and T. W. Ebbesen, "Surface plasmon subwavelength optics," Nature, vol. 424, no. 6950, pp. 824-830, Aug. 2003.

[49] D. K. Gramotnev and S. I. Bozhevolnyi, "Plasmonics beyond the diffraction limit," Nature photonics, vol. 4, no. 2, pp. 83-91, Jan. 2010.

[50] W. L. Barnes, "Surface plasmon-polariton length scales: a route to subwavelength optics," Journal of optics A: pure and applied optics, vol. 8, no. 4, p. S87, Jan. 2006. 
[51] R. W. Wood, "XLII. on a remarkable case of uneven distribution of light in a diffraction grating spectrum," The London, Edinburgh, and Dublin Philosophical Magazine and Journal of Science, vol. 4, no. 21, pp. 396-402, Apr. 1902.

[52] J. Zenneck, "Über die fortpflanzung ebener elektromagnetischer wellen längs einer ebenen leiterfläche und ihre beziehung zur drahtlosen telegraphie," Annalen der Physik, vol. 328, no. 10, pp. 846-866, 1907.

[53] H. Raether, Surface plasmons on smooth surfaces. Springer, Feb. 1988.

[54] S. A. Maier and H. A. Atwater, "Plasmonics: Localization and guiding of electromagnetic energy in metal/dielectric structures," J. Appl. Phys., vol. 98, no. 1, p. 10, Mar. 2005.

[55] M. Born and E. Wolf, "Basic properties of the electromagnetic field," Principles of optics, vol. 44, 1980.

[56] C. F. Bohren and D. R. Huffman, Absorption and scattering of light by small particles. John Wiley \& Sons, 2008.

[57] R. E. Hummel, Optische Eigenschaften von Metallen und Legierungen. Springer, 1971.

[58] P. Martyniuk, J. Antoszewski, M. Martyniuk, L. Faraone, and A. Rogalski, "New concepts in infrared photodetector designs," Applied Physics Reviews, vol. 1, no. 4, p. 041102, 2014.

[59] Z. Han and S. I. Bozhevolnyi, "Radiation guiding with surface plasmon polaritons," Reports on Progress in Physics, vol. 76, no. 1, p. 016402, Dec. 2012. 
[60] D. Thomson, A. Zilkie, J. E. Bowers, T. Komljenovic, G. T. Reed, L. Vivien, D. Marris-Morini, E. Cassan, L. Virot, J.-M. Fédéli et al., "Roadmap on silicon photonics," J. Opt., vol. 18, no. 7, p. 073003, 2016.

[61] J. V. G. Conejos, "Addressing fiber-to-chip coupling issues in silicon photonics," Ph.D. dissertation, Universitat Politècnica de València, Valencia, 2010.

[62] R. G. Hunsperger and J. R. Meyer-Arendt, "Integrated optics: theory and technology," Appl. Opt, vol. 31, no. 3, p. 298, 1992.

[63] J. Reed, "Light-matter interactions of plasmonic nanostructures," Ph.D. dissertation, College of Sciences, University of Central Florida, USA, 2013.

[64] J. Doylend and A. Knights, "Design and simulation of an integrated fiber-tochip coupler for silicon-on-insulator waveguides," IEEE J. Sel. Top. Quantum Electron, vol. 12, no. 6, pp. 1363-1370, Dec. 2006.

[65] V. R. Almeida, R. R. Panepucci, and M. Lipson, "Nanotaper for compact mode conversion," Opt. Lett., vol. 28, no. 15, pp. 1302-1304, 2003.

[66] T. Shoji, T. Tsuchizawa, T. Watanabe, K. Yamada, and H. Morita, "Low loss mode size converter from $0.3 \mu \mathrm{m}$ square si wire waveguides to singlemode fibres," Electron. Lett., vol. 38, no. 25, pp. 1669-1670, 2002.

[67] H. Subbaraman, X. Xu, A. Hosseini, X. Zhang, Y. Zhang, D. Kwong, and R. T. Chen, "Recent advances in silicon-based passive and active optical interconnects," Opt. Express, vol. 23, no. 3, pp. 2487-2511, 2015. 
[68] D. Van Thourhout, G. Roelkens, R. Baets, W. Bogaerts, J. Brouckaert, P. Debackere, P. Dumon, S. Scheerlinck, J. Schrauwen, D. Taillaert et al., "Coupling mechanisms for a heterogeneous silicon nanowire platform," Semicond. Sci. Technol, vol. 23, no. 6, p. 064004, 2008.

[69] S. Nambiar, P. Sethi, and S. K. Selvaraja, "Grating-assisted fiber to chip coupling for soi photonic circuits," Applied Sciences, vol. 8, no. 7, p. 1142, 2018.

[70] Y. Tang, Z. Wang, L. Wosinski, U. Westergren, and S. He, "Highly efficient nonuniform grating coupler for silicon-on-insulator nanophotonic circuits," Opt. Lett., vol. 35, no. 8, pp. 1290-1292, 2010.

[71] D. Taillaert, F. Van Laere, M. Ayre, W. Bogaerts, D. Van Thourhout, P. Bienstman, and R. Baets, "Grating couplers for coupling between optical fibers and nanophotonic waveguides," Japanese Journal of Applied Physics, vol. 45, no. 8R, p. 6071, 2006.

[72] A. Zakery and S. Elliott, "Optical properties and applications of chalcogenide glasses: a review," J. Non. Cryst. Solids, vol. 330, no. 1-3, pp. 1-12, Nov. 2003.

[73] K. Tanaka and K. Shimakawa, Amorphous chalcogenide semiconductors and related materials. Springer Science \& Business Media, 2011.

[74] R. Wang, A. V. Rode, S. J. Madden, C. Zha, R. Jarvis, and B. Luther-Davies, "Structural relaxation and optical properties in amorphous $\mathrm{Ge}_{33} \mathrm{As}_{12} \mathrm{Se}_{55}$ films," J. Non. Cryst. Solids, vol. 353, no. 8-10, pp. 950-952, Apr. 2007. 
[75] R. Wang, S. Madden, C. Zha, A. V. Rode, and B. Luther-Davies, "Annealing induced phase transformations in amorphous $\mathrm{As}_{2} \mathrm{~S}_{3}$ films," J. Appl. Phys., vol. 100, no. 6, p. 063524, Sept. 2006.

[76] G. W. Burr, M. J. Brightsky, A. Sebastian, H.-Y. Cheng, J.-Y. Wu, S. Kim, N. E. Sosa, N. Papandreou, H.-L. Lung, H. Pozidis et al., "Recent progress in phase-change memory technology," IEEE J. ETCAS, vol. 6, no. 2, pp. 146-162, Jun. 2016.

[77] P. Němec, M. Frumar, B. Frumarova, M. Jelınek, J. Lančok, and J. Jedelskỳ, "Pulsed laser deposition of pure and praseodymium-doped Ge-Ga-Se amorphous chalcogenide films," Optical Materials, vol. 15, no. 3, pp. 191-197, 2000.

[78] P. Němec, M. Frumar, J. Jedelskỳ, M. Jelınek, J. Lančok, and I. Gregora, "Thin amorphous chalcogenide films prepared by pulsed laser deposition," J. Non. Cryst. Solids, vol. 299, pp. 1013-1017, 2002.

[79] S. R. Ovshinsky, "Reversible electrical switching phenomena in disordered structures," Physical Review Letters, vol. 21, no. 20, p. 1450, 1968.

[80] A. Ahmadivand, B. Gerislioglu, R. Sinha, M. Karabiyik, and N. Pala, "Optical switching using transition from dipolar to charge transfer plasmon modes in ge 2 sb 2 te 5 bridged metallodielectric dimers," Scientific reports, vol. 7, no. 1, pp. 1-8, 2017.

[81] N. Yu, P. Genevet, M. A. Kats, F. Aieta, J.-P. Tetienne, F. Capasso, and Z. Gaburro, "Light propagation with phase discontinuities: generalized laws of reflection and refraction," science, vol. 334, no. 6054, pp. 333-337, 2011. 
[82] Z. Sámson, K. MacDonald, F. De Angelis, B. Gholipour, K. Knight, C. Huang, E. Di Fabrizio, D. Hewak, and N. Zheludev, "Metamaterial electro-optic switch of nanoscale thickness," Applied Physics Letters, vol. 96, no. 14, p. 143105, 2010.

[83] B. Gerislioglu, G. Bakan, R. Ahuja, J. Adam, Y. K. Mishra, and A. Ahmadivand, "The role of ge2sb2te5 in enhancing the performance of functional plasmonic devices," Materials Today Physics, vol. 12, p. 100178, 2020.

[84] B. Gholipour, "The promise of phase-change materials," Science, vol. 366, no. 6462, pp. 186-187, 2019.

[85] A.-K. U. Michel, P. Zalden, D. N. Chigrin, M. Wuttig, A. M. Lindenberg, and T. Taubner, "Reversible optical switching of infrared antenna resonances with ultrathin phase-change layers using femtosecond laser pulses," Acs Photonics, vol. 1, no. 9, pp. 833-839, 2014.

[86] J. Feldmann, M. Stegmaier, N. Gruhler, C. Ríos, H. Bhaskaran, C. Wright, and W. Pernice, "Calculating with light using a chip-scale all-optical abacus," Nature communications, vol. 8, no. 1, pp. 1-8, 2017.

[87] A.-A. Ahmed Simon, L. Jones, Y. Sakaguchi, H. Kunold, I. van Rooyen, and M. Mitkova, "Effect of ion irradiation on amorphous and crystalline Ge-Se and their application as phase change temperature sensor," Phys. Status Solidi B, p. 2000429 , Oct. 2020.

[88] A. Kolobov, P. Fons, and J. Tominaga, "Understanding phase-change memory alloys from a chemical perspective," Scientific reports, vol. 5, no. 1, pp. 1-11, 2015. 
[89] D. Adler, M. Shur, M. Silver, and S. Ovshinsky, Threshold Switching in Chalcogenide-Glass Thin Films. Springer, 1991.

[90] T. Kavetskyy, O. Shpotyuk, V. Balitska, G. Dovbeshko, I. Blonskyy, I. Kaban, W. Hoyer, M. Iovu, and A. Andriesh, "Vibrational and structural properties of unmodified and radiation-modified chalcogenide glasses for advanced optical applications," Sixth International Conference on Advanced Optical Materials and Devices, vol. 7142, p. 71420B, 2008.

[91] A. Barik, M. Bapna, D. Drabold, and K. Adarsh, "Ultrafast light induced unusually broad transient absorption in the sub-bandgap region of $\mathrm{GeSe}_{2}$ thin film," Scientific Reports, vol. 4, p. 3686, Jan. 2014.

[92] J. Woollam. Ellipsometry measurements. [Online]. Available: https://www. jawoollam.com/resources/ellipsometry-tutorial/ellipsometry-measurements

[93] A.-A. A. Simon, B. Badamchi, H. Subbaraman, Y. Sakaguchi, and M. Mitkova, "Phase change in Ge-Se chalcogenide glasses and its implications on optical temperature-sensing devices," J. Mater. Sci.: Mater Electron, May 2020.

[94] Y. Sakaguchi, D. Tenne, and M. Mitkova, "Structural development in Ge-rich Ge-S glasses," J. Non Cryst. Solids, vol. 355, no. 37-42, pp. 1792-1796, Oct. 2009 .

[95] C. Lin, H. Tao, Z. Wang, B. Wang, H. Zang, X. Zheng, and X. Zhao, "Defect configurations in Ge-S chalcogenide glasses studied by Raman scattering and positron annihilation technique," J. Non. Cryst. Solids, vol. 355, no. 7, pp. 438-440, Mar. 2009. 
[96] M. Mitkova, Y. Sakaguchi, D. Tenne, S. K. Bhagat, and T. L. Alford, "Structural details of Ge-rich and silver-doped chalcogenide glasses for nanoionic nonvolatile memory," physica status solidi (a), vol. 207, no. 3, pp. 621-626, Mar. 2010.

[97] X. Feng, W. Bresser, and P. Boolchand, "Direct evidence for stiffness threshold in chalcogenide glasses," Physical review letters, vol. 78, no. 23, p. 4422, Jun. 1997.

[98] B. Badamchi, A.-A. Ahmed Simon, M. Mitkova, and H. Subbaraman, "Chalcogenide Glass-Capped Fiber-Optic Sensor for Real-Time Temperature Monitoring in Extreme Environments," Sensors, vol. 21, no. 1616, Feb. 2021.

[99] S. Girard, J. Kuhnhenn, A. Gusarov, B. Brichard, M. Van Uffelen, Y. Ouerdane, A. Boukenter, and C. Marcandella, "Radiation effects on silica-based optical fibers: Recent advances and future challenges," IEEE Trans. Nucl. Sci., vol. 60, no. 3, pp. 2015-2036, Feb. 2013.

[100] Fiberguide Industries, "Buffer removal techniques for optical fiber," https:// www.fiberguide.com/supplier-resources/application-notes/, 2008, [Online; accessed Sept. 17, 2020].

[101] C. Brinker, G. Frye, A. Hurd, and C. Ashley, "Fundamentals of sol-gel dip coating," Thin solid films, vol. 201, no. 1, pp. 97-108, 1991.

[102] H. Schroeder, "Oxide layers deposited from organic solutions," Physics of thin films, vol. 5, pp. 87-141, 1969. 
[103] M. Mennig, P. W. d. Oliveira, A. Frantzen, and H. Schmidt, "Multilayer NIR reflective coatings on transparent plastic substrates from photopolymerizable nanoparticulate sols," Thin Solid Films, vol. 351, no. 1-2, pp. 225-229, 1999.

[104] A.-A. A. Simon, S. M. Rahmot Ullah, B. Badamchi, H. Subbaraman, and M. Mitkova, "Materials characterization of thin films printed with $\mathrm{Ge}_{20} \mathrm{Se}_{80}$ ink," Microscopy and Microanalysis, vol. 25, no. S2, pp. 2606-2607, Aug. 2019.

[105] A.-A. A. Simon, K. Kadrager, B. Badamchi, H. Subbaraman, and M. Mitkova, "Temperature sensing in nuclear facilities: Application of the phase change effect of chalcogenide glasses," NPICHMIT, Feb. 2019.

[106] J. Orava, T. Kohoutek, and T. Wagner, Deposition techniques for chalcogenide thin films. Elsevier, 2014.

[107] Y. Sakaguchi, T. Hanashima, K. Ohara, A.-A. A. Simon, and M. Mitkova, "Structural transformation in $\mathrm{Ge}_{x} \mathrm{~S}_{100-x}(10 \leq \mathrm{x} \leq 40)$ network glasses: Structural varieties in short-range, medium-range, and nanoscopic scale," Physical Review Materials, vol. 3, no. 3, p. 035601, Oct. 2019.

[108] J. Málek and J. Klikorka, "Crystallization kinetics of glassy $\mathrm{GeS}_{2}$," Journal of thermal analysis, vol. 32, no. 6, pp. 1883-1893, Nov. 1987.

[109] G. Dittmar and H. Schäfer, "Die kristallstruktur von germaniumdiselenid," Acta Crystallographica Section B: Structural Crystallography and Crystal Chemistry, vol. 32, no. 9, pp. 2726-2728, Mar. 1976. 
[110] S. Fouad, "On the glass transition temperature and related parameters in the glassy ge $\mathrm{Se}_{1-x}$ system," Physica B: Condensed Matter, vol. 293, no. 3-4, pp. 276-282, Jan. 2001.

[111] K. Inoue, O. Matsuda, and K. Murase, "Raman spectra of tetrahedral vibrations in crystalline germanium dichalcogenides, ges $_{2}$ and gese $_{2}$, in high and low temperature forms," Solid State Commun., vol. 79, no. 11, pp. 905-910, Sept. 1991.

[112] R. Eymard and A. Otto, "Optical and electron-energy-loss spectroscopy of GeS, GeSe, SnS, and SnSe single crystals," Phys. Rev. B, vol. 16, no. 4, p. 1616, Aug. 1977.

[113] G. Saffarini, "The effect of compositional variations on the glass-transition and crystallisation temperatures in Ge-Se-in glasses," Appl. Phys. A, vol. 74, no. 2, pp. 283-285, Feb. 2002.

[114] A. Morana, S. Girard, E. Marin, J. Périsse, J. Genot, J. Kuhnhenn, J. Grelin, L. Hutter, G. Mélin, L. Lablonde et al., "Radiation-hardened fiber bragg grating based sensors for harsh environments," IEEE Transactions on Nuclear Science, vol. 64, no. 1, pp. 68-73, 2016.

[115] A. Morana, S. Girard, E. Marin, C. Marcandella, P. Paillet, J. Périsse, J.-R. Macé, A. Boukenter, M. Cannas, and Y. Ouerdane, "Radiation tolerant fiber bragg gratings for high temperature monitoring at mgy dose levels," Optics letters, vol. 39, no. 18, pp. 5313-5316, 2014. 
[116] M. Wang, K. Zhao, J. Wu, Y. Li, Y. Yang, S. Huang, J. Zhao, T. Tweedle, D. Carpenter, G. Zheng et al., "Femtosecond laser fabrication of nanogratingbased distributed fiber sensors for extreme environmental applications," International Journal of Extreme Manufacturing, vol. 3, no. 2, p. 025401, 2021.

[117] Y. Li, M. Yang, D. Wang, J. Lu, T. Sun, and K. T. Grattan, "Fiber bragg gratings with enhanced thermal stability by residual stress relaxation," Optics express, vol. 17, no. 22, pp. 19 785-19 790, 2009.

[118] Z. Wang, F. Shen, L. Song, X. Wang, and A. Wang, "Multiplexed fiber fabryperot interferometer sensors based on ultrashort bragg gratings," IEEE Photonics Technology Letters, vol. 19, no. 8, pp. 622-624, 2007.

[119] A. Leal-Junior, A. Frizera-Neto, C. Marques, and M. J. Pontes, "A polymer optical fiber temperature sensor based on material features," Sensors, vol. 18, no. 1, p. 301, 2018.

[120] S.-Y. Lee, "Design of a plasmonic switch using ultrathin chalcogenide phasechange material," Current Optics and Photonics, vol. 1, no. 3, pp. 239-246, 2017.

[121] M. Hatzakis, "Electron resists for microcircuit and mask production," J. Electrochem. Soc., vol. 116, no. 7, p. 1033, 1969.

[122] H. Pfeiffer, T. Groves, and T. Newman, "High-throughput, high-resolution electron-beam lithography," IBM J. Res. Dev., vol. 32, no. 4, pp. 494-501, 1988. 
[123] S.-L. Shy, J. Y. Yew, K. Nakamura, and C.-Y. Chang, "Application of directwrite electron-beam lithography for deep-submicron fabrication," 16th Annual BACUS Symposium on Photomask Technology and Management, vol. 2884, pp. 334-343, 1996.

[124] Z. Wang, D. Dai, Y. Shi, G. Somesfalean, P. H. Thylen, S. He, and L. Wosinski, "Experimental realization of a low-loss nano-scale Si hybrid plasmonic waveguide," Optical Fiber Communication Conference, p. JThA017, 2011.

[125] Y. Ruan, M.-K. Kim, Y.-H. Lee, B. Luther-Davies, and A. Rode, "Fabrication of high-Q chalcogenide photonic crystal resonators by e-beam lithography," Appl. Phys. Lett., vol. 90, no. 7, p. 071102, 2007.

[126] Y. Matsubara, J. Taniguchi, and I. Miyamoto, "Fabrication of three-dimensional hydrogen silsesquioxane resist structure using electron beam lithography," Japanese journal of applied physics, vol. 45, no. 6S, p. 5538, 2006.

[127] G. A. DeRose, L. Zhu, J. K. Poon, A. Yariv, and A. Scherer, "Periodic subwavelength electron beam lithography defined photonic crystals for mode control in semiconductor lasers," Microelectronic engineering, vol. 85, no. 5-6, pp. $758-760,2008$.

[128] D. L. Lee, Electromagnetic principles of integrated optics. Wiley, 1986.

[129] B. Rahman, D. Leung, S. Obayya, and K. Grattan, "Numerical analysis of bent waveguides: bending loss, transmission loss, mode coupling, and polarization coupling," Applied optics, vol. 47, no. 16, pp. 2961-2970, 2008. 
[130] B. Snyder and P. O'Brien, "Packaging process for grating-coupled silicon photonic waveguides using angle-polished fibers," IEEE Transactions on Components, Packaging and Manufacturing Technology, vol. 3, no. 6, pp. 954-959, 2013.

[131] N. Jiang, L. Ma, P. Xu, H. Yang, and Y. Hu, "An integrated optic polarized bragg grating based on commercial standard fiber using side-polishing and metal films coating technique," Optik, vol. 124, no. 20, pp. 4607-4609, 2013.

[132] K. T. Kim, D. H. Kim, and B. H. Lee, "Two dimensional displacement sensor using fiber bragg gratings embedded in a cylindrical metal cantilever," Sensor Letters, vol. 14, no. 12, pp. 1227-1230, 2016.

[133] C. Miguel Giraldo, J. Zúñiga Sagredo, J. Sánchez Gómez, and P. Corredera, "Demonstration and methodology of structural monitoring of stringer runs out composite areas by embedded optical fiber sensors and connectors integrated during production in a composite plant," Sensors, vol. 17, no. 7, p. 1683, 2017.

[134] C. Y. Kong and R. Soar, "Method for embedding optical fibers in an aluminum matrix by ultrasonic consolidation," Applied optics, vol. 44, no. 30, pp. 6325$6333,2005$.

[135] Y. Li, W. Liu, Y. Feng, and H. Zhang, "Ultrasonic embedding of nickel-coated fiber bragg grating in aluminum and associated sensing characteristics," Optical Fiber Technology, vol. 18, no. 1, pp. 7-13, 2012. 
[136] T. Monaghan, A. Capel, S. Christie, R. Harris, and R. Friel, "Solid-state additive manufacturing for metallized optical fiber integration," Composites Part A: Applied Science and Manufacturing, vol. 76, pp. 181-193, 2015.

[137] J. J. Schomer and M. J. Dapino, "High temperature characterization of fiber bragg grating sensors embedded into metallic structures through ultrasonic additive manufacturing," 2017.

[138] C. M. Petrie, N. Sridharan, M. Subramanian, A. Hehr, M. Norfolk, and J. Sheridan, "Embedded metallized optical fibers for high temperature applications," Smart Materials and Structures, vol. 28, no. 5, p. 055012, 2019.

[139] C. M. Petrie, "Embedding sensors in metal and ceramic structures," Oak Ridge National Lab.(ORNL), Oak Ridge, TN (United States), Tech. Rep., 2020.

[140] A. Ghazanfari, W. Li, M. C. Leu, Y. Zhuang, and J. Huang, "Advanced ceramic components with embedded sapphire optical fiber sensors for high temperature applications," Materials \& Design, vol. 112, pp. 197-206, 2016. 


\section{APPENDIX A: \\ LIMITATIONS OF OUR EBL \\ TOOL-MODIFICATIONS PERFORMED TO \\ IMPROVE PERFORMANCE}


Before starting the fabrication process, we need to calibrate the EBL instrument in order to set the absolute scaling of Nanometer Pattern Generation System (NPGS) to $\pm 1 \%$ of the absolute scaling of the microscope. In the next step, it is normally sufficient to check for typical problems of the beam blanker. With our tool, We faced three problems: 1) beam blanker leakage, 2) astigmatism problem, and 3) low beam current.

\section{A.1 Beam blanker leakage}

The beam blanker deflects the beam off axis and protects the specimen from unnecessary exposure. Selecting the item again releases the blanker and returns the beam to scan the specimen. When the beam is blanked, the beam should not hit the sample. A simple test is put the beam into a Faraday cup on the sampler holder and check the beam current. Since our SEM did not have a fast blanker, the idle time during exposures caused extra exposure dots at the endpoints of every pattern. Also, during patterning, unwanted beam spots occurred on the pattern as shown in AFM images of Figure. A.1. 


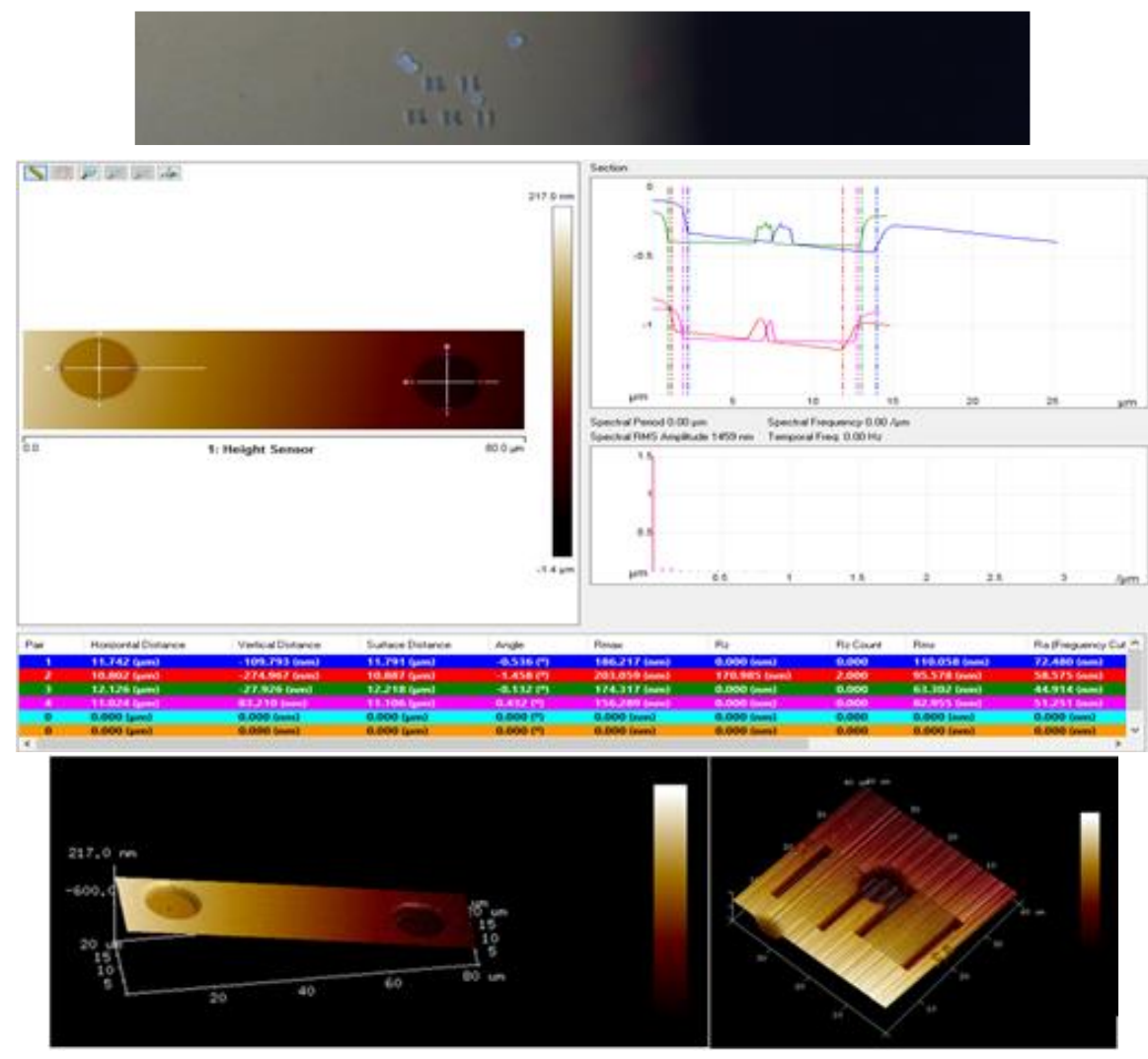

Figure A.1: Beam blanker causes extra beam spots during idle time of the exposure.

Per conversation with Boise State cleanroom staff and the e-beam tool manufacturer, we tried several options, including changing different settings to solve this problem. Figure. A.2 shows the settings we chose in NPGS software system files 


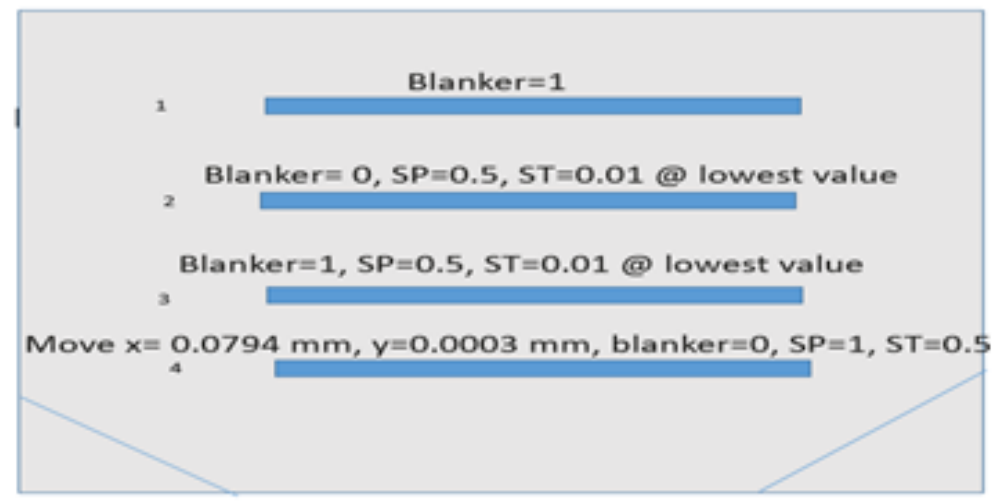

Figure A.2: Different beam blanker setting in system file.

SOI chips were patterned with these settings, and the images resulting from each of these settings is shown in Figure. A.3.

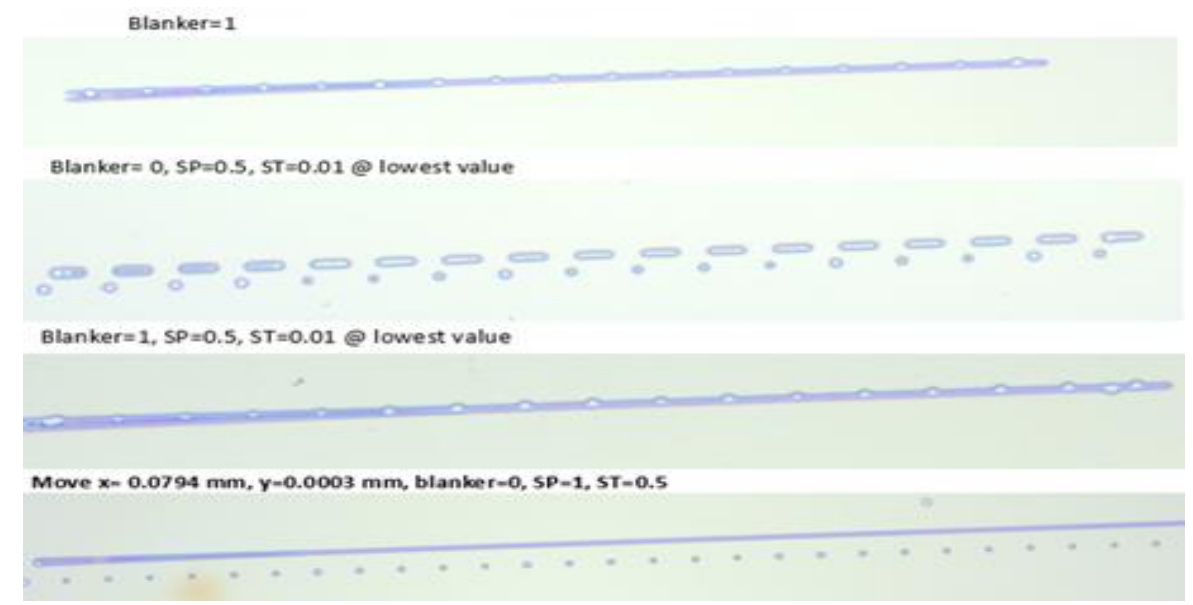

Figure A.3: Different beam blanker setting in system file.

It is shown that when the blanker is off (blanker $=0$ ), or blanker is on (blanker $=1$ ), there are beam spots on the pattern. The solution is to have a fixed dump location at 
the edge of the field of view to which the beam is moved whenever it normally would be blanked. We turned off the blanker (blanker $=0)$. The settle point $(\mathrm{SP})$ and settle To $(\mathrm{ST})$ are set to 1 and 0.5 , respectively. In the case, the blanker is off and the beam is positioned at a default dump location $(\mathrm{x}=0.0794 \mathrm{~mm}, \mathrm{y}=0.0003 \mathrm{~mm})$. At the final point in the pattern, the beam will be positioned at the dump point for current window. We selected this case for the rest of patterning. One issue is that even with this choice in beam blanker setting, there was still beam leakage at random points of the substrate or pattern. To solve this issue, support services completely changed the beam blanker. The fast beam blanker was installed in TENEO FEI instrument. The spots problem was fixed after this upgrade. Two designs were patterned to check the leakage of the beam blanker with different beam doses [ Figure. A.4(a)] and stitching method [Figure. A.4 (b)]. A microscope image of the patterned design confirms that there were no unwanted beam spots on the chip during the patterning. 


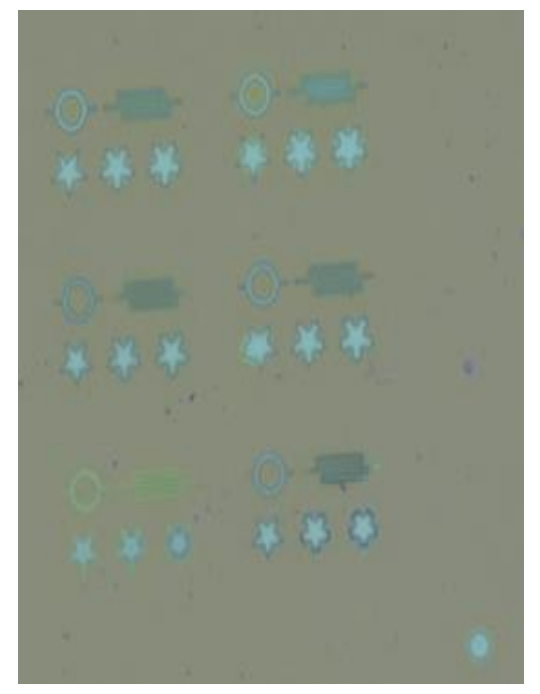

(a)
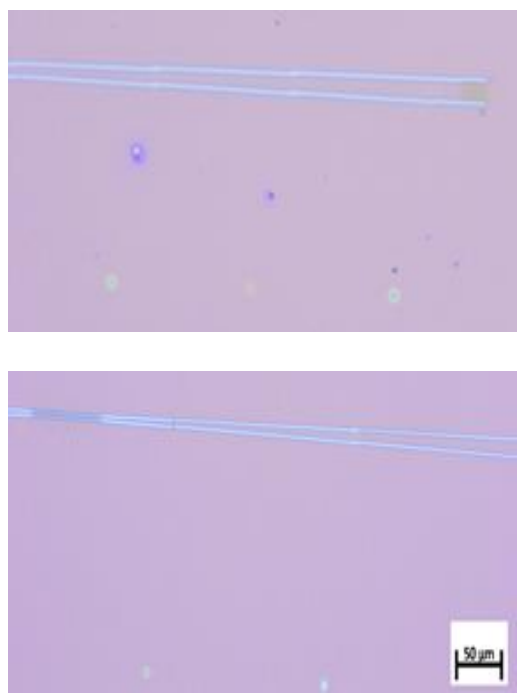

(b)

Figure A.4: Image of patterned designs after fixing beam blanker issue, a) pattern with different beam dose, b) stitching.

\begin{abstract}
A.2 Astigmatism problem
There was an issue with correcting astigmatism due to some heavy contamination/corrosion of the T1 and T2 detectors in the pole piece in FEI TENEO, as shown in Figure. A.5. We tried to clean the detectors and lower pole piece to no avail. It is required us to replace the entire lower column to correct the astigmatism. Existence of these contaminations will shift the beam and affect the fields in pole pieces. To solve these issues the brand new detector was installed and the contaminated one was cleaned.
\end{abstract}



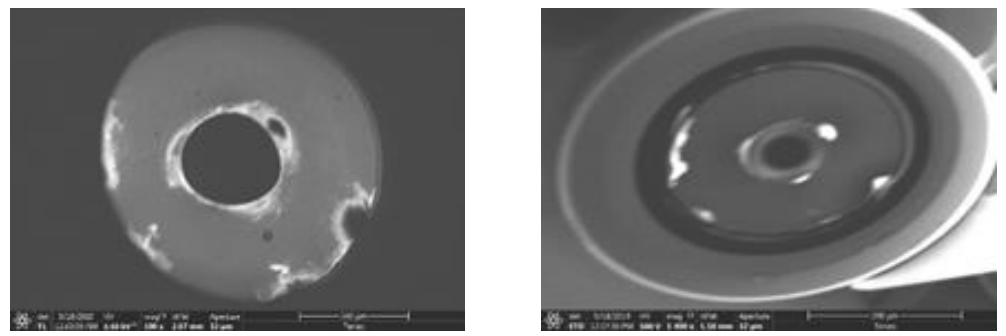

Figure A.5: Heavy contamination in T1 and T2 detectors.

\section{A.3 Low beam current}

We patterned the design with different line doses, and the atomic-force microscope (AFM) images are shown in Figure. A.6. After several tries, we observed that the beam current was different from the setting value in the SEM. To make sure that the problem was with the beam current and not with the Run file, we patterned one sample with area dose of $400 \mu \mathrm{C} / \mathrm{cm}^{2}$ and magnification of 500 from NPGS library, as shown in Figure. A.7. This pattern contained a $3 \times 2$ array of a variety of complex polygons. Each element was designed with a different color so that different dosages may be assigned. From these tests, we confirmed that problem was with the beam current. To test the beam current during patterning, we connected external beam current measurement to the SEM. Also, we made a Faraday cup to put into the chamber to catch the charged particles in vacuum and check the beam current. The beam current in external current measurement device should be equal to the value in the SEM. Rebooting the device solved the problem. To avoid such issues, we started using a Faraday cup during patterning to ensure proper beam current. 

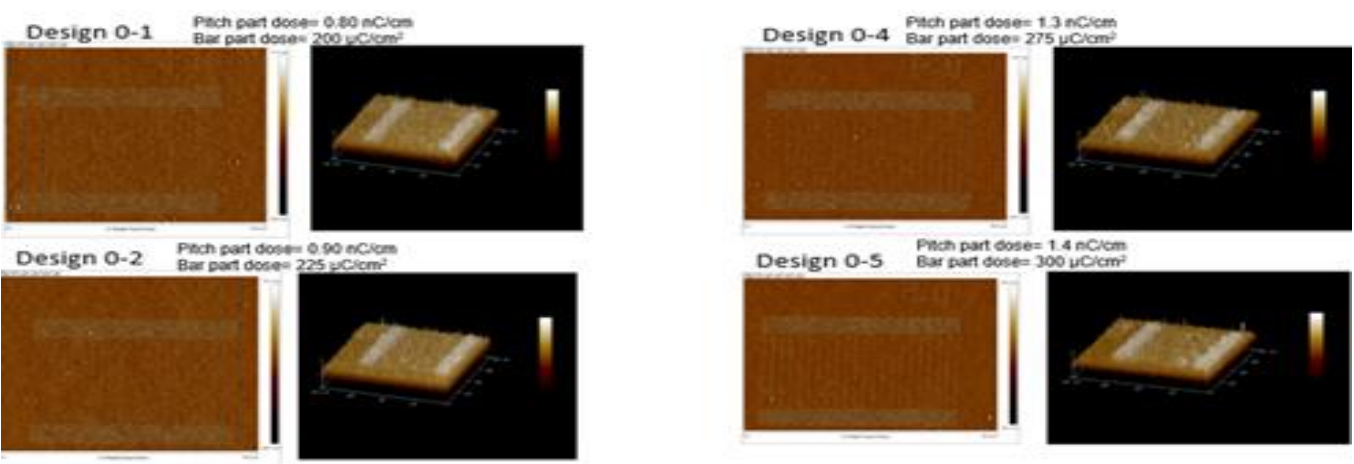

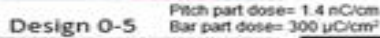

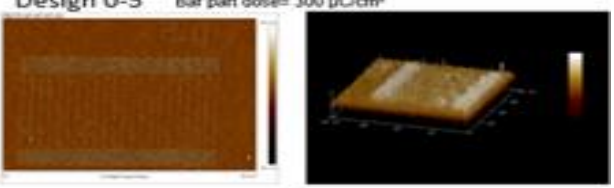

Design 0-3 Pach part dose $=1.2 \mathrm{nCK}$

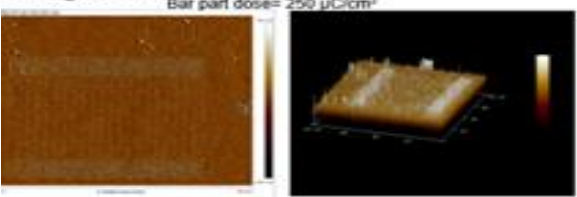

Figure A.6: Beam current issue during patterning.
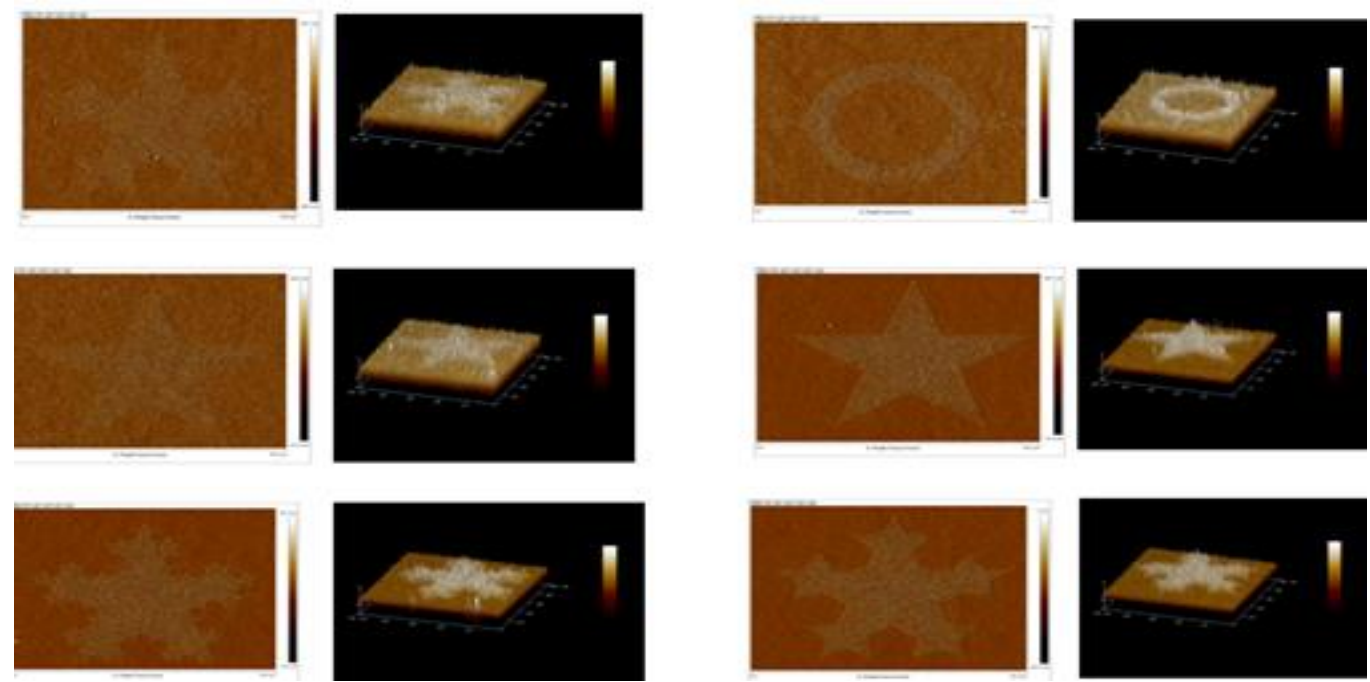

Figure A.7: Testing beam issue on design in NPGS library. 


\section{APPENDIX B:}

\section{FABRICATION PROCESS DETAILS}


Step 1. Substrate preparation: Substrate preparation is important for nanolithography in that a clean substrate is necessary for high-resolution patterning. Substrate contaminants can include some dirt particles or some organic or inorganic films. Disregarding this step could lead to a rough surface, poor reproduction of waveguide pattern, or decreased adhesion between resist and substrate, which would cause delamination of resist during the process. To carry out this step, we dip SOI samples in HF (1:10 diluted) for $10 \mathrm{sec}$ to remove native oxide. Next, we used acetone and isopropyl alcohol IPA to clean, blow dry under nitrogen gas, and store in clean and dry containers. The e-beam resist is coated immediately after this step to avoid creation of native oxide on the silicon surface. We have established through ellipsometer that application of resist immediately after wafer cleaning results in a surface roughness of $0.05 \mathrm{~nm}$, while the roughness prepared with normal process is about $0.5 \mathrm{~nm}$. In order to increase the adhesion of the photo-resist on the substrate and to remove the organic contamination, the SOI wafer is plasma ashing $\left(\mathrm{O}_{2}, \mathrm{CF}_{4}\right)$ with $300 \mathrm{~W}$ for 10 minutes. To eliminate any contaminant on the substrate, the wafer is placed on spin coater, and acetone and IPA are used to clean the substrate. Then, the wafer is placed on a hotplate for $75 \mathrm{~s}$ at $180{ }^{\circ} \mathrm{C}$ to remove any remaining water and solvents.

Step 2. Photoresist coating: On the cleaned substrate, a thin layer of ebeam is coated using spin coater. The thickness of the film can be controlled by controlling the spin speed, the time of coating, and e-beam concentration. We used PMMA photoresist in our fabrication process. Specifications of this resist are shown in Table B.1. The recipe for spin coating with 950 A5 PMMA is selected based on the curve in Figure. B.1 to achieve the required PMMA thickness. 
Table B.1: PMMA detail information

\begin{tabular}{cccccccc}
\hline Resist & Tone & Resolution & $\begin{array}{c}\text { Sensitivity } \\
\text { to EB }\end{array}$ & Contrast & $\begin{array}{c}\text { Film } \\
\text { life }\end{array}$ & $\begin{array}{c}\text { Sensitivity } \\
\text { to white light }\end{array}$ & $\begin{array}{c}\text { Etch } \\
\text { Resistance }\end{array}$ \\
\hline PMMA & Positive & $<5 \mathrm{~nm}$ & $\begin{array}{c}\text { Low, } \\
170 \mu \mathrm{C} / \mathrm{cm}^{2} \\
@ ~\end{array}$ & Low & Long & No & Poor \\
\hline
\end{tabular}

950PMMA A Resists

Solids: $2 \%-7 \%$ in Anisole

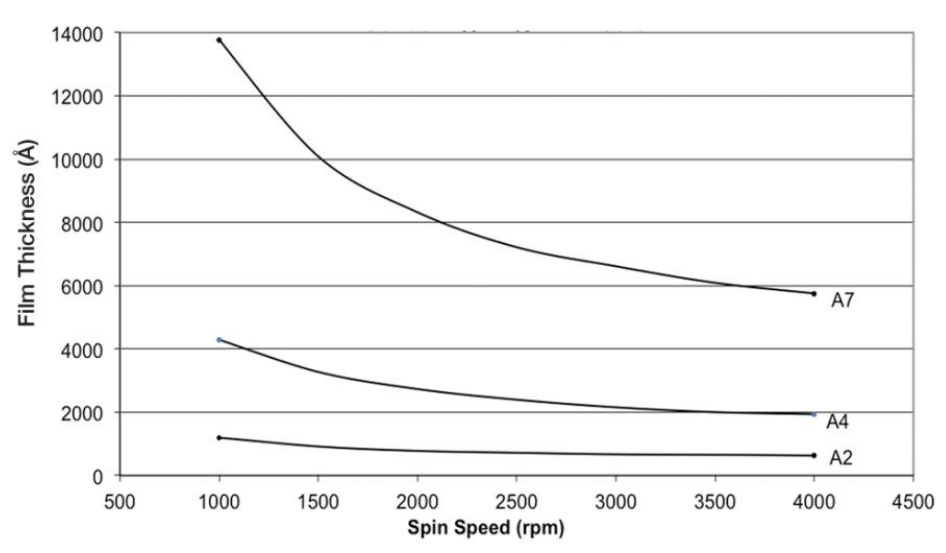

Figure B.1: The spin speed versus film thickness curves [http://microchem.com].

Our target thickness is about 250nm. The recipe is prepared in two steps for spin coating. In step 1 , Velocity $=500 \mathrm{RPM}$, Ramp $=500 \mathrm{RPM} / \mathrm{s}$, Time $=8 \mathrm{sec}$ and in step 2, Velocity $=4000 \mathrm{RPM}$, Ramp $=500 \mathrm{RPM} / \mathrm{s}$, Time $=90 \mathrm{sec}$. This recipe gives about 200-250 nm photoresist thickness.

In order to write high resolution pattern, we used ZEP520A positive photoresist. This EB resist shows high resolution, high sensitive and high dray etch resistance behaviors. For micron size features such as grating coupler pitches, using this resist is recommend. The ZEP520A with dilution rate of 1.7 is used to cover the wafer. We 
mixed $10 \mathrm{~g}$ of ZEP520A with $7 \mathrm{~g}$ of ZEP-A. The reason to dilute the photoresist is to obtain thinner thickness as shown in Fig. B.2. By adjusting the recipe in spin coater in two steps the thickness can reach about $280 \mathrm{~nm}$. In step 1, Velocity = $500 \mathrm{RPM}$, $\operatorname{Ramp}=500 \mathrm{RPM} / \mathrm{s}$, Time $=8 \mathrm{sec}$ and in step 2, Velocity $=1500 \mathrm{RPM}, \mathrm{Ramp}=500$ $\mathrm{RPM} / \mathrm{s}$, Time $=60 \mathrm{sec}$. This recipe gives about 200-250 nm photoresist thickness.

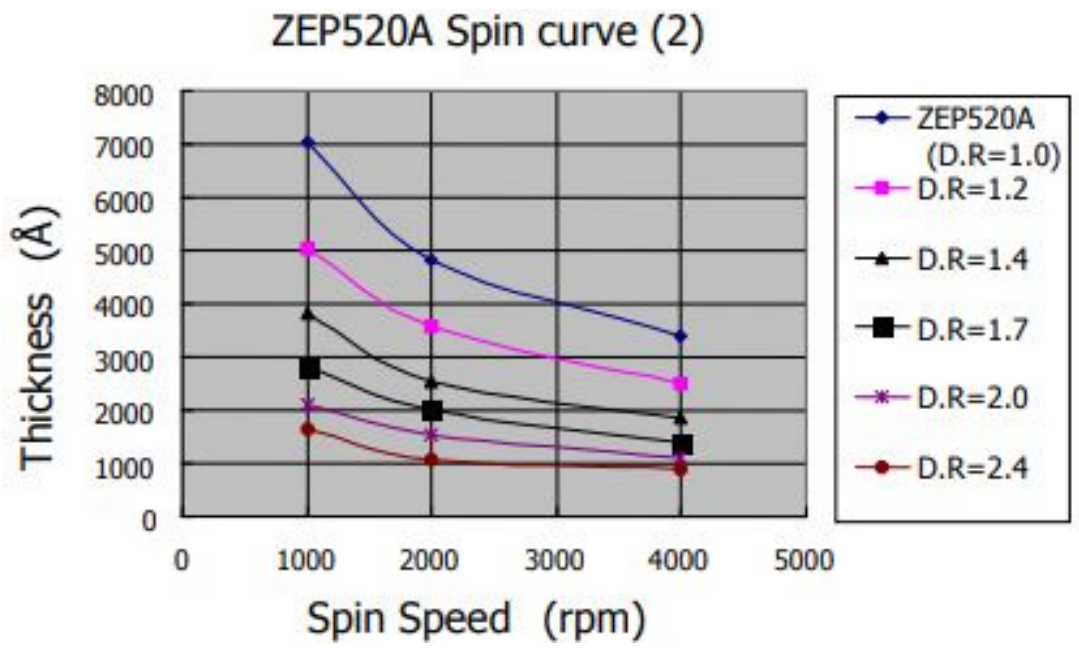

Figure B.2: The spin speed versus film thickness curves for ZEP 520A [ZEON corporation].

Step 3. Pre-exposure bake: After coating the photoresist layer on the wafer, the solvent needs to be removed. A pre-exposure bake or soft bake is used for removing extra solvent and for drying the photoresist after spin coating. The main purpose of this step is to decrease the amount of solvent in the film for achieving a stable film thickness. In our process, the sample is baked on a hot plate for 75 sec at $180{ }^{\circ} \mathrm{C}$.

Step 4. Photoresist thickness measurement: Ellipsometer is used to check the thickness of the photoresist after spin coating. To define the model in Ellipsometer, substrate is added as a layer with define material which you can find in the library of CompleteEase software. PMMA950 A5 is transparent layer. In ellipsometry 


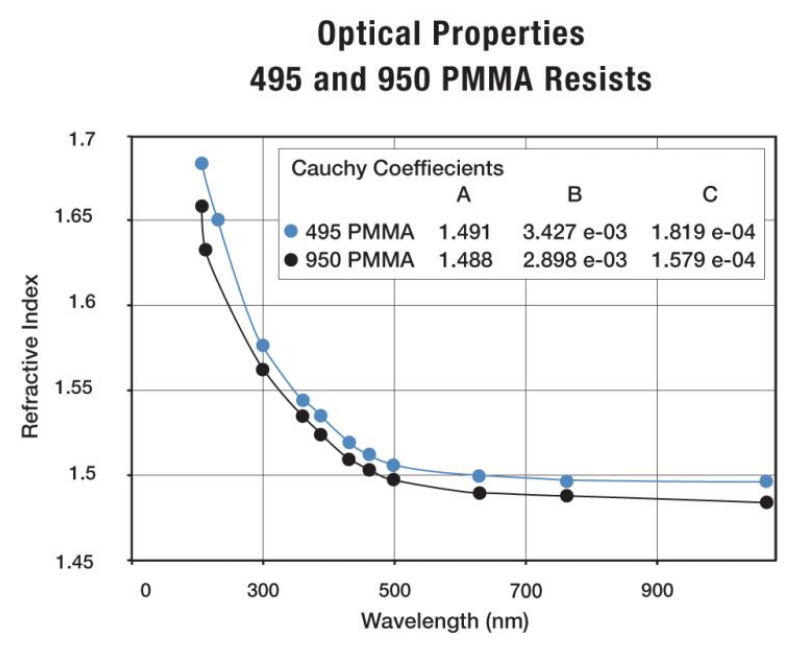

Figure B.3: The spin speed versus film thickness curves for ZEP 520A [ZEON corporation].

measurement Cauchy dispersion relation is used to model the index of refraction for transparent films. Cauchy coefficient of PMMA950 A5 as a top layer on substrate is selected from the curve from as shown in Fig. B.3. The thickness value is important for dose test in EBL and Reactive-Ion Etching (RIE). The same procedure is used to measure the thickness of the ZEP520A. In the ellipsometer model of ZEP520A layer on Si layer the Cauchy coefficient in the below equation is considered as:

$$
n=n_{0}+\frac{n_{1}}{\lambda^{2}}+\frac{n_{2}}{\lambda^{4}}
$$

Where $n_{0}=1.541093, n_{1}=4.113002 \times 10^{5}$, and $n_{2}=4.070357 \times 10^{12}$. Step 5- PostExposure Bake (PEB): This step is used for smoothening the photoresist. Exposing the sample to temperatures around $100-130{ }^{\circ} \mathrm{C}$ induces diffusion of the photoactive compound which smooths the standing wave ridges. 
Step 6. Exposure with EBL: After the completion of steps 1-5, the sample is now ready for e-beam lithography pattering. A TENEO FEI 2000 system within the Boise State Center for Material Characterization (BSCMC) is used for e-beam lithography. Grating coupler, taper lines, and waveguides were patterned using appropriate calibrated dose rates and energy. To pattern whole structure as the design length was larger than writing field of EBL, stitching method is decided to applied. Stitching involved writing multiple exposure field. The edge of each field accurately aligns with the nearest neighbor. The writing field axis must be aligned to the stage axis, otherwise, even with perfectly sized patterns written the correct distance apart, the field will be rotated and poor stitching will result. The EBL software can normally take care of fracturing the pattern in different writing field.

Step 7. Development: The development process has key role in controlling the fine features.Developers and development condition can strongly affect resolution and pattern quality due to the fact that contrast is a function of the developer. Developers are usually solvents that can selectively dissolve the exposed or unexposed resist materials. After exposure by EBL, the resist was developed. During development, $10 \mathrm{ml}$ of developer was maintained at room temperature. The samples were initially at room temperature (around $23{ }^{\circ} \mathrm{C}$ ). Development time was varied from $60 \mathrm{~s}$ to $90 \mathrm{~s}$ depending on the resist thickness, developer temperature and exposure dose. Isopropyl alcohol (IPA):methyl isobutyl ketone (MIBK) (3:1) for was chosen as a developer for PMMA photoresist because of its high contrast. The samples were then rinsed in IPA and DI water and carefully blow dried under nitrogen. This step is one of the most important steps of lithography. As PMMA is a positive photoresist, the exposed part is developed and removed by the developer, leaving the unexposed area 
as a mask for sequential pattern transfer, such as metal liftoff or reactive ion etching (RIE). The storage time between EBL exposure and development has never been considered to affect resolution in PMMA. Also, to develop ZEP520A the ZEP-N50 is used. Development without delay after lithography shows sub-10 $\mathrm{nm}$ feature sizes and high image contrast. Two weeks delay after lithography resulted in the disappearance of patterns less than $20 \mathrm{~nm}$. Such delay also resulted in poor image contrast and poor cross-sectional profile.

Step 8. Post Bake (Hard Bake): This step is used to harden the final resist to be stable in the harsh environments of etching. The temperature for this step is around $150{ }^{\circ} \mathrm{C}-200{ }^{\circ} \mathrm{C}$. This causes cross-linking within the polymer, which makes it more chemically stable.

Step 9. Initial characterization after exposure with EBL: To determine the variations in critical dimensions, we used Atomic Force Microscopy (AFM), optical profiler and a Zeiss microscope to examine the patterns. AFM analysis help us determine the optimal dosage for each section of the design. At these optimized dosages, all the channels are completely developed, sidewalls are vertical and smooth, and there are no resist residues at the bottom of the channel.

Step 10. Pattern Transfer: Reactive ion etching is a commonly used method to transfer patterns in the EBL resist into underlying layers, as illustrated in Fig. B.4. EBL is done on a resist-coated sample, and exposed resist is developed and removed by the developer. Using the remaining resist as a mask, the exposed substrate regions are etched. Finally, remaining resist is removed. Compared with wet etching, reactive ion etching offers less undercut and vertical sidewalls. Oxygen plasma is used to remove the remaining resist. We used Si Cryo-etch recipe (ICP 700W, RF 3W, -110 
${ }^{\circ} \mathrm{C}$ chunk temperature and 6 mTorr process pressure). Time in this recipe depends on photo-resist thickness. $\mathrm{SF}_{6}$ with 40 sccm flow, Helium is used as etchant gases.
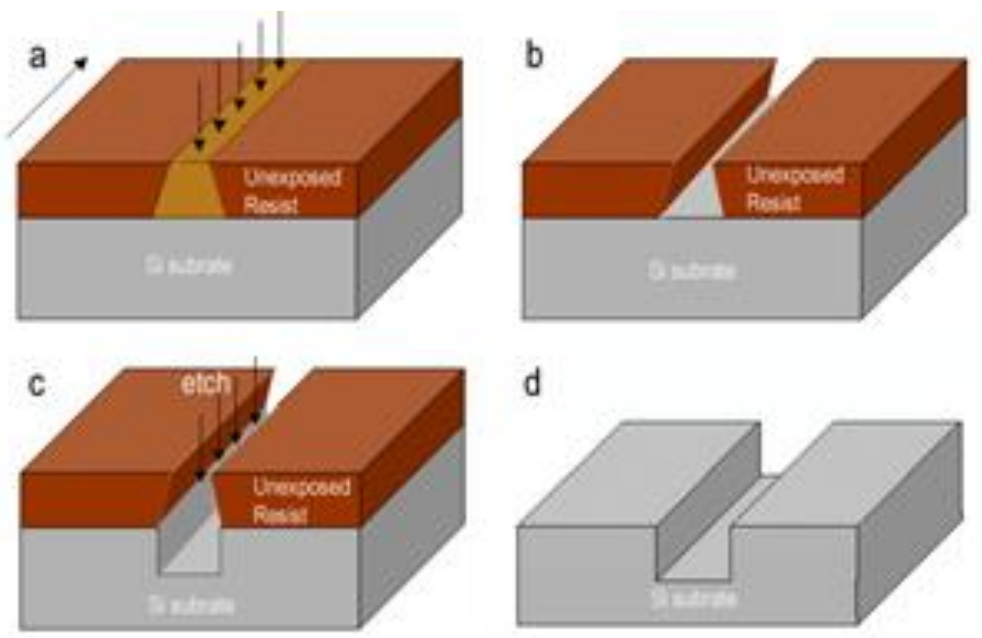

Figure B.4: Schematic of Reactive Ion Etching (RIE) after EBL. a) EBL is performed on the resist, b) exposed resist is developed, c) the substrate without resist covering is etched down by plasma, and $\mathrm{d}$ ) resist is removed. 